\title{
Semina selecta, e collectione anni 1851, quae hortus botanicus Imperialis Petropolitanus pro mutua commutatione offert.
}

Ceacia armata $\boldsymbol{R}$. Br.

dentifera Benth. pulchella $\boldsymbol{R}$. Br. verticillata $W$.

Acalypha brachystachya Hornem. caroliniana Walt.

Acanthocephalus amplexifolius Kar.,

Achyrachaena mollis Schauer.

Acinos graveolens Link.

Aconitum Gmelini Rchb. ochotense Rchb.

Actaea rubra Bigel.

Actinomeris helianthoides Nutt.

Actinospora dahurica Turcz.

Adenophora denticulata Fisch.

Adlumia cirrhosa Rafin.

Adonis caudata Stev.

squarrosa Stev.

Aegilops cylindrica Host. ovata $L$.

speltoides Tausch. sqnarrosa $\boldsymbol{L}$., Schreb. triaristata $\boldsymbol{W}$. triuncialis $L_{\text {. }}$, Willd ventricosa Tausch.

Agasyllis caucasica Spr.

Agrostis nitens Guss. pallida $D C$. retrofracta in

Ainsworthia cordala Boiss

Albuca fastigiata bryand.

Alehemilla pubescens $M$. $B$.

Allionia cucullata Fisch., Mey.

Allinm hymenorhizum Ledt.

- paradoxum Fisch.

Alonsoa caulialata Rivis., Pab.

Alopecurus utriculatus Pers.

Alsine globulosa C. A. Mey.

- laricifolia Wahlb.

- rubella Wahlb.

Alternauthera polygonoides $\boldsymbol{R} . \mathrm{Br}$.

- sesssilis $\boldsymbol{R}$. $\boldsymbol{B r}$. $\beta$ brachiata.

Alyssum confertum H. Berol.

- micranthum C. A. Mey.

- Szovitsianum Fisch., Mey. umbellatum Desv.

Amblyogyna polygonoitles Rafin.

Imellus annuus $W$.

- tridactylús DC.

Amethystea coerulea $\boldsymbol{L}$.

Ammobium alatum $R$. Bis.

Amsinckia angustifolia $\mathrm{Lehm}$.

- intermedia Fisch. Mey.

- Tycopsioides Lehm.

Anchnsa stylosa Stev.

Ancistiocarpus maypurensis ritumb. (ain Bonpl.

Androsace lactiflora fisch grandia. Andryala cheiranthifolia Hert. - integrifolia $t$.

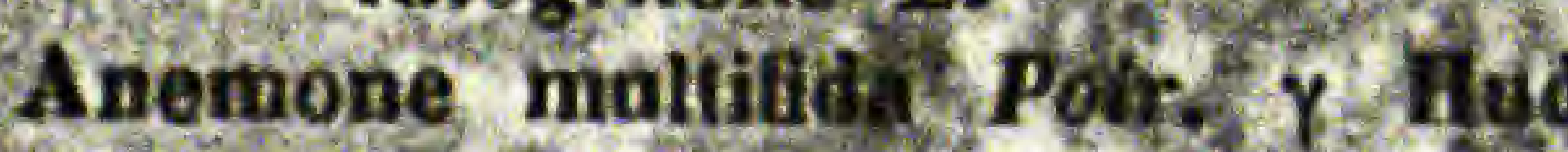

7 sonian $D C$. parcissinora $\boldsymbol{L}$.

Angelica anomala Lail

Angiab́tbas filifolius c, A. Mey.

Anisololus anthylloides Bernh:

- Wrangellianus Berit.

A poda cristata Schlechid.
Anoda liastata Cav.

Anomatheca juncea Ker.

Anthenis aurea $D C$.

- chia $L$.

- chrysantha Gay.

(chrysoleuca).

- melampodioides Hortor.

- nobilis $L$.

- relusa Del.

- rigescens $w$.

- ruthenica $M B B$.

Authriscus nemorosa $\mathbf{M B}$. tenerrima Boiss. torquata Duby.

Anychia dichotoma Mich.

Aquilegia advena Fisch., Mey

- atropurpurea $\boldsymbol{W}$.

- elata Ledb.

- Iragrans Benth.

- glandulosa Fisch.

- pubiflora Lindl.

- sibirica Lam.

Arabis Eschscholtziana Andrz.

- lilacina schrad.

- ludoviciana Fisch., Mey.

- pendula $\boldsymbol{L}$.

- stenopetala $w$. altaica.

Aralia racemusa $\boldsymbol{L}$.

Arbutus Metuziesii Pursh. $\beta$ DC.

Areinaria emarginata Brot. norvegica Gunn.

spathulata Desf.

Argemone albiflora Hornem.

- mexicana $\boldsymbol{L}$.

- ochroleuca Siceet.

Arnica montana $L$.

Artemisia macrobotrys Ledb.

Asclepias curassavica $\boldsymbol{L}$.

Asparagus caspius $H$. Vindob.

Aster sibiricus $\boldsymbol{L}$.

Astragalus contortuplicatus $\boldsymbol{L}$.

- juvenalis Del.

- prosiralus Fisch.

- sulcatus $\boldsymbol{L}$.

Astrantia Biebersteinii Trautv.

- helleborifolia Salisb.

Axyris amarantoides $L$.

- Lybrida $\boldsymbol{L}$.

Bahia arachnoidea Fisch., Lall.

Bambnsa spinosa Roxb.

Barkhausia Marschallii C. A. Mey.

Begonia Dregei Dietr., Otto.

- Porteriana Fisch, Mey,

- sinuata E. Mey.

Bellis ntegrifolia Mich.

Betckea major Fisch., Mey. samolifolia $D C$.

Betonica grandiflora $W$.

Bifora radians $M B$. testiculata $\mathrm{Spr}$.

Bignonia capreolata $\boldsymbol{L}$.

Blainvillea latifolia $\boldsymbol{D C}$.

Bosstaea linophylla $R$. Br. var.

- paucifolia Benth.

Bothriospermum teisellum Fisch,

Botryanthüs micranthus $\boldsymbol{H}$. Petr. Szóvitsianus C. A. Mey.

Bowlesia tenera Spr.

Boykinia aconitifolia Nutt.

Brachyconí iberidifolia Benth.

- spicatall $\boldsymbol{L}$.

- thyrsoidea $\boldsymbol{L}$.
Brassica Tournefortii Gouan. $\beta$ DC. Bromus adoënsis Hochst.

- brizaeformis Fisch., Mey.

- confertus MB. spiculis minorib. glabris.

- - - pubescentibus.

- oxyodon Schrenk.

- Schraderi Iunth.

- vestitus Schrad.

Broteroa trinervata Pers.

Bulbine semibarbata $\mathrm{Spr}$.

Bulliarda trichotoma Eckl., Zeyh.

Cacalia hastata $\boldsymbol{L}$.

Calceolaria glutinosa Regel.

- pinnata $L$

Calendula gracilis $D C$.

Calepina Corvini Desv.

Calimeris platycephala Cass.

Callicephalus nitens $C$. A. Mey.

Calliglossa Douglasii Hook., Arn.

Calliopsis Atkinsoniana Hook.

- Drummondi Sweet.

Callistachys ovata Sims.

- retusa Lodd.

Calotis amplexifolia C. A. Mey.

Camelina microcarpa Andrz.

Campanula alliariaefolia $\boldsymbol{W}$.

_. Loefflingii Brot.

Canna coccinea Ait.

- commutata Bouché.

- humilis Bouché.

- lagunensis Lindl.

- limbata Rosc.

- patens Rose.

- Sellovii H. Berol.

- speciosa Bot. mag.

- tenuiflsra Bouché

Capnophyllum cinerascens Fisch.

Carduus seninudus $M B$.

Mey.

Carex caucasica Stev.

Carmichaelia australis $\boldsymbol{R}$. $\mathrm{Br}$.

Carrichtera Vellae $\boldsymbol{D C}$.

Caucalis daucoides $\boldsymbol{L}$.

- $\beta$ iberica

Fisch., Mey.

- muricata H. Heidelb.

Caylusia abyssinica Fisch., Mey.

Celsia orlentalis $\boldsymbol{L}$.

Cenia geminata fínze.

- pruinosa DC.

Centaurea involucrata Desf.

- Kartschiana Scop.

- leptacantha Fisch., Mey.

- macrocephala Mless.-Prisenk.

- tenuiramea Del.

Centranthus macrosiphon Boiss.

Cephalaria tatarica Róm., Schult.

Cephalophora aromatica Schrad.

Cerastium carmpanulatum Viv. chloraetolium Fisch, Meg. dahurieum Fisch

dichotomum $\boldsymbol{L}$.

microspérmum C. A. Mey. nemorale $M B$.

perfoliatum $\boldsymbol{L}$.

pilosum Ledb.
Cerastium tetrandrum $\mathrm{Sm}$. $\beta$ asturicum Fisch., Mfey.

Ceratocapnos umbrosa Durieu.

Ceralocephalus falcatus ptrs.

- orthoceras $D C$.

Chaerophyllum Prescollii $D C$.

Chaeturus fasciculatus link.

Chamaerops humilis $\boldsymbol{L}$.

Chardinia xeranthemoides Desf.

Charieis heterophylla Cass.

Chenopodium atistatum $\boldsymbol{L}$.

Chlamydophora tridentata Ehrenb.

Chlora serotina Koch.

Chorispora tenella DC.

Cineraria lobata Herit.

Cinna mexicana $\boldsymbol{P}$. de $\boldsymbol{B}$.

Cirsium arachnoideum $M$ ISB.

- dealbatum $\boldsymbol{M B}$.

- munitum $\boldsymbol{M B}$.

- obvallatum $M B$.

- serratuloides DC.

Cistus candidissimus Dun.

- creticus $\boldsymbol{L}$

- garganicus Ten.

- incanus $\boldsymbol{L}$

- $\quad-\beta$ angustifolius.

- monspeliensis $\boldsymbol{L}$.

- tauricus Presl.

- undulatus Dien.

- vaginatus Ait.

- villosus Iam.

Claytonia alsinotdes Sims. fl. albo.

Cleome pungens $\boldsymbol{w}$.

- speciosissima Deppe.

- violacea $\boldsymbol{L}$.

Cleonia lusitanica $\boldsymbol{L}$.

Clethra arborea Ait.

Cliffortia ilicifolia $\boldsymbol{L}$.

Clivia nobilis Lindl.

Clypeola microcarpa Hort.

Collinsia bicolor Benth.

- grandiflora Lindl.

- parviflora Lindl.

- sparsillora Fisch., Mey.

Conioselinum Fischeri Wimm., Grab.

- Univitlatum Turcz.

Conium chaerophylloides Eckl, Zeyh.

Conringia planisiliqua Fisch., Mey.

Convolvulus farinosus $\boldsymbol{L}$.

- Pseudo-siculus Cav.

- siculus $\boldsymbol{L}$.

- undulatus Cav.

Conyza chilensis Spr.

- Gouani W.

Coreopsis coronata Hook.

Corethrostylis bracteata Endl.

Corispermum Pallasii Stev.

Cornucopiae cucullatum $\boldsymbol{L}$.

Corrigiola telephiifolia Poir.

Corydalis clavieulata $\boldsymbol{D C}$.

Cosmanthus fimbriatus Nolte.

- viscidus Alph. DC.

Cosmes bipinnatus Cav.

Cotoneaster acuminata Lindl.

- microphylla Wall.

- rotundifolia Wall.

- tonentosa Lindl.

Cotula anthemoides $\boldsymbol{J}$.

- coronopifolia $\boldsymbol{L}$.

filifolia Thb. 


\section{the o thate ghata Di phistiti $23 k$, so ur D DC. \\ Coustats knolk Fisch, Me}

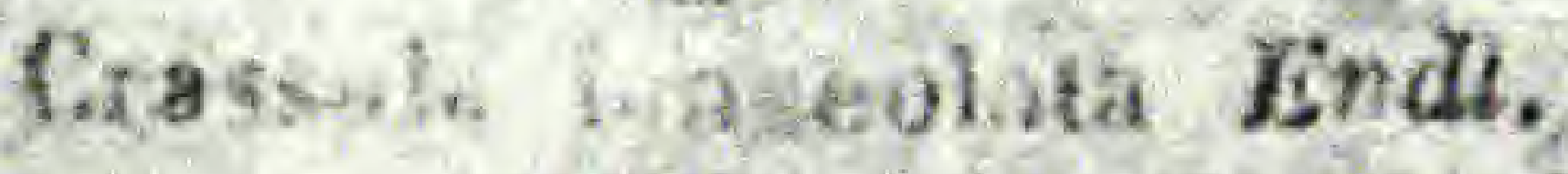
schimpari $C$. A. Hei.

Cremecephatum cernum Cass. Crepis multicaulis $x$ edb.

Gr talaria incana $\boldsymbol{L}$

Crucinaella angustifolia $L$.

- patula $\boldsymbol{L}$

Cryptantha glomerala Lelim.

- microcarpa Fisch, Mey.

Cryptocarya triplinervis $R$. Br.

Cuphea silenoides Nees.

Cycioloma platyphylla $\mathbf{M o q}$.

Cynoglossum glochidiatum Wall.

Cysticapnos africana Guertn.

Dactylaena nicrantha Solirad.

Dahlia Nerkii Lehm.

Datura laevis $\boldsymbol{L}$. fil.

- Tatula 1

Oaucus abyssinicus Fiseh., Mey

- glochidiatus Fisch.. Mey.

- montevidensis Link.

- pulcherrimus Koch.

Deeringia baceata Moq.

Delphinium dasyanthum Kar., Kïr.

- lasiostachyum Fisch., Mey.

Desmanthus virgatus $\boldsymbol{w}$.

Destauxia cornigera C. A. Mey.

Dianthus Cyri Fisch., Mey.

- Pseudo-Armeria IIB.

Dichelostemma congesta Krunth.

Dichrocephala latifolia $D C$.

- sonchifolia DC.

Din liptera resi pinata Iuss.

Diptes iridioides Stret

Dimetopia hirta lienth.

- isocarpa Dartl.

Diodia dasycephala Chom., Schlechid.

Dischisma captatun Chois.

- clandestinum E. Mey.

Dodecatheon integrifolium $\mathbf{~} \mathrm{Ki} /$.

Dolichos lignosus $\boldsymbol{L}$.

Dorycniom herbaceum rill

Draba borealis DC.

- casoliniana Walt.

Dracocephaium autans $\boldsymbol{L}$.

- parviforam Nutt. thymiflorum $L$.

Drummondia milelloides $D C$.

Echinaria capitata Desf.

Echinospermum affine Far., Kïr.

- cristatum Bong.

- laere C. A. Mey.

- patulum Lehm.

- Redowskii Lehm

- strictum Ledb.

Elcusine coracana Pers.

- oligostaebya Link.

Elscholtzia cristata $W$.

Elymus Capui-Medusae $L$

- crinitus Siltreb.

Emex spinosa Campd.

Enarthrocarpus tyratus $I C$.

Epilobium algidum $M B$.

- chilense H. Birol. minulum Lind

Erechtites carduifolia $D C$.

- hier aciifolia Rafin.

- valerianaefolia $D C$.

Eremogone longifolia Fen:l.

Frica baccans $\boldsymbol{L}$.

- urceolaris Berg.

Erigeron armerifolius Ture:.

- asperulus Fisch. Mey

- disiritu Wich.

ugatu- Ledb.
Frgater spathulatus Vest.

i chings 1

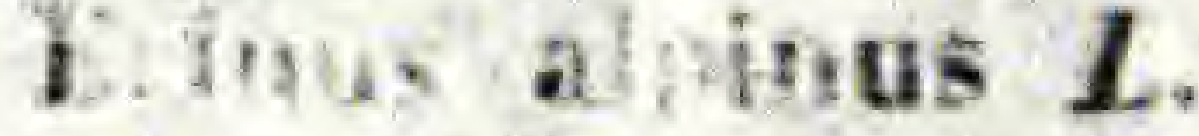

lirodiens atomarian Del.

- darquiniansm Fisch., Míy Lithreun Jeman.

maluehoides $W$.

Erpetion reniforine Sucet,

Erucastrum arabicum Fisch., aiey. Pollichii Schimp.

Eivarn chilense Fisch, Mey.

- Hohenackeri Fisch., Mey.

- Lilacinum Fisch., Mey.

- Loiseleurii $\mathbf{M B}$.

Erysimum Perofskianum Fisch. Mey.

- sisymbrioides C. A. Mey.

Eschscholtzia californica Cham.

Ethulia conyzoides $\boldsymbol{L}$.

- Kraussii C. H: Schultz.

Eucharidium conciunum Fisch., Mey.

- grandillorum Fisch, Mey.

Euclidium syriacum $\boldsymbol{R}$. $\boldsymbol{B r}$.

Euphorbia graeca Boiss.

- Lagascae Spr.

- Pithyusa $L$.

- prostrata Ait.

- pterococca Brot.

Eutoca Wrangelliana Fisch,, Mey

Evax asterisciflora Pers.

Fagopyrum emarginatum Maisn.

- Lataricum Gaertn.

Faldermannia parviflora Trautv.

Fedia graciliflora Fisch., .Mey.

Fritillaria pallidiflora Schrenk.

Fumaria agraria Lag.

- capreolata $L$. muralis Sonder. spicala $\boldsymbol{L}$.

Galactites Durieui Spach.

- mutabilis Dur.

- tomentosa Möncl.

Galega orientalis Lam.

Galinsogea brachystephana $\boldsymbol{H}$. Derol.

Galium caudatum Boiss.

- divaricatum Lam.

- intricatum Reut.

- rètrorsam $D C$

- tenuissimum $\boldsymbol{M B}$.

- verticillatum Danth.

Gamolepis Tagetes $D C$.

Garidella Nigellastrum $\boldsymbol{L}$.

Gastrolobium bilobum R. $\boldsymbol{B r}$. - spinosum Benth.

Gastrolychnis angustiflora Rupr.

Gatyona globulifera Cass.

Gaudinia fragilis $\boldsymbol{P}$. de $\boldsymbol{B}$.

Gaurs parriflora Hook

Genista canariens.s $\boldsymbol{L}$.

- chrysobotrys Fisch.

- Tadiala Scop.

Gentiana asclepiad $a$ a $L$.

- septemfida Fail.

Geranium affine Ledb.

- sibiricum $\boldsymbol{L}$

- Wlassorianum Fisch.

Gilia achilleifolia Benth. inconspicua Dougl. laciniata Ruiz., Pav.

- millefoliata Fiscin., Mly.

- tricolor Benth,

Glossocomia clematided Schrenk.

Gnafhalium cephatoidēun $\boldsymbol{W}$.

- pennsylvanicum $W$.

- resediefolium Trev.

- undulatum $L$.

Goudia lotifolia S Stisl.

firangea mider. s.atana Poir

Ciridulia maxicana fize.

Guirotia oleifera Df.

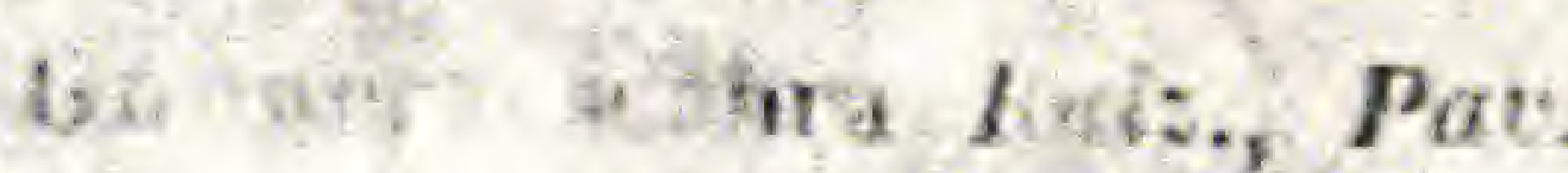
murieata Schrad.

trypsoptila degans $M E$.

scosi Hiurr.

Hardeabergia monophs Ha Benth.

ilebenstreitia cenuifolis Schrad.

Heden'na hispida Pursit.

Hedysarum elongatum Fiseh. neglectun, Ledl. spinosissimum $\boldsymbol{L}$.

Helenium brachyglossum Fisch.

Lall.

Nelianthemum Iasiocarpum H. Paris.

- tauricum Rupr.

- villosum Thib.

Heliophila amplexicaulis $\boldsymbol{L}$. fit.

- crithmifolia $w$.

- pendula $\boldsymbol{W}$.

- pilosa Lam. $\beta$ DC.

- pusilla $L$.

- stricta Sonder. angustifolia.

Ileiminthia echioides Gaertn.

Helosciadium crassipes Koch. $\beta$ heterophyllum

- laciniatum DC.

- leptopbyllum $D C$

Heracleum asperum $\boldsymbol{M B}$.

- barbatım Ledb.

- palnatifidum Fisch, Lall.

- persicum II. Paris.

- pubescens $.11 B$.

- villosum Hoffm.

- Wilhelnsii Fisch, Lall,

Herniaria odorata dndrz.

Heterospernum pinnatum Cav,

Heuchera americana $L$. cylindrica Dougl.

Hippocrepis ciliata $W$.

- unisiliquosa $\boldsymbol{L}$.

Hosackia Purshiana Benth.

Mymenocarpus numimularius $W$.

Hyoscyamus canariensis $\mathrm{Ker}$.

- orientalis $\mathbf{M B}$.

Hypericum Ascyron $L$.

- elegans Steph.

- grandifolium Chois.

- hyssopifolium Vill.

- linearifolium Vahl.

Iberis pectinata Boiss.

- virginica Fisch, Mey.

Jysanthes cubensis Fisch., Mey.

Impatiens parriflora $D C$,

- Roylei Walp.

Inula rhizocephala Schrent.

Iris Güldenstadtiana Lep.

- balophila Pall.

- ruthenica Ait.

- sibirica L. $\beta$ Laematophylla

Isatis orientalis $\mathrm{W}$. var. Fisch.

Isolę̧is Micheliana Röm., Schult.

- Saviana Setatt.

Jsopyrum funarioides $\boldsymbol{L}$.

Kalbfussia Mulleri schultz.

Kennedya rubicunda Vent.

Kentrophylium leucocaulon $D C$

Krubera leptophylla Moffin.

Krynitzhia leiocarpa Fisch., Mey.

Ktenospermum linifolium Lehm.

Lactuca altaica Fisch., Mey. cracuriensis Saviez.

Lagascea mollis Cav.

Lagoecia cuninoides $\boldsymbol{L}$.

Lagurus ovatus $\boldsymbol{L}$.

Lallemantia canescens Fisch., Mey.

- iberica Fisch, Mey

- peltata Fisch, Mey.

- Liosleana Benth.

Lamarchia aurea Mönch.

I muium bifotum Cyr.

1.5endith hispidar $M n$.

Latifoinm $\boldsymbol{L}$

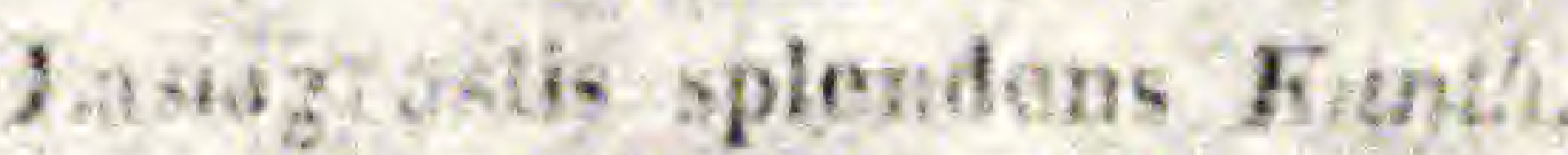

Batbyrus Iphaca $L$.

Cicera $\boldsymbol{L}$, angusticalin

- - - laufolias

- hirsutus $\boldsymbol{l}$.

- longegedan s!atus fowt

- Nissulia $\boldsymbol{L}$.

- OchrasDC.

- Pseudo-Aplaca Dofiss.

- sativus $\boldsymbol{L}$.

- sphaericus Retz.

- stans Vis.

Larandula inultifida $L$.

- pubescens Decais.

- Stoechas $L$.

Leonurus sibiricus $\boldsymbol{L}$.

Lepidium chilense $\boldsymbol{H}$. Paris.

- deusiflorum Schrad. Ecklonii Schrad.

- Menziesii DC.

- micranthum Ledb.

- perfoliatum $L$.

Lepidonema chilense Fisch, M

Lepigonum australe $F$. Mйll.

- canadense Fisck, Mey.

- glandulosum Fisch, Mey. Iaxifforum Barll.

Lentosiphon densifurus Benth.

Lepturus incursatus Frin.

Lepyrodiclis holosteoides Fisch Mey.

Lestibudesia virgata Röm., Schuit.

Leucanthemum pallevs $D C$.

Leucas martinicensis $\boldsymbol{R}$. $\boldsymbol{B r}$.

- Schimperi Hochst.

- urticaefolia $R$. Br.

Leucerla senecioides Hook, , for

Libanotis condensata Fisch, Hey.

Ligularia macrophilla $D C$.

- speciosa Fiseh, Mey.

Lilium teudifolium $F$ isch.

Limnanthes Dougtasii $\boldsymbol{R}, \boldsymbol{B}$.

Linaria athifrons spr.

- aparinoides Char.

- Coufidensis Dim.

- chalepensis Vill.

- cirrhosa 1.

- litoralis $I I$

$-\quad-3$ plerospern
If. Peimp.

- origanifolia $D C$. 


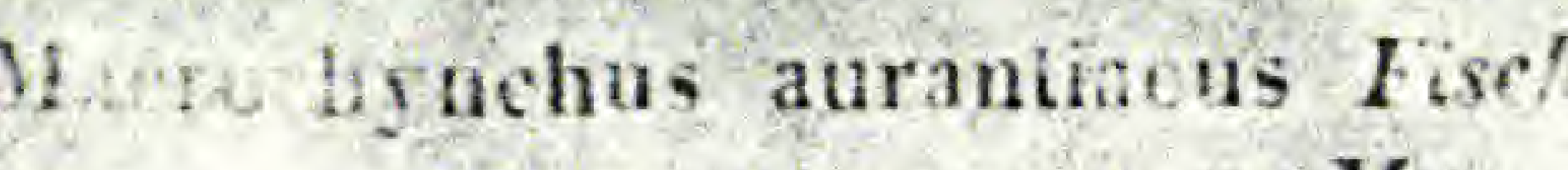

lacvigatus Fisch., Mey.

pterocarpus Fisch., Mey.

Midia elegans Don.

- mellosa Mrolin.

- racemosa Torr., Gray.

- stellata Fisch., Mey.

if thonia Aquifolium Nutt.

Malope grandifiora Hort. Angl.

Malva abyssinica Al. Braun.

- limensis $\boldsymbol{L}$.

- tricuspidata Ait.

Manulea rubra $T h b$.

Mariynia Iutea Lindl.

Maruta fuscata $D C$.

- Micheliana Fisch., Yey.

microcephala Schrenk.

Matricaria decipiens $\boldsymbol{C}$. d. Mey. discoidea $D E$.

nigellaefolia $D C$.

Yizu rugosus Lour.

Me is zo arborea $L$.

coronuta Lam.

deuticulala $W . \beta$ caspia.

disciformis $D C$.

ghulinosa.$I B$.

hetereocarpa Spach.

reticulata Benth.

secundiflora Dur.

striata Bast

M. Hompedism divaricatum $D C$.

- lougifolium Cav.

risomboideum $D C$.

Mohidrum bracbypetalum $F e n: l$.

Helica calfrorum Sctirad.

Slelilots Bonplandi Ten.

- graciiis $D C$

- messanensis Desf.

suareolans Ledb.

- Sulcata Desf.

Menton Requirmi Benth var. birtula.

'bacorialis violaefilia fomze.

Jiet bitera sobolifera Fisch, Wey.

Merabis - retnensis $D C$.

Hietsutheum linifulium Hort.

Microlonchus Clusii Spach.

elatus Spach.

tenelius Spach.

U to meria bonariensis Fisch, Mey. wata Benth.

Heropus californicus Fisch, Mey.

Mie atium fenaifolium Boiss.

Mitell diphylla $\boldsymbol{L}$.

Wodtota caroliniana Don.

Yobringas pentandira Gay.

Nolhor, set ishi Ser.

ularium $D C$.

mantica fintl.

gezaternella $L$, h.

Wormucais corrigi toides Fensl.

Himut ois trilila Setrant.

Honchy, is califormica Fisch., Mey.

labrata Fisete, Mm

D. jor $D C$.

lithiouar Fieh, Mey.

H)

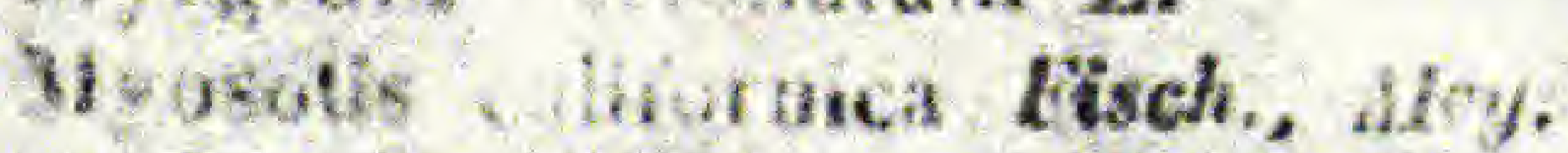

Mandidica perpugilla DC.

Waelethom thovirens $D C$.

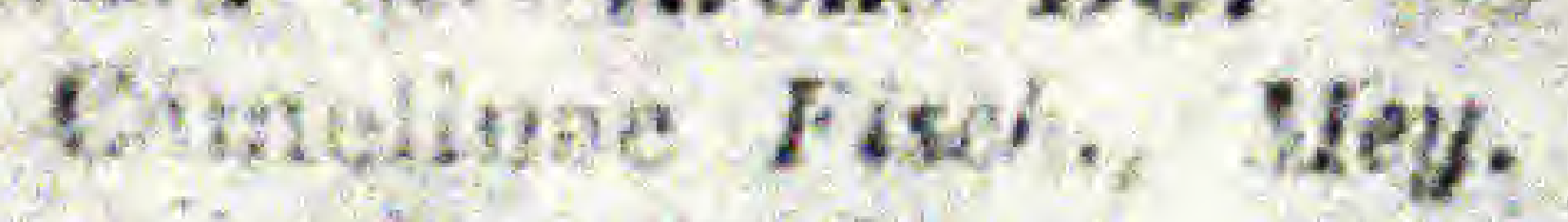

erreltim Treo

pist!'s II. Puris.

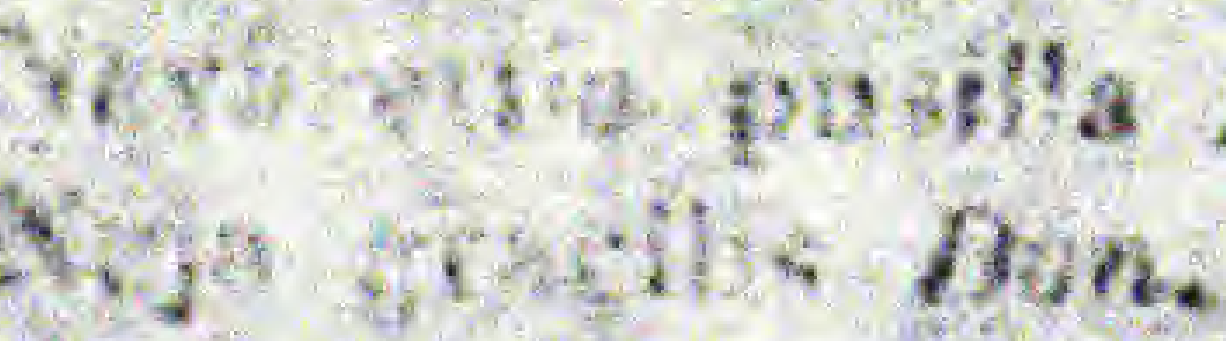

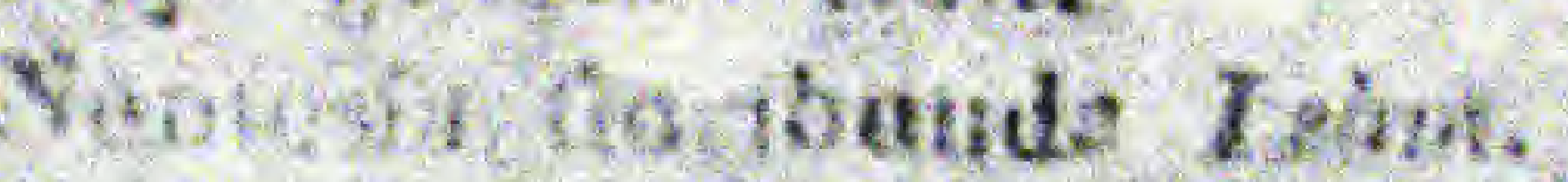

seohor H. Ary.

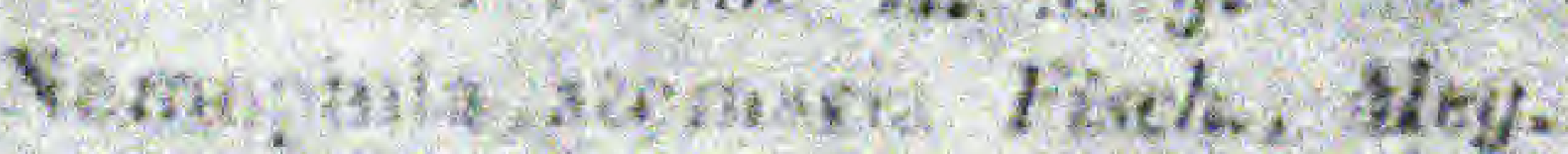

discoidalis.
Nemopliila linifta

Wy

- maculata Be.

- parviflura Donyl.

Nepeta azurea $R$. $B r$.

- botryoides Ait.

- granatensis Boiss.

- grandiflora $\boldsymbol{M B}$.

- lavandủlacéa $\boldsymbol{L}_{\text {. }}$

- macrautha Fisch.

- Meyeri Benth.

Nesiia apiculata Fisch., Mey.

Nicandra physaloides Giaertn.

Nicotiana acuminata $A y h$.

- cerinthoides Hornem.

- commutala Fisch., Mey.

- Langsdorlfii Weinm.

- macrophylia Spr.

- mexicana Schlechtd.

- micrantha $\boldsymbol{H}$. Paris.

$-{ }^{-}$panieurata $\boldsymbol{L}$

- rustica $\boldsymbol{L}$

- Tabacum $L$.

Nolana prostrata $\boldsymbol{L}$.

Nonnea alba $D C$.

Nutobasis syriaca Cass.

Notoceras canariensis $\boldsymbol{R}$. $\mathrm{Br}$.

Ochthodium aegyptiacum $D C$.

Ocimum carnosum Link., Otto.

- graveulens $\boldsymbol{A l}$. Bruun.

- micranthum $W$.

Octadenia Iybica $R$. Br.

Oenothera brachypetala Fisch., Mey.

- chilensis Hort. Paris.

- cognata Fisch., Mey.

- concinna Don.

- densiflora Lindl.

- Iinifolia Nutt.

- Jongiflora $L$.

- macrosiphon Fisch., Mey.

- stricta Ledb.

- tenella Cav.

- triloba Nintt.

Olearia stelluiata $D C$.

Oneorhynchus tenellus Fisch., Hey.

Onobrychis Caput-galli Lam.

- Crista-galli Lam.

Ononis mitissima $\boldsymbol{L}$.

- mollis Savi.

- ornithopodioides $\boldsymbol{L}$.

- serrata Forsk.

- sicula Guss.

Oreomyrrhis eriopoda Endl.

Orlaya platycarpa Koch.

Ormenis mixta $D C$.

Ornithogalum Ecklonii Fisch, . Mey.

Orobus saxatilis Vent.

Oxalis micrantha Bert.

- rosea Jacq.

- villosa $\boldsymbol{M B}$.

Oxylobium ellipticum $R . B r$.

Oxytropis brevirostris $D C$.

Paeonia albiflora Pull.

- anomala $\boldsymbol{L}$.

- tenuifolia $\boldsymbol{I}$

Palafoxia texana $D C$.

Panicum capillare Gron.

- erucaeforme Sibth, Sm. (Urochloa) Zenkowskii Rupr.

Papaver alpioum $\boldsymbol{I}$.

- Argemona $\boldsymbol{L}$.

- argeruonoides Cesult. catieasicum $M B$.

- eroceum Lerlh.

- horrivium $D C$

- orientale $I$.

Pariataria mierantha Lcab.

Taronyelia bisarigasis $7 \mathrm{C}$

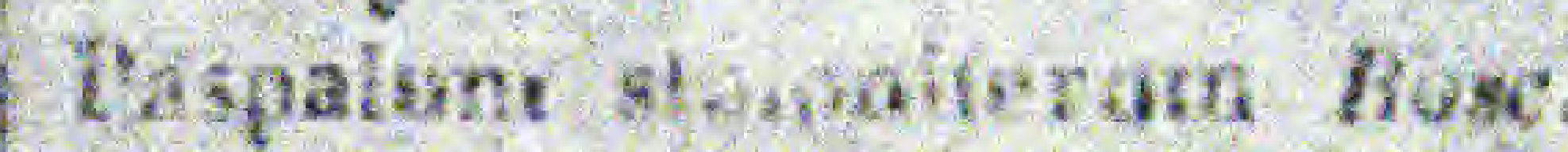

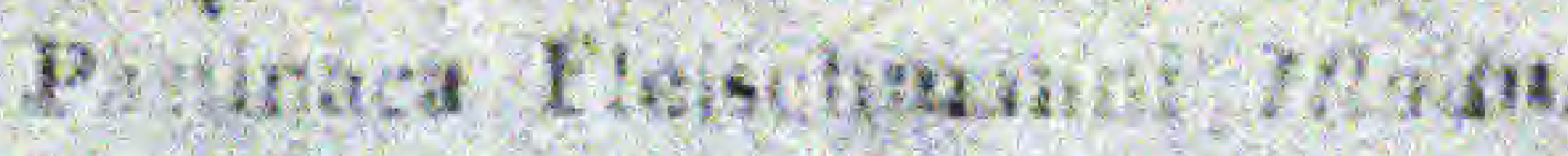

Thargoninca austraw it.
P largonium buniiusum $I I$.

fenniselum cencbroides Rich.

Peutameris airoides Vecs.

Peplis alternifolia $\boldsymbol{M B}$.

- biflora Sulzm.

Petunia Atkinsiana Don.

- longiflora Mey., Rupr.

: - nyctaginiflora Juss. :-

- violacea Lindl.

Phaca frigida $L$.

Phacelia congesta Hook.

- tanacetilolia Benth.

Phacocapuos Cracca Bernh.

Phalacrodiscus arvensis Fisch., Mey.

Phalaris angusta Nees.

- brachystachya Link.

- coerulescens Desf.

Pbarnaceum dichotomum L. $\beta$ fili-

Phlomis agraria Bge.

- alpina Pall.

Phlox-Drummondi Hook.

Phyllanthus obovatus Miihlenb.

Physocaulus nodosus Koch.

Phyteuma limonifolium Sibth, Sm.

- orbiculare $\boldsymbol{L}$.

- Scheuchzeri All.

Picris pauciflora $W$.

Pimpinella rotundifolia $\boldsymbol{M B}$.

Plagiobotbrium rufescens

Plantago amplexicaulis Cav.

- canescens Schrad.

- Hookeriana Fisch., Mey.

- Luefllingii $L$ a 3 caspia

- mexicana Link.

- Psyllium $\boldsymbol{L}$.

- tumida Link.

- virginica $\boldsymbol{L}$.

Platylobium triangulare $R$. $B r$.

Platystemon californicum Benth.

Platystigma lineare Benth.

Plectranthus australis $\boldsymbol{R}$. $\boldsymbol{B r}$. - parvillorus $W$.

Plectritis congesta $D C$.

Plumbago micrantha Ledb.

Pocockia cretica Ser.

Podolepis aurea $C$. A. Mey.

Polycalymna conglutinata $\boldsymbol{F}$. Müller.

Polygala cordifolia $T h b$.

Polygonum argyrocoleum Stend. - salignum $W$.

Polypogon maritimus $W$. - monspeliensis Desf.

- sessilifloras Rupr.

Potentilla approxímata Bge.

- chrysantha Trev.

- gariepensis Eckl.

- kurdica Boiss.

Prasium majus $L$.

Primula scotica Hook.

Psilonema calycinum C. $A$. Mey.

Psilurus nardoides Trin. $\beta$ piptosepalum

Psychine stylosa Desf.

Pterocephalus palaestinus Coult. $\beta$

Pteroneurum graecum $D C$.

Pteropogou chilease Fisch., Mey.

majus.

minus.

Pterostegia drymarioites Fisch, Mey.

Plerotheca bifita- Fisich, Miy.

- nemauseusis fass.

Pulogium micrantbum Clais.

Pulteraea teavifoliz $B . B r$.

Puschicinia scilluides $A d$

Pyrethrum multicante $W$.

Pyerhopappus cinylitiaus oC.

Rasunculus lomatias arpos $F$, Meth

Ranunculus oreophilus $C, A, M_{e y}$

- Schraderianu. Fisch., My.

- Irachycarpus Fisch., Mey.

- trilobus Desf.

Rhagadiolus edulis $W$.

- Hedypnois Fisch., Mig.

- stellatus $\boldsymbol{W}$

-Rhodanthe Manglesii Lindl.

Rhynchopsidium sessiliflorum $D C$.

Richardsonia scabra $\boldsymbol{L}$.

Rochelia leiocarpa Ledb.

Rostellaria abyssinica $A l$. Braun.

Rulingia corylifolia Grah.

Rumex Brownianus Campd.

- bucephalophorus $\boldsymbol{L}$.

- Gmelini Turcz.

- parviflorus Fisch., Mey.

- reticulatus Bess.

- songaricus Schrenk.

- ucranicus $\overline{\boldsymbol{F}}$ isch.

- vesicarius $\boldsymbol{L}$.

Sagina decumbens Elt.

- saxatilis $I \mathrm{Vimm}$.

- stricla Fries.

- subulata Presl.

Salvia controversa Ten.

- grandiflora EtIl.

- nilolica Juss.

- - $\beta$ Schimperiana.

- plebeja $R$. $B r$.

- rhombifolia Ruiz., Pav.

- verticillata $L$. $\delta$ submutica.

Sarrolus litoralis $\boldsymbol{R}$. $\boldsymbol{B} \boldsymbol{r}$.

Sanguisorba alpina Bge.

- media $\boldsymbol{L}$.

- tenuifolia Fisch.

- - $\beta$ n. rubris.

Santolina squarrosa $W$.

Saponaria cerastoides C. A. Mey.

- orientalis $\boldsymbol{L}$.

- porrigens $\boldsymbol{L}$.

Sarracha viscosa Schrad.

Saxifraga orientalis Jacq.

- rotundifolia $L$.

Scabiosa caucasica $\mathrm{MB}$.

- micrantha Desf.

Scandix brachyearpa Guess.

- iberica $M B B$.

- macrorbyncha Fisch, Hey.

- persica Wart.

- pinnatiflda Vent.

- - $\beta$ sungarica.

Schismus marginatus $P$. de $B$.

Schistanthe peduncularis Kunze.

Schmidtia coronopifolia Cass.

Scleropus amarantoides Schrad.

Scrofularia divaricata Ledb. 
Siča Thunbifolia $L$. sprinosa $\boldsymbol{L}$. iriloba $L$. vesicaria Cav.

Siegesbeckia flosculosa Herit. himatayensis $\boldsymbol{H}$. Berl.

- orientalis $\boldsymbol{L}$.

Silene aprica Torcz.

- Bergeri Schott. compacta Fisch. dicholoma Ehrh. fimbriata Sims. fruticosa $\boldsymbol{L}$. hirsuta Lag. $\beta$ asturica. integripetala Bory, Chaub. ornata $A i t$. pendula $L$ portensis $\boldsymbol{L}$. (S. bicolor Thore). praecox Link.

- pygmaea Link. pyriformis Hort. viscida Haberl.

Silybum marianum Guertn.

Sinapis Allionii Jacq.

- bispida Schousb.

- juncea $\boldsymbol{L}$.

Sisynbrium binerve $C$. A. Mey.

- brachypetalum Fisch., Mey.

- bursifolium $\boldsymbol{L}$.

- Cumingianum Fisch, Mey.

- incanum Bernh.

- persicum Spr.

- pumilum Steph.

- villosum Lag.

Sium medium Fisch, . Hey.

sisaroideum $D C$.

Sogalgina trilobata Cass.

Solanum Balbisii Dun.

- ciliatum Lam.

Soliva anthemifolia $\boldsymbol{R} . \boldsymbol{B r}$.

- Barklayana $D C$

Sollya heterophylla Lindl.

Sorema paradoxa Lindl.
Specularia biflora Fiech, Mey

Spermacoce tenuior $L$.

- $\quad \rightarrow$ Iatifolia.

Spbacele subhastata Bentit.

Sphaerolobium vimineum Sit.

Sphaerostigma Chamissonis Fisch.

- hirtum Fisch., Mey.

- minutiflorum Fisch., Mey.

- strigulosum Fisch., Mey.

Sphenogyne adoniditolia $D C$.

- anthemoides $\boldsymbol{R}$. $\boldsymbol{B r}$.

- microcephala $D C$.

- speciosa Harris.

Spilanthes fusca $\boldsymbol{H}$. Paris.

- leucucephala H. Berol.

- oleracea $\boldsymbol{L}$.

Stachys circinnata Herit. setifera C. A. Mey.

Stellaria borealis Bigel.

Stemodia parviflora Ait.

stipa tortilis Desf.

Stobaea heteropbylla $T h b . \beta$ radiata.

Streplocarpus Rexii Lindl.

Succowia balearica Medic.

Sutherlandia frutescens $\boldsymbol{R}$. $\boldsymbol{B r}$.

Syuedrella nodiflora Gaertn.

Tagetes signata Bartl.

Tanacetum globiferum $D C$.

Tauscheria gymnocarpa Fisch.

Tetragonia echinata Ait.

- expansa Ait.

Tetrapoma barbareaefolium Turcz. Thalictrum baicalense Turcz.

- kemense Fries., Kamtsch.

- squarrosum Sieph. (trigynum Fisch.)

Thelygonum Cynocrambe $L$.

Thermopsis fabacea $D C$.

Thlaspi ceratocarpum Hiur. platycarpum Fisch., filey.

Thomasia discolor Steud.

- purpurea Gay.

Thuja occidentalis $\boldsymbol{L}$.

Tillaea connata Ruiz., Pav:-
Tillaea muscosa $\boldsymbol{L}$.

Tittsonia tagetillora Desf.

Tofieldia calyculata $W$ ahient.

Tolpis altissima Pors.

Tommasinia serticillaris Bertol.

Tordyliurn apulum $\boldsymbol{L}$.

- syriacoen $L$.

Tradescantia Crassula Link., Otto.

Trichogyue virtitillata less.

Trichonema ramiflorum Sweet.

Trifolium circumdatum Funze.

- diffusum Ehrh.

- erinaceum $M B$.

- filiforme L., Sm.

- latinum Sebast.

- leacanthum $\boldsymbol{M B}$.

- nidificum Grisb.

- oxypelalum Fisch., Mey.

- physanthum Hook.

- Sebastiani Savi

- suaveolens $W$.

- tomentosum $L$.

- trichocephajum $M B$

- Iridentatum Lindl.

Trigonella arcuata C. A. Mey.

- calliceras Fisch.

- cancellata Desf.

- ensifera Trautv.

- giumerata $\boldsymbol{H}$. Puris.

- urthuceras Kur., Kir.

- spinusa $\boldsymbol{L}$.

- torta Sm.

Tripleris cheiranthifolia $S$ chultz.

Trisetum myrianthum Fisch., Mey.

- neglectum Röm., Schult.

Triticum orientale $M I B$.

- prostratum L. fil.

- subtile Fisch., Mey.

- villosum $\boldsymbol{P}$. de $\boldsymbol{B}$.

Trollius altaicus $C$. A. Mey.

Tropaeolum tricolor Sweet.

Tunica prolifera Fisch, Mey.

- stricta Fis $h$., Mey.

Turgenia latifolia Hoffm.

Urosperinum picroides Desf.
Crlica cubensis $h$

bodartii $t$.

elongala tin

- grandidentaia Moris.

- mesicana H. Beroi.

- pilulifera $L$.

Vaillantia muralis

Vaierianella auricula $t 4$

- brachystephana

- echínata DC.

- eriocarpa Dest

- hamata Brist.

- plagiostephana Fisch, Mey

- pumila $D C$

- sclerocarpa Fisch, Mey

- Szovitsiana Fisch,, Miey.

- vesicaria Mỏnch.

Verbascum Wallichianum Benth.

Verbena biserrata Kunth.

- hispida Ruiz., Pav

- supina $\boldsymbol{L}$.

Veronica bitoha $\boldsymbol{L}$.

- sibirica $\boldsymbol{L}$.

Vicia cinerea Stev

- grandiflora Scop.

- megalosperma $M B$.

- sordida Tialdst., Fit.

- villosa Roth. $\beta$ glabrescens.

Viola Gmeliniana Röm., Schutl

- occulta Letim.

- palmensis B. Hebt.

Wachendorfia thyrsiflora $L$.

Wahlenbergia exilis Alph. DC

- gracilis Alph. DC.

- Jobelioides Alph. Dt

- parvillora Alph br.

Westringia tongifotia $\boldsymbol{H}-I^{2}$.

Wiedemannia osientalis $\mathrm{Fise}$, Brey Xeranthemun erecium presl. - inaprerlum $\boldsymbol{L}$.

Zazintha verrucosa Gaertn.

Ziziphora capitala $\boldsymbol{L}$.

\section{Semina in regione wolgensi, prope sarepta lecta.}

Achillea Gerberi $W$.

- leptophylla $\boldsymbol{M B}$.

Alhagi camelorum Fisch.

Allium tulipaefolium Ledb.

Anabasis aphylla $L$.

Arguzia Messerschmidia Stev.

Artemisia monogyna Waldst., Fit.

Astragalus longiflorus Pall.

- vulpinus $t /$

Atriplex verrucifera $\boldsymbol{M B}$.

Brachylepis salsa C. A. Mey. -

Bulliarda Vaillantii $D C$.

Bunium luteum Hoffm.

Calophaca wolgarica Fisch.

Camphorosma annuum Pall.

Centaurea adpressa Ledb.

- arenaria $\mathbf{M B}$.

Chartolepis glastifolia Cass.

Chondrilla graminea $\mathbf{M B}$.

- latifolia $M B$. $\beta$ acanthophylla.

Cirsium esculentum C. A. Mey.

- setigerum Ledb.

Cólpöłium bulbosum Trin.

Corispermum byssopifolium $\boldsymbol{L}$.

- Marschallii Stev.
Corispermum nitidum Kit.

Crambe aspera $\boldsymbol{M B}$.

Cyperus glomeratus $\boldsymbol{L}$. - patulus Kit.

Delphitium puniceum Pall.

Dianthus capitatus $D C$.

- pallidiflorus Ser.

- pratensis $M B$.

- rigidus $\boldsymbol{M B}$

- squarrosus $M B$.

Epheảra mỏnostáchya $\boldsymbol{L}$.

Eragrosicis suaveolens Becker.

Eriosynaphe Iongifolia $D C$.

Erysimum versicolor Andrz.

Euphorbia tenuifolia .AB,

- undulata $\boldsymbol{M B} \boldsymbol{B}$.

Ferula caspia $\boldsymbol{M B}$.

Fritillaria ruthenica Wikstr.

Galium tataricum Trev.

Geranium Iongipes $\boldsymbol{D C}$.

GrammopetalumHoffmanni C.A.Mey. (Trinia MB.)

Halimocnemis glauca $C$. A. Hey.

- monandra $C$. 4. Vey.

Halocnemum strobilaceum $M B$.
Halogeton monandrus $C, A$. Mey. Herniaria odorata Andrz.

Iris aequiloba $L e d b$.

Jurinea Eversmanni $\boldsymbol{B} g$.

Lactuca sagittata Kitt.

(L. altissima $M B$.)

Lasiospora tuberosa Fisch.

Lathyrus incurrus Roth.

Leovurus glaucescens $\mathrm{Bge}$.

Linaria odora Chav.

Linosyris divaricata $\boldsymbol{D C}$.

- Cataricata Less.

Lythrum nanum Kar., Kir.

- TL rmifolia $\boldsymbol{M B}$.

- tribracteatum Salsm.

Miedicago cancellata $\mathbf{M B}$.

Melilotus ruthenica $\boldsymbol{M B}$.

Nitraria :choberi $\boldsymbol{L}$

Onosma tinctorium $\mathbf{B B}$.

Palimbia salsa Bess.

Peucedanum latifolium $D C$. - sibiricurn $W$.

Pharnaceum Cerviana $\boldsymbol{L}$.

Podosperinum canum $C$. A. Tey. - molle Fisch., Mey.
Pulegium micranthum Claas.

Pyrethrum millefoliatom $H$

Rindera tetraspis Pall.

Rumex Marschallianos Reht

- steuophyllus Ledb.

Salsola brachiata Pall.

- clavifolia Pall.

- laricina Pall.

- Soda $\boldsymbol{L}$

- tamariscina Pall.

Scabiosa ucranica $\boldsymbol{L}$

Schoberia baccifera C. A. Mez

- maritima C. A. Mey.

Scorzonera Marschalliana C, A. Mey.

Secale fragile $\boldsymbol{A J B}$.

Serratula Gmelini Ledb.

- xeranthemoides $M B$

Silene procumbens Ajurr.

Sisymbrium junceum $\boldsymbol{M B}$.

Syrenia Lamarckiaba Andrz.

Tragopogon ruthenicus Bess.

Triticum desertorum 'Fisch, -

Tulipa Biebersteiniana Scliklt

- billora Pall.

- suareolens- $I$.

\section{A. Meyer.} praefectus hort.

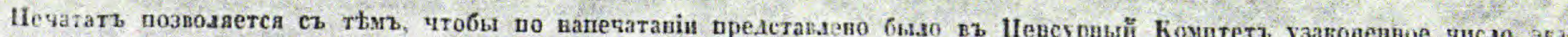




\section{Semina selecta, e collectione anni 1832, quae hortus botanicus Imperialis Petropolitanus pro nutua commutatione offert.}

Acalypha brachystachya Hornem. Allium strictum Sehrad. pancifora Hornem. A antbocephalus amplexifolius $\boldsymbol{K a r}$. $\quad$ Victorialis $\boldsymbol{L}$.

Acanthospermum humile $D C$. et Kir. Alonsoa caulialata Ruiz., Pav. Achille a filipendulina Lam. - mongolica Fisch. Achyracbaena mollis Schauer. Acinos alpinus Pers.

- graveolens Link.

- origanifolius $R$ upr. rotundifolius Pers.

A cuida tulierculata Moq. Tand. Aconitum oebotense Rchb. Acroglochils chenopodioides Schrad. A.tinomeris Felianthoides $D C$.

Jero, tora denticulata Fisch. var.

Aleser maricata $D C$. latifoli

A

- Commea Jacq. var. anomala.

- squarrosa Stev.

- veraalis $\boldsymbol{L}$.

ps cyliodrica Host.

ovata $t$.

spelloides Tausch.

squarrusa L, Schreb.

triaristata $W$.

triuncialis $\boldsymbol{L}$., $\boldsymbol{W}$.

ventricosa Tausch.

A cgopodiou alpestre Ledb.

Aerpi lemata Juss.

Aetbionewa Bubaunii Dr heterocarpum Gay.

A gasy lis caucasica $\boldsymbol{S}_{\boldsymbol{\mu}}$.

Agriza aia pilosa Ledb.

Agrostis lachnantha Nees, ab Es.

- Diteni Guss.

- patida DC

retworacta 14

Alows orthia cordats Boiss.

Es capilaris Hos!

Cupaniana tass.

upsis pulchella Ten.

4he besutitid alpina $\mathrm{t}$.

arvensis $S_{\text {cop. }}$.

fivas Srtinmm., Wimm.

phatsens $M P$.

Nepis Far. et Fir

Vlata Fise in, Mes un teris.

hybrenorbizizis $r d a$.

sidicuan $\boldsymbol{T}$.

p)
Alopecurus utriculatus Pers.

Alsine arabica Fisch., Mey.

- globulosa C. A. Mey.

- pharnaceoides. Fenzl.

- propinqua R. $B r$.

- rubella Wahlb.

Alternanthera sessilis R. $\boldsymbol{B r}$.

Althaea hirsuta ${ }^{-}-\beta$ caspia.

Alyssum confertum H. Berol.

- micranthum C. A. Mey.

- Szovitsianum Fisch., Mey.

- umbellatum Desv.

Amarantus albus $L$.

- melancholicus L. $\beta$ tricolor $\boldsymbol{L}$.

- retroflexus $L$.

- spinosus $\boldsymbol{L}$.

- sylvestris Desf.

Amberboa Lippii $D C$.

- moschata DC.

- muricata $D C$.

Amblyogyna polygonoides Rafin.

Amellus annuus $W$.

- tridactylus $D C$.

Amethystea coerulea $\boldsymbol{L}$.

Ammannia difiusa $\boldsymbol{W}$.

- latifolia $\boldsymbol{L}$.

- sanguinolenta Sw.

- Wormskioldii Hort. Berol.

Amsinckia angustifolia Lehm.

- intermedia Fisch., Mey.

- lycopsioides Lehm.

- spectabilis Fisch., Mey.

Anacyclus Pyrethrum DC.

Anarrbinum bellidifolium $\boldsymbol{W}$.

- duriminium Pers.

Anastatica hierochuntica $\boldsymbol{L}$.

Anchusa capensis Thbg.

- crispa Viv.

- ochroleuca MB.

- stylosa Stev,

Ancistrocarpus maypurensis Humb.,

Androsace Gmelini Goerto.

Bonpl. Arenaria conimbricensis Brot.

- emarginata Brot.

- lactifiora Fiseh, grandiflora. - montana $\boldsymbol{L}$.

Andryala cheiranthifotia Hert?

- integrirolia $X$.

- laxillora Dr.

nemote narcis inora $\boldsymbol{L}$.

Angelica anomole $t e b$ ?

Anglathes aticolius C. A. MES.

Anthemis chia $L$.

- chrysantha Gay.

- nobilis $L$.

- $\quad$ - $\beta$ discoidea.

- peregrina $L$.

- retusa Delile.

- Triumfetti All.

Anthoxanthum ovatum Lag.

Anthriscus tenerrima Boiss.

- trichosperma Schult.

Anychia capiliacea $D B$.

- dichotoma Mich.

Aquilegia advena Fisch. Mey.

- elata Ledb.

- fragrans Benth.

- glandulosa Fisch.

- jucunda Fisch., Lall.

- pubiflora Lindl.

- Skinneri Hook.

- sibirica Lam.

- viridiflora Pall.

- Wittmanniana Stev.

Arabis alpina $L$.

- ambigua $D C$.

- bellidifolia Jacq.

- ciliata R. Br.

- collina Ten.

- declinata Schrad.

- Eschscholziana Andz.

- lilacina Schrad.

- pendula $L$.

- saxatilis $\mathbf{A l l}$.

- stenopetala $\boldsymbol{W}$.

- verna $R . B r$.

- aor vegica Gunn.

pathulata Desf.

Argenawis albiflora Hornem.

- mexicana $\boldsymbol{x}$. ochtolewer Sicect. ria ty ein tichit boiss.
Anisoderis rhoeadifolia Fisch., Mey. Artemisia Mutellina Vill. Anisolotus anthylloides Bernh. Asparagus caspius $\boldsymbol{H}$. Vind. - Wrangellianus Bernh. Anoda cristata Schldl.

- heteromalla Schrad. $\alpha$ erio-

carpa.

3 leiocarpa

- Indoviciana Fisch., Mey.
Asphodelus ramosus $\boldsymbol{L}$.

Aster salsuginosus Richards.

- sibiricus $\boldsymbol{L}$.

Asterolinum adoënse Kunze.

- stellatum Link.

Astragalus adsurgens $\boldsymbol{P}$ all.

- Aegiceras $W$.

- brachyceras Ledb.

- contortuplicatus $\boldsymbol{L}$.

- cymbaecarpos Brot.

- epiglottis $\boldsymbol{L}$.

- juvenalis Del.

- pentaglottis $\boldsymbol{L}$.

- reticulatus $M B$.

- sanguinolentus $M B$.

- scorpioides Pourr.

- sesameus $\boldsymbol{L}$.

- uliginosus $\boldsymbol{L}$.

Astrantia Biebersteinii Trautv.

- carniolica Jacq.

Astrocarpus sesamoides $D C$.

Aubrietia deltoidea $D C$.

Aulacospermum anomalum Ledb.

Axyris amarantoides $L$. - hybrida $L$.

Baeria chrysostoma Fisch., Mey.

Baptisia australis $\boldsymbol{R}$. Br.

Barbarea arcuata $R c h b$.

- orthoceras Ledb.

- prostrata Gay.

Barkhausia alpina $D C$.

Bartonia aurea Lindl.

Begonia Dregei Dietr., Oito.

Bellevalia ciliata Nees ab. Es.

Bellis integrifolia Mich.

Betckea samolifolia $D C$.

Bidens connata Müihlenb.

- frondosa $\boldsymbol{L}$.

- parviflora $\boldsymbol{W}$.

Bifora radians $M B$.

- testiculata Spr.

Biotia latifolia $D C$.

Biserrula Pelecinus $L$.

Blumeo bachia insiguis Sehrad.

Boissiera bromoides Rochst.

Bossiaea thymifolia Hort. angl.

Bothriospersum tenellum Fisch.,

Rotryadenia ismelinl Ftsch, Mey.

botryanthus micranthes $\boldsymbol{H}$. Petr. Erovit fit C.A. Moy.

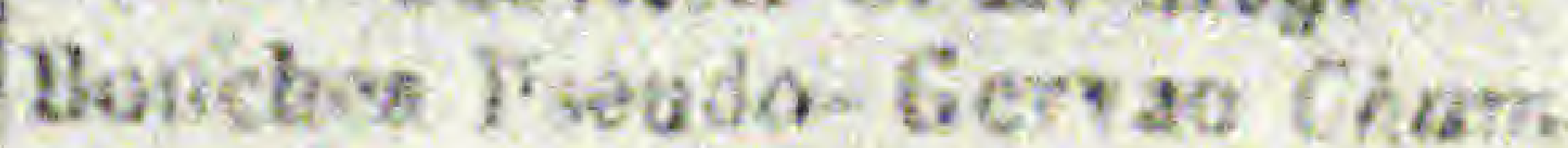


Bowlesia tenera Spr.

Boykinia aconitifolia $\mathrm{N} n \mathrm{t}$.

Brachycome diversifolis Fisch., Mey. - iberidifolia Benth.

Braya siliquosa $B g e$.

Bromus brizeeformis Fisch., Mey.

- conferlus M. B. c. varr.

- oxyodon Schrenk.

- racemosus I. c. Tarr.

- vestitus Schrad.

Broteroa trinervata Pers.

Browallia elongata Humb., Kunth.

Buffonia annua $D C$.

Bulbine annua $w$.

- Hookeri Kunth.

- semibarbata $R$. Br.

Cacalia hastata $\boldsymbol{Z}$.

Calamintha umbrosa Fisch., Mey.

Calandrinia Menziesii Hook.

- micrantha Schlechtd.

- pilosiuscula $D C$.

- procumbens Moris.

Caiceolaria glutinosa Regel.

- pinnata $\boldsymbol{L}$.

Calendula brachyglossa Rupr. (bicolor Boiss. pl. Kotsch.)

- Crista Galli Yis.

- cristata Guss.

- gracilis $D C$.

- parviflora Guss. (sicula W)

- sicula Cyr., DC. (ceratosperma Vis.)

Calepina Corvini Desv.

Calimeris biennis Ledb.

- platycephala Cass.

Callicephalus nitens C. A. Mey. Callichroa platyglossa Fisch., Mey. Calliglossa Douglasii Hook., Arn. Campanula barbata $L$.

- lamiifolia M. B.

- Langsdorffiana Fisch.

- Loefuningii Brot.

- pusilla Haenke.

- Steveni $\boldsymbol{M}$. $\boldsymbol{B}$.

- thyrsoidea $L$.

Carduus seminudus $\boldsymbol{M}$. $\boldsymbol{B}$.

Carex atrata $\boldsymbol{L}$.

- Gmelini Hook., Arn.

- Jeiorhyncha C. A. Mey.

- lupulina Muehlenbg.

- rhynchophysa $\boldsymbol{C}$. $\boldsymbol{A}$. Mey.

- stipata Muehlenbg.

Carrichtera Vellae $D C$.

Caryodaphne Browniana Nees ab Cleome fug

Caylusia abyssinica Fisch, Mey.

Ceanothus azureus Desf.

Cedronella breviflora C. A. Mey.

Celosia trigyna $\boldsymbol{L}$.

Celsia Arcturus $L$. $\beta$.

$\rightarrow$ orientalis $L$. -

Cenia geminata Kunze.

Centaurea involucrata Desf.

- Kartschiana Scop.

- leptacantha Fisch. Mey.

- maculosa Lam. var.

- orientalis $\boldsymbol{L}$.

- ovina Pall.

- rupestris $L$. a subinermis.

- semperrirens $\boldsymbol{L}$.

- tenuiramea Del.

- trichocephala W. var. lati- Conoclinum janthinum.

Centranthus dasyarpus $K$ ze. folia.

- macrosiphon Boiss.

Centratherum intermedium Less.

Cephalaria procera Fisch., Lall.

- tatarica $R$. et $S c h$.

Cephalophora aromatica Schrad.

Ceraslium anomalum $\boldsymbol{W}, \boldsymbol{K}$ it.

- infatum Link.
- campanulatum Viv.

- chloraefolium Fisch., Mry.

- dichotom. 1 . $\gamma$ glandulostura.

- furcalum Cham.

- latifolium $\boldsymbol{L}$.

- microspermum C.A. Mry.

- multiflorum C. A. Mey.

- perfoliatum $L$.

- pilosum Ledb.

- purpurascens Adam.

- tetrandrum $S m$. $\beta$ asturicum Fisch., Mey.

Ceratocapnos umbrosa Durieu.

Ceratocephalus falcatus Pers.

- orthaceras DC.

Cercodia erecta Murr.

Chaenostoma aethiopicum Benth.

- foetidum Benth.

- polyanthum Benth.

Chaerophyllum maculatum $\boldsymbol{W}$.

- Prescottii DC.

Chaetonychia cymosa Welwo.

Chaeturus fasciculatus Link.

Chamaepeuce Casabonae $D C$.

- stricta $D C$.

Chardinia xeranthemoides Desf.

Charieis heterophylla Cass.

Chlamydophora tridentata Ehrenb.

Chloris meccana Hochst., Steud.

- radiata $S v$.

- submutica Humb., Kunth.

- truncata $R$. Br.

Chlorophytum inornatum Gavl.

Chondrilla brevirostris Fisch., Mey.

Chrysanthemum pinnatifidum $\boldsymbol{L}$. fil.

Cineraria Iobata Herit.

Cinna pendula Trin.

Cirsium arachnoideum $\boldsymbol{M} . \boldsymbol{B}$.

- dealbatum $\boldsymbol{M}$. $\boldsymbol{B}$.

- munitum $\boldsymbol{M}$. $\boldsymbol{B}$.

- obyallatum $\boldsymbol{M} \cdot \boldsymbol{B}$.

- Wiedemannianum

Cistus creticus $L$.

Fisch.

- garganicus Ten.

- tauricus Presl.

- undulatus Dun.

- vaginatus Ait.

Cladanthus pedunculatus Durieu.

Claytonia alsinoides Sims. Il: albo.

- perfoliata Donn.

ab Cleome fugax Schrad.

- pungens $W$.

- Steveniana Schult.

- violacea $L$.

Clerodendron calamitosum $\boldsymbol{L}$.

Clintonia pulchella Lindl.

Clypeola microcarpa Hort.

- Cnidium dahuricuñ Fisch., Mey.

Coix Lachryma $\boldsymbol{L}$.

Colchicum speciosum Stev.

- umbrosum Stev.

Collinsia bicolor Benth.

- grandiflora Lindl.

- parviflora Lind.

- sparsiflora Fisch., Mey.

Conioselinum coenolophioides Turcz.

- Fischeri Wimm., Grab.

Conringia planisiliqua Fisch, Mey.

Convolvulus farinosus $L$.

Coreopsis auriculata $\boldsymbol{L}$.

- coronata Hook.

- Drummondi Torr., Gray.

Cornucopiae cucnllatum $L$

Cornus suecica $\boldsymbol{L}$.
Coronilla montana Scop.

Cortusa Mattbioli $l$.

Corydalis claviculata $D C$. nobilis Pers.

Cosmanthus fmbriatus Nolte. viscidus A. DC.

zula anthemoides $L$.

- coronopifolia $\boldsymbol{L}$.

- filifolia $T h b$.

- microcephala $D C$.

- pusilla Thb.

- sororia $D C$.

Crassula lanceolata Endl.

- pubescens Endl.

- Schimperi Fisch., Mey.

- Columpae Froel.

- multicaulis Ledb.

- sibirica $L$.

Crocus speciosus $\boldsymbol{M}$. $\boldsymbol{B}$.

Crotalaria impressa Nees ab Es.

- incana $\boldsymbol{L}$.

- parviflora Roth

Cryptantha glomerata Lehm.

- microcarpa Fisch., Mey.

Cryptostemma arctotideum Rupr. (Alloizonium Kze.)

- calendulaceum $\boldsymbol{R}$. Br.

- hypochondriacum $\boldsymbol{R} . \boldsymbol{B r}$.

- Iusitanicum Welro.

Cucumis Figarei Del.

- prophetarum $\boldsymbol{L}$.

Cuphea silenoides Nees.

Cyclanthera pedata Schrad.

Cynoglossum furcatum Wall.

- glochidiatum Wall.

Cysticapnos africana Gaertn.

Dactylaena micrantha Schrad.

Dahlia Merkii Lehm.

Daucus'glochidiatus Fisch., Mey.

- monteridensis Link.

Deeringia baccata Mog. Tand,

Delphinium cardiopetalum $D C$.

Hey. $\quad$ - cheilanthum Fisch

- grandiflorum $\boldsymbol{L}$

- lasiostachyum Fisch., Mey.

- triste Fisch.

Desmanthus virgatus $W$.

Desmodium canadense $D C$.

Dianella atrata Fisch.

Dianthus Cyrii Fisch., Mey.

Diastrophis cristata Fisch, Mey.

Dichelostemma congestum Kunth.

Dichrocephala sonchifolia $D C$.

Dicliptera resupinata Juss.

Didiseus coeruleus Hook.

Dimetopia hirta Benth.

- isocarpa Bartl.

Diplophyllum veronicaeforme Stev.

Dischisma capitatum Chois.

- clardestinum $\boldsymbol{E}$. Mey.

Dodecatheon integrifolium Mich.

Doronicum caucasicum .M. B.

Draba Aizoon Wahlenb.

- alpina $\boldsymbol{L}$.

- arabisans Mich.

- aurea Vahl.

- borealis $D C$

- caroliniana Walt

- dasycarpa C. A. Mey.

- hirta $L$.

- - dovrina.

- lactea Adams.

- muricella Wahlenb.

- rupestrîs $R$. Br.

- scandinarica Lindbl.

- $\quad$ - $\beta$ coronopifolia.

- tridentata $D C$.

Dracocephalum argunense Fisch.

Dracocephain

- parvif

- tbymiflorum $z$

Drummondia nitellows

Echinospermum cousang uine

- cristatum Bong. Wisch, Mey.

- deflexum Lehm.

- Diploloma Schrenk.

- heteracanthum Ledb.

- Karelini Fisch, Mey.

- laeve C. A. Mey.

- patulum Lehr.

- Redowskit Retni.

Echiom arebarinm Guss.

- creticum $x$

- italicum $\boldsymbol{L}$.

- plantagioerim $L$.

- rubrum Jucq

Elaeagnus parvifolis Soc.hort. $\mathrm{tm}$.

Elephanthopus caroliniamus $W$.

Eleusine coracana Pers.

- indica Gaertn.

- oligostachya $\boldsymbol{L}$ ink.

Elsholtzia cristata $\boldsymbol{W}$.

Elviria biflora $D C$.

Elymus Caput - Medusae $\boldsymbol{L}$.

- crinitus Schreb.

Emex spinosa Campd.

Enarthrocarpus lyratus $D C$.

Epilobium algidum $\boldsymbol{M}$. $\boldsymbol{B}$.

- alpinum $\boldsymbol{L}$. et Fries.

- angustifolium $\boldsymbol{L}$. 3 parvifio

- chilense $\boldsymbol{H}$. Berol. rum $\mathcal{C}$. A. Mey.

- Durieui Gay.

- latifolium $L$. var.

- mexicanum Ifort.

- minutum Lindl.

- nepalense James.

- origanifolium Lam. var. ro sulatum $B x p r$.

- palustre $\boldsymbol{L}$. albiflorum.

- repandum Spach.

Eragrostis aegyptiaca Link.

- ciliaris Link.

- mexicana Link.

- peruviana Trin.

- plumosa Link.

- Purshii Fisch., Mey.

Eranthemum variabile $\boldsymbol{R}, \boldsymbol{B r}$.

Erechtites carduifolia $D C$.

- hieracifolia Rafin.

- valerianaefolia $D C$.

Eremegone graminifolia Eensl. 
Erysimum sisymbrioides $C$. A. Mey. Geraniam platypetalum Fisch., Mey. Hordeum jubatum $\boldsymbol{L}$.

Erythronium Dens canis $L . \quad-$ pyrenaicum $L$.

Eucharidium eoncinnum Fisch., Mey. - sibiricum $\boldsymbol{L}$.

- grandillorum Fisch, Mey. Geropogon glaber $\boldsymbol{L}$.

Euclidium syriacum $\boldsymbol{R}$. $\boldsymbol{B r}$,

Eupatorium calaminthaefolium

Euphorbia aspera $\boldsymbol{M}$. $\boldsymbol{B}$.

- nutans $\boldsymbol{H}$. Paris.

- geniculata Orteg.

- graeca Boiss.

- Lagaseae $S p r$.

- Lathyris $\boldsymbol{L}$.

- micrantha Steph.

- Myrsinites $\boldsymbol{L}$. $\boldsymbol{\beta}$ minor.

- Pinea Bertol. (

- Pithyusa $\boldsymbol{L}$.

- procera $\boldsymbol{M}$. $\boldsymbol{B}$.

- pterococca Brot.

- pubescens Vahl...

- trigonocarpa Fisch.

- virgata $\boldsymbol{W}$. et Kit.

Eutoca Wrangelliana Fisch., Mey. Eutriana curtipendula Trin.

Euxolus caudatus Moq. Tand.

- emarginatus Al.Br.et Bouché.

- lividus Moq. Tand. viridis Moq. Tand.

Erax asterisciflora Pers.

Farsetia eriocarpa $\boldsymbol{D C}$.

Fedia graciliflura Fisch., Mey.

Festaca divaricata Desf.

- pectinella Del.

- procumbens Kunth.

- rigida Kunth.

- $\quad$ - $\beta$ hemipoa Del.

- tenuiflora Schrad.

- unioloides Kunth.

Florestina pedata Cass.

Forskohlea angustitolia Retz.

Fongerouxia recta $\boldsymbol{D C}$.

Erancoa ramosa Don.

Frittllaria pallidiflora Sehrenk.

Froelichia gracilis Moq. Tand.

Eumaria agraria $\mathbf{L}$ ag.

- alexandrina Ehrenb.

- anatolica Boiss.

- capreolata $\boldsymbol{L}$.

- muralis Sonder.

Gagea taurica Stev.

Galactites tomentosa Moench.

Galatella dahurica $D C$.

- Hauptii Lindl. \& tenuifolia.

Galega orientalis Lam.

Galinsogea brachystephana H. Berol.

Galium caudatum Boiss.

- intricatum Reut.

- retrorsum $D C$.

- tenuissimum $\boldsymbol{M}$. $\boldsymbol{B}$.

- valantioides $M$. $B$.

- verticillatum Danth.

Gamo'epis Tagetes $D C$.

Gariciella Nigellastrum $\boldsymbol{L}$.

Gastridium australe $\boldsymbol{P}$. Beauv.

Gastrocotyle hispida Bge.

Gatyona globulifera Cass.

Gandinia fragilis $P$. Beauv. geminiflora Gay.

Gaura parvifiora Hook.

Gaya simplex Gawd.

Gelasia villosa Cass.

frenista candicans $\boldsymbol{L}$.

Geutiana Amarella $\boldsymbol{i}$.

$\rightarrow$ asclepiadea $\mathbf{L}$

- germanica W.

t- macrophyll páli.

- pannonica Scup.

Geranium affine Ledb. * is:

- albillormen Ledb.

- coeruleum Patr.

- Londesil Fisch.
Hyacinthus amethystinus $\boldsymbol{L}$.

Hydrastis canadensis $\boldsymbol{L}$.

Hymenocarpus nummularius $W$.

Hymenoxis californica Hook.

- mutica Torr., Gray.

Hyoscyamus albus $L$.

rubelium $\boldsymbol{H}$. Petr.

strictum Ait.

Gilia millefoliata Fisch., Mey.

Glossocomia clematidea Schrenk.

Glycosmis citrifolia $\boldsymbol{L}$ indl.

Gnaphalium cephaloideum $\boldsymbol{W}$.

- citrinum Hook., Arn.

- resedaefolium Trev.

Goldbachia tetragona Ledb.

Grangea maderaspatana Poir.

Gratiola latifolia $R$. $B r$.

Grewia parvillora Bge.

Grindelia integrifolia $\boldsymbol{D} \boldsymbol{C}$.

- mexicana Kze.

- pulchella Dun.

Guizotia oleifera $D C$.

Gunnera seabra $R$. et Pav.

Gynandropsis muricata Schrad.

Gypsophila elegans M. B.

- perfoliata $L . \beta$ tomentosa $D C$. - repens $L$.

Hasselquistia cordata Jacq.

Hebenstreitia integrifolia $L$.

Hedeoma hispida Purs $h$.

Hedysarum caucasicum $M$. $B$.

- elongatum Fisch.

- neglectum Ledb.

- spinosissimum $\boldsymbol{L}$.

Helenium autumnale $\boldsymbol{L}$.

- brachyglossum Fisch., Lall.

Helianthemum denticulatum Thib.

- guttatum Mill.

- intermedium Thib.

- lasiocarpum $\boldsymbol{H}$. Paris.

- tauricum Rupr.

- villosum Thib.

Helichrysum foetidum Cass.

- fulgidum $\boldsymbol{w}$.

Heliophila amplexicaulis $\boldsymbol{L}$. fil.

- crithmifolia $W$.

- pendula $\boldsymbol{W}$.

- pilosa Lam. $\beta$ DC.

- pusilla $L$.

- stricta Sonder. angustifolia.

Heliophytum parviflorum $D C$.

Heliophytum parviflorum $D C$.
Helosciadium crassipes Koch. $\beta$ he- Lagascea mollis $C a v$. terophyllum.

- leptophyllum DC.

Heracleum asperum Fisch.

- barbatum Ledb.

- persicum H. Paris.

- pubescens $\boldsymbol{M} . \boldsymbol{B}$.

- villosum Hoffm.

- Wilhelmsii Fisch., Lall.

Herniaria diandra Bge.

- odorala Andrz.

Hesperis tristis $\boldsymbol{L}$.

Heterochroa violacea Walp.

Heterospermum pinnatum Cav.

Heuchera americana $L$.

- cylindrica Dougl.

- divaricata Fisch.

Hibiscus pedunculatus Cav.

- ternatus Cav.

Hieracium alpinum $\boldsymbol{L}$.

- andryaloides Vill.

- aurantiacum $\boldsymbol{L}$.

- prenanthoides Vill.

- villosum $\boldsymbol{L}$.

Hippocrepis unisiliquosa $L$.

Hohenackeria buplenrifolis
- orientalis $\boldsymbol{M}$. $\boldsymbol{B}$.

Hypecoum grandiflorum Benth.

Hypericum attenuatum Chois.

- linearifolium Vahl.

- orientale $\boldsymbol{L}$.

- reflexum $\boldsymbol{L}$. fil.

Jasione perennis $\mathbf{L}$ am.

- pectinata Boiss.

Ilysanthes cubensis Fisch., Mey.

Impatiens parviflora $\boldsymbol{D C}$.

- Roylei Walp.

- tricornis Lindl.

Imperatoria Ötruthium-L. :

Indigofera australis $\boldsymbol{W}$.

Inula hirta $\boldsymbol{L}$.

- Oculus - Christi $L$.

- rhizocephala Schrenk.

Johrenia dichotoma $D C$.

Jonopsidium acaule Reichb.

Ipomoea sibirica Jacq.

Iris Gueldenstaedtiana Lep.

- halophila Pall.

- ruthenica Ait.

- sibirica $L$. $\beta$ haematophylla

Isatis oblongata $D C$.

- sibirica Trautv.

Isopyrum fümarioides $\boldsymbol{L}$.

Juncus arcticus $W$.

- vaginatus $\boldsymbol{R}$. $B$ r.

Jurinea polyclonos $D C$.

Jussieua erecta $\boldsymbol{L}$.

Kalbfussia Muelleri Schultz.

Kentrophyllum tauricưm

Koelpinia linearis Pall.

Krubera leptophylla $\boldsymbol{H o} / \mathrm{m}$.

Krynitzkia leiocarpa Fisch., Mey.

Ktenospermum linifolium Lehm.

Lactuca altaica Fisch., Mey.

- cracoviensis Savicz.

Lagoecia cuminoides $\boldsymbol{L}$.

Lagunea lobata $\boldsymbol{W}$.

Lallemantia iberica Fisch., Mey.

- Royleana Benth.

Lamium bifidum Cyr.

Laserpitium gallicum $C$. Bauh.

- hispidum $\boldsymbol{M}$. $\boldsymbol{B}$.

- latifolium $\boldsymbol{L}$.

Lasiospora angustifolia Cass.

Lasthenia obtusifolia Cass.

Lathyrus pisiformis $\boldsymbol{L}$.

- tuberosus $L$.

Laurentia Michelii $D C$.

- tenella $\boldsymbol{D C}$.

Lavatera arborea $\boldsymbol{L}$.

- cretica $\boldsymbol{L}$

- Orbia $\boldsymbol{L}$.

- plebeja Sins.

Leonurus sibiricus $\boldsymbol{L}$.

- tataricus $\boldsymbol{L}$.

Leontice altaica Pall.

Leontodon eaucasicum Fisch.

Lepidium Armoracia Fisch, Mey.

- densiflorum Schrad.

Fisch., - Menzienti DC.
Mey.

- orientalis $W$. var.
- laciniatum DC.

Iberis linifolia $\boldsymbol{L}$.

Lepidium perfoliatum $L$.

- spinosum $\boldsymbol{L}$.

Lepidonema chilense Fisch., .Mey.

Lepidophorum repandum $D C$.

Lepigonum australe $\boldsymbol{F}$. Mueller.

- canadense Fisch., Mey.

- Iaxiflor tam Barth.

- macrotiecum Fisch., Mey.

- sperguloides Fisch, Mey.

Leptochloa domingensis Link.

Leptosiphon densiflorus Benth.

Lepturus incurvatus Trin.

- subulatus Kitnth.

Lepyrodiclis bolosteoides Fisch.,

Leucas Schimperi Hochst. Hey.

- . urticaefolia $R$. Br.

- velutina $\boldsymbol{C}$. A. Mey.

Leucanthemum atratum $D C$.

- pallens $D^{\circ} C$.

Leuceria senecioides Hook., Arn.

Liatris spicata $\boldsymbol{W}$.

Libanotis condensata Fisch., Mey.

- sibirica C. A. Mey.

Ligularia sibirica $D C$.

Ligusticum alatum $S p r$.

- scoticum $\boldsymbol{L}$.

- Seguieri Koch.

Limnanthemum geminatum Griseb.

Limnanthes Douglasii $R . B r$.

Linaria aequitriloba $\hat{S}$ pr.

- aparinoides Chav.

- canadensis Dum. Cours.

- chalepensis Mill.

- cirrbosa $W$.

- glutinosa Hoffm., Link.

- origanifolia $D C$.

- Pelisseriana Mill.

- praetermissa Delatre

- triornithophora $W$.

Lindenbergia urticaefolia $\mathrm{Lehm}$.

Lindefolia spectabilis Lehm.

Linum monogynum Forst.

isch., Loasa tricolor Lindl.

Mey. - triloba Juss.

Lobelia ocimoides Kunze.

- ramosa Benth.

- trialata Hamilt.

Lolinm Boucheanum Eunth.

- linicola Sond.

- speciosum Stev.

Lomatocarum alpinum Fisch., IIey.

Longchampia capillifolia $\boldsymbol{w}$.

Lophoclinum hirtum Hort.

- Manglesii Endl.

Lotus albidus Lodd.

- conimbricensis Brot.

- ornithopodioides $\boldsymbol{L}$.

- peregrinus $\boldsymbol{L}$.

- pilosissimus Poir.

Lupinus Blaschkeanus Fisch, Mey.

- hirsutissimus Benth.

- nanus Dougl.

- nootkatensis Don.

- perennis $\boldsymbol{L}$.

- polyphyllus Iindl. 
Madia racemosa Torr, Gray. sloltata Fisch, Mey.

Malachua aguaticun Fries.

Maiva abs ssinica $A l, B r$.

- peruviana $\boldsymbol{L}$.

- tricuspidata $\Delta i t$.

Marsypianthus hyptoides Mart.

Martynia lutea Lindl.

Maruta Micheliana Fisch, Mey. - microcephala Schrenk.

Matricaria confnsa Fisch, Mey.

- decipiens C. A. Mey.

- disciformis $D C$

- discoidea DC.

- nigellifolia $\boldsymbol{D C}$

Matthiola parviflora $\boldsymbol{R} . \boldsymbol{B r}$.

Mazus rugosus. Lour.

Medicago retıculata Benth. - secundiflora Dierieu. - striata Bast.

Melampodium divaricatum $D C$. - longifolium Cav.

Melandryum brachypetalum Fenzl. - songaricum Schrenk.

Melica caffrorum Schrzgd.

Melilotus Bonplandi Ten.

- gracilis $\boldsymbol{D C}$.

- messanensis Desf.

- suaveolens Ledb.

- sulcata Desf.

Meniocus linifolius $\boldsymbol{D C}$.

Mentzelia Wrightii A. Gray.

Mercurialis violaefolia Kunze.

Microlonchus Clusii Spach.

- Delilianus Spach.

- elatus Spach.

- tenellus Spach.

Micropus californicus Fisch., Mey.

Microsciadium tenuifolium Boiss.

Mitella diphylla $\boldsymbol{L}$.

Moehringia pentandra Gay.

Mollugo Schrankii Ser.

- verticillata $\boldsymbol{L}$.

Molopospermum cicutarium $D C$.

Monarda aristata Nutt.

Möenchia mantica Bartl.

- quaternella Ehrh.

Monocosmia corrigioloides Fenzl.

Monolepis trifida Schrad.

Monolopia glabrata Fisch., Mey.

- major $D C$.

Monopsis litigiosa Fisch., Mey.

Moscharia pinnatifida $\boldsymbol{R}$. et $\boldsymbol{P a v}$.

Moschosma polystachya Benth.

Myagrum perfoliatum $\boldsymbol{L}$.

Myogalum Boucheanum Kunth. - nutans Link.

Myosotis californica Fisch, Mey.

Myrica Faya Ait.

Nanañthea perpusilla $D C$.

Nasturtium barbareaefolium DC.

-(Sisymbrium Dels)

- clandestinum $S p r$.

- erectum Trev.

- indicum $D C$.

Navarettia pusilla $\boldsymbol{H}$. Paris.

Nemesia floribunda $\mathrm{Lehm}$.

- versicolor $\boldsymbol{E}$. Mey.

Nemophila atomaria Fisch., Mey.

- $\quad-\quad-\beta$ dis-

- aurita Lindl.

- insignis Benth.

- linifiora Fisch, Mey.

- maculata Benth.

- parvifiora Dougl.

Nepeta azurea $\boldsymbol{R} . \boldsymbol{B r}$.

- botryoides Ait.

- camphorata Boiss.

- grandiflora $\boldsymbol{M}$. $\boldsymbol{B}$.
Nepeta nuda $\boldsymbol{L}$.

Neslia apiculata Fisch, Mey.

Nicotiana acuminata $\mathbf{A g h}$.

- alata $L k$., Otto.

- californica Fisch., Mey.

- commutata Fisch., Mey.

- Langsdorffii Wein.. .

- macrophylla $s p r$.

- micrantha $\boldsymbol{H}$. Paris.

- paniculata $\boldsymbol{L}$.

- plumbaginifolia Viv.

- vincaeflora Lag.

Nolana prostrata $\boldsymbol{L}$. fil.

Nonnea alba $D C$.

- nigricans $D C$

- rosea Fisch., Mey.

- versicolor Fisch, Mèy.

Nothoscordum fragrans Kunth.

- striatellum Kunth.

- striatum Kunth.

Notoceras canariense $\boldsymbol{R} . \boldsymbol{B r}$.

Nuttallia malvaeflora Fisch., Trautv.

Ochthodium aegyptiacum $D C$.

Ocimum carnosum Link., Otto.

- graveolens $\mathbf{A l}$. Br.

- micranthum $\boldsymbol{W}$.

Octadenia lybica $\boldsymbol{R}$. $\boldsymbol{B r}$.

Oenothera chilensis $\boldsymbol{H}$. Paris.

- cognata Fisch., Mey.

- concinna Don.

- linearifolia C. A. Mey.

- linifolia Nutt.

- longiflora $\boldsymbol{L}$.

- macrosiphon Fisch., Mey.

- rhizocarpa Spr.

- Romanzovii Ledb.

- roseo-alba Bernh.

- stricta Ledb.

- tenella Cav.

- villosa Thb.

Oldenlandia corymbosa $\boldsymbol{L}$.

Oligomeris glaucescens $C a m b$.

Onobrychis Crista - galli Lum.

Ononis biflora Desf.

- mitissima $\boldsymbol{L}$. -

- órnithopodioides $\boldsymbol{L}$.

- reclinata $L$.

- serrata Forsl.

- sicula Guss.

Oreomyrrhis eriopoda Endl.

Ormenis mixta $D C$.

Ornithogalum Ecklonii Fisch., Mey.

- Szovitsianum Fisch., Mey.

Orobus lathyroides $\boldsymbol{L}$.

- saxatilis Vent.

- tuberosus $L$. $\gamma$ tenuifolius.

Ostericum pratense $\boldsymbol{H} \circ \mathrm{fl}$.

Oxalis micrantha Bert.

- rosea Jacq.

- stricta $I$

- villosa $M . B$.

Oxyria elatior $\boldsymbol{R}$. $\boldsymbol{B r}$ :

Oxytropis candicans $D C$.

- glabra DC.

Paeonia albiflora Pall.

- anomala $\boldsymbol{L}$.

tenuifolia $\boldsymbol{L}$.

Palafoxia texana $\boldsymbol{D C}$.

Panicum capillare Gron.

- erucaeforme Sbth., Sm.

- macrochaetum Link. zonale Guss.

Papaver alpinum $L$. albiflorum.

-. - - flavifloram

amoenum Lindl.

argemonoides Cesati.

- caucasicum $\boldsymbol{M}$. $\boldsymbol{B}$.

- croceum $L e d b$.

- gariepinum Burch.

- horridum $D C$.
Paparer orientale $L$.

- pilosum Sibth., Sm.

Pardanthus chinensis Ker.

Parietaria alsinefolia Del.

- micrantha Ledb.

Paronychia bonariensis $D C$.

Paspalum dilatatum Poir.

Passiflora alba Link, Otto.

- minima $L$

Patrinia sibirica Juss.

Peltaria alliacea $\boldsymbol{L}$.

Pennisetum cenchroides Rich.

Pentameris airoides Nees. ab Es.

Peperomia urocarpa Fisch., Mey.

Peplis alternifolia $M$. B.

- biflora Salzm.

Petiveria tetrandra Gomes. (P. hexaglochin Fisch., Mey.)

Petunia longiflora Mey., Rupr.

Phacocapnos Cracca Bernh.

Phagnalon rupestre $D C$

Phalacrodiscus arvensis Fisch., Mey.

Phalaris angusta Nees ab Es.

- coerulescens Desf.

- paradoxa $\boldsymbol{L}$.

Pharnaceum dichotomum $L$. $\beta$ fili-

Phleum alpinum $\boldsymbol{L}$.

Phlomis alpina Pall.

- tuberosa $L$.

Phyllanthus obovatus Muehlenb.

Physalis lancifolia Nees. ab Es.

- pubescens $\boldsymbol{L}$.

Physocaulos nodosus Koch.

Phyteuma betonicaefolium Vill.

- campanuloides $\boldsymbol{M}$. $\boldsymbol{B}$.

- limonifolium Sibth., $S m$.

- Scheuchzerii All.

Phytolacca purpurea $\mathrm{Al}$. Br., Bonché.

- violacea C.A. Mey. (Brucelia Hort.)

Pimpinella gracilis Bisch.

- rotundifolia $M . B$.

Pircunia esculenta $C$. A. Mey. (Phytolacca Vän Hout.)

Plagiobotrium rufescens Fisch., Mey

Plantago canescens Schrad.

- Durrillei Del.

- Hookeriana Fïsch., Mey.

- Loefflingii $L$. $\beta$ caspia.

- montana Lam.

- tenuiflora W.

- tumida Link.

Platycapnos spicatus Bernh.

Platystigma lineare Benth.

Plectrantbus parviflorus $\boldsymbol{W}$.

Plectritis congesta $D C$.

Plumbago micrantha Ledb.

Poa persica-Trin.

- (Glyceria) songarica Schrenk.

Podolepis aurea C. A. Mey.

- chrysantha Endl.

- gracilis Grah.

Podospermum Jacquinianum Koch.

Polanisia graveolens Rafin.

Polemonium acutiflorum $W$.

Polybotrya incisa Kaulf.

Polycalymna conglutinata $F$. Mueller.

Polygonum acetosum $\mathbf{M B}$.

- herniarioides Del.

Polypogon maritimus $\boldsymbol{W}$.

Portulaca rostellata Brign.

- Thelussonii Lindl.

Potentilla approximata $B g e$.

- chrysantha Trev.

- Jealbata Bge.

- desertorum Bge.

- geoides $\boldsymbol{M}$. $\boldsymbol{B}$.

- kurdica Boiss.

- negalodonton Lehm.

Poteatilla nivea $\boldsymbol{L}$.

- rivalis Nutt.

- stolonifera Lehm.

- strigosa Pall.

- thuringiaca Bernh

- umbrosa Stev.

- villosa Pall.

Prenanthes purpurea $\boldsymbol{L}$.

Primula scotica Hook.

- sibirica Jacq.

Psephelus calocephalus Cass.

Psidium Araça Raddi.

Psilurus nardoides Trin.

Pteroneurum graecnm $D C$.

Pteropogon chilense. Fisch., Mey,

Pterostegiadrymarioides Fisch, Mey.

Pterotheca biflda Fisch, Mey.

- nemausensis Cass.

Ptychotis heterophylla Foch.

Pulegium micranthum Claus.

Pyrethrum ambiguum Ledb.

- Balsamita $\boldsymbol{W}$.

- caucasicum $\boldsymbol{W}$

Pyrrhopappus carolinianus DC.

Quamoclit coccinea Moench.

Ranunculus nodiflorus $\boldsymbol{L}$.

- ophioglossifolius Vill.

- parviflorus $L_{\text {. }}$

- Schraderianus Fisch. Mey.

- trachycarpus Fisch., Mey.

- trilobus Desf.

Rhagadiolus Hedypnois Fisch., Mey.

Rhagudia Preissii Moq.-Tand.

Rheum palmatum $\boldsymbol{L}$.

- undulatum $\boldsymbol{L}$.

Rhodanthe Manglesii Lindl.

Rhodiola atropurpurea Fisch., H $^{\prime} y$.

Rhynchopsidium sessilifiorum DC.

Richardsonia scabra $I$.

Ricotia Lunaria $\boldsymbol{D C}$.

Rochelia Jeiocarpa Ledb.

Roemeria hybrida $D C$.

Rostellaria abyssinica $A l . B r$.

Rulingia corylifolia Grah.

Rumex alpinus $\boldsymbol{L}$.

- brasiliensis $\operatorname{Link}$.

- Brownianus Campd.

- bucephalophorus $L$.

- confertus $\boldsymbol{W}$.

- Gmelini Turcs.

- Marschallianus Rehb.

- parviflorus Fisch., Mey.

- pratensis M., Koch.

- pulcher $\boldsymbol{L}$. 


\section{Semina selecta, e collectione anni 18523 , quae hortus botanicus Imperialis Petropolitanus pro mutua commutatione offert:-}

Ara the theintha Fisch.

Acalj pha alopocuroiden Jacq.

- brakistachya Hornem.

Acanchocephalus amplexifolius Kar.

A anthoceptalus amplexifolius Kar., - arvensis Scop.

Canthosprinum humile $D C$.

Acer tataricum $\boldsymbol{L}$.

L. billen atrata $\boldsymbol{L}$.

- Clavennae $L$.

Gerberi $\boldsymbol{W}$.

leptophylla $\mathbf{M B}$.

mongolica Fisch.

mosiliata Jacq.

-.- speciosa Henk.

Achyrarheen mollis Schaver.

Achyranthes argentea Lam.

A.hirophorus belveticus Scop.

Aconos alpions pers.

- gravolens C.A. Mey.

- origanifolius Rupr. rotmiliroliug Pers.

A and tuberculata Moq. Tand.

Aconitum fimelini Rchb. betonst Rehb.

A. thomeris helianthoides $D C$.

A t poratshurica Turcz. Kir. - fissa-Schum. et Wimm.

- pubescens $M B$. $^{*}$

Allionia albida Wult.

- cucallata Fisch., Mey.

Allium bymenorhizum Ledb.

- obliquam $L$.

- paradorum Fisch.

Alnus fruticusa Rupr.

Alopecuru s agrestis $\boldsymbol{L}$.

- utricalatus Pers.

Alsine arabica Fisch., Mey.

- austriaca Mert., Koch.

- campestris Fenzl.

- globelosa C.A.Mey.

- lari folia Wahlenb.

- pharaaceoides Fenzl.

- rubella Wahlenb.

- Schrenkiana C. A. Mey.

- stricta Wahlenb.

Alternanthers Achyrantha $\boldsymbol{R}$. Br.

- paroajchioides St. Hil.

- sessuta R. Br.

tortuphes denticulata Fisch. var. Althaea Gibulia Cav.

Adertaia maticata $D C$.

Atnmis cirthes Rafn.

A Jome-cautata Stev.

- flamtiea Jacq. var. anomala. stiuar tosa Stev.

$\rightarrow$ Fermatis $I$ latifolia. - hirseta $L$.

- taurzaensis $D C$.

Alyssum crapestre $L$. hirsutum.

- confertum H. Berol.:

- mirsathum C.A. Mey.

- rostritum Stev.

- Szow ianum Fisch., Mey.

- umb itun Desv.

- Wierabickii Heuff.

- Oval. $t$.

- speltwides Tausch.

- squarrosa I., Schreb.

- triartslata Willd.

- friumelalis L., Willt. ventricona Tausch.

A.ropt 'inow alpestre Ledeb.

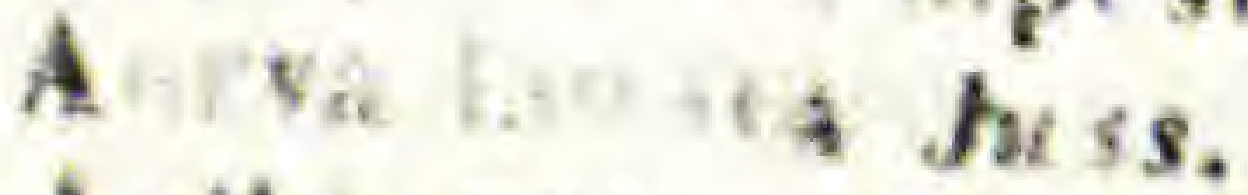

Aetheretu. Muxbanmii $\boldsymbol{D C}$.

- hetcyocarpum Gay.

- saxatite $R . B r$.

Agasy hlis caurasica Spr.

A athosrit oricolises Serbdt.

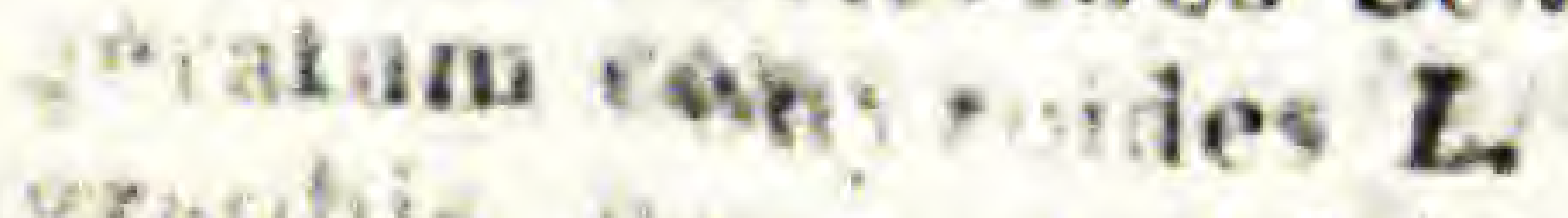

Agrogitio son molata

$\rightarrow$ autas a Rerb.

Agrimenis wffra E. Mey.

- lewswaha hurze.

- pilos ladb.

- rogankt

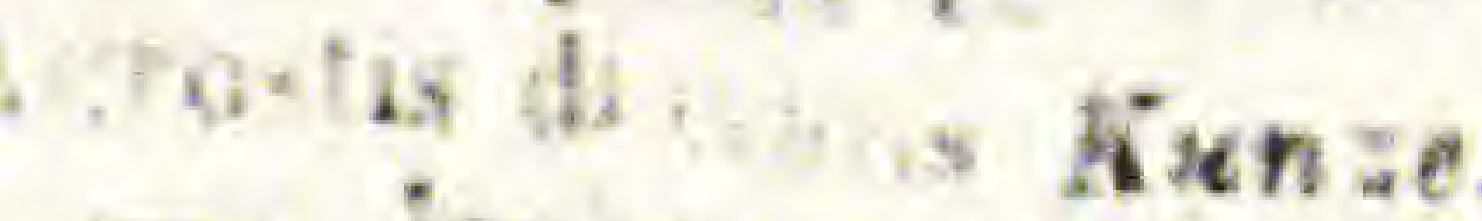

tac aves the v. ab Es.

nistictionss.

palle the

retrum seta wibl.

Ainguforith devata Bsise

Aira capter os sost.
Amarantus abus $L$.

- $\quad$ - $\quad \beta$ parvifforus

- angutifolius $M B$.

- cauítzus $L$.

- hybulus $L$.

- hypeshoudriacus $\boldsymbol{L}$.

- $\quad-\quad \beta$ racemosus

- melancholicus $L . \beta$ tricolor $\boldsymbol{L}$.

- panisulatus $I_{0} \cdot \boldsymbol{\gamma}$ sanguineus $\boldsymbol{L}$.

- parideusis Schk.

-. " retzdfexus $L$.

Rehh. var. - spinasus L.
stellata. - sylvatiois Desf.

Amberbua spoil DC.

- mussuia DC.

Amblyo catzu n iutloides Fisch, Mey.

Ambly oz a polygonoides Rafn.

Ambrosia ritida $\boldsymbol{l}$.

A mellus assuus $\boldsymbol{W}$.

- * - var. tridactylns

Anethyste coeviea $\boldsymbol{L}$.

A momasa if forra $W$,

- by paser Finch.

- isiverata
Ammannia sanguinolenta $S w$. Aquilegia elata Ledb.

\begin{tabular}{|c|c|c} 
- Wormskioldii Hort. Berol. & - fragrans Benth. \\
Amsinckia angustifolia Lehm. & - glandulosa Fisch.
\end{tabular}

- intermedia Fisch., Mey. - - pubilora Wallich."

- lycopsioides Lehm.

- spectabilis Fisch.,_.Mey.

Anacyclus purpurascens $D C$.

- Pyrethrum DC.

- radiatus Lois.

- tomentosus $D C$.

Anarrhinum bellidifolium $W$.

- dnriminium Chav.

Anchusa angustifolia $\boldsymbol{L}$.

- capensis Thbg.

- crispa Viv.

- hybrida Ten.

- leptophylla Röm., Schult.

- Milleri $w$.

- ochrolcuca $\boldsymbol{M B}$.

A ncistrocarpus maypureasis $\mathrm{Humb}$.

Andrographis echioides $\boldsymbol{N}$. ab Es.

Androsace carnea $L$.

$\gamma$ angusta.

- elongata $L$.

- filiformis Retz.

- lactiflora Fisch. grandillora.

A adryala cheiranthifolia Herit.

- integrifolia $L$.

- laxillora $D C$.

Anemone narcíssiflora $L$.

- virginiana $L$.

Angianthus filifolius C. A. Mey.

Anisoderis foetida Cass.

- rhoeadifolia Fisch., Mey.

- rubra Cass.

Anisolotus anthylloides Bernh.

- Wrangellianus Bernh.

Anoda rristata Schlechtd.

- hastata Cav.

- Anthemis altissima $L$.

- austriaca Jacq.

- chia $L$.

- chrysantha Gay.

- granalensis Boiss.

- incrassata Loisl.

- maritima $\boldsymbol{L}$.

- nobilis $L$.

- $\quad \beta$ discoidea.

- peregrina $L$.

- ruthenica $\boldsymbol{M B}$.

- Triumfetti dil.

- Wiedemannia Fisch., Mey.

Anthoxanthum amarum Brot.

- ovatum Lag.

Anthriscus tenerrima Boiss.

- torquata Duby.

Antbyllis cornicina $\boldsymbol{L}$.

- tetrapbylla $L$. $D C$ A aychia capillacea $D C$,

Aphanostephus arkansanus A. Gray.

- ramosissimus $D C$.

Aquitegia atraia Kock.

- atropuryarea $\$$ : Fl chenama

Arabis alpina $L$.

$\div$ stenopelala $w$.
- Skinneri Hook.

- ambigua $D C$.

- auriculata Lam.

- bellidifolia Jacq.

- cebennensis $D C$.

- collina Ten.

- declinata Schrad.

- heteromalla Schrad.

- hirsuta Scop. var. glaberrima

- lilacina Schrad.

- ludoviciana Fisch., Mey.

- rosea $D C$.

- stricta Huds.

- Turrita L.

- verna $R$. Br.

Archangelica officinalis $\boldsymbol{H} \circ / \mathrm{m}$.

- var. disco-
carpa

Arenaria conimbricensis Brot.

- emargizata Brot,

- norregica Gunn.

- spathulata Desf.

Argemone albillora Horn.

- mexicana $L$.

- ochroleuca Siceet.

Argyrolobium velatinum Eckl.,Zeyhi.

Armeria pinifolia Röm., Schult.

- planlaginea $\boldsymbol{W}$. $\varepsilon$. leucantha

- Welwitsebii $D C$.

Boiss.

Arnica Chamissonis Less.

- montana $L$.

Arnoseris pusilla Gaertn.

Aronicum allaicum $D C$.

Arthrolobium ebracteatum $D C$.

- repandum $D C$.

Artemisia borealis Pall. $\%$. Worm.

- desertorum Spr.

skioldi.

Asclepias curassavica $L$. fl. albo.

- incaraata $L$.

Asparagus caspius $\boldsymbol{H}$. Find.

- tenuifolius Lan.

A sprella major Fres.

Aster alpinus $\boldsymbol{L}$.

- Amellus $\boldsymbol{L}$.

- hevis $L$.

- salsuginosus Riehards.

- sibiricus $\boldsymbol{L}$.

Asterolinum adoense $\mathrm{Kz}$.

- stellatum Iink.

Astragalus ad uryens Pull.

- Aegiceras W.

- baeticua $\mathbf{L}$.

- braehyeeras Isdt.

- Treficavtis Ledo. 
Astragalus contortuplicalus $L$.

- cymbaecarpis Brot.

- epiglottis $\boldsymbol{L}$.

- hamosus $L$.

- juvenalis Del.

- leucophaeus Sm.

- pentaglottis $\boldsymbol{L}$.

- scorpioides Poutr.

- sesameus $L$.

- sulcatus $\boldsymbol{L}$.

- vliginosus $\boldsymbol{L}$.

Astrantia Biebersteinii Trautv.

- carniolica Jacq.

- helleborifolia Salisb.

- major $\boldsymbol{L}$.

- minor $\boldsymbol{L}$.

Astrocarpus sesamoides $D C$.

Athamantha sicula $\boldsymbol{L}$.

Atriplex sibirica $\boldsymbol{L}$.

Aubrietia Columnae Guss.

- erubescens Griseb.

Axyris amarantoides $L$.

- hybrida $\boldsymbol{L}$.

Baeria chrysostoma Fisch., Mey.

Baltimora recta $\boldsymbol{L}$.

Baptisia australis $R$. $B r$.

Barbarea prostrata Gay.

Barkhausia alpina $D C$

- bellidifolia $\boldsymbol{D C}$.

- setosa $D C$.

Bartonia aurea Lindl.

Basella rubra $L$. $\beta$ virescens Moq.

Begonia Dregei Dietr., Otto.

- hirtella Link.

Bellevalia romana $R c h b$.

Bellis annua $\boldsymbol{L}$.

- integrifolia Mich.

Bellium bellidioides $L$.

Berteroa orbiculata $\boldsymbol{D C}$.

Beta orientalis Roth

Betckea samolifolia $D C$.

Betonica grandillora $\boldsymbol{W}$.

Betula fruticosa $\boldsymbol{P a l l}$.

Bidens frondosa $L$.

- grandiflora Balb.

- parviflora $\cdot \boldsymbol{W}$.

Bifora radians $\boldsymbol{M B}$.

- testiculata Spr.

Bil̄bergia zebrina Lindl.

Biotia macrophylla $D C$.

Biscutella apula $\boldsymbol{L}$.

- auriculata $\boldsymbol{L}$

- ciliata $\boldsymbol{D C}$.

- erigerifolia $D C$.

- lyrata $L$.

Blainvillea rhomboidea Cass.

Blumenhachia insignis Schrad.

Boissiera bromoides Hochst.

Bonplandia geminiflora Cav.

Borrayo laxiflora $D C$.

Bossiaea ensata Sieb.

- linophylla $\cdot \boldsymbol{R}$. $\boldsymbol{B r}$.

- naucifolia Benth.

- thymifolia Hort. angl.

Botryadenia Gmelini Fisch., Mey.

Botryanthus odorus Funth. ruthe-

- Szovitsii C.A. Mey.

nicus. Capnophyllum africanum Koch.

Bowlesia tenera $S p r$.

Brachycome calocarpa $\boldsymbol{F}$. Miiller.

- diversifolia Fisch., Mey.

- iberidifolia Benth.

Braya siliquosa Bunge.

Briza minor $L$.

Bromus adôensis Hochst.

- brachystachys Hornem.

- brizaeformis Fisch., Mey.

- confertus MB. spicul. mino-

- confertus MB. spicul. pubesc.

- lanuginosus Poir.

- maximus Guss.

- oxyodon Schrenk.
- caucasica Stev.

- rigidus Roth.

- rubens $L$.

- squarrosus $\boldsymbol{L}$.

- vestitus Schrad.

Broteroa trinervata Pers.

Buffonia macrosperma Gay.

Bulbine semibarbata $\boldsymbol{R}$. Br.

Bulliarda trichotoma Eckl., Zeyh.

Bupleurum Gerardi Jacq.

- protractum Link.

- rotundifolium $\boldsymbol{L}$.

- semicompositum $L$.

Cacalia hastata $\boldsymbol{L}$.

Cajophora lateritia Benth.

Calamintha annua Schrenk.

Calandrinia Menziesii $\boldsymbol{H}$ ook.

- micrantha Schlechtd.

- pilosiuscula $\boldsymbol{D C}$.

- procumbens Moris.

- umbellata $D C$.

Calceolaria perfoliata $L$.

- pinnata $\boldsymbol{L}$.

Calendula aegyptiaca Desf.

- brachyglossa Rupr. (C. bicolor Boiss.)

- cristata Guss.

- microcephala Kralik.

- palaestina Boiss.

- sicula Cyr.

- tripterocarpa Rupr., Tigris

Calimeris biennis Ledeb. platycephala Cass.

Callichroa platyglossa Fisch., Mey.

Calliglossa Douglasii Hook.

Callipeltis Cucullaria Stev.

Callistachys retusa Lodd.

Calotis amplexifolia C. A. Mey.

Camelina microcarpa Andrz.

Campanula barbata $L$.

- carpathica $L$. fil.

- Erinus $L$.

- grandis Fisch., Mey.

- lactillora $M B$. var.

- lamiifolia $\boldsymbol{M B}$.

- latifolia $L . \gamma$ eriocarpa.

- linifolia $\boldsymbol{L} \boldsymbol{a m}$.

- Loefllingíi Brot.

- Lorei Pollini.

- nobilis Lindl.

- p'ssilla Haenke.

- sarmatica $\boldsymbol{K}$ ( $\boldsymbol{T}$.

- Stereni $M B$. altaica.

Capnophyllum africanum

Cardamine hirsuta $\boldsymbol{L}$.

- impatiens $\boldsymbol{L}$.

- parvifiora $\boldsymbol{L}$.

- sylvatica Link.

Cardiospermum Corycodes Funze.

- Halicacabum $L$.

Carduus seminudus $\boldsymbol{i} \boldsymbol{B}$.

- tenuiflorus $S \mathrm{Sm}$.

Carex atrata $\boldsymbol{L}$.

- cyperoides $\boldsymbol{I}$.

- festucacea Schk.

Noë.
Bunias Erucago $\boldsymbol{L}$.

- Asterias Fisch., Mey.

Bromus Janceolatus Roth. spicul.pu- Carex Gmelini Hook., Arn.

$$
\text { - bescenl. - punctata Gond. }
$$

- $\quad$ spicul. sca- - rhynchophysa C.A. Mey. bris.

- rostrata Miihlbg.

- stipata Miihlbg.

Carlina longifolia $\mathrm{R} c h b$.

- racemosus L. spicul. glabris. Carpesium cernuum $\boldsymbol{L}$. - spicul. lanugi- Carthamus tinctorius $L$.

nos. Carum Bulbocastanum Koch.

Catananche coerulea $\boldsymbol{L}$.

- lutea $\boldsymbol{L}$.

Caucalis daucoides $\boldsymbol{L}$.

- - - BibericaFisch., Mey.

- leptophylla $\boldsymbol{L}$.

- muricata $\boldsymbol{H}$. Heidelb.

Caylusia abyssinica Fisch., Mey.

Celastrus Aquifolium Fisch.

Celosia margaritacea $\boldsymbol{L}$.

- trigyna $\boldsymbol{L}$

Celsia Chrysurus Fenzl.

- orientalis $L$.

Cenchrus echinatus $\boldsymbol{L}$.

- spinifex Cav., Link.

Cenia pruinosa $D C$.

- turbinata Pers.

Cenocline globifera $C$. Koch.

Centaurea alba $\boldsymbol{L}$.

- arenaria $\boldsymbol{M B}$.

- cristata Burtlg.

- depressa $\boldsymbol{M B}$.

- diluta Ait.

- eriophora $\boldsymbol{L}$.

- exarata Boiss.

- involucrata Desf.

- Kartscbiana Scop.

- leptacantha Fisch., Mey.

- maculosaLam.

montana $L$. var. fl. amethy-Cleome fugax Schra

- - - d citrina $D C$.

- napifolia $\boldsymbol{L}$.

- nigrescens $\boldsymbol{W}$.

- Steveniana Schult.

- violacea $\boldsymbol{L}$.

Clintonia elegans Dougl.

- - $\beta$ vochinensis - pulchella Lindl.

- orientalis $\boldsymbol{L}$.

- - brachylepis.

- orina Pall.

- psendo-Phrygia C.A.Mey.

- pulchella Ledeb.

- Reichenbachii DC.

- rigidifolia Bess. A.

- semperrirens $L$.

- sicula $L$.

- solstitialis $\boldsymbol{L}$.

- lenuiramea Del.

- tenuisecta Jord.

Centranthus dasycarpus Kunze var. Cot macrosiphon Boiss. acheniis glabris

Cephalaria procera Fisch., Lall.

- tataricá R., Schallt.

- transylvanica Schrad.

Cephalophora aromatica Schrad.

Cerastium alpinum $\boldsymbol{L}$.

- anomalum $\boldsymbol{W}$., Kit.

- Beeringianum Cham.

- campanulatum Vis.

- chloraefolium Fisch., Mey.

- dichotomum $\boldsymbol{L}$. $\gamma$ glandulo-

- furcatum Cham.

- inflatum Link.

- latifolium $\boldsymbol{L}$

- microspermum C. A. Mey.

- nemorale $\boldsymbol{M B}$.

- perfoliatum $L$.

- pilosum Ledeb.

- letrandrum $\mathrm{Sm}$. $\beta$ asturicum

Fisch., Mey.

Chamaepeuce stricta $D C$.

Chardinia xeranthemoides Desf.

Charieis heteropliylla Cass.

- Quinoa W.

- $\quad$ - fl. albo.

cirsium acaule $A l l$.

- esculentum C.A. Mey.

Clarkia elegans Dongl.

.

ea Dougl.

- perfoliata Donn.

lypeola Jonthlaspi $\boldsymbol{L}$.

- mierocarpa Hort

Cnicus benedictas $\boldsymbol{L}$.

Cnidium apioides Spr.

Cochlearia anglica $L$.

- glastifolia $\boldsymbol{L}$.

- macrocarpa Fit.

Coix Lachryma $\boldsymbol{L}$.

Collinsia bartsiaefolia Benth.

- bicolor Benth.

- grandiflora Lindl.

- parviflora Lindl.

- sparsiflora Fisch, Mey.

Collomia coccinea $\mathrm{Lehm}$.

- - grandilora Dougl.

- heterophrliă Hook.

- linearis Nutt.

Colpudium bulbosum Trin.

- univittatum Turcz.

Coniringia orientalis Andrz.

Conrolvulus farinosus $\boldsymbol{L}$.

- Pseudo-siculus Cav.

- siculus $\boldsymbol{L}$.

- tricolor $\boldsymbol{L}$.

- undulatus Cav.

Conyza aegypliaca Ait.

- altaica $D C$.

Gouani

- orthoceras $\boldsymbol{D C}$.

Chaenostoma aethinpicum Benth.

thum Benth.

.

Roxburghii Desf. var. radiatum $D C$.

- gypsophiloides Fisch., Mey.

Conioselinum coenolophioides Turcz.

- planisiliqua Fisch., Mey. 
Coreopsis tinctoria Nutt. Corethrostylis bracteata Endl.

Cornucopiae cucullatum $L$.

Coronilla cretica $L$.

Cortusa Matthioli $\boldsymbol{L}$.

Corydalis angustifolia $D C$.

- bracteata Pers.

- bulbosa $D C$.

- claviculata $D C$.

- glauca Pursh.

- nobilis Pers.

Cosmanthus fimbriatus Nolte.

Comos bipinnatus Cav. $\beta$ exarista- $\begin{gathered}\text { - vestitum Wallich. } \\ \text { Deschampsia juncea Beauv. }\end{gathered}$ tus $D C$.

Cotoneaster Nummularia Fisch., Mey.

Cotula anthemoides $L$

- coronopifolia $L$.

- filifolia Thb.

- pusilla $T h b$.

- sororia $D C$.

Cousinia Hystrix C. A. Mey.

Crambe hispanica $L$.

- juncea $M B$.

- maritima $\boldsymbol{I}$.

Crassula lanceolata Endl.

- pubescens Endl.

(C. diaphana hort.)

- Schimperi Fisch., Mey.

Crataegus nigra $\boldsymbol{W}$., Kit.

- punctata Ait.

- subvillosa Schrad.

- sanguinea Pall.

Cremocephalum cernuum Cass.

Crepis aurea Cass.

- Columnae Fröl.

- hieracioides $W . \beta$ croatica.

- multicaulis Ledb.

- parviliora Desf.

- pulchra $L$.

- sibirica $L$.

Crotalaria parvillora Roth.

Crucianella angustifolia $\boldsymbol{L}$.

- chlorostachya Fisch., Mey.

- patula $L$.

- suaveolens C. A. Mey.

Crypsis schoenoides Lam.

Cryptantha glomerata Lehm.

- microcarpa Fisch., Mey.

Cryptostemma calendulaceum $\boldsymbol{R}$. Br. Doronicum caucasicum MB.

- lusitanicum Welw. (Arctotis acaulis Brot.)

Cuphea miniata A. Brongn.

- procumbens Cass.

- silenoides Nees.

- viscosissima Jacq.

Cyclachaena xanthiifolia Fresen.

Cyclamen neapolitanum Ten.

Cyclanthera pedata Schrad.

Cycloloma platyphyllum Moq.

fora $C_{\text {. }}^{\beta}$ parvi-

Cynoglosșum canescens $\boldsymbol{W}$.

- glochidiatum Wallich.

- lanceolatum Forsk.

Cyperus Pumilio N. ab Es. $\beta$ longi-

Cysticapnos africanus Gaertn.

Cytisus albus Link.

- capitatus $\boldsymbol{L}$.

- ratisbonensis Weinm.

Czackia Liliastrum Andrz.

Dactylaena micrantha Schrad.

Datura ferox $\boldsymbol{L}$.

- laevis Lin. fi.

- Stramonium $\boldsymbol{L}$.

Daucus abyssinicus Fisch., Mey.

- glochidiatus Fisch., Mey.

- montevidensis Link.
Daucus muricatus $L$.

Deckera canescens C. A. Mey.

Deeringia baccata Moq.

Delphinium Ajacis $L$.

- cheilanthum Fisch.

- dasyanthum Kar., Kir.

- grandiflorum $L$.

- bybridum $\boldsymbol{W}$. $y$ velutinum.

- lasiostachyum Fisch., Mey.

- ornatum C. Bouché.

- Staphysagria $\boldsymbol{L}$.

- triste Fisch.

Deschampsia juncea Beauv, var. vil- Echium albicans Lag.

Desmanthus virgatus $W$.

losa.

Dianella atrata Fisch.

Dianthes atrorubens $\mathrm{x} l l$.

- capitatus $D C$.

- cruentus Griseb.

- Cyri Fisch., Mey.

- dentosus Fisch.

- Liboschitzianus Ser.

- petraeus W., Kil.

- polymorphus $\boldsymbol{M B}$.

- Pseudo-Armeria

Diastrophis cristata Fisch., Mey.

Dichrocephala latifolia $\boldsymbol{D C}$.

- sonchifolia $D C$.

Dicliptera resupinata Juss.

Didiscus coeruleus $\mathrm{Hook}$.

Dimetopia isocarpa Bartl.

Dimorphotheca pluvialis Mönch.

Diplophyllum veronicaeforme Stev. Elymus Caput-Medusae $\boldsymbol{L}$.

Diplostephium umbellatum $D C$.

Diplotaxis catholica $D C$.

- erucoides $D C$.

- viminea $D C$.

Dipsacus Gmelini $\boldsymbol{M B}$.

Dischisma clandestinum E. Mey.

Disocactus biformis Lindl.

Dudecatheon integrifolium Mich.

Dulichos sinensis Hort. sem. incar-

- tranquebaricus Jacq. sem. majorib. et minorib.

- unguiculatus Jaeq. var. sem. flavis.

- oblongifolium $D C$. var.

Draha Aizoon Wahlenb.

- alpina $L$.

- arabisans Mich.

- aurea Vahl.

- borealis $\boldsymbol{D C}$.

- caroliniana Walt.

- confusa Ehrh.

- contorta Elirh.

- dasycarpa C. A. Mey.

- hirta $L$.

- - dorrina.

- lactea Ad. $\beta$.

- nemorosa $L$.

- rupestris R. Br.

- scandinavica Lindl.

$-\beta$ corono
pifolia.
Dracocephalum parviflorum
Nutt.

Dracopis amplexicaulis Cass.

Drummondia mitelloides $\boldsymbol{D C}$.

Drymaria villosa Sehlechtd.

Echenais carlinoides Cass.

- Sieversii Fisch., Mey.

Echeveria gibbillora $D C$.

Echinaria capitata Desf.

Echinops exaltatus Schrad.

- sphaerocephalus $\boldsymbol{L}$.

Echinospermum caspitum Fisch.,Mey.

- cristatum Bong.

deflexum Lehm.

- laeve $\boldsymbol{C}$. A. Mey.

- marginatum Lehm.

- patulum Lehm.

- Redowskii Lehm.

Eclipta erecta $L$.

- longifolia Schrad.

- patula Schrad.

- - t thermalis-Burge.

Elensine coracana Pers.

- Figarei Delile.

- indica Gaertn.

- oligostachya Link.

Elisanthe diurniflora Rupr. (Silene Kze.)

- noctiflora Endl.

- ornata Rupr.

(Silene Ait.)

- viscida Rupr.

(Silene $\boldsymbol{H} a b$.)

- viscosa Rupr. (Silene Pers.)

Elsholtzia cristata
Elviria biflora $D C$.

- crinitus Schreb.

- geniculatus Curt.

- Hystrix $L$.

Emex spinosa Campd.

Endoptera aspera $D C$.

Epilobium algidum $M B$.

- alpinum $\boldsymbol{L}$.

- chilense H. Berol.

- Durieui Gay.

- minutum Lindl.

- nepalense James.

- origanifolium Lam.

- - var.

rcpandum Spach.

- aegyptiaca Link.

- ciliaris Link.

- mexicana Link.

- peruviana Trin.

- pilosa $P$. de $B$.

- plumosa Link.

- suaveolens Becker.

Erechtites carduifolia $D C$.

pifolia. - hieracifolia Rafn.

- longifolia Fenzl.

Erigeron alpinus $\boldsymbol{L}$.

- elongatus Ledb.

glabellus Nutt.
Echinospermum consanguineum Erigerar glaucus Ker. Fisch. Mey. - teterophyllus H. Berol.

- Diploloma Schrenk.

- heteracanthum Ledb.

- Karelini Fisch., Mey.

longicaule. Erinus apinus $L$. Mittadenia trilobata $\boldsymbol{H}_{\text {ort }}$.)

- rreades Fisch., Mey.

- pdolicus Bess.

- purpureus Ait.

- sjathulatus Vest.

- sjeciosus $D C$.

- sinulosus Hook.

Elaeagnus parvifolia Soc. hort. Lond. Erophib ninima C. A. Mey.

Enarthrocarpus lyratus $D C$.

Entelea arborescens $\boldsymbol{R}$. Br.

- palustre $L$. albiflorum.

(E. squamatum Nutt.)

Eragrostis abyssinica Link.

- chilensis Nees ab Es.

- Purshii Fiseh., Mey.

- tephrosanthos Schalt.

Eranthemum variabile $R$. $B r$.

- valerianaefolia $\boldsymbol{D C}$.

Eremogone graminifolia Fenzl.

- stenophylla Fisch., Mey.

- armerifolius Turcz.

- asperulus Fisch., Mey.

- divaricatus Mich.

- droebachensis Miall.

Erodium alsiniflorum Del.

- abmarium Del.

- Intrys Bertol.

- cuneatum Viv.

- litoreum Lem.

- nalachoides $\boldsymbol{W}$.

- séaceum Del.

- ensenaefolium Del.

$=$ Erucastrm_arabicum Fiscin., Mey.

- ireanum Koch.

- Billichii Sehimp.

Ervum silense Fisch., Mey.

- Euhenackeri Fisch., Mey.

- Liseleurii.$M B$.

- pibescens $D C$.

Eryngiun amethystinum $L$.

- erniculatum Lam.

- giganteum $\boldsymbol{\text { ИВ }}$.

Erysimun Andrzeiorskianum Bess.

- Profskianum Fisch., Mey.

- sigmbrioides C. A. Mey.

- versicolor Andrz.

Erythrae linearifolia Pers: $\beta$ humlis.

Erythronum Dens-Canis $L$. sibiri-

Eschschotzia californica Cham.

Ethulia nnyzoides $\boldsymbol{L}$.

- Knussii $C$. H. Schultz.

Euchariún concinnum Fisch., Mey.

- gandiflorum Fisch., Mey.

Euclidiuñ syriacum $\boldsymbol{R}$. $\boldsymbol{B r}$.

Eucnide sartonioides Zucc.

Eupatorum ageratoides $\boldsymbol{L}$. fil.

- merostemon Cass.

Euphorba dictyosperma Fisch., Mey.

- exgua $L$.

- geniculata Ortega.

- greca Boiss.

- irpericifolia $\boldsymbol{L}$.

- Lzeascae Spr.

- Lathyris $L$.

- Lifolia C.A. Mey.

- merantha Steph.

- muricata MB.

- prta Jacq.

- piosa $L$.

- piùlifera $L$.

- pneera $\boldsymbol{M B}$.

- ptrococea Brot.

- putiescens Vahl.

- trzonocarpa Fiseh.

- vicata W., Kit.

Euryops jectinata Cass.

Eutoca Viangelliana Fisch, Mey.

Euxolus andatus Moq.

- etarginatus Al. Braun. et Bouché. 
alicia tenella Nece.

estuca delicaiula Lag.

- divaricata Leas.

- geniculata w.

- pectinella Dol.

- procumbens hisnth.

- rigida liunth.

- tenuifiora Schrad

- unioloides Funth.

Florestina pedata Cass.

Forskohlea angustifolia Rets.

- tenacissima $\boldsymbol{L}$.

- viridis Ehbg.

Frankenia pulverulenta $\boldsymbol{L}$.

Fritillaria pallidifora Schrenk.

- ruthenica Wiekstr.

Froelichia gracilis Moq.

- interrupta Moq.

Fumaria agraria $\mathbf{L a g}$.

- alexandrina Ehrenb.

- anatolica Boiss.

- capreolata $\boldsymbol{L}$.

- muralis Sondr.

- parviflora Lam.

Gaillardia aristata Pursh.

Galactites tomentosa Mönch.

Galatella dahurica $D C$.

- punctata DC. var. dracuicu- Hebenstreitia integrifolia $L$.

loides. Hedeoma hispida Pursh.

Galega orientalis $\mathrm{Lam}$.

Galeopsis bifida Bönningh.

- pyrenaica Bartlg.

Galinsogea brachystephana $\boldsymbol{B}$. Berol.

- parviflora Cav.

Galium caudatum Boiss.

- divaricatum Lam.

- intricatum Reut.

- murale $D C$.

- nebulosum Boiss.

- parisiense L.. Kock.

- retrorsum $\boldsymbol{D C}$.

- tenuissimum $\mathbf{M B}$.

- trifidum $\boldsymbol{L}$.

- valantioides $M B$

- verficillatum Danth.

Gamolepis Tagetes $D C$.

Garidella Nigellastrum $\boldsymbol{L}$.

Gastridium australe $P$. $B$.

Gastrolobium leptostachyum Hort.

Gatyona globulifera Cass.

Gaudinia fragilis $\boldsymbol{P}$. $\boldsymbol{B}$.

- geminiflora Gay.

Gaultheria Shallon Pursh.

Gaura parviflora Hook.

Gelasia villosa Ctiss.

Genista canariensis $\boldsymbol{L}$.

- candicans $L$.

- chrysobotrys Fisch.

- radiata Scop.

Gentiana pannonica Scop.

- seplèmfida Päll.

- utriculosa $\boldsymbol{L}$.

Geranium aconitifolium Herit.

- affine Ledb.

- albiflorum Ledb.

- carolinianim $\boldsymbol{L}$.

- $\quad$ - petalis pur-

- coeruleum Patr.

- ibericum Cav.

- Londesii Fisch.

- longipes $\boldsymbol{D C}$.

- maculatum $\boldsymbol{L}$.

- nodosum $\boldsymbol{L}$.

- phaeum Lam.

- platypetalum Fisch., Mey.

- Richardsoni Fisch., Trautr.

- sibiricum $\boldsymbol{L}$.

Geu:n brachypetalum Ser.

Geum pallidum Fisch, Mey.

- rubellum $\boldsymbol{H}$. Petr.

- strictum Ait.

- virginianum $\boldsymbol{L}$.

Gilia capitata Dougl.

- laciniata R., Pav.

- nis efoliata Fisch., Mey.

tricolor Benth.

Glossocomia clematidea Schrenk.

Glycosmis citrifolia Lindl.

Gnaphalium cephaloideum $\boldsymbol{W}$.

- pennsylvanicum $\boldsymbol{W}$.

- resedifolium Trev.

- undulatum $\boldsymbol{L}$.

Goldbachia tetragona Ledb.

Gomphrena decumbens Jacq.

Guizotia oleifera DC.

Grangea maderaspatana Poir.

Gratiola latifolia $\boldsymbol{R}$. Br.

Grindelia inuloides $\boldsymbol{W}$.

- squarrosa Dunal.

Gymnococca drupacea $C$. A. Mey.

Gynandropsis muricata Schrad.

Gypsophila acutifolia Fisch.

- elegans $\boldsymbol{M B}$.

- visco:a Murr.

Hasselquistia cordata Jacq.

Hedypnois persica Fisch.

- tubaeformis Ten.

Hedysarum elongatum Fisch.

- Dexuosum $\boldsymbol{L}$.

- spinosissimum $\boldsymbol{L}$.

Helenium autumnale $\boldsymbol{L}$.

- quadridentatum Labill.

Helianthemum denticulatum Thib.

- guttatum Mill.

- intermedium Thib.

- lasiocarpum H. Paris.

- niloticum Pers.

- salicifolium Pers.

- tauricum Rupr.

- villosum Thib.

Helichrysum bicolor Lindl.

- bracteatum $\boldsymbol{W}$.

Heliophila amplexicaulis $\boldsymbol{L}$. fil.

- crithmifolia $\boldsymbol{w}$ :

- integrifolia $L$.

- pusilla $\boldsymbol{L}$.

Heliophytum parviforum $D C$.

Heliopsis buphthalmoides Dun.

Helleborus foetidus $\boldsymbol{L}$.

Helminthia echioides Gärtn.

- stricta Sonder. var. angnsti-

Helosciadium crassipes Koch. $\beta$ he-

- laciniatum $\boldsymbol{L}$.

- leptophyllum $D C$,

Hemerocalfis graminea Andr.

Heracleum asperum Fisc .

- barlatum Ledb.

- iranatense Boiss.

- figusticifolium $\boldsymbol{M B}$.

- palmatifidum Fisch., Lall.

- persícum $\boldsymbol{H}$. Paris.

- pubescens MB.

- villosum Hoffm.

Wilhelmsii Fisch., Mey. .

Hermannia arabica Hochst, Steud

- discolor Otto, Dietr.

Herniaris diandra Bge.

- odorata Andrz.

Heterospermum pinnatur. Cav.

Heuchera americana $\boldsymbol{L}$.

- cylindrica Dougl.

- hispida Pursh.

- pilosissima H. Petr.

Juncus arcticus $\boldsymbol{y}$

Jurinea alata Cass.

Jussieua erecta $\boldsymbol{I}$.

- montana $D C$.

- orientalis $\boldsymbol{L}$.

- saligna L.
Heuchera Richardsonii $R . B r$. Lagascea mollis Cav.

Hibiscus Manihot $\boldsymbol{L}$. var. speciosus. Lagoecia cuminoides $\boldsymbol{L}$.

- ternatus Cav.

Hippocrepis ciliata $D C$.

- unisiliquosa $\boldsymbol{L}$.

Lagurus ovatus $\boldsymbol{L}$.

Hohenackeria bupleurifolia Fisch., Lamatata Fisch., Mey.

Hordeum jubatum $\boldsymbol{L}$.

- marilimum With.

pratense Huds.

Horminum pyrenaicum $\boldsymbol{L}$.

Hutchinsia petraea $\boldsymbol{R}$. $\mathrm{Br}$.

Hymenocarpus nummularius $W$.

Hymenoxis californica Hook.

Hyoscyamus albus $L$.

- canariensis Ker.

- pusillus $\boldsymbol{L}$.

Hyoseris micrucephala Cass.

Hypecoum grandiflorum Benth.

- procumbens $L$.

Hypericum Ascyron $L$.

- orientale $\boldsymbol{L}$.

Hypoxis decumbens $\boldsymbol{L}$.

Jasione perenuis Lam.

Jasonia tuberosa $D C$.

Iberis collina Jord.

- linifolia $\boldsymbol{L}$.

- saxatilis $\boldsymbol{L}$.

- Timeroyi Jord.

Ilysanthes cubensis Fisch., Mey.

Impatiens glandulifera Royle.

Imperatoria caucasica Stev.

- Ostrutium $\boldsymbol{L}$.

Inula caspia Blum. var.

- graveolens Desf.

- hirta $L$.

- Oculus-Christi $L$.

- suaveolens Jacq.

Ionopsidium acaule Rchb.

Johrenia dichotoma $D C$.

Iris Güldenstaedtiana Lep. $\beta$ MB.

- balophila Pall.

- moraeoides Ker.

- ruthenica Ait.

Mey. Lamium bifidum Cyr.

Lantana Geroldiana Otto., Dietr.

Lapsana intermedia $\boldsymbol{M}$. $\boldsymbol{B}$.

Laserpitium gallicum C. Banh.

- latifolium L. a glabrum.

Lasiagrostis Calamagrostic Lin

Lasiospermum pedunculare Lag

Lasthenia Bridgesii Pöpp., L

Lathyrus angulatus $\boldsymbol{L}$.

- Aphaca $L$.

- Cicera $L$

- inconspicuus $\boldsymbol{L}$.

- latifolius $\boldsymbol{L}$. var.

- Nissolia $L$.

- pisiformis $\boldsymbol{L}$.

- pseudo-Aphaca Boiss.

- rotundifolius $w$.

- sphaericus Retz.

tuberosus $L$.

Laurentia Michelii $D C$.

- tenella $D C$.

Lavatera neapolitana $T e n$.

- plebeja Sims.

Lembosema tomentosum C. A. Mey. (Cytisus).

Leontice altaica Pall.

Leonurus glaucescens $B$ ge.

- sibiricus $\boldsymbol{L}$.

tataricus $\boldsymbol{L}$.

Lepidium bonariense $\boldsymbol{L}$.

- campestre $\boldsymbol{R} . \boldsymbol{B r}$.

- Cumingianum Fisch., Mey.

- densiforum Schrad.

- Erkloni Schrad.

- latifolium $\boldsymbol{L}$.

- $\quad-\gamma$ affine Ledb.

- Menziesii $D C$.

- micranthum Ledb. perfoliatum $\boldsymbol{L}$.

IFisch. Lepigonum australe $F$. Mitller.

is campestris Stev. agymnocarpa. - glandulosum Fisch., Mey.

- dasycarpa $\bar{L}_{e} d \dot{b}$.

- intermedia Turcz.

- oblongataStev. $\alpha$.ymnocarpa.

- $-\beta$ lasiocarpa.

- orientalis $W$. var.

- praecox Kit.

- sibirica Trautv.

- taurica Stev.

solepis Saviana $\boldsymbol{R}$., Schult.

Isopyrum fumarioides $\boldsymbol{L}$.

Kalbfussia Müllerl Schultz.

Kennedya rubicunda Vent.

Kentrophyllnm leucocaulon $D C$.

- tauricum Fisch., Mey.

Kernera saxatilis Rchb.

Knautia hybrida Coult.

Koelpinia linearis Pall.

Krubera leptophylla $\boldsymbol{H o f f m}$.

Krynitzkia Teiocarpa Fisch., Mey.

Lablab leucocarpos Savi.

Lactuca altaica Fisch., Mey.

- augustana All.

- cracoviensis Savicz.

- Scariola L. var.

- macrothecum Fisch., Mey.

- medium Fries.

- segetale Fisch., Mey.

- sperguloides Fisch., Mey.

Leptochloa arabica Funth.

- domingensis Link.

Leptopoda brachypoda Torr., Grny

Leptosiphon androsaceus Benth.

Lepturus incurvatus Trin.

- subulatus Kunth.

Lessertia linearis $D C$.

- perennans $D C$.

Leucanthemum murcicum_Gay.:

- pallens $D C$.

Leucas aspera Spreng.

- martinicensis $\boldsymbol{R}$. Br.

- Schimperi Hochst.

- urticaefolia $\boldsymbol{R} . \boldsymbol{B r}$.

Leuceria senecioides Hook., Arn.

Leucojum aestivum $\boldsymbol{L}$. 
Linaria aparinoides Chav.

- canadensis Dum.-Cours.

- chalepensis Mill.

- cirrhosa $W$.

- glutinosa Hoffm., Link.

- litoralis $W$.

- - pterosperma $\boldsymbol{H}$.

- macropoda Boiss., Reut.

- melanantha Boiss., Reut.

- nivea Boiss., Reut.

- origanifolia $D C$.

- Pelisseriana Mill.

- supina Desf.

- triornithophora $\boldsymbol{W}$.

- triphylla $W$.

Lindenbergia urticaefolia Lehm.

Lindheimera texana Gray.

Lin.m angustifolium Huds.

- Loreyi Jord.

- monogynum Forst.

Loasa tricolor Lindl.

- triloba Juss.

Lobelia inflata $\boldsymbol{L}$.

- ocimoides Kunze.

- urens $\boldsymbol{L}$.

Loefflingia hispanica $\boldsymbol{L}$.

Lolium linicola Sond.

Lomatocarum alpinum Fisch., Mey.

Lonas inodora Gaertn.

Longchampia capillifolia $w$.

Lonicera alpigena $L$.

- nigra $L$.

Lopezia racemosa .Cav.

Lotus angustissimus $\boldsymbol{L}$.

- conimbricensis Brot.

- filicaulis Durieu.

- ornithopodioides $\boldsymbol{L}$.

- pilosissimus Poir.

Lunaria biennis Mönch.

Lupinus albus $\boldsymbol{L}$.

- angustifolius $\boldsymbol{L}$.

- Blasclikianus Fisch., Mey.

- mieranthus Dougl.

- nanus Dougl.

- nootkatensis Don.

- perennis $L$.

- polyphyllus $L i n d l$.

Luzula erecta Desv.

- nivea $D C$.

- pediformis $\boldsymbol{D C}$.

Lychnis alpina $L$.

- macrocarpa Boiss., Reut.

- sibirica $L$.

Lycopsis variegata $L$.

Lyperia violacea Benth.

Lythrum tribracteatum Salzm.

Macrorhynchus aurantiacus Fisch.,

- laevigatus Fisch., Mey.

- pterocarpus Fisch., Mey.

Madia racemosa Torr., Gray.

- stellata Fisch., Mey.

- viscosa Cav.

Mahonia Aquifolium Pursh.

Malach um aquaiticum Fries.

Malcolmia bicolor Boiss.

- chia DC.

- lara DC.

- litorea R. Br.

- maritima $R$. Br.

- parvidera $D C$.

- stenopetala Bernh.

Malope trifida Cav.

Malra abyssinica Al. Br.

- Duriaei Spach.

- limensis $\boldsymbol{L}$.

\begin{tabular}{l|l} 
pulchella Bernh. & Möhringia muscosa $L$. \\
Marianthuscoeruleo-punctatus Link, & - pentandra Gay.
\end{tabular}

Marrubium apulum Ten.

Maruta fuscata $\boldsymbol{D C}$.

- discoidea $D C$.

- praecox DC.

Mazus rugosus Lour.

- arenaria Ten.

- ciliaris $W$.

- coronata Lam.

- Echinus $D C$.

- glutinosa $M B$.

- laciniata All.

- laevis Desf.

- lappacea Lam.

- naculata $W$.

- obscura Retz.

- radiata $L$.

- striata Bast.

Melica altissima $\boldsymbol{L}$.

- ciliata $L$.

- gracilis $D C$.

- ruthenica $\overline{M B}$.

- sulcata Desf.

- taurica Ser.

- tricolor $\boldsymbol{W}$.

- elatus Spach.

- erectus $\boldsymbol{L}$.

- supinus $\boldsymbol{L}$.

Milium vernale $\boldsymbol{M B}$.
Marrubium astracanicum Jacq.

- persicum C.A. Mey.

Marsypianthes hyptoides Mart.

- Micheliana Fisch., Mey.

- microcephala Schrenk.

Matthiola tricuspidata $R$. Br.

Maurandia Barcklayana Lindl.

Medicago apiculata $W$.

- disciformis $D C$.

- -Gerardi, $W_{-}$Kit.

- orbicularis All.

- pentacycla $D C$.

- reticulata Benth.

- secundiflora Durieu.

- tribuloides Lam.

- tuberculata W:

Melampodium divaricatum $D C$.

- longifolium Cav.

- rhomhoideum $D C$.

Melandryum brachypetalum Fenzl.

- songaricum Schrenk.

- sylvestre Roehl. 3 Wiedemannianum Lall.

Melanthera deltoidea Rich.

- caffrorum Schrad.

Melilotus Bonplandi Ten.

- messanensis Desf.

- parviflora Desf.

- suaveolens Ledb.

Mentzelia Wrightii A. Gray.

Mercurialis violaefolia Kunze.

Meser.brianthemum cordifolium $L$. Nycterinia selaginoides Benth.

- pinnatifidum $L$. fl.

Metabasis aetnensis $D C$.

Microlonchus Clusii Spach.

- tenellus Spach.

Micromeria bonariensis Fisch., Mey. Odontarrhena tortuosa C. A. Mey.

Micropus ealifornicus Fisch., Mey.

Microsciadium tenuifolium Eoiss.

Mimulus cardinalis Lindl.

- loribundus Lindl.

- luteus $L$. var. quinquevul-

Modiola caroliniana Don.

- - - $\quad$ - Cumingiana. Otto, K1. Mönchia mantica Bartlg.
Molopospermum cicutarium $D C$.

Monarda fistulosa $\boldsymbol{L}$.

Monocosmia corrigioloides Fenzl.

Monolepis trifida Schrad.

Monolopia glabrata $\alpha$ Fisch., Mey.

Monopsis litigiosa Fisch., Mey.

Morina elegans Fisch., Läll:-

Moscharia pinnatifida R., Pav.

Moschosma polystachya Benth.

Myosotis azorica $\boldsymbol{H}$. C. Wats.

- sylvatica Ehrל. $\beta$ alpestris hit.

Myriogyne minuta Less.

Myrrhis odorata Scop.

Nananthea perpusilla $D C$.

Nasturtium barbareaefolium $D C$.

- Camelinae Fisch., Mey.

- clandestinum $S p r$.

- erectum Trev.

- indicum $D C$.

- pyrenaicum $R$. Br.

Navarettia pusilla $\boldsymbol{H}$. Par.

Nemesiá chamaedryfolia Vent.

- floribunda Lehm.

- versicolor $\boldsymbol{E}$. $\boldsymbol{H}$.

Nemophila atomaria Fisch., $\mathbf{M e y}$.

- aurita Lindl.

- insignis Benth.

- liniflora Fisch., Mey. (N. cramboides hort.)

- maculata Benth.

- parvillora Dougl.

Nepeta azurea $R$. $B r$.

- botryoides Ait.

- camphorata-Boiss.

- grandiflor.

Vicotiana acuminata Grah.

- alata Lk., Otto.

- auricnlata Bert.

- californica Fisch., Mey.

- chinensis Fisch.

- commutata Fisch., Mey.

- graciliflora Al. Draun.

- Langsdorfii Weinm.

- micrantha H. Paris.

- paniculata $L$.

- plumbaginifolia Viv.

- vincaeflora $\mathbf{L a g}$.

Nolana prostrata $L$. fil.

Nonea alba $D C$.

- versicolor Fisch., Mey.

fi. Obeliscaria peduncularis Torr., Gray.

Ochthodium aegyptiacum $D C$.

Ocimum canum Sims.

- carnosum Link., Otto.

- graveolens Al. Braun.

- micranthum $\boldsymbol{W}$.

Octadenia lybica $R$. Br.

Oenothera biennis $L$.

- brachypetala Fisch., Mey.

- cognata Fisch,, Mey.

- concinna Don.

- densillora Lindl.

- gracilis Sehrad.

- Lindleyi Dougl.

- longillora Jacq.

- mollissima $L$.

- muricata $\boldsymbol{L}$.

- parviflora L. var.

- pumila $L$.

- purpurea Curt. var.

- quadrivulnera Dougl.

- Romanzowii Ledb.
- nigricans $B C$.

- chilensis $\boldsymbol{H}$. Paris.

Oenothera rosea Ait.

- roseo-alba Bernh.

- sinuata Mich.

- tenella Cav.

- tetraptera Cav.

Oldenlandia corymbosa $L$.

Oligomeris glaucescens $\mathrm{Cam}$,

Oncorhynchus tenellus Fiset, Hey.
Ononis alopeuroides $\mathrm{L}$.

- biflora Desf.

- mitissima $L$.

- ornithopodioides $L$.

- reclinata $L$.

- serrata Forsk. (euphrasiaefolia)

- sicula Guss.

Onopordon acaule $L$.

Opercularia paleata Young.

Orlaya platycarpa Koch.

Ormenis mixta $D C$.

Ornithogalum refractum $\mathrm{K}$.

Ornithopus compressus $L$.

- sativus Brot. (Serradella).

Orobus lathyroides $\boldsymbol{L}$.

- luteus $L$. $\beta$ orientali.

- tuberosus $L$. $\beta$ tenut

Otocblamys Eckluniana $D t$, pin-

Oxalis Bridgesii Bert.

- Dillenii Jacq.

- micrantha Hort.

- Nirbelii Denh.

- rosea Jacq.

- stricta $L$.

- villosa $M B$.

Oxybaphus viscosus Herit.

Oxylobium ellipticum $\boldsymbol{R}$. $\boldsymbol{B}$,

Oxyria elatior $R$. $B r$.

Oxytropis candicans $D C$.

- deflexa $D C$.

- glabra $D C$.

- songaria $D C$.

Paeonia albillora Pall.

- anomala $L$.

- humilis Retz.

- paradoxa Anders.

- peregrina Mill.

- tenuifolia $\boldsymbol{L}$.

Palafoxia texana $D C$.

Pailenis spinosa Cass.

Panicum capillare Gron.

- ciliare Retz.

- eriogonum Schrad.

- erucaeforme Sibth.,

- glabrum Gaud.

- macrochaetum Linh

- Zenkowskii Rupr.

- zonale Guss.

Paparer alpinum L. $\alpha$ alisf rum

- - - B flanherom.

- Argemone $\boldsymbol{L}$.

- caucasicum $\boldsymbol{M B}$.

- croceum Ledb.

- horridum $D C$.

- orientale $\boldsymbol{L}$. 
Paresychia echinata Lam.

Rartbenium affine Fisch., Mey. - Hysierophorus $\boldsymbol{L}$.

Paspalum stuloniferum Bosc.

Passiflora alata sit.

- gracilis Jacq.

Pastinaca Fleiscbmanni Hladn.

Patrinia heterophylla Bge.

Pavonia hastata Cav.

Pelargonium australe $\boldsymbol{w}$.

- humifusum $\boldsymbol{W}$.

Peltaria alliacea $\boldsymbol{L}$.

Pennisetum cenchroides Rich.

Pentastemon ovatus Hook.

- procerus Grah.

- Richardsoni Dougl.

Peplis biflora Salzm.

Petunia Atkinsoniana Sweet.

- longiffora Mey., Rupr.

- nyctaginiflora Juss.

- violacea Lindl.

Peucedanum austriacum Koch.

- Oreoselinum Mönch.

- rablense Koch.

Phaca frigida $\boldsymbol{L}$.

Phacelia circinnata Jacq.

- congesta Hook.

- tanacelifolia Benth.

Phacocapnos Cracca Bernh.

Phagnalon rupestre Cass.

Phalacrodiscus arvensis Fisch.

Phalaris angusta Nees.

- brachystachya Link.

- coerulescens Desf.

- minor $L$.

- paradoxa $L$.

Pharbites hederacea Chois.

- hispida Chois.

Pharnaceum dichotomum $L$. $\beta$ fili- Polycarena capensis Benth.

folium. Polycarpaea latifolia Poir.

Phaseolus compressus $D C$. $\alpha$.

- gonospermos Savi.

- haemitocarpus Savi.

- inamoenus $\boldsymbol{L}$.

- Mungo $\boldsymbol{L r}$

Phleùm annuum $M B^{\circ}$.

- arenarium $\boldsymbol{L}$.

- asperum Vill.

- tenue Schrad.

Phlomis tuberosa $\boldsymbol{L}$.

Phlox Drummondi Hook.

Physalis aequata Jacq. fil.

- lancifolia $\boldsymbol{N}$. ab Es.

- pubescens $\boldsymbol{L}$.

Pbysocaulos nodosus Koch.

Physolepidium repens Schrenk $\beta$.

Phyteuma betonicaefolium Vill.

- campanuloides $\boldsymbol{M B}$.

- limonifolium Sibth., Sm.

- Michelii All.

- orbiculare $\boldsymbol{L}$.

- - Scheuchzerii All.

Phytolacca purpurascens Al. Bi:

- violacea C.A. Mey.

Bouché.

Picridium arabicum Hochst., Steud.

- hispanicum Poir.

- tingitanum Desf.

- vulgare Desf.

Picris pauciflora $\boldsymbol{W}$.

- Sprengeriana Lam.

- squarrosa Steez.

Pilea muscosa Lindl.

- robusta Hort. Hafn.

Pimpinella rotundifolia $\boldsymbol{M B}$.

- Tragium vill.

Pinardia anisocephala Cass.

Piptatherum multiflorum $\boldsymbol{P}$, de $\boldsymbol{B}$.

Pircunia esculenta C. A. Mey.

- Coronopus $\boldsymbol{L}$.

- Cynops $L$.

Gouani Gmel.

- Lagopus $\boldsymbol{L}$.

- mexicana Link.

- saxatilis $M B$.

- tenuiflora $W_{\bullet}$, Kit.

- truncata Cham. virginica $L$.

- triangulare $\boldsymbol{R} . \boldsymbol{B r}$.

Plectritis congesta $\boldsymbol{D C}$.

Plumbago mirantha Ledb.

Poa persica Trin.

Pocockia cretica Ser.

Podolepis aurea C. A. Mey.

- chrysantha Endl.

- gracilis Grah.

Polanisia graveoles $\mathrm{Hook}$.

Polemonium humile $\boldsymbol{W}$.

- mexicanum Cerv. (conglutinata).

- Bellardi All. var.

- herniarioides Del.

- salignum $\boldsymbol{W}$.

Polypogon maritimus $w$.

- monspeliensis Desf.

- rostellata Brign.

- arguta Pursh.

- canescens Bess.

- gariepensis Eckl.

- glandulosa Lindl.

- gracilis Dougl.

- Hippeana Lehm.

- birta $L$.

- kurdica Boiss.

- multifida $\boldsymbol{L}$.

- rivalis $N u t t$.

- rupestris $\boldsymbol{L}$.

- stolonifera Lehm.

- supina $L$.

- taurica- $\boldsymbol{W}$.

- umbrosa Stev.

- villosa Purs!.. है!

Delortii Jord.
Plagiobothrium rufescens Fich., Poterium stenolophum Jord.

Plantago amplexicanlis $C a v$. ${ }^{\prime} y$. Prenanthes purpurea $\boldsymbol{L}$.

- arenaria $\boldsymbol{W}_{\text {., Kit. }}$. gargarea. - uudulata Fisch.

- canescens Schrad. Psilonema calycinum C. A. Mey.

Psilurus nardoides Trin.

Psoralea corylifolia $\boldsymbol{L}$.

- Hookeriana Fisch., Mey.

- Loefflingii $\boldsymbol{L}$. $\beta$ caspia.

- tumida Link. $\beta$ califoraea. Pterotheca bifida Fisch., Mey.

Platylobium parviflorum $S \mathrm{~m}$.

Platystemon calıfornicum Benti

Plectranthus parviflorus $\boldsymbol{W}$.

Psychine stylosa Desf.

Pleroneurum graecum $D C$.

Pteropogon chile..se Fisch., Mey.

- - Fisch., Mey.

Pterostegia drymarioides Fisch.,

Podospermum Jacquinianum Koh.

Podotheca angustifolia Less.

- trachysperma Torr., Gray

Polycalymna Stuartii Mïll., Sot.

Polycarpon alsinefolium $D C$.

Polycenia hebenstreitioides Choit.

Polygonum argyrocoleum Steud.

- mexicanus Steud. (non $S_{p}$.)

(P. sessiliflorus Rupr.)

Portulaca maculata Engelm.

- montevidensis C.A. Mey.

Potentilla approximata Bge.

- chrysantha Trev. var.

- digitato-flabellata $A l$. Br.

Bouclo

Ptychotis heterophylla Koch.

Pulegium micranthum Claus.

Pulicaria arabica Cass.

Pulsatilla albana $S p r . \beta$.

Pultenaea tenuifolia $\boldsymbol{R}$. Br.

Puschkinia scilloides $\boldsymbol{A d}$.

Pyrethrum ambiguum Ledb.

Pyrrhopappus carolinianus $\boldsymbol{D C}$.

Pyrus baceata $L$. $\alpha$ praecox.

Quamoclit coccinea Mönch.

- hyperboreus Rottb.

- undata $\boldsymbol{L}$. $\therefore$

- megalodonton Lehm.

- pulcherrima Lehm.

- recta $L$. $\gamma$ laciniosa Koch.

Poterium agrimonoides $L$.

- polygamum W., Kit.
Mey.

- nemausensis Cass.

- verna Mill.

- Balsamita $\boldsymbol{W}$.

- carneum $\boldsymbol{M B}$.

- caucasicum $W$.

- multicaule $\boldsymbol{W}$.

(Coleostephus Durieu).

- myriophyllum C. A.Mey.

- baccata $L$. $\beta$ serotina.

- cerasifera Tausch $\alpha$ xantho-

- - $\quad-\gamma$ macro-

- oblongata C. A. Mey.

carpa.

- $\quad-\quad-\beta$ luteola.

anunculus abortivus $L$.

- lomatocarpus Fisch., Mey.

- millefoliatus Vahl.

- muricatus $\boldsymbol{L}$.

- nodiflorus $\boldsymbol{L}$.

- ophioglossifolius Vill:

- parviflorus $L$.

- pennsylvanicus $\boldsymbol{L}$.

- Philonotis Ehrh.

- Schraderianus Fisch., Mey.

- trachycarpus Fisch., Mey.

- trilobus Desf.

- tuberculatus Kit.

Rapistrum glabrum Host.

- rugosum Berg.

Reseda crystallina $\mathbf{W e b b}$.

- inodora $\boldsymbol{R} \boldsymbol{c h b}$.

- Luteola $\boldsymbol{L}$.

- Phyteuma $L$.

Rudbeckia laciniata $\boldsymbol{L}$.

Rulingia coryifolia Grah.

- dumosa $\boldsymbol{H}$. Petr.

Rumex alpinus $\boldsymbol{L}$.

- Brownianus Campd.

- bucephalophoras $\mathbf{L}$.

- confertus $\boldsymbol{W}$.

- Gmelini Turez. var.

- hamatus Trev.

- maritinus $\boldsymbol{L}$.

- Marschallianus Rchb.

- maximus Schreb.

- parviflorus Fisch., Mey.

- songaricus Sehrenk.

- stenophyllas Ledb. $\beta$.

- ucranicus Fisch.

- verticillatus $L$.

- vesicarius $L$.

Sagina decumbens Ell.

- maritima Don.

- saxatilis Wimm.

- stricta Fries.

Salpiglossis sinuata R., Pav.

Salsola collina Pall.

Salvia argentea $\boldsymbol{L}$.

- clandestina $\boldsymbol{L}$.

- dumetorum Andr.

- hirsuta Jacq.

- hispanica $\boldsymbol{L}$.

- Horminum $L$.

- Kochiana Funze.

- officinalis $\boldsymbol{L}$.

- plebeja $\boldsymbol{R}$. Br.

- rhombifolia R., Pav.

- Sclarea $L$.

- Spielmanni Scop.

- spinosa $\boldsymbol{L}$.

- utifis Al. Braun.

- verticillata $L$.

- $\quad$ - $\delta$ calyce mu

- viridis $\boldsymbol{L}$.

Samolus litoralis $\boldsymbol{R} . \boldsymbol{B r}$.

Sanguisorba alpina Bunge.

- media $\boldsymbol{L}$.

- sitchensis C. A. Mey.

- tenuifolia Fisch. $\alpha$ leucantha. - $\beta$ rbodantha.

Saponaria cerastoides C.A.Mey.

- chloraefolia Eunze.

- porrigens $\boldsymbol{L}$.

Sarracha Jaltomata Schlechtd.

Saxifraga Aizoon $\boldsymbol{L}$. petalis oblongis.

- caespilosa $L$.

- controversa Sternb.

- cordifolia Hav.

- Geum $L$.

- hirsuta $L$.

- irrigua $M B$. -

- muscodes Wulf.

- mutata $\boldsymbol{L}$.

- orientalis Jacq.

- rirularis $L$.

- rotundifolia $L$.

- stellaris $L$.

- tridactylites $L$.

Rhagodia Preissiana Moq.

Rheum crassinervium $\boldsymbol{H}$. Findb.

- sibiricum Pall.

- undulatum $L$. var.

Rhodauthe Manglesii Lindl.

Rhodiola atropurpurea Turcz.

Richardsonia scabra $\boldsymbol{L}$.

Rivina tinctoria Hamilt.

Roberlia tarasacoides $\boldsymbol{D C}$.

Rochelia leiocarpa $\tilde{L}_{e d}$.

- stellulata Rehb.

Rodigia commutata $S$ pr.

Roemeria hybrida $\boldsymbol{D C}$.

Rostellaria abyssinica $A l$. Brann.

Rudbeckia fuigida $\Delta i t$.

Scabiosa atropurpurea $\boldsymbol{L}$.

- caucasica $M B$.

- Fischeri $D C$.

- Hladnickiana Host.

- micrantha Desf. 
Schizanthus lilacinus Kunze. Schizopetalum Walkeri Sims. Schkubria abrotanoides Roth. - senecioides $N$. ab Es.

Schmidtia coronopifolia Cass. Scilla peruviana $\boldsymbol{L}$.

$$
\text { __ verna Huds. }
$$

Scirpus atrovirens $W$.

Scleropus amarantoides Schrad.

Scorzonera taurica $\mathbf{M B}$.

Scrofularia arguta Ait.

- chrysantha Jaub., Spach.

- divaricata Ledb.

- laciniata W., Kit.

- lur.dillora Fisch., Mey.

- orientalis $\boldsymbol{L}$.

- $\quad$ - $\quad$ - ebulifolia $M B$

- peregrina $L$.

- sambucifolia $\boldsymbol{L}$. $\beta$ hirsuta.

- Scopoli Hoppe.

- . smyrnaea Boiss.

- sublyrata Brot.

Scutellaria alpina $\boldsymbol{L}$.

- commutata Guss.

- japonica Morr., Decais.

- polybotrya Bernh.

Secale fragile $\boldsymbol{M B}$.

Securigera Coronilla $D C$.

Sedum Aizoon $\boldsymbol{L}$.

- albescens Haw.

- annuum $\boldsymbol{L}$.

- caespitosum $D C$.

- Cepaea $L . \delta$ heterophyllum.

- cyaneum Rud.

- hispanicum $L$.

(sexfidum $M B$.)

- kamtschaticum Fisch.

- leptorhizum Fisch., Mey.

- multicaule Wallich.

- oppositifolium Sims.

- pallidum $\boldsymbol{M B}$.

- spurium $\mathbf{H B}$.

- stellatum $L$.

Selago fasciculata $\boldsymbol{L}$.

Sempervirum caespitosum $\boldsymbol{C}$. $S m$.

Senebiera Coronopus Poir.

- pinnatifida $D C$.

Senecio arabicus $\boldsymbol{L}$.

- Cineraria DC.

- inaequidens $\boldsymbol{D C}$.

- Iyratifolius Koch.

- macrophyllus $M B$.

- Raoulii Spach.

- subalpinns $\boldsymbol{K}$ och.

- ustulatus $\boldsymbol{D C}$.

- viscosus $\boldsymbol{L}$.

Serjana guaraminea Mart.

Serratula centauroides $\boldsymbol{L}$.

- coronata $\boldsymbol{L}$.

Seseli gracile $\boldsymbol{W}$, Kit.

Sherardia arvensis $\boldsymbol{L}$.

Sibbaldia cuneata Edgevo.

- procumbens $\boldsymbol{L}$.

Sicyos bryoniaefolia Moris.

Sida Abutilon $\boldsymbol{L}$.

- angustifolia Lam.

- rhombifolia $\boldsymbol{L}$.

- spinosa $\boldsymbol{L}$.

- vesicaria Cav.

Sideritis lanata $\boldsymbol{L}$.

- montana $L$.

- romana $I$.

Siegrest rkia doscuiosa Heril.

- nimalayensis H, Borol.

- iherica $\boldsymbol{w}$.

- orientalis $L$.

Silaus carvifolius C. A. Mey.

Silene alpestris Jacg.

- ambigua Camb.
Silene annulata $T$ hore

- antirrhina 1

- aprica Tur.

- Armeria $L$.

- Atocion Mar.

- bellidifolia neq.

- Bergeri Scintt.

- catholica Gis.

- compacta Als.

- cosoidea $L$.

- Deliliana Fisk., Mey.

- disticha $W$.

- fuscata Lint

- hirsuta $\left.L_{2}\right\}$ asturica.

- inamoena Ladl.

- integripetad Bory., Chaub.

- italica DC.

- jurenalis be

- liniçola Wi

- linifolia $W$.

- longicilia Ucr, Welw.

- museipula $L$

- nemoralis $v$, Kit.

- nicaensis Al

- obtusifolia $\%$

- orientalis $H_{v t}$.

- pendula $L$.

- picta Pers.

- praecox Lin?

- pygmaea Liv.

- repens Pat

- rubella $L$.

- saponariaefo Schott.

- saxatilis Sir

- Saxifraga $L$.

- Schafta Gme.

- sedoides Jac

- sericea All.

- tenuiflora Goy.

- velutina Pors.

- vespertiaa frs.

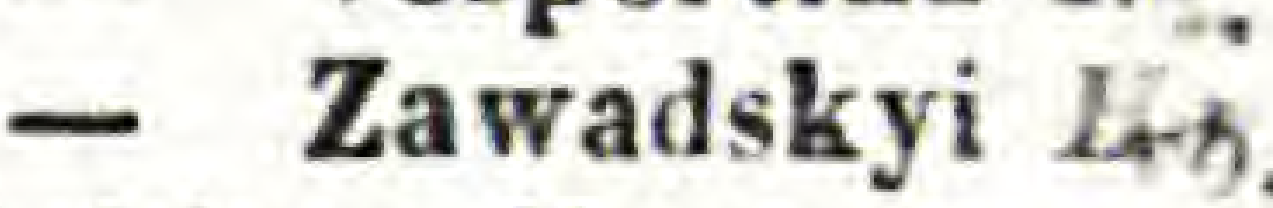

Silphium Hornemas; Schrad.

- perfoliatum

Sinapis hispida Schoob.

Sisymbrium binerves A. Mey.

- brach petaluisch., Mey.

- bursifolium $\mathrm{L}$.

- Columnae dos

- -3.

- Cumingianemzisch., Mey.

- incanum Berf.

- nitidum Zece.

- pumilum SteF.

- subhastatum $f$.

- villosum Lag.

Sium lancifolium $M E$

- medium Fisch rey.

Smelowskia alpina $C_{\text {i }}$ Mey.

- brevicaulis C. Mey.

Sogalgina trilobata $C_{4}$.

Soja hispida Mönch.

Solanum Balbisii Du*

- ciliatum Lam.

- citrullifolium Brawn. (violaceum Engm.

- guineense Lar

- heterodoxum

- laciniatum Ait

- palustre Pöpg.

Soliva anthemifolia $P_{y}$.

- Barklayana $D C$.

- sessilis R., Pat.

Sollya angustifolia Lia:

Sonchus tenerrimus $\mathcal{H}$.

Sorema paradoxa Lizel

Spananthe paniculata.

Specularia billora $\mathrm{Kisi}^{2}$ Yey.
Specularia falcata $A . D C$. - hybrida $A . D C$.

- Pentagonia A. DC.

- perfoliata $A . D C$.

- Speculum A. DC.

Spermacoce tenuigr $L$.

Sphàerolobium medium $R$. Br.

- vimineum $S m$.

Spharostigma Chamissonis Fisch.,
- hirtum Fisch., Mey.

- minutiflorum Fisch., Mey.

Sphallerocarpus Cyminum Bess.

Sphenandra viscosa Benth.

Sphenogyne adonidifolia $D C$.

- anthemoides $\boldsymbol{R} . \mathrm{Br}$.

- microcephala $D C$.

- versicolor $D C$.

Spilanthes fusca $\boldsymbol{H}_{\text {. }}$ Paris.

- leucocephala H. Berol.

- oleracea $L_{0}$ :

- Pseudo-Acmella $L$.

Stachys annua $\boldsymbol{L}$.

- arabica Hornem.

- arvensis $L$.

- menthaefolia Vis.

- setifera C. A. Mey.

Stellaria scapigera $\boldsymbol{L}$.

Stemodia Ehrenbergiana Schlechtd. - parriflora Ait.

Stenopetalum sphaerocarpum $\boldsymbol{F}$.

Stipa tortilis Desf.

Strophiostoma amoenum Rupr.

Succovia balearica Medic.

Sutherlandia frutescens $\boldsymbol{R} . \boldsymbol{B r}$.

Symphyandra pendula $D C$.

Synedrella nodiflora Gaertn.

Talinum patens $\boldsymbol{W}$.

- reflexum Cav.

- teretifolium Pursh.

Taraxacum bicolor $D C$. $\alpha$ subdenta-

- caucasicun DC.

- glaucanthum $D C$.

Tauscheria gymnocarpa Fisch.

- lasiocarpa Fisch.

Telanthera polygonoides Moq. $\beta$ bra-

Teloxys aristata Moq. chiata Schrad.

Tephrosia ochroleuca Pers.

Tetragonia echinata Ait.

- expansa Ait.

Tetragonolobus conjugatus Ser.

Tetrapoma barbareaefolium Turcz.

Teucrium lamiifolium Urv.

- orientale $\boldsymbol{L}$.

Thalictrum dasycarpum Fisch,

- exaltatum C.A. Mey.

Lall.

- globiflorum Ledb.

- kemense Fries.

Thelygonum Cynocrambe $\boldsymbol{L}$.

Thermopsis fabacea $D C$.

Thisantha glomerata Eckl., Zeyh.

- lateriramea Fisch., Mey.

Thlaspi arenarium Jord.

- ceratocarpum Murr.

- Gaudinianum Jord.

- sylvestre Jord.

glabres- Thlipsocarpus baeticus Kunze.

cens. Thomasia discolor Steud.

- purpurea Gay.

Tiaridium indicum Lehm.

Tillaea connata $\boldsymbol{R}$. $\boldsymbol{P}$.

- muscosa $\boldsymbol{L}$.

Titbonia tagetiflora Desf.

Tordylinm apulum $\boldsymbol{L}$. syriacum $L$.

Iordis nodosa Gurtn.

Trachyandra echenandioides $\boldsymbol{H}$. Berol.

Tradescantia erecta Jacq. - undata $W$.

Trianthema monogyna $L$.

Trtchodesma indicum Lehm.

Trichonema ramiflorum Sweet.

Trifolium angustifolium $\boldsymbol{L}$.

- canescens $W$.

- clypeatum $\boldsymbol{L}$.

- diffusum Ehrh.

- erinaceum $\boldsymbol{M B}$.

- filiforme $L ., S m$.

- glomeratum $\boldsymbol{L}$.

- Gussoni Ten.

- hirtum All.

- incarnatum $\boldsymbol{L}$.

- intermedium Guss.

- latinum Sebast.

- leucanthum $\mathbf{M B}$.

- libanoticum Ehrenb.

- ligusticum Balb.

- maritimum Huds. fl. albido.

- Michelianum Savi.

- minus $S m$.

- nidificum Griseb.

- nigrescens Vir.

- ochroleucum $L$.

- pannonicum $\boldsymbol{L}$.

- parviflorum Ehrh.

- procumbens $L$.

- resupinatum $L$.

- Sebastiani Savi.

- spadiceum $\boldsymbol{L}$.

- spumosum $L$.

- squarrosum $L$.

- striatum $L$.

- strictum $L$.

- suaveolens $\boldsymbol{W}$.

- subterraneum $I$.

- suffocatum $L$.

- tomentosum $L$.

- trichocephalum $\boldsymbol{M B}$.

Trigonella calliceras $F i s c h$.

- cancellata Desf.

- coerulea Ser.

- corniculata $L$.

- ensifera Trautv.

- Foenum graecum $\boldsymbol{L}$.

- monantha C. A. Mey.

- monspeliaca $L$.

- ornithopodioides $D C$.

- orthoceras Kar., Kir.

- pinnatifida Cav.

- platycarpa $L$.

- polycerata $\boldsymbol{L}$. 
Trtice Dodarlii $L$.

- elougata Liuk.

- membranacea Poir.

- mexicana $\boldsymbol{H}$. Berot.

- pilulifera $t$.

Vaccinium Arctustaphylos $L$.

Vaillantia hispida $\boldsymbol{L}$.

- muralis $\boldsymbol{L}$.

Valeriana alliariaefolia Vahl.

- montana $L$.

- Phu $L$.

- Iripteris $\mathbf{L}$.

Valerianella Auricula $D C$.

- brachystephana Steud.

- coronata DC. $\beta$ discoidea.

- dentata DC.

- echinata $D C$.

- eriocarpa Desf.

- hanata Bast.

- hirsutissima Link.

- Morisonil DC.
Valerianella oxyrhynchs Fisch., Mey. Vicia Bobarlii Forst.

- plagiostephana Fisch., Mey. - grandidora Scop.

- pumila DC.

- Szovitsiana Fisch., Mey.

- vesicaria Mönch.

Velezia rigida $\mathcal{L}$.

Verbascum pyramidatum $\mathbf{M B}$.

- Wallichianum Benth.

Verbena Aubletia $\boldsymbol{L}$.

- biserrata Kunth.

- urticaefolia $\boldsymbol{L}$.

Veronica anagalloides Guss.

- biloba $L$.

- Cymbalaria Bertol.

- gentianoides Vahl.

- peregrina $\boldsymbol{L}$.

- sibirica $\boldsymbol{L}$.

- urticaefolia $\boldsymbol{L}$.

Vesicaria corymbosa Griseb.

- gracilis Hook.

- sinuata Poir.

- occulta Lehm.
- Michauxii Spr.

- narbonensis $L$.

- pannunica Jacq.

- peregrina $\boldsymbol{L}$.

- picta Fisch., Mey.

- platycarpos Roth.

- Pseudo-Cracea Bertol.

- villosa Roth. $\beta$ glabrescens.

Viola gracilescens Jord.

- vivariensis Jord.

Wahlbergella affinis C. A. Mèy.

- angustiflora Rupr. $\beta$.

- propinqua C. A. Mey.

Wahlenbergia capensis $\mathrm{Alph}$. DC.

- gracilis Alph. DC.

- lobelioides Alph. DC.

- stricta G. Don.
Weigelia Midde odorfuna Traut.

Westriagis lotgifolía $\boldsymbol{R}, \boldsymbol{B r}$.

Wiedenanaia orientalis Fisch., Hey.

Withania somnifera Dun. \& flexuo*a,

Xanthisma texana DC. $\beta$ Druamoos!

Xanthíum orientale $\mathcal{L}$. fil

- riparium Laasch.

Xanthogalum purpurascens I.all

Xeranthemum annuau $\boldsymbol{L}$. - erectum Presl.

- inapertum $\boldsymbol{L}$.

Ximenesia encelioides $\mathrm{Cav}$.

Zazintha verrucosa Gärtn.

Zinnia elegans Jacq.

- multiflora $\boldsymbol{l}$.

- tenuiflora Jaeq.

Ziziphora capitata $\boldsymbol{L}$.

- tenuior $L$.

\section{Semina c reglonibus wolgo-caspilis.}

Asperula humifusa Bess.

Astragalus albicaulis $D C$.

- macropas Bge.

- reticulatus $\mathbf{M B}$.

Corispermum filifolium $C$. A. Arey.

Cousinia volgensis $C$. A. Mey.

Erysimum Andrzeiovskianum Bess. Nasturtium brachycarpum C.A.Mey.

Glaucium corniculatum Curt. $\beta$ na- Ononis intermedia $C$. A. Mey. villorum.
Juncus salsuginosus Turcz. $\beta$ pallens. Polygonum strictum Ledb. Jurinea lnearifolia $D C$. Rumex ucranicus $F$ isch.

Leonurus glaucescens $B$ ge. glaber. Saussurea crassifolia $\boldsymbol{D C}$.

Melilotus ruthenica $\boldsymbol{M B}$.

Microphysa ruhioides C. A. Mey.

Scorzonera ensifulia MB.

Statice caspia $\boldsymbol{W}$.

- latifolia $S m$.

- sareptana Al. Beck.

- sumruticosa $L$.
Statice tomentella Boiss.

Triticum desertorum Fisch. longesubulatum.

- sibiricum $\boldsymbol{W}$.

Tulipa tricolor Ledb.

Vicia picta Fisch., Mey.

\section{Anotationes.}

Aegilops speltoides Tausch. et A. ventricosa Tausch. genuinae (Contra adversar. h. Monac et Vindob.).

Dichrocephala sonchifolia DC. genuina (Contra advers. h. Hamburg.). D. Latifolia h. Hamb. 1832. 3. erat D. soachifolia. Vera D. latifolia DC. 4832 excepta ex hert. Parisino el Christian. specimina transitoria non obtulit.

Eragrostis suaveolens Beck., Claus. Locallor. der Wolgagegend. p. 266, longe diversa est ab E. poaeoide et potius ad E. mexicanam accedit (Contra advers. h. Monac.).

Ilysanthes cubensis $\boldsymbol{H}$. Petr. est genuina generis species; filamenta enim antica in media parte denticulo parvo instructa sunt.

Macrorhynchus laevigatus et pterocarpus in unam speciem conjungi haud potest; utraque longa annorum serie culta characteres servavit. (Conf. Advers, h. Halens.)

Maria stellata H. Petr. "Duas hujus generis species dedil Molina in historia naturali Chilense sativam scilicet foliis petiolatis, et melosam foliis amplexicaulibus, quibus proculdubio radii sunt longissimi juxta laudatum auctorem: diversa ergo ab istis erit haec nostra planta - et $M$. stellata - cujus radii breves et folia sunt sessilia, nullatenus amplexicaulia.n Cavan. Icon. et descript. plant. Vol. III p. 50. In M. stellata folia sunt lineari-lanceolata, sessilia, calathidia pauciflora squamis $\mathbf{5}$ exterioribus longitudine interiorum $\mathbf{5}$ persistentium, corollulae radii parrae, breves.

Oenothera cognata $\boldsymbol{H}$. Petr. ab $\mathbf{O}$. odorata tubi calycis parte superiore elongata statim distinguitur.

Sagina saxatilis Wimm. S. filiformis Pourr. est species haud bene nota; planta in hortis hoc sub nomine culta ad S. strictam Fries pertinet, sed nostra $\mathbf{S}$. saxatilis est species pentamera.

Valerianella pulchella ex h. Berol. 1852. 3. est V. pumila var. tridentata Stev. Valerianella quaedam sub nomine "pulchella », numquam ex h. Petropolit. emissa est. Idem valet de Atriplice polysperma horto Petropolitano adscripta. (Conf. Advers. h. Vindob.)
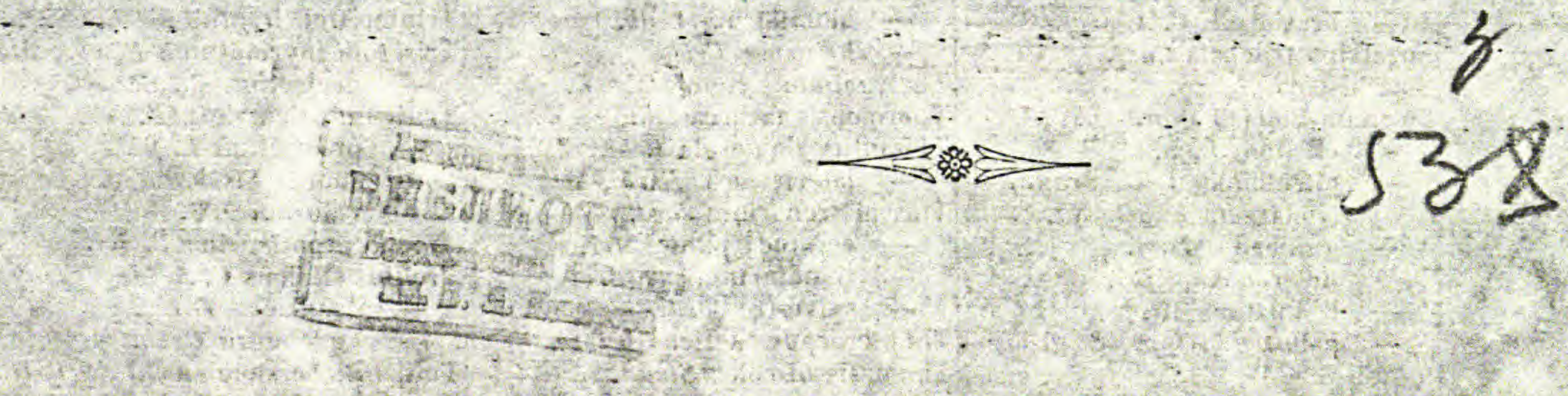

O. A. MEYER,
praelectus borti.

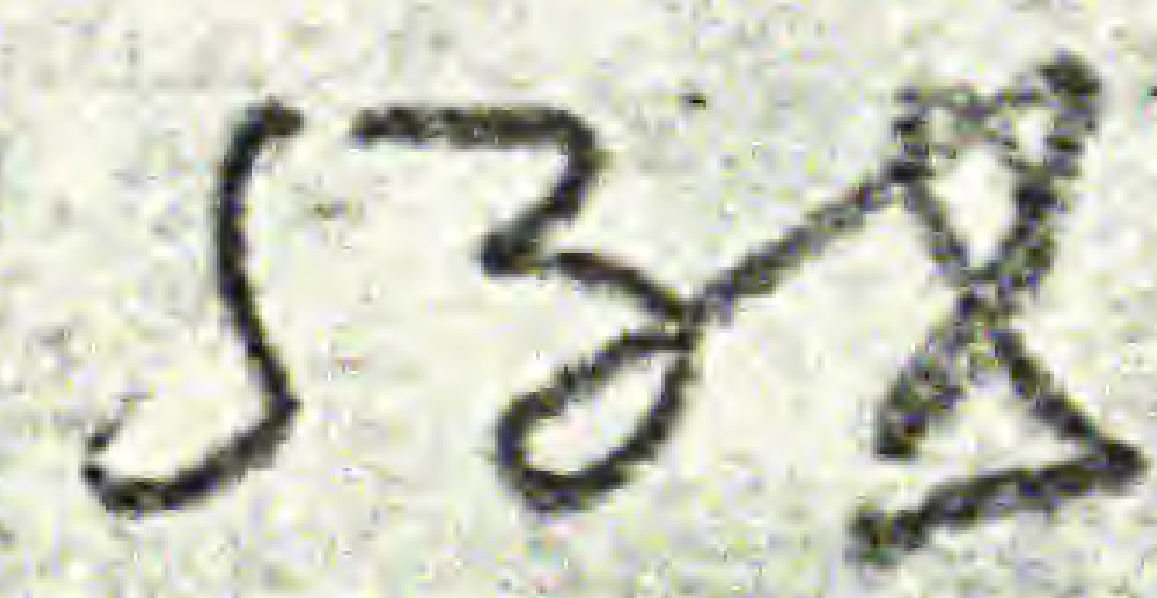

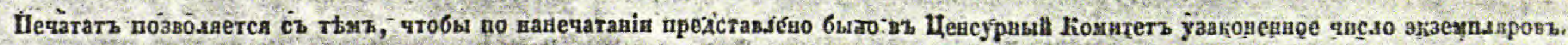

C. Петербургъ, 6 Января 1834.

Цевсорз Ю. ШІи иховски. 


\section{INDEX SEMINUM}

QUAE

\section{HORTUS BOTANICUS IMPERIALIS PETROPOLITANUS}

PHO

\section{MUTUA COMMUUTATIONE OFFERT. :}

Accedunt animadversiones botanicae nonnullae.

$\mathrm{a}=$ = planta aunua. $\mathrm{h} .=$ planta biemis. $\mathrm{p}=$ =planta perennis. $1 .=$ planta lignosa. $\mathrm{fr} .=$ frigidarium. $\mathrm{c} .=$ calidarium. Planta non signatae sub dio crescunt.

\section{6.}

Acacia armata $R$. Br. I. fr. Acalypha alopecuroides Jacq. a. c. Achillea filipendulina Lam.'p.

- Gerberi Wiltd. p.

- lanata Spr. p.

- leptophylla $\boldsymbol{M}$. B. p. Achyrophorus maculatus $\mathcal{S c o p}$. p. - pinnatifidus DC. p. , Aconitum Anthora $L$. p.

- barbatum Patr. p.

3. hispidum

\section{Ledeb. p. \\ - Kusnetzowii Rchb. p.}

- Napellus L. var. (A.) Bernbardianum Rchb. p.

- - var. compactum rubellum Rchb. p.

- - var. (A.) Fúnkianum

Rehb. p.
- $\quad$ var. (A.) laetum Rehb.

- $\quad$ var. (A.) laetum Rchb.
- $\quad$ p.
- var. (A.) pyramidale Mill. p.

- orientale Mill. p.

- variegatum L. var. (A.) nasutum Fisch. p.

Acroclinium roseum Hook. I. fr. Actinospora diahurica Turcz. p.
Adenophora denticulata Fisch. var. latifolia. p.

- marsupiflora Fisch. p.

Adonis caudata Stev. a.

- squarrosa Stev. a.

- vernalis $L$. p.

Aegilops speltoides Tausch. a.

- triaristata Willd. a.

Aerva lanata Juss, a, c.

Aeschynanthus pulcher Steud. I. c.

Aethionema Buxbaumii DC. a.

Agasyllis eaucasica $S p r$. p.

Agathosma imbricata Thby. I. fr.

Agrimonia leucautha Kze. $p$.

- pilosa Ledeb. p.

- procera Wallr. p.

- repens $L$. p.

Agrostis distans $\boldsymbol{K} z e$. a.
- pallida $\boldsymbol{D C}$. a.

- retrofracta Willd. a.

- verticillata Vill. a.

Aira capillaris Host. a.

- Cupaniana Vis. a.

Aizoon canarieuse $L$. a. c.

Alchemilla alpina $\boldsymbol{L}$. p.

- pubescens MB. p.

- vulgaris L. $\beta$. subsericea Koch. p. 
Alfredia cernua Cass. p.

Allium angulosum L. p.

- caeruleum Pall. p.

- $\quad$ - $\gamma$. album H. Petrop. p.

- caesium Schrenk, p.

- chloranthum Lallem. p.

- Cistulosum $L$. p.

- $\quad$ -. .ceratophyllum Bess.p.

- hymenorhizum Ledeb. p.

- Ledebourianum Schult. fil. p.

- obliquum Schrenk. p.

- odorum $\boldsymbol{L}$. p.

- Ophioscorodon Don. p.

- polyphyllum Kar, Kir. p.

- strictum Schrad. p.

- Victorialis $\boldsymbol{L}$. p.

Alonsoa Warscewiczii $\mathrm{Rgl}$. I. fr. Alsine globulosa C. A. Mey. a.

Alstroemeria haenantha $\boldsymbol{R} . \boldsymbol{P}$. p. fr. psiltacina Lehm. var. longipedunculata $\boldsymbol{R} g l$, p. fr.

Alternanthera Achyrantha $\boldsymbol{R}$. Br.a.c.

- ficoidea $R ., S$. a. c.

- spinosa R., $S$. a. c.

Alyssum hirsutum $M B$. a.

- micranthum Fiseh, Mey. a.

- minimum Willd. a.

- petraeum Arduin. b.

- rostratum Stev. b.

- Szovitsianum Fisch., Mey. a.

- umbellatum Desv, a.

A mblogyna polygonoides Rafin. a.c. Amblyocarpum inuloides Fisch., Mey. b.

A mbrosia arlemisiaefolia $L$. a. maritima $\boldsymbol{L}$. a.

Amelanchier Botryapium DC. 1. Ammannia senegalensis Lam. a. c. Amygdalus nana $L$. 1 .

Anacyclus pulcher Bess. a.

- purpurascens Pers. a.

- radiatus Loisl. a.

- tomentosus DC. a.

Anarrhinum bellidifolium Willd. b. A nchusa ochroleuca MB. p.

Andersonia sprengelioides $\boldsymbol{R}$., $\boldsymbol{B r}$. I. fr.

Androsace maxima $\boldsymbol{L}$. a.

Androsaemum officinale $\mathrm{All}$. p.

Andryala cheiranthifolia VHerit. a.

- integrifolia L. a.

- laxiflora $D C$. a.
Anemone multifida Poir. $\gamma$. Hudsoniana. p.

- narcissiflora $L$. p.

- sylvestris $L$. p.

Anona hexapetala $L$. fil. I. c.

Antennaria alpina Gaertn. p.

Anthemis nobilis $L . \alpha$, radiata b.

- $\beta$. discoidea $b$.

Anthephora elegans Schreb. a. c.

Anthoxanthum aristatum Boiss. $\beta$. la-

xun H. Havn. a.

- ovatum Lagasc, a.

Anthurium coriaceum Siveet. l. c.

Anychia dichotoma Michx. a.

A phanostephus arkansanus A. Gray. a. c.

Aquilegia advena If. Petrop. p.

- atropurpurea Willd. p.

- elata Ledeb. p.

- glandulosa Fisch., Mey. p.

- hybrida Sims. p.

- jucunda Fisch., Lallem. p.

- sibirica Lam. p.

- sibirica Lam. ß. stenosepala Rgl. p.

- vulgaris $L$. $\beta$. caucasica $L e$ deb. p.

Arabis hirsuta Scop. b.

- saxatilis All. a.

- verna $R . B r$. a.

Arbutus Andrachne $L$. I. fr.

- Unedo $L$. I. fr.

Archangelica officinalis $\mathrm{Ho} / \mathrm{fm}$.

- - B.discocarpa Lall.p.

A renaria conimbricensis Brot. a.

- emarginata brot. a.

- glomerata MB. a.

- muralis Sieb. a.

- tenuifolia $L, \delta$. brachypetala Ledeb. a.

Armeria plantaginea Willd. $\varepsilon$. leucantha Boiss. p. fr.

- pungens $R ., S$. p. fr.

- Welwitschii Boiss. p. fr.

Arnica Chamissonis Iess. p.

Arthrolobium scorpioides DC. a.

Asparagus caspius Hohenack. p.

Asperugo procumbens $L$. a.

Asperula arvensis $\boldsymbol{L}$. a.

- setosa Jaub., Spach. a.

Asphodelus ramosus $L$. p. 
Aster alpinus $\boldsymbol{L}$. p.

- montanus Richards. p.

Astragalus adsurgens Pall. p.

- Aegiceras Willd, a.

- juvenalis Del. a.

- micranthus Desv. p.

- platyglottis $L_{\text {. }}$ a.

Astrantia Biebersteinii Trautv. p.

- minor L. p.

Axyris amarantoides $L$. a, c.

- hylorida $L$. a. c.

Baptisia australis $R$. Br. p.

Bartonia aurea Lindl. a. c.

Beckmannia erucaeformis Host. p.

Begonia Dregei Otto., Dietr. 1. c.

- hirtella $L k$. a. c.

- nitida Dryand. I. c.

- semperflorens Lk., Otto I. c.

- sinuata Wall. I. c.

Bellevallia comosa Koch. $\beta$. praecox p.

- romana $R c h b$. p.

Betckea samolifolia $D C$, a

Betonica grandillora Stev: p.

Biscutella apula $L$. a.

- auriculata $L$. a.

- erigerifolia $L$. a.

- Jyrata L. a. hispida Rgl. a.

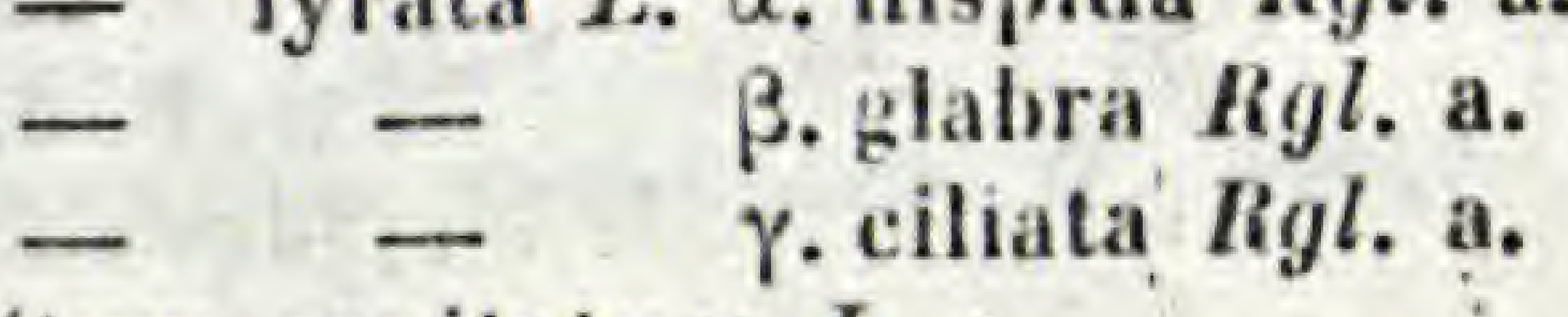

Blitum capitatum $L$. a.

- polymorphum C. A. Méy. a.

- virgatum $L$. a.

Boschniakia glabra C. A. Mey. p.

Bossiaea heterophylla $\mathrm{Sm}$. I. fr.

- linophylla Br. I. fr.

- paucifolia Bth. I. fr.

Brachycome diversilolia Fisch., Mey. p. fr.

Brassica oxyrrhyncha Cass. a. ,

- Schimperi Boiss, a.

- Tournefortii Gouan. a.

Browallia viscosa Humb., Bónpl. a. c.

Bupleurum fruticosum $L$. L. fr.

- Gerardi Jacq. a.

Cakile maritima Seop. a,

Calceolaria glutinosa $R g h$. a. fr?

- lobata Cav. I. fr.

Callicarpa americana $L$. I. fr.

Callipeltis Cucullaria Stev. a.

Callisace dahurica Fisch. p.

Callistachys ovata Sims, 1. fr.
Callistachys retusa Lodd.'I. fr.

Callistemon lanceolatum $D C$, I. fr.

- lineare DC. I. fr. .

- pinifolium DC. I. fr.

- rigidum $R$. Br. I. fr.

- speciosum DC. I. fr.

Callitris australis Sweet. I, frr.

Calyptrostigma Middendorffiana

Trautv., Mey. 1.

Campanula colorata Wall. $\beta$. Moorcroftiana $A, D C$. b. fr.

- Hohenackeri Fisch., Mey. p.

Canna maculata $L k$. p. c.

- patens Rosc. p. c.

- tenuiflora Bouché, p. c.

Capnophyllum africanum Gat. $\mathbf{n}$. a.

Capsella procumbens Fries. a.

Carex caucasica Stev. p. ,

- Ecklonii N. a. E. p. fr.

- leiorhyncha C. A. Mey. p.

Carmichaelia australis $R$. Br. I. fr.

Carrichtera Vellae $D C$. a.

Cassia laevigata Willd. I. c.

Cas ...rina pumila Otto,, Dietr. I. fr.

Catalpa syringaefolia Sims. I. fr.

Caucalis daucoides $L$. $\beta$. iberica Fisch., Mey. a.

- muricata $\boldsymbol{H}$. Hevidelb. a.

Centaurea macrocephala Muss. Pusk. p.

Cerastium glomeratum Thuill. a.

- glutinosum Fries. $\alpha$. obscu-

rum Koch. a.

- grandiflorum $W ., \boldsymbol{K} . \mathbf{p}$.

- illyrioum Ard. a.

- inflatum $L k$. a.

- latifolium L. $\alpha$. latifólium Ledob. p.

- manticum L. a.

- microspermum C. A. Mey. a.

- perfoliatum L. $\alpha$. glaberrimum Rgl. a.

- $\beta$ ciliatum Rgl. a.

Ceratocapnos umbrosa Dur. a. c. Ceratocephalus falcatus Pers. a.

- orthoceras DC. a.

Cercis Siliquastrum $L$. Ivifr.

Chaerophyllum aureum $\boldsymbol{L}$. p.

Chaeturus fasciculatus $L K$. a.

Chamaemelum uniglandulosum Vis.a.

Chamaepeuce Cassabonae DC. b. fr.

- stricta DC. p. fr. 
Chamaerops humilis $\boldsymbol{L}$. I. fr. Chenopodina maritima Moq. a.

Chenopodium panieulatum Hook. a.

Chimonanthus fragrans, Lindl. I. fr.

Chorispora tenella $D C$. a.

Chorizema cordatum Lindl. I. fr.

Chrysanthemum grandiflorum Willd. $\beta$. intermedium $D C$. 1 fr.

- grandiflorum Willd. $\gamma$. subnudum $D C$. I. fr.

Cirsium acaule $\mathrm{All}$. b.

- arachnoideum $\boldsymbol{M B}$. p.

- borridum MB, p.

- munitum $M B$. b.

- setigerum Ledeb. p.

Cleistanthium nepalense Kze. p. fr. Clematis integrifolia $L$. p.

Clethra arborea Ait. I. fr.

Cochlearia anglica $L$, a.

- aretica Sehlechtd. a.

- glastifolia $\boldsymbol{L}$. p.

- macrocarpa $\boldsymbol{W} ., \boldsymbol{K}$. p.

- ofticinalis $\boldsymbol{L}$. b.

- $\quad$ - . minor Rgl. b.

Coelopleurum Gmelini Ledeb. p.

Collomia heterophylla Hook. a.

- stenosiphon Kze. a.

Conioselinum Fischeri Wimm., Grab. - univittatum Turez. p. [p.

Conoclinium janthinum Morr. I. c.

Conringia planisiliqua Fisch., Mey. a.

Convolvulus pseudosiculus $\boldsymbol{C a v}$. a.

- siculus $L$. a.

Conyza ambigua $D C$. a.

Cornucopiae cucullatum $\boldsymbol{L}$. a.

Cornus sericea L'Herit. $\mathbf{L}$.

Cortusa Matthioli $L$. p.

Corydalis claviculata $D C$. a.

$$
\text { - glauea Pursh. a. }
$$

Cotoneaster frigida Wall. L. fr.

- laxiflora Jaeq. 1.

- microphylla Wall. l. fr.

- uniflora Bge. 1.

Crambe hispanica $L$. a.

Crassula diaphana E. Mey. a. C.

- lanceolata Endl. a. c.

- Schimperi Fisch., Mey, a. c.

Crepis sibirica $L$. p.

Crucianella chlorostachys Fisch., Mey. a.

Cryptomeria japonica Don. I. fr. Cucumis prophetarum $L$. a. c.
Cucumis sphaerocarpa Iall. a. c.

Cucurbita aurantiaca Willd, a. c.

- microcarpa Lall. a. c.

- ovifera $L$. a. c.

- verrucosa 1 . a. c.

- $\quad$ - $\beta$. laevis a. c.

Cupressus sempervirens $L$. var (C.) fastigiata $D C$. I. fr.

- sempervirens L. var.(C.) horizontalis Mill. I. fr.

Cuscuta Cephalanthi Engelm. a. Cynoglossum viriditlorum Pall. p.

Cyperus pygmaeus Rottb. a. c.

Cysticapnos africana Gaertn. a. c.

Cytisus albus $L k$. I. fr.

Cytisus chrysobotrys Fisch., Mey. 1. fr.

Czackia Liliastrum Andrz. p.

Dactyloctenium aegyptiacum Willd. a. c.

Daphne altaica Pall. 1 .

Daucus glochidiatus Fisch., Mey. a.

- montevidensis $L k$. a.

- muricatus $\boldsymbol{L}$. a.

Delphinium cardiopetalum DC. a.

- elatum L. a. Ledeb. p.

- - 3. a. subglabrum Ledeb. a.

- $\quad$ - $\quad$ B.b. hispidum Ledeb. p.

ß. c. subciliatum Ledeb. p.

- cheilanthum Fisch. p.

- dasyanthum Kar., Kir. p.

- dictyocarpum DC. p.

- gracile DC. a.

- hybridum Willd. $\beta$. fl. atropurpureis Ledeb. p.

- lasiostachyum Fisch., Mey. p.

- oruatum C. Bouché. a.

- pubescens DC. a.

- triste Fisch. p.

Desmodium canadense $D C$. p.

Deutzia scabra Thbg. I.

Dianthus eapitatus DC. p.

- cruentus Griseb. p.

- Seguieri Vill. $\beta$. Ledeb. p.

- superbus $\boldsymbol{L}$. p.

Digitalis nervosa Steud. p.

Dimelopia hirta Benth. a. c.

- isocarpa Bartl. a. c.

- Walpersii Bge. a. c. 
Diospyros Lotus L. 1. fr. Diplotaxis virgata $D C$, a.

Dipteracanthes Schauerianus $\boldsymbol{N}$. a. E. I. c.

Dischisma capitatum Chois. a. c.

- clandestinum E. Mey. a. c.

Dodecatheon integrifolium Michx.p.

Doronicum caucasicum $M B$. p.

Draba Aizoon Wahlbg. p.

- alpina $\boldsymbol{L}$. p.

- arabisans Michx. b.

- aurea Vahl. b.

- borealis DC. p.

- hirta. L. p.

- incana $L$. p.

- - $\quad$. legitima Lindbl. p.

- lutea Gilib. a.

- minima Steud. a.

- rupestris $R$. $B r$. $\beta$. minor Fisch., Mey. p.

Dracocephalum altaiense Laxm. p.'

- parvillorum Nutt. p.

- nutans $L$. p.

- Ruyschiana L. p.

- thymiflorum $L$. b.

Drummondia mitelloides $D C$, p.

Dyckia remotiflora Otto., Dietr. p.c.

Echeveria gibbinlora DC. I. fr.

Echinocactus corynodes $\boldsymbol{H}$. Berol. I. c.

Echinopsis Eyriesi i Pfeiff., Otto. I. c.

- turbinata Pfei/f., Ottq. I. c. .

- Zuccarinii Pfeiff. I. c.

Echinospermum caspium Fisch.; Mey, a.

- dellexum Lehm. b. .

Echium rubrum Jacq. p.

Elaeocarpus cyaneus Sims. l.' fr.

Ephedra andina Pöpp. I. fr.

Epilobium aftine Bong. a.

- alpinum L. p.

- brevifolium Don. a.

- coloralum Miihlbg. p

- hypericifolium Tausch. p.

- mexicanum DC. a.

- minutum Lindl. a.

- montanum $\boldsymbol{L}$. p.

- $\quad$ - . lanceolatum IT. Paris. p.

- origanifolium Lam. p.

- palustre $L . \alpha$. linearifolium Rgl, p.
Epilobium palustre $L$. $\beta$. virescens Rigl. p.

- parviflorum Schreb. p. [p.

- roseum Schreb. p.

- tetragonum L. p.

- uralense Rupr. a.

- virgatum Fries. p.

Eragrostis abyssinica $L k$, a.

- megastachya Lk. a.

- mexicana $L k$. a.

- plumosa $L k$. a.

- Purshii Schrad, a.

Eremogone longifolia Fenzl. p.

Erica baceans $L$. I. fr.

- concinna Soland. I. fr.

- lateralis Willd. I. fr.

- - transparens Andr. l. fr

Erigeron acris $L$. $\beta$. asteroides $D C$. b.

- $\quad$ - $\gamma$.serotinus Ledeb. b.

- alpinus $\boldsymbol{L}$. p.

- $\quad$ - $\quad$. elatus Hook. p.

- armerifolius Turcz. $\alpha$. humi-

lis Ledeb. b.

- divaricatus Michx. A..

- Droebachiensis Miill. b.

- glabellus Nutt. p.

- glabratus Hoppe. p.

- oreades Fisch., Mey. b.

- spathulatus Vest. a.

- speciosus DC. p.

- spinulosus Hook., Arn. a.

- uniflorus $L$. p.

- Wightii DC. a.

Eritrichium californicum A. DC. a.

- pedunculare $A . D C$. a.

- rupestre Bunge. p.

Erodium Botrys Pers. a.

- chium Willd. a.

- fallax Jord, a.

- gruinum Willd. a.

- litoreum Pers. a.

- malacoides Willd. a.

† verbenaefolium Del. a.

Erpetion reniforme Sweet. p. fr.

Erucaria aleppica Gaertn, a.

Erucastrum arabicum Fisch., Mey. a.

- incanum Koch, a. ha

Ervum chilense Fisch, Mey, a.

- himalayense A.Br, Bouché. a.

- parvillorun Bertol.;a.

- pubeseeus $D C$. a. 
Ervum Terronii Ten. a.

Erysimum Kunzeanum Boiss, a.

- sisymbrioides C. A. Mey. a.

Erythronium dens canis $L$, p.

Eucharidium concinnum Fisch., Mey. a.

- grandiflorum Fisch., Mey. a.

Eudianthe laeta Rchb, a. - oculata Lindl. a.

Eupatorium ageratoides $\boldsymbol{L}$. p. - microstemum Cass. a. - purpureum $\boldsymbol{L}$. p.

Euphorbia falcata $L$. a.

- Lagascae Spr. a.

- latifolia C. d. Mey. p.

- macrorhiza C. A. Mey. p.

- pilosa $L$. p.

- pterococca Brot. a. c.

- pubescens Vahl. a.

- segetalis $\boldsymbol{L}$. a.

- terracina $D C$. a. c.

- trigonocarpa Fisch.p.

- virgata $\boldsymbol{W}$., K. p.

Eurotia ceratoides $C$. A. Mey. a. Falcaria dahurica $D C$. p.

Fedia graciliflora Fisch., Mey, a. Ferulago sylvatica Rchb. b.

Festuca delicatula Lagasc. a.

- geniculata Willd. a.

- pectinella Del. a.

- procumbens $K t h$. a.

- rigida $\boldsymbol{K} t h$. a.

- tenuiflora Schrad. a.

Filago spathulata Presl, a.

Forskohlea viridis Ehrbg. a. c.

Fritillaria pallidiflora Schrenk. p.

- ruthenica Wickstr. p.

Froelichia gracilis Moq. a. c.

- interrupta Moq. a. c.

Fumaria agraria Lag. a.

- alexandrina Ehrbg. a.

- anatolica Boiss. a.

- capreolata $L$. a.

- media Loisl. a.

- micrantha Lag. a.

- muralis Sonder, a.

- parviflora Lam. a.

Galactites Durieui Spach, a. - tomentosa Mönch. a.

Galatella dahurica $\boldsymbol{D C}$. p.

- Hauptii Lindl. $\gamma_{\text {. tenuifolia }}$ Lall. p.
Galega ofleinalis $\boldsymbol{L}$. $\beta$. (G.) africana Mill. p. orieutalis Lam. p.

Galinsogea brachystephana $\boldsymbol{H}$. Berol, a.

Gastridium australe Beauv, a.

Gastrolobium cuneatum A. Henfr. 1. fr.

Gaudinia fragilis Beauv. a.

- geminiflora Gay, a.

Gaya simplex Gaud. p.

Gelasia villosa Cass. p.

Genista canariensis $\boldsymbol{L}$. l. fr.

- ramosissima Webb, l. fr.

Gentiana lutea $L$. p.

- macrophylla Pall. p.

- Pneumonanthe L. p.

- septemfida Pall. p.

Geranium affine Ledeb. p.

- collinum Steph. $\alpha$, glandulosum Ledeb. p.

- $\quad$ - $\beta$. eglandulosum Le-

- phaeum L. p. [deb. p.

- platypetalum Fisch., Mey. p.

- sibiricum L. p.

- viscosissimum Fisch,, Mey.p.

- Vlassovianum Fisch. p.

Geum brachypetalum Ser. p.

- macrophyllum Willd. p.

- pallidum Fisch., Mey. p.

- rubellum Fisch., Mey. p.

- spurium Fisch., Mey. a. urbano-strictum $C, A$. Mey. p.

- $\quad$ - $\beta$. stricto-urbanum $c$. A. Mey. p.

- strictum Ait. p.

- Willdenowii Fisch., Mey. p.

Gilia laciniata $R$, $P$. a.

- millefoliata Fisch., Mey. a.

- multicaulis Benth. a.

Glinus lotoides $L$. $\beta$, virens Fenzl. a. c. Glossocomia clematidea Fisch., Mey. Gloxinia caulescens Lindl. p. c. [p. Gnaphalium purpureum $L$. b.

- resedaefolium Trev, a,

- spicatum Lam. a.

- undulatum $L$. a.

Goldbachia torulosa $D C$. a.

Goodia lotifolia Salisb. I. fr.

Grangea maderaspatana Poir. a. c.

Gratiola latifolia $\boldsymbol{R}$. Br. a. 
Grindelia arguta Sehrad. p. fr. - inuloides Willd. p. fr.

Gynandropsis muricata Schrad. a.c. Gypsophila acutifolia Fisch. p.

- arenaria $K ., W$. p.

- perfoliata $L, \alpha$. angustifolia Fenzl. p.

- repens $L$. p.

Halleria lucida $L$. I. fr.

Hasselquistia cordata $\boldsymbol{L}$. fil. a. Hebenstreitia integrifolia $L$. a. Hedysarum elongatum Fisch. p.

- inundatum Turcz. p.

- neglectum Ledeb. p.

- obscurum L. p.

- sibiricum Poir. p.

Helenium brachyglossum Lall. p.'fr.

- quadridentatum Lab. a.

- teuuifolium Nutt. a.

Helianthemum ledifolium Pers. a. - salicifolium Pers. a.

Helichrysum bicolor Lindl. - Stoechas Don. I. fr.

Heliophila amplexicaulis $\boldsymbol{L}_{\mathrm{i}}$ a.

- crithmifolia Willd. a.

- longifolia DC. a. glabra Rgh. a.

- pilosa Lam. $\alpha$. integrifolia $D C$.

- trifida Thbg. a.

3. incisa DC.a. [a.

Helipterum brachyrhynchum Sind.

Helosciadium leptophyllum DC.a. [a. Heracleum asperum $\boldsymbol{M B}$. p.

- barbatum Ledeb. p.

- dulce Fisch. p.

- flavescens Baumg. a. latifolium $D C$. p.

- granatense Boiss. p.

- Lehmannianum Bge. p.

- ligusticifolium MB. p.

- longifolium Jaeq. p.

- palmatifidum Fisch, Lall. p.

- persicum H. Paris p.

- puhescens $M B$. $\beta$. fructibus marg. scabris p.

- villosum Fisch. p. pum Lall. p.

Wilhelmsii Fisch., Lall. p.

Herniaria diandra $B_{g e}$. a.

- discolor Otto,, Dietr. I. fr.

- odorata Andrz. a. 1 ;

Hesperis matronalis L. $\beta$. a. Ledeb. p.
Hesperis matronalis $\boldsymbol{L}, \boldsymbol{\beta}, \boldsymbol{b}$. Ledeb. fl. alb is p.

Heuchera americana $L$. $\alpha$. robusta Rgl., Rach. p.

- americana $L$. $\beta$.gratilior Rgl., Rach, p.

cylindrica Dougl. p.

glabra Willd. p.

hispida Pursh. p.

- pilosissimit Fisch., Mey. p.

- pubesceus Pursh. p.

- undulata Rigl., Rach. p.

Hibiseus syriacus $L$. I.

Hieracium aestivum Fries. p.

- alpinum L. $\gamma$.Halleri Koch. p.

- andryaloides vill. p.

- auratum Fries. p.

- boreale Fries, p.

- bupleuroides Gmel. p.

- cydoniaefolium Vill. p.

- decipiens Froel. p.

- dentatum Hoppe. p.

- dovrense Fries. p.

- glaucum All. p.

- hispidum Forsk. p.

- longifolium Schleich. p.

- onosmoides Fries, p.

- oreades Fries. p.

- prenanthoides Vill. p.

- rigidum Hartm. p. - $\quad$ 3. coronopi-

folium koch. p.

- Schmidtii Tausch. p.

- speciosum Hornem. p.

- stoloniflorum W., K. p.

- strictum Fries. p.

- tridentatum Fries. p.

Hippocrepis unisiliquosa $\dot{L}$. a.

Horminum pyrenaicum $\boldsymbol{L}$. p.

Hutehinsia alpina $R$. Br.p.

IIyacinthus amethystinus $\boldsymbol{L}$. p.

Hypecoum procumbens $\boldsymbol{L}$. a.

Hypericum Ascyron L. p.

- elegans $S t e p h . p$.

- linearifolium Vahl. p.

- tetrapterum Fries. p.

Jasione montana L. $\beta$. major Koch, b.

Jasminum fruticans $\boldsymbol{L}$. $\mathbf{l}$. fr.

Ilex balearica Desf. I. fr.

Imperatoria Ostruthium $\boldsymbol{L}$. p.

Indigofera australis Willd, I. fr.

Inula Helenium $\boldsymbol{L}$. p. 
Inula hirta $L$. p.

- oculus Christi $\boldsymbol{L}$. p.

- salicina $\boldsymbol{L}$. p.

- suaveolens Jacq. p.

Johrenia dichotoma $D C$. b. fr. Jonopsidium acaule Rchb, a. Iris biglumis Vahl. p.

- Guldenstaedtiana Lep. p.

- ruthenica dit. p.

- setosa Pall. p.

- sibirica $L$. $\beta$. haematophylla Fisch. p.

Isatis canescens $D C$. b.

- oblongata $D C$. b.

- orientalis Willd. b.

Juncus balticus Willd. p.

- compressus Jacq. p.

- Gerardi Loisl. p.

Juniperus excelsa $M B$. I, fr.

Jurinea alata Cass. b. fr.

Kennedya Marryattiana Lindl. I. fr.

- monophylla Vent. I. fr.

- rubicunda Vent. I. fr.

Knowltonia vesicatoria Sims. p. fr. Kochia eriophora Schrad, a. - sedoides Schrad. a.

Krubera leptophylla $\boldsymbol{H o} / \mathrm{m}$. a.

Krynitzkia leiocarpa Fisch., Mey. a.

Lagurus ovatus $\boldsymbol{L}$. a.

Lamarckia aurea Mönch, a.

Lantana alba Mill. I. c.

- hispida Humb, Bonpl. 1. c.

Lapsana intermedia $\boldsymbol{M B}$. b.

Larix dahurica Turcz. I.

- microcarpa Pinet. Wob. 1.

- sibirica Ledeb. pendula I.

Laserpitium gallicum $\boldsymbol{L}$. p.

- hispidum MB. p.

- latifolium L. a.glabrum Koch.

- Siler $\boldsymbol{L}$. p.

Lasiagrostis splendens $K t h$. p.

Lathyrus pisiformis $\boldsymbol{L}$. p.

Lavatera plebeja Sims. b. fr.

- thuringiaca $L, p$.

Leontice altaica Pall. p.

Leonurus glaucescens Bge. p.

Lepidium bonariense $\boldsymbol{L}$. a.

- capense Thbg. a.

- latifolium $\boldsymbol{L}_{\boldsymbol{0}} \boldsymbol{\alpha}$. glabrum $\boldsymbol{L} \boldsymbol{\varepsilon}$ deb. p.

- Menziesii DC. a.
Lepidium micranthum Ledeb. a.

- perfoliatum $L$. a.

- spinosum L. a.

- virginicum $L$. a.

Lepigonum australe $F$. Müll, a.

- canadense Fisch., Mey. a.

- diandrum $\mathrm{Rgl}$. $\beta$. viscidum Rgl. a.

- glandulosum Fisch., Mey. a.

- laxillorum Bartlg. a.

- macrothecum Fisch., Mey. a.

- medium Wahlbg. a.

- mollugineum Lagasc. a.

- rubrum Fries. a.

- segetale Fisch., Mey. a.

Leptochloa arabica $k t h$. a.

Leptopoda brachypoda Torr., Gray. b. fr.

Leptosiphon luteus Benth. a. Lepyrodiclis holosteoides Fenzl. a.

Leucas martinicensis $R$. $B r$. a.

Leucocarpus alatus Don. p. fr.

Liatris spicata Willd. p.

Libanotis buchtormensis $D C$. p.

- condensata Fisch., Mey. p.

- seseloides Turcz. p.

- sibirica C. A. Mey. p.

Ligularia macrophylla $D C$. p.

- sibirica Cass. p.

- speciosa Fisch., Mey. p.

Ligusticum alatum Spr. p.

- $\operatorname{scoticum~L.~p.~}$

- Seguieri Koch. p.

Lilium giganteum Wall. p. fr.

- tenuifolium Fisch. p.

Limnanthes alba Hartw, a.

- rosea Hartw. a.

Limnocharis Plumieri Rich. p. c.

Linaria origanifolia $D C$. b.

Linum angustifolium $\boldsymbol{H}$ uds. a.

Loasa tricolor Ker. a. c.

Loefilingia hispanica $L$. a.

Lonicera coerulea $L$. I.

- tatariea L. 1.

Lotus diffusus Soland, a.

- filicaulis Dur. a.

- gracilis $\boldsymbol{W}, \boldsymbol{K}$. a.

- hispidus Desf. a.

- ornithopodioides $\boldsymbol{L}$. a.

Lupinus nootkatensis Don. p.

Luzula pediformis $D C$. $\mathrm{p}$.

- spicata Desv. p. 
Lycopersicum cerasiforme Dun. a. c. Melilotus italica Lam. a.

Lysimachia punetata Jacq. $\mathrm{p}$.

Malcolmia africana $h$. $B r$. a.

- bicolor Boiss. a.

- Chia DC. a.

- laxa DC. a.

- $\quad$ - $\beta$. stenopetala Rgl. a.

- $\quad$ - . hispidula Rgl. a.

- maritima $\boldsymbol{R}$. $\boldsymbol{B r}$. albillorá a.

- parviflora DC. a.

Malva decumbens Willd. b. fr.

- flexuosa IIorn. a.

- limensis $L$. a.

- mauritiana L. a.

- microcarpa Desf. a.

- nicaeensis All. a.

- neilgherrensis Wight. a.

- pulchella Bernh. a.

- rotundifolia $L$. a.

- verticillata $L$. a.

Malvastrum carpinifolium A. Gr, a. c. - coromandeliaunm Garke. a, c.

Mammillaria amoena Hopf. 1, c.

- chrysacantha Otto. I. c.

- columnaris Mart. l. c.

- crassispina Pfr. l. c.

- dolichocentra Lem. I. c.

- fulvispina Iraw. l. c.

- $\quad$ - 3. rubescens Salm. I. c.

- polythele Mart. I. c.

- $\quad$ - $\beta$.quadrispina Salm. I.e.

- - - .latimamma Salm.1.c.

- pulchra Haw. I. c.

- pusilla DC. 1. c.

- raphidacantha Lem. I. c.

- rhodantha $L k$., Otto. I. c.

- tetracentra H. Berol. l. c.

- Wildiana Otto. I. c.

Manulea Cheiranthus $\boldsymbol{L}$. a.

Marsypianthes hyptoides Mart; a. c.

Maruta microcephala Schrenk. a.

Matricaria nigellaefolia $D C$. p. fr.

- confusa Fisch., Mey. a.

Matthiola parviflora $R$. $B r$, a.

- tricuspidata $\mathrm{k}$. $\mathrm{Br}$. a.

Medicago glutinosa $M B$. p.

Melampodium divarieatum DC. a.

. - longifolium Brouss, a.

- perfoliatum $\boldsymbol{I}$., $\boldsymbol{B} ., \boldsymbol{K}$.: a.

Melandryum brachypetalum Fenzl.p.

Melanthera deltoidea Kich. a.

Melia Azedarach L, I. fr.
- macrorliza Pers. a.

- pallida Bess. a.

1- parviflora Desf. a.

Meniocus linifolius $D C$. a.

Mercurialis ambigua $L$. fil. a. c.

Mesembrianthemum cordifolium $\boldsymbol{L}$.

fil. a. c.

j - oligandrum Kze. a. c.

Meum athamanticum $L$. p.

Micropus californicus Fisch., Mey, a.

- supinus $L$. a.

Microtea debilis Sweet. a. c.

Mimulus moschatus I.indl, a. . I

Mitella diphylla L. p.

- pentandra Honk. p.

Moehringia pentandra Gay. a.

Moenchia quaternella Ehrh, a.

Mollugo verticillata $L$, a.

Alolopospermum cicutarium $\mathrm{FC}$. $\mathrm{p}$.

Jonocosmia corrigioloides Fenzl. a.

Monolepis chenopodioides Moq.

ß. trifida Moq. a.

Montia fontana L. a.

Moschosma polystachyum Benth. a.

Mulgedium alpinum Cass. p. [c.

$\therefore$ - Plumieri DC. p.

- prenanthoides DC. p.

Myagrum perfoliatum $L$. a.

Myogalum Boucheanum Kth. p.

, - nutans $L . k$, p.

Nasturtium clandestinum $S p r$. a.

- micropetalum Fisch., Mey. a.

- polymorphum Nutt. a.

- pyrenaicum R. Br. a.

Nemesia chamaedryfolia Vent. a.

- floribunda Lehm, a.

- versicolor E. Mey, a, '.

Nepeta biloba Hochst. p.

- nudaL. $\alpha$.grandilloraBenth.p.

- B.parvillora Benth. p.

Neslia paniculata Fisch., Mey. a.

Nicotiana fruticosa $L$. b. fr.

Nonnea alba DC. a.

- flavescens C. A. Mey., a.

- Iutea Rchb, a.

- versicolor Fisch., Mey, a,

Notobasis syriaca Cass. a.

Notoceras canariense $\boldsymbol{R}$. $\mathrm{Br}$. a.

Nymphaea alba $\boldsymbol{L}$. p. (Altai).

- caerulea Savign. p. c.

- dentata Schum., Thomn, p. c. 
Ochthodium aegyptiacum DC. a. Ocimum micranthum Willd, a. c. - pilosum Willd, a. c.

Octadenia lybica $R$, Br. a.

Ononis reclinata $L$. a.

- serrata Forsk. a.

- sicula Guss. a.

Orlaya platycarpa Koch. a.

Ormenis mixta $D C$. a.

Orobus aureus Stev. p.

- lathyroides $L$. p.

- luteus L. p.

- niger $L . p$.

- vernus $L . \beta$. flaccidus Koch.p.

Oryza sativa $L$. aristata a. c.

Oralis Dillenii Jacq. a. mutica a. c.

- villosa $M B$, a.

Oxytropis deflexa $D C$. p. fr.

Paederota Ageria $\boldsymbol{L}$. p.

Paeonia anomela $L$. carpell. pubesc. p.

- arietina Anders. p.

- decora Anders. p.

- humilis Rets. p.

- lanceolata Salm. p.

- mollis Anders. p.

- paradoxa Anders. p.

- peregrina Mill. fulgens. p.

- - - humilis p.

- pubens Sims. p.

- triternata Pall. p.

- villosa Sweet. A. albis p.

Paliurus aculeatus Lam. I. fr.

Panicum eriogonum Schrad. a.

Papaver alpinum $L$. a. nudicaule Fisch., Mey. p.

- amoenum Lindl. a.

- commutatum Fisch., Mey. a.

- horridum DC. a.

- hybridum $\boldsymbol{L}$. a.

- orientale $\boldsymbol{L}$. p.

- setigerumDC. B.muticumRgl.

Parietaria alsinefolia Del. a.

- micrantha Ledeb. a.

Paronychia echinata Lam. a.

Parthenium Hysterophorus $\boldsymbol{L}$. a. c.

Patrinia sibirica Juss. p.

Pedicularis elata Willd. p.

Pelargonium australe Jacq. a.

Pentameris airoides $\boldsymbol{N}$. a. $\boldsymbol{E}$. a.

Peplis biflora Salzm. a.

Perilla ocimoides $L$. a. c.

Peucedanum austriacum Koch. p.

- officinale L. p.

- Oreoselinum Mönch. p.

- rablense fioch. p.

Phaca frigida $L$. p.

Phacocapnos Cracea Bernh, a. c.

Phillyraea latifolia L. I. fr.

Phleum annum $M B$. a.

- asperum Vill. a.

- tenue Schrad. a.

Phlomis alpina Pall. p.

Phyllanthus Cantoniensis Hern. a. c.

Physocaulus nodosus Tauseh. a.

Physolobium carinatum Benth. 1. fr.

Phyteuma Scheuchzeri All. p.

- Michelii Bertol. $\alpha$. betonicifolium koch. p.

Picridium hispanicum Poir. a.

- intermedium C.H.Schultz. a.

- vulgare Desf. a.

Pimelea drupacea Labill. I. fr.

Pinus alba Ait. 1.

- Cembra L. I.

- Pinaster Ait. 1.

ß. pumila Pall. 1.

- Pinea L. 1. fr.

Plantago Hookeriana Fisch., Mey. a. Platylobium triangalare $\boldsymbol{R}, \boldsymbol{B r}$. 1 . fr.

Platystemon californicum Benth. a.

Poa paradoxa Kar., Kir. a.

- persica Trin. a.

Podolepis chrysantha Endl. a.

- gracilis Grah. a.

Podotheca angustifolia Cass. a.

- gnaphaloides Grah. a.

Polemonium hamile Willd. p.

Polycarpaea latifolia Poir. a. c.

Polycarpon tetraphyllum L. fil. $\beta$. di-

phyllum DC. a.

Polyenemum majus $\mathrm{A}$. Br. a.

Polygala cordifolia Thbg. I. fr.

Polypogon mexicanus Schlechtd. a.

[a.

Potentilla collina Wib. p.

- De Tommasii Ten. p.

- gariepensis Eckl. a.

- kurdica Boiss., Hohenack. p.

- supina $\boldsymbol{L}$. a.

Poterium lateriflorum Cass. p.

Prasium majus $L$. 1 . fr.

Primula officinalis Jacq. $\beta$. inflata Ledeb. p. 
Psidium litorale Raddi. I. c.

Psychine stylosa Desf. $\beta$. longibrapteata $\mathrm{Kgl}$. a.

Ptarmica sibirica Ledeb. p.

Pteroneurum graecum'DC, a:

Pteropogon chilense Fisch., Mey. a. majus Fisch., Mey. a.

Pterostegia drymariotdes Fisch., Mey. a. c.

Pultenaea tenuifolia $R, B r .1$. fr.

- relusa $\mathrm{Sm}$. l. fr.

- thymifulia Sieb. I. fr.

Puschkinia scilloides Adams.'p.

Pyrethrum caucasicum Willd. p.

Pyrus baccata L. I.

fructib. majorib. I.

- cerasifera Tausch. I.

- $\quad \gamma$. Calvillea Tausch.l.

Ranunculus arvensis $L$. $\beta$. tuberculatus Koch, a.

- lomatocarpus Fisch, Mey. a.

- muricatus L. a.

- nodiflorus $L$. a.

- ophioglossifolius Vill. a.

- parviflorus $L$, a.

- Philonotis Fhrh, $\beta$. intermedius DC. a.

- Schraderianus Fisch., Mey, a.

- trachycarpus Fisch., Mey. a.

- trilohus Desf. a.

Rapistrum rugosum $A l l$, silicúlis glabris Koch. a.

Rhamnus Alateruus L. I,

Rhaponticum puichrum Fisch., Dey.

- s ariosum Lam. p.

Rheum hybridum Ait. p.

Rhynchopsidium sessiliflorum $D C$.a.

Ribes aciculare $S \mathrm{~m}$. I,

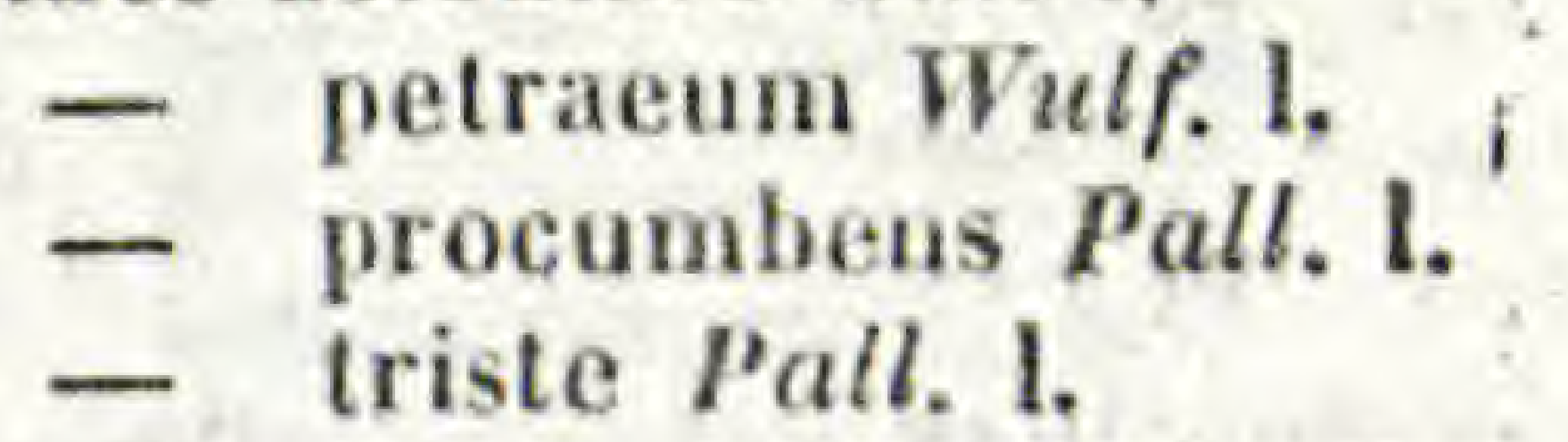

Rudbeckia speciosa Wender. p.

Rulingia corylifolia Grah. I. fr.

Rumex Gmelini Turcz. p.

- stenophyllas Ledeb. p.

Sagina apetalia $\boldsymbol{L}$. a.

- maritima Don. (Rchb.) a.

- saxatilis Wimm. a. *

- stricta Fries. a.

- subulata Wimm. a.

Salvia aurita Thbg. I. fr.

- spinosa $\boldsymbol{L}$. a.
Sanguisorba media $L$, p.

- sitchensis C. A. Mey. p.

- tenuifolia Fisch. p.

$-\quad$ a. leucantha $C . A$.
Mey. p.
$-\quad$ B. rhodantha $C . A$.
Mey. p.

Saponaria calabrica Guss, a.

- cerastoides Fisqia. a.

- orientalis $L$. a,

- porrigens $L$. a.

Saussurea amurensis Turcz, p.

Saxifraga Aizoon L, p.

- nivalis $L$. p.

Scabiosa caucasica $M B$. p.

Schistanthe peduncularis Kze, a.

Schizopetalum Walkeri Sims. a.

Scilla cernua Red, a. pauciovulata - verna Huds. p.

11. roseis $p$.

[ligl.p.

Scleropus amarantoides $\boldsymbol{S}$ chrad, a. c. Scrophularia divaricata Ledeb. p.

- Iuridillora Fisch., Mey. p. fr.

- Scopolii IIoppe. p.

- smyrnaea Boiss. p. ar.

- vernalis $\boldsymbol{L}$. p.

Scutellaria alpina $L$. p.

Sedum Aizoon L. p.

- annuum $L$. a.

- atropurpureum Turcz. p.

- Beckeri C. A. Mey. p.

- Cepaea L. a. galioides DC. a.

- Ewersii Ledeb. p.

- hybridum L. p.

- involucratum $\boldsymbol{M B}, \mathrm{p}$,

- kamtschaticum Fisch. p.

- leptorhizum C. A. Mey. p.

- oppositifolium Sims. p.

- pallidum MB.,a.

- purpurascens Koch. p.

- Rhodiola DC. p. - $\delta$. viride Ryl. in 11. ajan. russ. (S. elongatum

Ledeb. p.).

- spurium $M I B$. p.

- stellatum $I$. a.

Senebiera didyma Pers. a.

- linoides DC. a,

Senecio macrophyllus $M B$. p.

- octoglossus DC. p.

- palmatus Pall. p.

Serralula Gmelini Ledeb. p. 
Serratula radiata $\boldsymbol{M B}$. p.

Seseli montanum $D C$. p.

Sibbaldia cuneifolia Edgew, p.

Sideritis romana $\boldsymbol{L}$. a.

Silene alpestris Jacq. p,

- ambigua Camb, a.

- annulata Thore. a.

- antirrhina $\boldsymbol{L}$. a.

- apetala Willd. a.

- brachypetala Rob. Cast. a.

- compacta Fisch. b.

- conoidea $L$. a.

- disticha Willd. a.

- diurniflora Kze, a.

- fimbriata Sims. p.

- flavescens $W ., K$. p.

- fuscata $L k$. a.

- gallica $L$, a.

- gonocalyx Boiss. a.

- graminifolia Otth, p.

- italica Pers. b.

- juvenalis Del. a.

- linifolia Willd. a.

- muscipula $L$. a.

- nicaeensis All. a.

- noctiflora $\boldsymbol{L}$. a.

- nocturna $\boldsymbol{L}$. $\beta$. paucifloraOtth.

- orientalis Mill. b. fr. [a.

- ornata Ait. a.

- repens Patrin. a.

- rabella $L$. $\beta$. brachypetala Mo-

- Saxifraga $L$. p.

- tatarica Pers. p.

- tenuiflora Guss. a.

- tridentata Desf. a.

- trinervia Sebast. Maur. a.

- velutina Pourr. I. fr.

- vespertina Retz. a.

Sinapis abyssinica $A$. Br. a.

- Allionii Jacq. a.

- hispida Schousb. a.

- integrifolia Willd. a.

- juncea $\boldsymbol{L}$, a.

Sisymbrium bursifolium $\boldsymbol{L}$. a.

- Columnae Jacq. a.

- Cumingianum Fisch, Mey. a.

- hirsutum Lag. a.

- Irio $L$. a.

- nitidum Zea. a.

- pumilum Steph, a.

- subhastatum Willd. a.

Sonchus hypochaeroides Schltd. a.
Sonchus laevigatus Willd. 1. fr.

Sophora japonica $L$ I. fr.

Spartium junceum L. 1. fr.

Sphaerolobium fornicatum Benth. I.

- medium $R$. Br. 1. fr.

[fr.

- vimineum Sm. l. fr.

Sphaerostigma minutiflorum Fisch., Mey. a.

- strigulosum Fisch., Mey. a.

Spiraea laevigata $L$. I.

Statice speciosa $L$. p.

Stellaria viscida $M B$. a.

Sterculia platanifolia $L$. fil. l. fr.

Stipa tortilis Desf.' a.

Stylidium adnatum $R$. Br. p. fr.

Suaeda gracilis Moq. a.

Succovia balearica Medik. a.

Tauscheria lasiocarpa Fisch. a.

Teloxys aristata Moq. a.

Tetrapoma barbareaefolium Turcz.b.

Thalictrum angustifolium Jacq. p.

- $\quad$ - $\gamma$. laser-

pitiifolium Koch. p.

- appendiculatum C. A. Mey. p.

- elatum Jacq. p.

- foetidum L. $\beta$. glabrum Koch.

- glaucum Desf. p.

- Jacquinianum Koch. p.

- kemense Fries. p.

- medium Jacq. p.

- minus L. p.

- $\quad$ - . koch. p.

- mucronatum Ledeb. p.

- nutans Desf. p.

- rugosum Ail. p.

- simplex L. p.

- sylvaticum Koch. p.

- trigynum Fisch. p.

Thermopsis fabacea DC. $\mathrm{p}$.

Thisantha glomerata Eckl., Zeyh.a.c.

- - patens Eckl., Zeyh. a, c.

Thomasia purpurea Gay. l. fr.

Thysanocarpus pulchellus Fisch., Mey, a.

Tillaea muscosa $L$, a.

Tordylium syriacum $L$, a.

Torilis nodosa Gaertn. a.

Tradescantia erecta Jacq. a. c.

- undata Willd. a. c.

Tragopogon barbirostris Bisch. b.

- coloratus C. A. Mey. b.

Tragopogon major Jacq. b. 
Tragopon orientalis $L$. $b$.

- parviflorus Hornem. b.

Trautvetteria palmata Fisch., Mey.p.

Trichogyne verticillata Less, a.

Trichonema ramillorum Sweet.p.fr.

Trifolium cernuum Brot. a.

- Cherleri L. a.

- diffusum Ehrh. a.

- erinaceum $M B$. a.

- filiforme L. a.

- glomeratum $L$. a.

- Gussoni Tineo. a.

- hirlum All. a.

- incarnatum $L$. a.

- intermedium Guss, a.

- latinum Sebast. a,

- leucanthum $M B$. a.

- ligusticum Balb. a.

- maritimum Huds. a.

- Michelianum Savi. a.

- microcephalum Pursh. a.

- minus Sm. a.

- niditicum Griseb. a.

- nigrescens Viv. a.

- pallidum $\boldsymbol{W}, \boldsymbol{K}$. a.

- parviflorum Ehrh. a.

- procumbens $L$, a.

- resupinatum $L$. a.

- rotundifolium Sibsh., Sm. a.

- scabrum L. a.

- Sebastiani Savi. a.

- spunosum L. a.

- squarrosum $L$. a.

- stellatum L. a.

- striatum L. a.

- strictum $L$. a.

- suaveolens Willd. a.

- subterraneum $\boldsymbol{L}$. a.

- succinctum Vis. a.

- tomentosum L. a.

Trigonella anguina Del. a.

- calliceras Fisch, a.

- cancellata Desf. a.,

- Foenum graecum'L. a.

- glomerata II. Paris. a.

- monantha C. A. Mey. a.

Trochocarpa laurina $\boldsymbol{R}$. Br. l. fr.

Trollius altaicus $C$. A. Mey. p.

- caucasicus Stev. D.

Tunica Sàxifraga Scop, p.

Tunica stricta Fisch., Mry. b.
Umbilicus pendulinus DC, p. fr.

Valeriana alliariaefolia $\mathrm{Vahl}$. p.

- exaltata Mik. p.

Valerianella echinata $D C$, a.

- eriocarpa Desu. a.

- hamala DC. a.

- hirsutissima Lk. a.

- microcarpa Loisl. a.

- Morisonii DC. a.

- oxyrhyncha Fisch., Mey. a.

- plagiostephana Fisch., Mey.a.

- pumila DC. a.

- Soyeri Buchinger. a.

- vesicaria Mönch, a.

Veratrum album L. $\beta$. flor, virid,

Veronica biloba $L$. a. [Cham. p.

- Buxbatinii Ten. a.

- ceratocarpa C. A. Mey. b.

- Cymbalaria Bertol. a.

- opaca Fries. a.

- pinnata L. p.

- sibirica L. p.

; - spicata $L$. var. $\left(Y_{i}\right)$ australis Schrad. p.

Vesicaria corymbosa Griseb. a.

- grandillora Ilook. a.

Vicia amoena Fisch. p.

- atropurpurea Desf. a.

- bithynica $\boldsymbol{L}$. a.

- Bobartii Forst. a.

- calcarata Desf. a."

- grandillora Scop, ;a.

- hybrida L. a.

- hyreanica Fisch., Mey. a.

- lutea L. B. pallidiflora DC. a.

- - $\quad$ - glabra Rgl. a.

- Michauxii Spr. a.

- monantha Koch. a.

- pannonica Jacq. a.

- peregrina $L$, a.

- polyphylla Desf.. a.

- Pseudocracca Bertol, a.

- segetalis Thuill. a.

Vincetoxicum medium Decsne, p.

Vitex Agnus castus $\boldsymbol{L}$. I. fr.

Wahlberzella affinis C. A. Mey. p.

- apetala Fries. p.

Xanthogalum purpurascens Lall, p.

Youngia diversifolia Ledeb. p.

Ziziphora clinopodioides Lam. p. 


\section{S E M IN A}

\section{IN \\ REGIONE WOLGENSI PROPE SAREPTA LECTA.}

Abutilon Avicennae Gaertn. Acroptilon Pieris C. A. Mey. Alhagi camelorum Fisch. Allium tulipaefolium Ledeb. Althaea taurinensis $D C$. Asperula humifusa Dess. Astragalus albicaulis $D C$.

- asper Jacq.

- longillorus Pall.

- macropus Bge.

- physodes $\boldsymbol{L}$.

- virgatus Pall.

- vulpinus Hilld.

A triplex acuminata $W ., K$.

- tatarica $\boldsymbol{L}$.

Bunium luteum $\boldsymbol{H}$ offm,

Calophaca wolgarica Fisch.

Carduus uncinatus $\mathbf{M B}$.

Centaurea adpressa Ledeb.

- arenaria $\boldsymbol{M B}$. glastifolia $\boldsymbol{L}$.

- inuloides Fisch.

- ruthenica Lam.

Cephalaria centauroides Coult. Chaerophyllum Prescottii $D C$.

Cirsium esculentum C, A. Mey.

- serrulatum $M B$.

- setigerum Ledeb.

Crataegus ambigua,

Delphinium puniceum Pall.

Dianthus capitatus $\boldsymbol{D C}$.

Eehinops Bitro $\boldsymbol{L}$. var teuuifolius.

Elymus sabulosus $\boldsymbol{M B}$.

Ephedra monostachya $\boldsymbol{I}$.

Eragrostis suaveolens $\boldsymbol{M B}$.

Eriosynaphe longifolia $D C$.

Ferula caspica $M B$.

Fritillaria ruthenica Wikstr.
Galatella dracunculoides $\boldsymbol{N}$. a. E.

$\beta$, punctata.

Galium tataricum Trev,

Glycyrrhiza glandulifera $W, K$.

Gypsophila paniculata $\boldsymbol{L}$.

Halimocnemis crassifolia $\mathrm{C}, \mathrm{A}, \mathrm{Mey}$.

- Volvox C. A. Mey.

Heracleum sibiricum $\boldsymbol{L}$.

Iris aequiloba Ledeb.

- tenuifolia Pall.

Juriuaea Eversmanni Bge.

- polyclonos $D C$.

Libanotis sibirica C. A. Mey.

Linaria genistaefolia AIIll.

- odora Chav.

Linum austriacum $L$, squamulosum.

Linosyris villosa $D C$.

Lotus tenuifolius Presl.

Melilotus ruthenica $M B$.

Microphysa rubioides $C$. A. Mey.

Nitraria Schoberi $\boldsymbol{L}$.

Ononis intermedia $C, A, M e y$.

Onosma tinctorum $M B$.

Palimbia salsa Bess.

Pastinaca graveolens $\boldsymbol{M B}$.

Peucedanum latifolium $D C$.

- sibiricum Claus,

Phlomis pungens Willd.

Plantago Cornuti Gowan.

Potentilla astracanica Jacq.

Rindera tetraspis Pall.

Rumex stenophyllus Ledeb.

- ueranicus Fisch.

Rumia laevigata.

Salsola laricina Pall.

- mutica C. A. Mey.

- Soda $L$,

Saussurea crassifolia $D C$. 
Scabiosa ucranica $\boldsymbol{L}$.

Schoberia acuminata C. A. Mey.

Scorzonera caricifolia Pall.

Serratula Gmelini Ledeb.

- xeranthemoides $M B$.

Silaus Besseri $D C$.

Silene wolgensis $S p$.

Sinapis juncea $I$.

Statice caspia Willd.

- incana $L$.

- latifolia Sm.

- sareptana A. Becker.

- suffructicosa $L$.

- tatarica $L$.
Statice tomentella Boiss.

Syrenia Lamarckiana Andr.

Thalictrum collimum Wallr.

- commutatum C. A. Mey.

- mucronatum Ledeb.

Tournefortia Arguzia R.S.

Triticum desertorum Fisch.

- rigidum Schrad.

Tulipa biflora $\boldsymbol{L}$.

- Gesneriana $L$.

- tricolor Ledeb.

Valeriana sambucifolia Mikan.

Verbascum orientale M.R.

Vicia picta Fisch., Meyl. 


\section{ANNOTATIONES BOTANICAE.}

\section{Smilax grandifolla mgl.}

Scandens, glabra; ramis quadrangulis sparse aculeatis; aculeis va. lidis incurvatis; foliis cordato-oblongis vel ovali-oblongis, acuminatis, apice complicato recurvatis, 5-nerviis, nervis subtus prominentibus aculeolatis; umbellis axillaribus vel $2-3$ in ramulos bracteatos terminales dispositis; pedunculo petiolum aequante vel superante.

Rami alte scandentes. Folia inferiora basi cordata, usque 7 poll. longa et $3^{1 / 4}$ poll. Jata; superiora basi rotundata, circiter $41 / 2$ poll. Ionga et 2 poll. lata; omnia 5-nervia, nervis subtus prominentibus utroque extimo tenuiore. Petioli subcontorti, basi vaginati, infra medium (in apice raginulae) bicirrhati, cirrhis longissimis vel subnullis. Umbellae multiflorae. Pedunculi teretes, pollicares. Receptaculum globosum, bracteolatum. Pedicelli $1 / 2$ pollicares. Flores masculi campanulati, flavi. Sepala oblongolanceolata, obtusiuscula, usque 3 lineas longa. Stamina brevia, complanata, antheris linearibus filamenta aequantibus terminata.

Smilaci officinali affinis. Cl. Riedel semina misit e Brasilia. (Rgl.).

\section{Astroemerla psittacina Lehm. Var. longlpodunculata IR gl.}

Pedunculis 4-6, 3-6 pollices longis, interdum bifloris. (Rgl.).

\section{Agave maculata $\mathrm{mgl}$.}

Acaulis, glabra; foliis basilaribus lineari-lanceolatis, recurvo-patentibus, coriaceis, supra canaliculatis, subtus convexis ecostatis, acutissimis, absque aculeo terminali, apice convoluto-teretibus, tenuissime cartilagineo-albo-marginatis et margine dentibus minutissimis cartilagineis instructis, laete viridibus et utrinque fusco-maculatis; scapo 3 -pedali, inferne foliis superne squamis distantibus munito, apice racemum simplicem pauciflorum gerente; floribus pedunculatis, erectis, ex viridi purpurascentibus, basi squamis lanceolato subulatis suffultis; corolla infundibuliformis, supera, 6-fida, apicem versus purpurascente, 1 poll. longa, germen cylindrieum interdum squamis munitum aequante, laciniis lanceolatis; staminibus 6 corollae tabo adnatis, longe exsertis, purpurascentibus; antheris linearibus dorso affixis; stylo purpurascente staminibus aequilongo.

Affinis A. rubescenti Salm Dyk, revolutae Kl., Saponariae Lindl. etc. Semina misit cl. Karwinski e Mexico. (Rgl.).

\section{Agave Saponarla l.tndi.}

Cl. Klotzsch hane speciem conjunxit cum A. bracbystachy Cav. (Allgem. Gartenz, 1840, 274). Planta nostra dignoscitur ab A. brachy- 
stachy Cav, (A, spicata Red, Lib. lab, 485 nec Cav.) foliis laxis, linearilanceolatis, recurvis, pedem longis, pollicem latis, margine sub lente cartilagineo-serrulatis apice margine convolutis acutisve; floribus $10-12$ spicam laxam formantibus (nec densis nee subimbricatis), (1)gl.),

\section{Aeohmeg fulgene A. Brongn. $\beta$. Alomgrata Mgt,}

(A. minịala Hart. Van, Houtte).

Foliis minutissime spinuloso-denticulatis; panicula abbreviata, glomerata, ad apicen ramosa. (Rgl.).

\section{Buberala horrda regl. (B, latifolia Hort. Petrop,),}

Glabra; foliis linguaeformibus, latis, concavis, ex apice obtuso apiculatis, margine grose dentato spinosis; scapo folịa superante, viridi, foliis laxis, scariosis, demum brutheis vestito; racem erecto, nudo; bractea infima scariosa, supeqioribys brevissimis rolundato- $q$ btusis squamaeformibus viridihus; lloribus sessilibus; calicis laciniis truncato-bbtusis, superne pilosulis; petalis basi utrinque squama hyalina apice eroso-laciniata munitis; germine elongato. glaberrimo; stigmafibus tribus recurvato patentibus, linearibus.

Folia obscure viridia, 8 pollices longa, 2 pollices fata, spinis, fuscescentibus. Calyx viridis. Petala viridi-lutoa apice coerulescentia, $11 / 2$ poll. longa, calyce subtriplo longiora. - An genus proprium? (Rgl.),

\section{Caraguata serrata $\mathrm{ar}$. et $\mathbf{s}$.}

Scapo folioso vix pedali; foliis a pasi latiore vaginante elongatolineari-lanceolatis, $1 \frac{1}{2}$ pedes longis, $1-1 \frac{1}{4}$ poll. latis, acutissiphis, laete viridibus, glapris, breviter aculeatb-dentatis, dentibus subincuryis, foliis superioribus minoribus; bracteis, lanceolatis, coccineis; Rorihus in corymbos axillares plerumque triflores practea multo braviores congestis; calyce obtuso, trigono, trifido, laciniis acutiusculis; corolla monepetala, calycem triplo superante, basi albida superne pulchre coerulea triloba, lohis orectis cucullato-obtusis; staminihus corollage tubo adnatis, antheris linearibus liberis dorso affixis basi breviter sagitlatis corolla brevioritus; stigmate trilobo, lohis spiraliter lortis. (Rgl.).

\section{Pleurothallin lanmbergil agl. (Aggregatae, laeves, cordatae),}

Caule secundario $1 / 2$ pedali, téuni, teretiusculo, internodio superiore Jongissimo; folio pergameno, ovato-oblongo vel oblongo, hasi cordato, apice acuminato integro vel tridentato; bracteis vaginantibus truncatis hyalinis; floribus pillide fuscis $1-2$; sepalis 2 trinepviis $(1 / 2$ pollicem longis), superiore ovato lanceolato acuto, inferiore subaequali paullo latiore; petalis anguste linearibus, quam sepala dimidio breviọibus; labello pelalis aequali, brevifer unguiculato, e basi subhastata oyato-linguiformi, duplo longiore quam láto, crasso, densissime muriculato, atropurporeo; gymnostemio abibreviato, truacato.

Folia usque $3^{\frac{1}{2}}$, pollices longa, $1^{1 / 2}$ pollicem lata. Proxime accedit ad PI, Cardium Rehb., quae differt racemo 6-10 floro et labelli forma, Cl. Lansberg e Columbia misit. (Kbl.), 


\section{gleurothaltis loranthophylla akehb. Al. Var. pelluelda iggl.}

Floribus hyalino-luteolis pellucidisque, purpureo-punctalis. - Reliqua ut plantae genuinae. An Pl. subpellucida KI.? Rhynchopera punctata Karst. Ausw. Lab. VII, cum Pl. loranthophylla Rehbch. fil. congruit. (Rgl.).

\section{Oetomerla mentteres agl.}

Proxime affinis Octomeriae Chamaeleptoti Rchbch. fil., differt tamen foliis semiteretibus, supra canaliculatis, apice pungenti-subulatis; floribus 1-8 aggre ratis; sepalis obtusis; labelli ungue sacciforme, apice hastatutrilobo, lobis lateralibus cum intermedio confluentibus; intermedio oblongo, apice obtuso, disco carinis duabus elevatis munito.

Caules secundarii filiformes, vaginis scariosis munili, usque 3 poll. longi. Folium unicum in caulis apice, carnosum, semiteres, supra canaliculatum, paullo recurrum, apice subulato-attenuatum, pungens, glabrum, usque 5 poll. longum el $1 \frac{1}{2}$ lin. latum. Flores $1-8$ in folii axilla aggregati, pallide flavi, hyalini, 4-5 lineas in diametro. Sepala oblonga, obtusa. Petala similia, paullo minora, ex apice obtuso acutiuscula. Labellum sepalis duplo minus, basi sacculo unguiformi, superne in laminam trilobam e basi hastata oblongam obtusam desinens; lobis lateralibus obtusis, auriculiformibus in lobum medium desinentibus et basin hastatam laminae formantibus; lobo medio lateribus recurvis, apice rotundato, basin versus utrinque carina elevata munito. Columella semiteres, gracitis, basi producta et cum labello articulata. Pollinia 8 , quaternatim cohaerentia.

Habitat in Brasilia, (Rgl.).

\section{mestrepia vittata Lindl. Var binora hol.}

Pedunculis geminis folio $4-5$-plo brevioribus; labello linguiformi, acuto; reliquis ut in planta genuina.

Caules teretiusculi, cespitosi, crassitie pennae corvinae, $4-b$ pollices longi, superne folium unicum gerentes. Folium oblongum, obtusum, crassum, 4 pollices longum, $1 \frac{1}{2}$ pollices latum. Pedunculi ad basin folii gemini, folio 4 - Splo breviores, bractea membrauacea fulti. Flos ringeus. Sepala 2, opposita, albida; superius lanceolato-elongatum, acuminatum, lineis 3 rubris notatum, $7 / 8$ pollicem longum, $1 / 4$ pollicem latum; inferius subduplo latius. Petala e basi latiore ciliata, filiformi-elongata, sepalum superius subaequantia, linea purpurea notata. Labellum linguiforme, acutum, ciliatum, laciniis lateralibus auriculiformibus obtusiusculis, fuscopurpureum, lineis 4 pallidis notatum, petala aequans. - An species propria?

Cl. Lansberg plantas vivas e Columbia misit. (Rgl.).

CIparls elata c.Indl. $\beta$. purpurascens $\mathbf{a g l}$

Folis et scapis purpuraseentibus, bracteis erectis. ( $\mathbf{H}_{\mathrm{b}} \mathrm{l}$.).

Epldendrum alatum Batem. $\beta$. parviflorum Egt.

Rhachis atque germina et plantae nostrae et in figuris (nec in descr.ptionibus) tab. 3898 Bot. Mag. tabulaeque 18 Bat, Orch. Mex. verruculosa. Hanc ob causam pertinel E. alatum ad sectionem Lindleyanam 2, Eatsium $\mathbf{D}$, Hymenochylia b, lobo labelli intermedio obtuso, rhachi 
aspera, neque ad sectionem 'thachr laevi, cui cl. Lindley hanc plantam atiumeravit, Variat:

a. longipetalum; floribus usque $2^{1 / 2}$ poll. in siametro tendentibus; sepalis petalisque lineari-spatbulatis, fusco purpureis; labelli lobis lateralibus llavo-viridibus, lobo intermedio albo luteo-marginato el purpureo-striato.

E. alatum Batem. Orch. Mex. tab. 18.

E. longipelalum Lindl. Paxt. Flow. Gard. I., t. 30.

3. grandiflorum; floribus viridi-flavescentibus; sepalis petalisque apice fusco-purpureis; reliquis ut in praecedente.

E. calocheilum llook. Bot. Mag. 3898 .

$\gamma$. parviflorun; floribus $1 \frac{1}{3} \cdot$ poll. in diametro tendentibus, viridiIuteis et fusco-variegatis; petalis sepalisque spathulato-oblongis, labelli lobis lateralibus viridi-flavescentibus purpureo-striatis, lobo intermedio luteo, disco albido obsolete striato. (V. in hort. Petrop. plantam viram).

$\delta$. viridiflorum; lloribus $1 \frac{2}{3}$ poll. in diametro, virescentibus: petalis sepalisque oblongo cuneatis acutiusculis; labello ex albido virescente, disco albido, lohis lateralibus et lobo intermedio striatis.

E. macrocheilum Gal. e Mexico. (Rgl.).

MaxtIaria leptosepala líook.' $\beta$. Nublntegerrinu ugi.

Differt labello subintegerrimo nec dentato-fimbriato. CI. Lansberg mis. e Columbia, (Rgl.).

Epidendrum odoratlsalmum ratndi. Var. crispum Eat.

Differt panicula ramosa, ramis racemosis; sepalis petalisque viridibrunneis; labello citrino purpureo-striato-venoso, lobo intermedio crispo. (R.l.).

\section{Epldeadrum stamfordlanum Batew. $\beta$, parviftorum Bgl.}

Floribus minoribus; labelli lobo intermedio denticulato nec ciliato. (Rgl.).

\section{Maxillarla'Galeottiana ikgl.}

Pseudobulbis oblongis, apicem versus attenuatis, profunde sulcatis, vix compressis, hasi squamis membranaceis fuscis involutis, apice folia 1-2 lineari-lanceolata mucronato-acuta gerentibus; scapis in squamarum axillis, quam pseudobulbi plas duplo longioribus, unilloris, rubris, vaginis 4 membranaceis remotis vestitis; sepalis allenuato-oblongis, acutis, erectopatentibus; petalis paulio brevioribus et duplo angustioribus; labello oblongo, trilobo, lobis obtusis, intermedic ablinguiformi revoluto nudo, disco tubercuto oblongo.

Pseudobulbi usque $1 \frac{1}{3}$ poll. longi et $1 / 2$ poll. lati. Folia usque 1 pedem longa et $1 / 3$ poll. lata. Flores pollicem in diametro, ochroleuci, purpureo-variegati. Labellum petalorum longitudine, basi pulchre rubrostriatum, superne sanguineo-marginatum et punctalum. - Planta pulchra, a cl. Galeotti e Brasilia accepta, Affinis M. acutipetalae Hook., sed bulbi et folia tenuiora et flores dupld minores. A M. angustifolia dignoscitur labelli forma. ( $\mathbf{H} g \mathrm{l}$.$) .$

Maxillarla guarelmenis Kehh. A. (in Bonpl. II., p. 16.

Accepimus a Wagenero e Columbia nostram plantam, quae certissime species anteposita est. A diagnosi Reichenbachiana differt: foliis 
6-10 poll. Iongis, ex apice oblique retuso apiculatis; pseudobulbis basllaribus et in apice caullum distiche foliatorum terminalibus; phyllis pe rigonii interioribus quan exteriores $1 / 4$ brevioribus: labello integro, lineari-ligulato, superne recurvo, obtuso, basin versus rubicundo. (Rgl.).

\section{Maxillarla IInguiformis ngl.}

Pseudobulbis oblongis, utrinque attenuatis ancipiti-compressis, ecostatis, basi foliis $3-4$ cinctıs, apice folium unicum gerentibus; foliis elongato lineari-lanceolatis, oblusiusculis; pedunculis axillaribus, aggregatis, vaginatis, folio quadruplo brevioribus, unifloris; lloribus ex albidoDavescent bus, e basi latiore lineari-lanceulatis, acutis, margine revolutis; petalis paullo minoribus et duplo angu,tioribus; lahello oblongo, sepalis iripio breviore, obtuse trilobo, lobis lateralibus parvis incurvis, lobo interuedio linguiformi crasso recurvo obtuso subvelutino-piloso, callo oblougo vix elevato pilosulo\} columna semiterete, labello paullo breviore.

Pseadobulbl circiter 1 poll. longi, 1 poll. lati. Folia usque $1 \frac{1}{2} 2$ ped. longa et poll. lata, laete viridia, nitida, Flores „raveolentes. Sepala $1 \frac{1}{4}$ poll. longa, 2 lineas lata. Labelli pars inferior albida, intus purpureoatriulata, lobus anticus aureus. - Afflnis Maxillariae pictae Hook., diversa tamen pseudobulborum et follorum et labelli forma. (Rgl.).

\section{astiliarlia Nitualens thook.}

3. stenopetala Rigl. Flo ibus luteo-carneis; sepalis lanceolatis acutis, lateralibus faleatis; petalis minoribus; labello purpureo.

$\gamma$. obseura Rgl. Ut var. $\beta_{.}$, floribus vero e lilacino ochraceis.

\section{Maxillarla virlalis hladl.}

Variat: $\alpha$. uniflora platysepala Rgl.; pedunculis axillaribus uniDoris; sepalis petalisque subrotaudis conniventibus.

Masillaria viridis Lindl. Bot. Reg. tab. 1510.

$\beta$. Uniflora stenosepala $\mathrm{Kgl.;}$ pedunculis axillaribus unifloris; sepalis petalisque oblongis obtusis.

Maxillaria placanther.a Hook. Bot. Mag. tab. 3173.

Maxillaria cyanotbeile Hoffm. Cat.

\%. pluriflora Rgl.; pedunculis axillaribus 2-3 floris; sepalis ovatis; petalis obverse lancelato-oblongis.

$\mathrm{V}$ ar, $\gamma$, in horto Petropolitano colitur. (Rgl.).

\section{'Tetragamestus Isbchlloldes igl.}

Scaphyglottis arundinacea Hort. Petrop.

Caulibus flaceldis, subteretibus, articulatis, deinde apice fasciculatoramosis, basi vaginis membranaceis cinctis; folifs distichis, alternis, lineari-lanceolatis, apice obtuso bidentatis; floribus terminalibus, (semper?) solitarils; pedunculo bracteato erecto; ovario elongato pedicelliformi. quam flos duplo longiore; sepalis petalisque lanceolatis, acutis; labello e basi cuneata ovato-oblongo, apice retuso, margine undulato, basi bilamellato, sepala paullo superante; columna semitereti, e basi attgustiore superne utrinque auriculata; pollinibus 2 , bipartibilibus.

Caules usque bipedales. Folia $1 / 4-1 / 2$ pedes longa, $1 / 6-1 / 2$ pollicem tata. Flores pallide flavi et albidi, sepalis $1 / 4$ pollicem longis.

Cl. Lansber 
Sianhopea graveolens l.. $\beta$ : Inoulora agh.

Stanhopea remofa Hort. Berol.

Racemo expanso; bracteis ovatis, ovario duplo brevioribus s sẹpalis Jateralibus late ovatis; labello colunyaque ut St. inodorae.

Flores pallide citrini, sanguineo-maculato-punctati. Labelli hypochilium croceum, maculis duabus brumeis maximis. (Rgl.).

An planta hybrida inter Stanhopeam graveolentem et inodoram?

\section{Glanhopea oculata Hindi. Var. erocen Mgl.}

Haec varietas pulcherrima a planta genuina dignoscitur: sepalis late ovatis petalisque saturate croceis rubro maculatis, petalis basi maculis magnis atrosanguineis, labello columnaque ut genuinae sed robustioribus. (Rigl.).

\section{Gomeza FIachori itgl.}

Rodriguezia macrostachya Hort. Petrop.

\section{1. $j$}

Pseudobulbis ovalo-oblongis, ancipiti-compressis; racemis multifloris, recurvis, folium aequantibus vel eo longioribus basi plerumque ramosis, axillaribus et terminalibus; flor bus pallide viridibus; sepalis petalisque incurvato-patentibus, oblongo-lanceolatis, obtusis, crispulis; sepalis duohus inferioribus majoribug $1 / 2$ pollicem longis, foliolum unicum ovatum sublus subbicostatum apice bilobatum formantibus; sepalo superiore petalisque conformibus, $1 / 3$ poll. longis.

Glabra. Pseutobulbi apicem versus attenuati, usque 3 poli. Iongi et $1^{1 / 3}$ poll. lati. Folia 2 , lineari-lanceolata, acuta, striato-nervosa, usque 12 poll. longa et $1 \frac{1}{4}$ poll. lata. Racemus et in axillis vaginarum oppositarum foliacearun basalium ortus ef terminalis, recurvus, nultịlorus, usque 12 pollicaris، Bracteae lineari-lanceolatae, acutae, pedunculum $1 / 3$ poll. longum involventes, inferiores pedunculo sesquilongiores superiores eum vix superantes. Labellum oblongum integrum a medio recurvum, callis duobus membraniformibus unidentatis parallelis ornatum. Colunna brevis semiteres, antice excavata. Pollinia 2 pyriformi-subglobosa, posti e excavata. Caudicula gracilís basi glandulae oblongae insidens. - Afinis G. Barkeri Hort.y planifoliae KI. et suaveolenti KI. 6. Barkeri differt pseudobulbis oblongis, racemo breviore, sepalis petalisque angustioribus et acutis, sepalis inferioribus ad medium tantum coalitis. - G. suaveolens (Pleurothallis foliosa Bot. Mag. 2766) differt racemo erecto breviore, floribus luteis, petalis sepalisque anguste lanceplatis acutis patentissimis, sepalis 2 inferioribus basi tantum coalitis. - G. planifolia Kl. differt racemo semper simplici et axillari, floribus luteis, petalis sepalisuue angustioribus acutis.,

Patria plantae nostrae iguota, probabiliter Brasilia. (Rgl.).

\section{Gomean planifolla Ki.}

Var. a. laxa $\mathrm{Rgl}$.; floribus virescenti-luteis, in racemum laxum foliis lougiorem dispositis, (But. Mag. 1. 33̈04).

Var. $\beta$. densa Rgl.; floribus deinde pulchre luteis, in racemum densum foliis $1 / 3-1 / 2$ breviorem dispositis. (R $/$ l.). 


\section{- $12-$ \\ Dneldium eltrinam CIndi.}

a. verum; (Lindt. Bot. Reg. tab. 1758) sepalis petalisque obscure brunneo-maculatis; labelli lobis lateralibus auriculiformibus et postice productis.

3. rotundatum $\mathrm{Rgl.;}$ sepalis petalisque brunneo-maculatis; labelli lobis lateralibus rotuudatis, postice non productis.

Accepimus hauc varietatem a cl. lansberg e Columbia. (Rgl.).

Dneldium pulvinatum Lindl. $\beta$. graudiflorum gagl.

Differt floribus majoribus ( $1 \frac{1}{3}$ poll. in diametro tendentibus); labelli lobis lateralibus laciniato-dentatis. (Rgl.).

Dncidium reflexum IIndl. $\beta$. Intermedlum Fal.

Forma intermedia quasi inter Oncidium caesium Rehb. fil. et reflexum; pseudobulbis folisque subcaesiis; labelli lobis posticis oblique ovatis subretrorsis, lobo medio reniformi trilobo lateralibus subduplo latiore, lobulis margine interiore imbricatis; callo baseos plurituberculato, antrorsum bicruri; columnae alis dolabriformibus apice acutiusculis.

Sepala et petala reflexa viridi lutea et fusco variegata. Labellum citrinum utrinque ad callum maculis duabus fuscescentibus. (Rgl.).

Oneldium sangulneum Clnd!. $\beta$. roseum Itgl.

Sepalis petalisque albis, roseo-maculatis; labello fusco-luteo et violaceo-variegato; columna rosea, superne utrinque auriculato-biloba. Cetera ut genuinae.

\section{Gneldlum uniforum LIndI. $\beta$. robustum $\mathrm{Egl}$}

Differt foliis floribusque majoribus, pedunculis $2-3$ ftoris, sepalis anguste lanceolatis, basin versus cuneatis, obtusis apice inaequaliter bilobis; petalis brevioribus, ovato-lanceolatis sepalisque brunneomaculatis.

An forma hybrida inter 0 . uniflorum et longipes? (Rgl.).

\section{Lockhartia obtusifolla ingl.}

Foliis triangularibus, obtusis, carinatis; paniculis paucifloris axillaribus; bracteis membranaceis, acutis, labelli hastati lobis lateralibus acutiusculis, intermedio oblongo, obtuso.

Caules graciles, adscendentes, usque pedales. Folia disticha, equitantia, imbricata, elongato-triangularia, obtusa, $3 / 4$ pollicem longa, $1 / 4$ pollicu lata. Paniculae axillares, 2-pluriflorae, inferne bracteis curdatolanceolatis acutis appressis, superne bracteis cordato-subrotundis cucullatis, patentibus, apice mucronulato-acutis. Flos parvus, flavus, vix $1 / 3$ pollicaris in diametro. Sepala 3 recurvo-patentia, ovata, concava, apice apiculata. Petala subfalcato-ovato-oblonga, subcrispa, obtusa, erectopatentia. Labellum hastato-trilobum, brunneo pictum; lobis lateralibus (hasilaribus) lineari-triangularibus, acutis vel obtusiusculis et apice denticulatis; lobo intermedio oblongo apice denticulato; disco tuberculato, tuberculis anticis parvis, posticis maximis. Columnae alae ovatae acutae, superne acute denticulatae Pollinia 2, clavata, pedicellata, glandula parra. Inforescentia et flore Lockhartiae acutae, folio et labio L. eleganti aftinis.

Cl. Lansberg misit plantas vivas e Columbia. (Rgl.). 
Qockhartia parthenocomos uchb. At. $\beta$. crispula agl.

Bothriostigma distichophyllum Hort. Petrop.

Foliis disticbis, elongato-lanceolatis, incurvato-acutis; pedunculis axillaribus, $1-3$ floris, bracteis late cordatis; sepalis ovatis, acutis; petalis sepala paullo superantibus, obovatis, obtusis; labello obtuso-trilobo, crispulo, margine subdenticulato, lobis lateralibus incurvis, lobo intermedio apice subretuso, disco piloso.

Cl. Lansberg e Columbia nis! (Rgl.).

\section{Promenaea Lollinonl a.Indt. $\beta$. obtusa Bal.}

Differt: labelli lobis lateralihus lanceolatis luteo-albis et Iransverse rubro-striatis, lobo intermedio ovato obtuso luteo et immaculato; callo disci postice fornicato triangulari ovato; columna basi macula sanguinea. (Rgl.).

\section{Medychlum pallidum Iregl.}

Glabrum; foliis ublongo-lanceolatis, undulatis, acuminatis; spicis terminalibus laxis paucifloris; bracteis unifloris, tubo $1 / 3$ breyioribus, sparsis, omnibus convolutis, lanceolatis, margine membranacels, apice tantum pilosulis; lloribus solitariis, sparsis, pallide luteis; lacinits limbi obverse lineari - oblongis, obtusiusculis, labellum subaequantibuș; labello cuneato-obovato et apice bipartito. lobis, obtusis, vel diphyllo foliolis obverse lanceolatis apice emarginatis; filamento rubicundo, quam labellum sesquilongiore.

Caulis $3-4$ pedalis. Folia circiter 14 poll. longa et $3 \frac{1}{2}-3 \frac{3}{4}$ poll. lata, supra obscure viridia, punctis raris minutissimis albis adspersa, subtus pallidiora et punctis creberrimis albidis obtecta. Labellum $1 \frac{1}{\mathrm{~s}}$ poll. longum. - Affine Hedychio Gardneriano, quod differt foliis ellipticis, bracteis $1-2$ floris, stamine longissimo et spica magna multiflora, Labello integro vel bidentato.

Patria ignota. (Kgl.).

\section{Medychlum villosum Eoxb. $\beta$. Ianceolatum Isql.}

Foliis lanceolatis, spicis abbréviatis paucifloris. (Rgl.).

\section{Chanaeróps humblis r..}

a. genuina; caudice arborescente; petiolis foliorum a basi ad apicem margine aculeis munitis; foliis rigidis viridibus.

ß. glabrescens Rgl.; caudice basi sobolifero; petiolis foliorum a basi ad apicem margine aculeatis, aculeis supe re parvis, foliis tenuioribus glaucescentibus,

$\gamma$. subinermis Rgl,; caudice basi sobolifero; petiolis foliorum supra medium margine aculeatis, superne inermibus. (Rgl.).

\section{Erousionetla Li azinok! sleb.}

Br. Kaempféri llort. Petrop.

Capitulis masculis axillaribus, solitariis; pedunculis quap̣ petioli duplo longioribus. (Mgl.). 


\section{Telanthera Meyeriana thegel et Mónulcke,}

(Telanthera sect. II. Brandesia ${ }^{\star \star \star}$ ) pedunculi trifidi vel trichotomi. Capitula solitaria vel 2-3-nata, Moq, in DC. prodr. XIII., sect. post. 373). Caule herbaces, inferne patenti-hirsuto, superne appresso-pubescente; foliis ianceolatis, petiolatis, integris, acuminatis, versus basin in petiolum angustatis supra strigilloso-subtus mollius pubescentibus; pedunculis longis, trichotomis, patentibus aut saepe patentissinis vel subdivaricatis, rectis; capitulis demum oblongis, obtusis, aphyllis; floribus albis, opatis; sepalis quam bracteae subtiliter acuminatae subtriplo longioribus, ob. longis, acutis, glabris. - In horto altitudinem $2_{2}^{1}$ pedium assequitur. Caulis laxus, feres, inferne purpurascens, supra genicula inflatus, 1 linea in diametro paullo erassior. Folia cum petiolo usque $3 \frac{1}{2}$ pollicem longa et usque $1 \frac{1}{*}$ pollicem lata, superiora minora, laete vipidia, cum nervis concoloria, petiolo $1 / 2-3 / 4$ pollicari. Pedunculi laterales usque 4 pollicares, medius plerumque brevior interdum ad nihil redactus, omnes appresso-pubescentes. Capitula singula vel 2-3-nati, primum globosa demum oblonga, usque 10 lineas longa, plerumque breviora, 3 lineas lata, obtusa, in specimjuibus exsiccatis interdum acutiuscula. Bracteas lanceolatae, in aristam acuminatae, laterales in nervo medio infra apicem villosae, tertia glabra, tenui-membranaceae, omnes subaequales. Flores 2 lineas longi. Sepala uninervia, nervo vix prominente. Stamina calyce breviora; tubus vix viridescens. Staminodia antherarum apicem superantia, in apice 3-4 dentato-laciniata, in margine integra, Antherae lineares, flavae. Stylus breviusculus. Stigma globoso-capitatum, - Pili in omuibus partibus persistentes.

Prope Cochabamba Junjo 1848 leg, Cuming. (Kcke.).

\section{Oreodaphne regalls mgl. (Sectio 4 Umbellaria Nees).}

Arbor glaherrima; ramis teretibus laevibus, junioribus viridibus: gemmis axillaribus: foliis sparsis, petiolatis, oblongo-lanceolatis, basi rotundatis, apice obtusiusculis, penninerviis, costa media valida percursis, in utraque pagina reti ulato-venosis, usque 4 pollices longis et 1 pollicem latis; petiolis circiter 3 lineas longis; umbellis axillaribus, 7 foris; pedunculo communi longitudine petioli ; involucri foliolis membranaceis, venis longitudinaliter nervosis, caducis, glabris; pedicellis $3 / 4$ pollicem longis, apicem versus incrassatis, perianthio 6 -partito, infundibuliformi-campanulato: laciniis lanceolatis, concavis, obtusic. $1 \frac{1}{2}$ lineam longis, pellucido-punctatis; staminibus 9 , aequalibus, perianthio paullo brevioribus, tribus interioribus basi glandulis duabus geminatis, antheris oblongis quadrilocellatis, valvato-dehiscentibus; staminodiis tribus, quarti ordinis, vix tertiam partem filamentorum aequantibus; ovario ovatoLurbinato, stylo stamina subaequante coronato.

Ddor foliorum et ramorum pulchre aromaticus, (Bgl.).

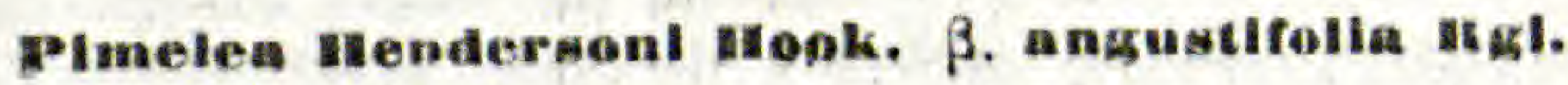

Poliís confertis, anguste lineari-lanceolatis, (Rgl.).

\section{Gnidla janiperifolla dam. $\gamma$, aurea Eql.}

Foliis subtrinervis, capitulis plurifloris, flopibus involucrum superantibus. G. aurea Hort. (Rgl.). 
Wakea Tehamanlana Metan.

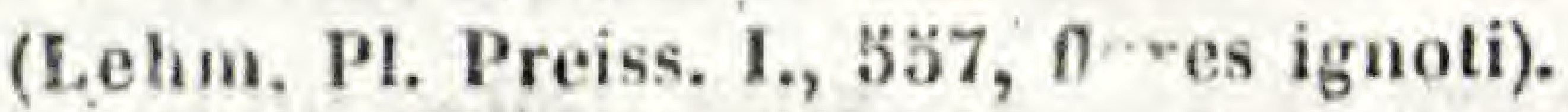

Racemis ahbreviatis glomeruliformihus axillaribus basi bracteis squamiformibus minutis suffultis; floribus lavis; pedicellis pubescentihus; sepalis linearibus revolutis; stylo adscendente, stigmate capitato, oblique truncato et supra basin tuberculo conoideo. ( $\mathrm{R} g \mathrm{l}$. .).

Rerekilnia ragl. (Nov, gen. Proteacearum).

Perigonium tetraphyllum, fóliolis linearibus, apice subcochleariformibus, patentibus vel subrevolutis. Filamenta 4 vel sub apice sepalis aduata vel plus minus libera. Antherae liberae. Glandula unica perigyna ovario adnata, caduca. Ovarium sessile biovulatum. Stylus elongatus, rectus vel declinatus. Stigma conicum. Fructus ...... Folia teretifiliformia, pinnati-partita. Racemi corymbosi, terminales, basi bracteis deciduis suffulti.

\section{Mercklluia rosea ugl.}

Ramis brevibus confertis; folits tereti-filiformibus trifidis vel rarius pinnatim $4-5$ fidis, scabriuseulis, junioribus ramulisque hirsutis, vix ad $3 / 4$ pollicem longis, segmentis indivisis spinescentibus petioloque exsulcis, usque $1 / 3$ poll. longis; corymbis terminalibus, subsessilibus, abbreviatis; involucro membranaceo imbriı ato glabro caduco, rhachi villosa; calycibus pedicellisque tiliformibus, glabris, roseis, diu clausis limbo vix inflato, apertis sepalis linearibus dbtusis apice parum latioribus; filamentis liberis vel cum sepalis plus minusve conuatis; antheris liberis; stylo erecto vel declinato.

Hakea lissocarpa R. Br., cui proxime, accedit, differt: ramulis elongatis; foliis plerumque pinnatim 5 -fidis, junioribus ramulisque pubescentibus, usque $1^{1} / 2$ poll. longis; petiolo supra deplanato unisulco; foliorum segmentis usque $3 / 4$ poll. Iongis; racemis axillaribus folio brevioribus; calycibus diu clausis, limbo globoso-inflato, apertis sepalis filiformibus superne cochleariformi-rotundis; filamentis cum sepalis connatis; antheris sepalorum apici immersis. (Rgl.).

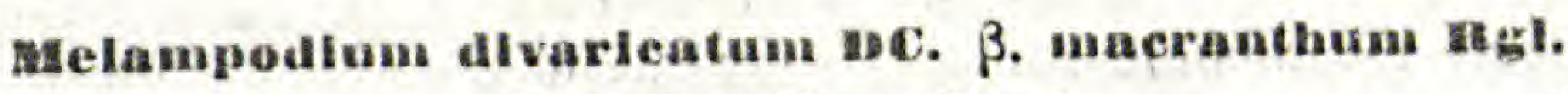

M. macrauthum Hort.

\section{;}

Differt a planta genuina radiis'subduplo longioribus. Semina misit de Warscewicz anno 1816 ad hort. bot. Turic. a Guatemala. (Rigl.).

seneclo aeyeri aql. (DC. prodr. VI, 370, ser. IV. Discoidei).

Suffruticosus, glaber, glaucus, erectus; foliis sessilibus, coriaceis, ovato-oblongis vel superioribus lanceolatis, basi plus minus auriculatohastatis, apice mucronato-acutis, margine irregulariter acuteque denticulatis; corymbo composito terminali; involucro cylindrico quam discus quartam partem breviore, 8-phyllo, phyllis margine membranaceis apice pilosulis obtusiusculis; bracteolis $4-5$, lineari-lanceolatis, apice sphacelatis; floribus $12-15$, tubulosis; acheniis sulcatis hispidulis.

Senecio odoratus, ei proximus, djlfert folis angustioribus (non glaucis?); involucri squamis quam discus dimidio brevioribus, apice mucronato-acuminatis; bracteolis $2-3$, floribus $8-12$; achaenis pilosulis.

Semina misit Ferd. Müller ex Nova Hollandia. (Rgl.). 


\section{Calyptrostigma Trautv, et Mey.}

Calycis tubus cum ovario connatus, oblongus, apice in collum angustatus; limbo supero quinquefido, laciniis inaequalibus, lineari-lanceolatis, reflexis, inferioribus 2 basi tantum, superioribus 3 ad medium vel usque versus apicem connatis. Corolla supera, tubulosa, e basi angusta intlata; limbi quinquefldi subbilobi laciniis obtusis. Stamina $\breve{3}$ basi corollae adnata, inter se libera, corolla breviora. Antherae lineares, inter se et cum styli apice connatae. Glandula epigyna 1. Ovarium inferum, biloculare. Stylus filiformis, stigmate peltato calyptriformi, Ovula in loculis numerosa, pendula.

\section{Calyptrostigma MIddenderflana Trautv. et Mey.}

(Weigelia Middendorffiana Fisch. Diervilla Middendorffiana Carr.).

Frutex humilis; ramulis utrinque uniserialiter pilosis, deinde glabris; foliis oppositis, subsessilibus, ovato-lanceolatis, subplicatis, acutiusculis, serratis, piloso-ciliatis, utrinque glabris; floribus in corymbos 3-4 flores terminales vel axillares congestis, pedicellatis, folio plerumque brevioribus, pedicellis bracteis linearibus munitis.

Calycis laciniae lineari-lanceolatae vel lanceolatae bracteisque margine ciliatae, corollae tubo triplo breviores. Stamina basi pilosa. Flores citrini, intus croceo-punctatae vel pulchre rubri, $1 \frac{1}{3}$ poll longi, limbo vix pollicem in diametro tendente. Folia usque 3 pollices longa et $1 \frac{1}{4}$ poll. in diametro lata, laete viridia. Hab. in Sibiria orientali. (Kgl.).

\section{Jasninum tenulfollum IRgl.}

Jasminum Bidwillianum Hort. Petersb. (Jasminum DC. prodr. VIII., 1*, calyc. lobis subulatis).

Fruticosum; ramis gracilibus, dependentibus, teretibus, subasperulis; foliis simplicibus, oppositis vel suboppositis, breviter petiolatis, lineari-lanceolatis vel linearibus, acuminatis, trinerviis, glabris, usque 2 pollices longis, $1-3$ lineas latis, petiolo articulato hispidulo; corymbis 3-plurifloris, terminalibu; calycis lobis plerumque 6, subulatis, margine glandulosis, tubum aequantibus; corolla alba, limbo 8-lobo, lobis linearibus acutis.

Corollae tubus gracilis, $1 / 3$ pollicem longus, limbus $3 / 4$ poll. latus. Flores suaveolentes.

Patria ignota. (Rgl.).

\section{Cenolobus mollis RgI.}

Tota planta pilis patentibus molliter pilosa; caule volnbili; foliis ovatis, basi truncato-rotundatis vel vix cordatis, apice abrupte acuminatis vel rarius obtusis, pedunculis plurifloris folium subaequantibus, glomerato-racemosis; sepalis lanceolato-subulatis, hispidis; petalis ovatis, apicem versus attenuatis et obtusis, utrinque breviter pilosis, extus sub apice vix barbulatis, dein patulis.

Folia opposita, petiolata, 2 poll. longa, $1 \frac{1}{2}$ poll. lata. Flores parvi, virides, $2-3$ lineas in diametro tendentes.

Patria Brasilia. (Rgl.). 
Negeta жrandifiora v.

Certissime varietas tantum Népetae grandiflorae; differt caule puberulo, foliis supra viridibus vix puberulis, subtus pallidis canescentibus, calycibus pubescentibus.

A Nepeta racemosa $L a m$. differt planta nostra racemo ramoso, cymis pedunculatis usque 20 floris, corollis calyce vix duplo longiovibus. Sub nomine Nepetae Reichenbachianae Fisch, et Mey, in horto Petrop. culta, a cl. Ledebour Benthamium secuto ad $\mathrm{N}$. racemosam Lam. ducta. (Rgl.).

\section{Citharexylon tomențosum Kith. $\beta$. molle IRgl.}

Tota planta pilis simplicibus molliter pilosa; foliis ovatis vel rhomboideo-ovatis, acutis, obtusjs vel retusis, integerrimis vel grosse crenatis, usque $1 \frac{1}{2}$ poll. longis et $1 \frac{1}{4}$ poll. latis. Reliqua ut genuinae. An spec. propria? (Rgl.).

\section{Clerodendron Lindleyi Dene.}

Cl. fragrans fl. simplici Lindl. Bot. Reg. 24, t. 41. Cl. foetidum Hort. Paris. Cl. Lindleyi Dcne. Fl. des serres 9, 17. (Rgl.).

\section{Ipomoes tuhèrculata.}

Sub nomine Ipomoeae tuberculatae a cl. Choisy species plures bene distinctae conjunguntur; scilicet:

\section{1) Epomoea tuberculata Desrouss.}

Caule sarmentoso, 2-3-pedali (in planta nostra $10-15$ pedali); foliis palmato-septempartitis, lobis lanceolatis vel lineari-lanceolatis, integris mucronatis; petiolis tuberculato-asperis; pedunculis $1-3$ floris; sepalis obtusis, inaequalibus, ovatis, margine scariosis; corolla rosea speciosa.

Ipomoea digitata probabiliter ad hane speciem pertinet.

Insula Bourbon.

ß. angustifolia Rgl.; glabra; caule volubili laevi; foliis digitatoseptempartitis; lobis lineari-lanceolatis vel lanceolatis, obtusiusculis vel subretusis vel sub apice in aristam desinentibus, margine integerrimis vel repandis; petiolis plus minus tuberculato-asperis, basi nudis; pedunculis medio bibracteatis, $1-3$ floris, folium subaequantibus vel eo brevioribus; sepalis ovatis, obtusissimis, inaequalibus; corolla rosea, speciosa.

Radix tuberosa. Caulis rolubilis usque $10-15$ pedalis. Pedunculus $1^{1} / 2$ pollicaris. Foliorum lobi usque $2^{1 / 2}$ poll. Iongi et $1 / 4$ poll. lati. Sepala $1 / 4-1 / 3$ poll. longa.

Colitur in horto Petrop. sub nomine falso J. Willdenowii.

\section{2) Промоoеa daequini IRgl.}

I. stipulacea Jacq. Hort. Schoenbr. tab. 199.

Caule volubili duodecim pedali; foliis palmato-quinquepartitis; lobis lanceolatis acutis integerrimis; petiolo cauleque laevi; stipulis geminis palmatis; pedunculis Irifloris; sepalis, ovatis, acutis; corolla lilacina. 


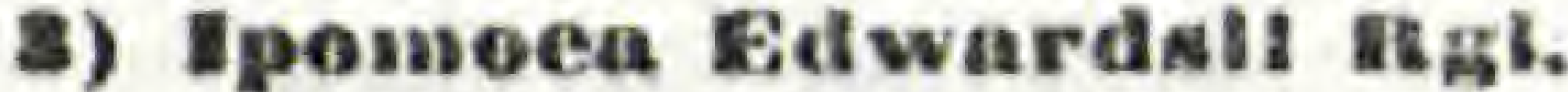

I. tuberculata Edw. in Bot. Reg. tab. 86 .

Ramis tubereulatis; foliis palmato-quinquepartitis; foliolis ovalilanceolatis, mucronulatis, extimis duobus integris rel bipartilis; petiolo lacvi vel tuberculato; stipulis nullis; pedunculis axillaribus, 3 -floris; sepalis 3 exterioribus cordatis, interioribus 2 sublongioribus tenuioribus acutioribus; corolla sulphurea.

Insulae Capitis virids. (Rgl.).

Cyrianthera Pohllana $\mathbf{~}$, ab E. $\gamma$. dlscolor wC. prodr. XI., 330.

C. magnifica Hort.

Foliis subtus bracteisque purpureis; bracteis puberulis ciliatis, obtusissimis. (Rgl.).

\section{Cyclamen Coum Mill. $\gamma$. pulcherrlmum ral. Cyclamen vernum Sweet.}

Tuber disciformis. Folia reniformia, cordato-subrotunda, obscuriuscula, margine exangulata vix crenulata, supra viridia et albo-maculosa, subtus purpurea. Calycis laciniae lineari-lanceolatae. Corolla tubo urceolato, ad limbum constricto; fauce subpentagona, lobis oblongis, contortis, apice denticulatis, pulcherrime carmineis et basi maculo atropurpureo ornatis. - Varietas pulcherrimà.

Tubera misit Hartwiss e Caucaso? (Rgl.).

\section{Epacris IIookeri regl.}

E. obtusifolia Hook. Bot. Mag. tab. 377ä, nec Sm.

Ramulis junioribus pubescentibus deinde glabris; foliis breviter petiolatis, anguste lanceolatis, erecto patentibus, concaviusculis, callosoobtusiusculis, margine integris, subtus trinerviis; floribus axillaribus breviter pedunculatis, in racemum secundum dispositis, patulis vel pendulis; calveibus lanceolatis, obtusis, tentiter viliatis, corollae tubo daplo brevioribus; corollis urceolato-tubulosis, latiludine duplo longioribus, albis; staminibus inclusis; stylo glabro. Floret A prili.

B. obtusifolia Smith (Exotic Botany tab, 40) differt foliis 5-nerviis, calycibus corollae tubo aequalibus; corollis campanulatis, brevioribus, aequilongis ac latis. - Floret Julio.

Patria Süd-Wales. (Rgl.).

\section{Brica arborea l.. $\beta$. asturea wort. Petrop.}

Corollis parvis, depresso-subglobosis, latioribus quam longis, vix $1 / 2$ lineam longis. (Rgl.).

\section{Frlea Burchell Egl.}

E. pellucida Andr. є. brevillora Benth. in DC. Prodr. III., 632. - E. Meuroni palliditlora Hort. Petrop. (9, Tubillorae. d, Autherae muticae, bracteae calyci approximatae. Capsula 4-locularis. Folia quaterna. Rgl. Eric. pag, 86).

Foliis anguste linearibus; calycibus floribusque pilis glanduliferis hirlis: floribus $2-8-n$ is, pedicellatis; pedicellis calycem aeqquantibus; stylo exserto. 
Frulex humilis, $2-3$ ptdalis. Nami stricti, hirsuti et glandulosopilosi. Folia ıuaterna, erecto-patentia, deinde patentia, anguste linearia, margine revoluta, dorso sulcata, acuta, pilis latitudine foliorum hrevioribus et apice glanduliferis hirta, vix $1^{1 / 2}-2$ lineas longa. Pedicelli cabycem aequantes, Flores terminales, 1-7. Sepala e basi latiore linearia, acuta, foliacea, apicem versus margine-avoluta, pilis glauduliferis hirta, $3 / 4$ lineam longa. Corolla tubulosa, vix incurva, costata, apicem versus paullo inflati, sub fauce constricta, vix $1 / 2$ pollicem longa, $1 / 1 / 2-2$ lineas Iata; lobis obtusissimis, patentibus. Antherae inclusae, muticae, fuscoatrae, filanento apice recurvato lateraliter insidentes. Stylus exsertus. Affinis E. exsudanti el Reineckeanae. Differt foliis linearibus brevioribus erecto-patentibus pilis glanduliferis hirsutis; floribus brevioribus vix incurvis, pilis glanduliferis adspersis, vix $1 / 2$ pollicem longis; stylo exserto.

Patria: Caput bonae spei. (Kgl.).

\section{Erlea fitifolla mgl.}

(Sect. XVII. Dasyanthes DC. prodr. VII., 636).

Foliis 8-nis, laxe patentibus, filiformibus, pubescentibus, acutis; foribus in apice ramulorum lateralium solitariis, sepalis e basi latiore subulatis, margine glandulosis; corollis tubulosis, cylindricis, puberulis, limbo brevi recto; antheris jnclusis muticis; ovario depresso-globoso, villoso.

Virgato-ramosa, ramis glabriusculis. Folia laete viridia usque $1 / 2$ poll. longa. Ramuli laterales floriferi, breves $1 / 4-2$ poll. longi, Calyx tertiam partem corollae aequans. Corolla recta, pollicem longa, basi albida, apicem versus rosea. An planta hybrida?

Patria ignota. (Rgl.). ;

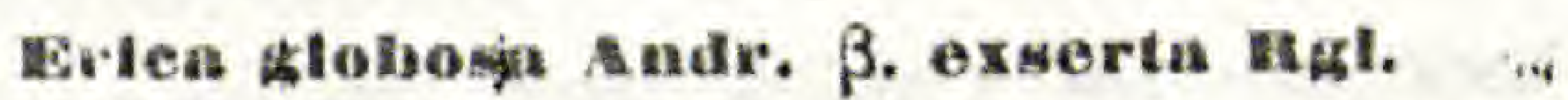

Foliis quaternis, antheris exsertis. Reliqua ut genuinae. (Rgl.).

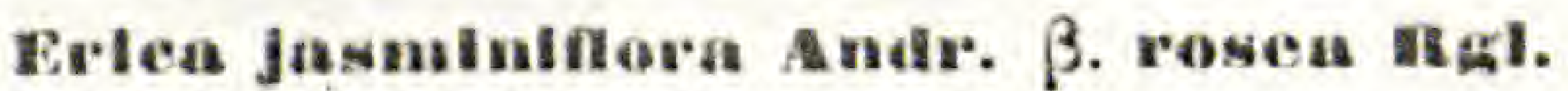

Differt: sepalis lanceolatis, limbi lobis roseis. An planta hybrida cum E. Shannoniana? (Rgl.).

\section{Crica mieroealyx Itgl.}

(Sect. 32 Cupressinae Rgl. Anactasis Benth.).

Frutex $1-3$ pedalis; caule foliisque hirsutis; foliis breviter petiolatis, incurvato-patentibus, quaternis, lineari-lanceolatis, margine revolutis, supra hirsutis laete viridibus, subtus albidis; floribus 1-4-nis, in apice ramorum subsessilibus, calyce minnio, corollae appresso, sepalis subrotundo-ovatis, ciliatis, roseis, apice muticis vel viridi-apiculatis; corollis globoso-urceolatis, glabris, roseis, limbi laciniis erectis, basi angulo ac.to; antheris inclusis, brunneis, breviter aristatis, aristis sub lente hirsutis.

Affinis Ericae Bergianae et probabiliter planta hybrida. Folia et flores circiter $1 \frac{1}{2}$ lin. longi. ( $\mathrm{Kgl}$.).

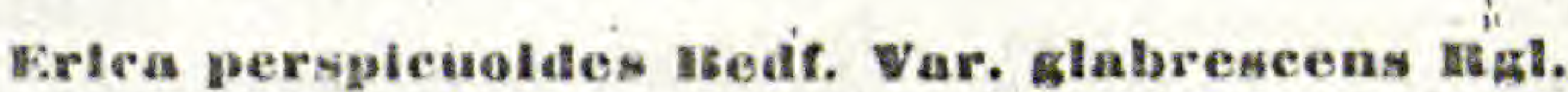

Foliis virescentibus, junioribus ciliatis deinde glabris; Loribus pulchre incarnato-roseis. (Rgl.). . 


\section{$\rightarrow 0$ \\ Erlca Dachll igh.}

(Minutiflorae Rgl.; Chlorocodon Bth. Differt a sectione bracteis oppositis, sepalis corollam aequantibus; antheris aristatis).

Ramis laxis, junioribus puberulis; foliis quaternis incurvo-erectopatentibus, linearibus, acutis, dorso sulcatis, glabris; floribus sub apice ramorum verticillatis; petiolis gracilibus, puberulis, basin versus bracteis 2 oppositis linearibus munitis, $2 \frac{1}{2}$ lineas longis; sepalis anguste lanceolatis, viridibus, corollam aequantibus, margine puberulis; corolla late campanulata, 1/4 lineam longa, lobis limbi latis incurvis, carnea et apice rubella; antheris inclusis, late cristato-aristatis, fuscis; stigmate peltato, subexserto. Patria ignota. (Rgl.).

Eriea rubrecalyx Andr. $\beta$. tenulfora Iggl.

Differt corolla tubulosa (nec inflata), 1/3-1/2 poll. longa, $1 \frac{1}{2} \mathrm{lin}$. lata, alba, limbo erecto-patente concolore. (Rgl.).

Erica vestltoldes igl. (E. fulgens Hort. Petrop.).

Foliis senis, lineari-filiformibus, acutis, supra brevissime pilosis, erectis; floribus infra apices ramorum verticillato-racemosis; bracteis approximatis linearibus vel lanceolatis, margine glandulosis; sepalis herbaceis, e basi ovata longe acuminatis, margine glandulosis, corolla triplo brevioribus; corollis rectis, tubulosis ( $\% / 8$ poll. longis), subcostatis, purpureis, extus pilosulis, supra medium inflatis, fauce constricto, laciniis obtusis, recurvato-patentibus, crispatis, crenulatis; antheris muticis, inclusis; germine villoso.

B. echiiflorae proxima. (Rgl.).

\section{Erlea Iftulthora Salob. $\gamma$. brevifora mgl.} (Rgl.).

Floribus subsessilibus, corollis calycem vix sesqui superantibus.

Aretostaphylos mucronlfera ic. prodr. VII., 2, 283, no. 9.

(Flores ignoti).

Paniculis racemosis terminalibus, ramis paucis nutantibus; bracteis nembranaceis, petiolo brevioribus; calycis lobis ovatis, obtusiusculis, margine membranaceis et deinde laceris; corollis ovoideo-urceolatis, faucem versus conoideo-attenuatis, ex albido viridibus, fauce brevi 5-partito, lobis latissimis deinde recurvatis crenatis; staminibus villosis.

Comarostaphylis arbutoides Lindl. cui proxime accedit, differt: bracteis foliaceis, superioribus pedunculo florisque gemma longioribus, ciliatis; calycis lobis lanceolatis, acutis; floribus albis subduplo majoribus et globoso-urceolatis, staminibus? (Kgl.).

\section{Gaultherla Kowil Ingl.}

Brecta; ramis petiolis pedunculisque setoso-pilosis; foliis breviter petiolatis, ovato-oblongis, utrinque strigoso-pilosis, apice calloso-apiculatis, margine crenulatis et crenulis seta terminatis; racemis terminaIi. is; pedicellis basi tribracteatis; bracteis cucullato-ovatis, margine tantum ciliatis, pedicellis multo brevioribus; corollis urceolato-conoideis, angulatis, puberulis; antheris 4-aristatis. 
Affinis Gaultheriaé strigosae Bienth., inflorescentia briácteis et antherarum aristis dignoscitur. Frutex $2-3$-pedalis, pilis selosis deinde nigricantibus plerumque patentibus hispidus. Folia usque $2 \frac{1}{2}$ pollices longa et $1^{1} / 4$ poil. lata, obscdre viridia. Racemi subnutantes pluriflori. Calyx tubo hispido, dentibus lanceolatis glabriusculis vel ciliatis. Corolla $1 / 3$ pollicem longa, pulchre rosea.

Accepimus nomine Gaultheriae coccineae ex horto Lowii. (Rgl.).

\section{Arbutas vaedo $\mathrm{L}$. War. sallelfolla ingl.}

Caule fruticoso humiliori; foliis oblongo-lanceolatis, serratis. (Rgl.). Dimetopla Sisocarpa Barti. Ind. sem, Goett. 1845.

Mericarpio utroque muricato. (Rgl.).

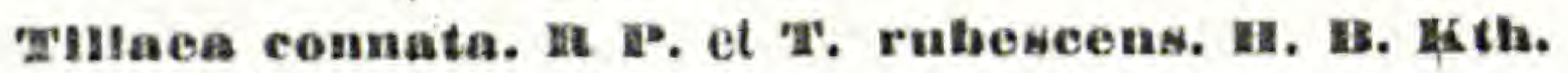

quas cl. Candolle conjunxit, dignoscuntur:

a. T. rubescens: caule pygmaeo; foliis inferioribus basi connatis, superioribus sessilibus; calyçibus fructiferis globosis.

b. $T$. connata: caule elatiore; foliis omnibus connatis; calycibus fructiferis ovato-oblongis. ( $\mathrm{Rgl}$.).

\section{Bedum Sieboldil Mort.}

Glaberrimum; radice perenni carnosa; caulibus decumbentibus rubicundis; foliis crassis, playis, ternatis, glaucescentibus, subsessilibus, e basi cuneata integerrima subrotupdis, apice repando-crenatis et obtusissimis; cymis terminalibus multifloris; bracteis ovato cuneatis; bracteolis oblongo-ellipticis; calycis dentibus acutis, corolla triplo brevioribus; petalis elliptico-lanceolatis, acutis, roseis; staminibus 10, petala superantibus.

Caules usque pedales. Folia deinde rubro-marginata vel rubentia, $1 \% / 4$ poll. longa et lata, - S. rubro Edg. affine.

E Japonia, adhuc indesçripta.; (Rgl.).

\section{Henchera obtusissima Kgl. et Hach.}

H. americana et H. lucida Hort.

Petiolis paniculisque strictis pilis breviusculis hispidis, basi glabriusculis; foliis hispidulis, late ovatis, profunde coriatis, leviter lobatis, lobis basilaribus late imbricantibus, terminali latitudine sua duplo breviore, dentibus truncatis mucronulatis; calycis dentibus aequalibus, brevihus, erectis, obtusissimis, interstitiis latissimis rotundatis; petalis cuneatis obtusis, exunguiculatis, calyce brevioribus; staminibus quam calycis dentes subtriplo longioribus; stylis crassis stamina demum superantibus. Am. bor.?

Robusta, $1^{1} / 2-2$ pedalis. Folia opaca. Calycis dentes $1^{1 / 2}$ lin. lati vix 2 lin. longi. - Species habitu stricto et calycis forma distinctissima.

a. glabrior. Petiolis fóliisque glabriusculis; calycibus longioribus; petalis calycem demum superantibus; stylis gracilioribus stamina vix aequantibus.

H. americana var, oblusiloba Hort. (Hch.). 
Willeuchera undulata Figl. et Inach.

H. americana, H. divaricata, H. hispida et H. Menziesil Hort.

Paniculis calyeibusque pilis brevibus glandulosis dense hirsutis; petiolis pilis brevissimis glandulosis adspersis; foliis ovatis, rarius subrotundis, profunde cordatis, undulatis, hispido-ciliatis, quinquelobis, lobis approximatis, basilaribus saepe imbricantibus; terminalibus subcuneatis, acutis grosse dentatis, dentibus acuminatu-mucronatis; calycibus campanulatis, basi breve obconicis, dentibus inaequalibus obtusis, rarius mucronulatis; petalis plus minusve lineari-spathulatis acutis, rarius obtusis, ex iguiculatis, apice ciliolatis et saepe irregulariter crenulatis, omnibus dentes calycinos superiores aequantibus; staminibus stylisque ifuam dentes calycini demum duplo longioribus. - $\mathrm{Am}$. bor.?

Gracilis, $1 \frac{1}{2}$ pedalis. Folia opaca, laete viridia, 2 poll, longa, 14 lin. lata. Paniculae lasae, $1-3$ foliatae vel subnudae. Pedunculi et pedicelli filiformes. Flores 2 lin. longi, 1 lin. lati. (Reh.).

\section{weuchera anerienna $\mathrm{L}$.}

Hujus speciei varietates sequentes forsan hybridas vel ex hybridis ortas colimus:

a, robusta. $3-4$ pedalis. Petiolis pedunculisque hispidis, basi pilis brevissimis glandulosis scabro-puberuli ; folis nitidulis, maximis, rotundatis, profunde cordatis lobatisve, dentibus obtusis mur ronatis; paniculis amplis, pilis glandislosis dense pubescentibus; calycibus $1 \frac{1}{2}$ lin. latis, 2 lin. longis; petalis vix coloratis; staminibus calycis dentes demum 2-3-plo superantibus.

H. americana et $\mathbf{H}$. hispida Hort.

$\beta$. gracilior. $2-2^{1} / 2$ pedalis. Petiolis pedunculisque indumento var. $\alpha$. sed multo breviore et molliore obtectis; foliis nitidis mediocribus minus profunde cordatis lobatisve, lobis saepe acutis, dentibus aculiusculis et subacuminatis; calycibus 1 lin. latis vix $1 \frac{1}{2}$ lin. longis purpurascentibus; staminibus calycis dentes demum subtriplo excedentibus.

H. macrophylla et H. americana Hort.

$\gamma$. affinis. Habitus, folia et flores fere H. obtusissimae, sed gracilior et minus stricta, indumento molliore, foliorum lobis basilaribus divergentibus, rarissime approximatis et imbricatis, dentibus obtusis floribusque longioribus.

H, divaricata et H. macrophylla Hort.

ס. lara, (iracilis, 1-2 pedalis. Petiolis pedunculisque dense pubescentibus vel hispidiusculis, basi glabrescentibus; foliis opacis vel uitidulis rotundatis, profunde cordatis, lobis obtusis, dentibus obtusinsculis mucronulatis; paniculis laxis, foribus praecedentis, stylis brevioribus tenuioribus.

H. americana, H. micrantha et $\mathbf{H}$. hispida Hort.

ع. minima. Omnia var. 8., sed vix pedalis, paniculis folia parum superantibus, foribus minoribus vix $1 \mathrm{lin}$. latis et $1 \frac{1}{2} \mathrm{lin}$. Iongis.

H. americana Hort. (Rch.).

\section{Aqullegia advena Hort. Petrop.}

Calcaribus apice vix incurvis, lamina obtusa longioribus, rarius calcaribus nullis; sepalis acutiusculls, apice calloso viridibus, quam petala subduplo longioribus, coeruleis superne non albis. (Kgl.). 
Aquilegia whiriea I.ins. Var. Ntenosepala Bat.

Floribus pallide coeruleis; sepalis lanceolatis; pelalis lamina subtriplo longioribus. ( $\mathrm{Rgl}$.).

\section{Aqullegia volgaris d.' A. Kareliniana Hort. Petrop.}

ß. caucasica Ledebour. Differt caule viscoso; sepalis petalorum lamina longioribus, acuminatis; petalorum laniua ohtusa nee relusa.

$\gamma$, viscosa Rgl. Caule viscoso; flore atrocoeruleo; sepalis petalorum laminam subaequantibus. ( $\mathrm{K} g \mathrm{l}$.). ,

\section{Eplonedium rubrum Eart. Angl.}

(An E. rubrum Morr. Journ. d'Hort. 1844?).

Caule subpedali pilosiusculo; foliis triternatis, foliolis cordato-vel subhastato-oblongo-ovatis, acuminatis, rubro-margiuatis et spinulosodentatis; panicula terminali, simplicster ramosa, bracteis minutis, acutis; sepalis 4 concavis, deciduis, rubescentibus, hinis linearibus, binis late ovatis obtusis; corolla 8-petala ; petalis calycis foliolis biseriatim,oppositis; petalis exterioribus ovato-oblon:is, acutiusculis, dorso subcarinatis, pulcherrime carmineis; petalis interioribus cucullatis, in calcar petala exteriora subaequans productis, llavescentibus. ( $\mathrm{Kgl}$.)

\section{Papaver setigerum घoc, $\beta$, nuticun $\mathrm{Rg}$.}

Differt caule glabro vel setiș raris adsperso; foliorum dentibus.muticis v. terminalibus interdum seta terminatis. Forma intermedia inter P. setigeram et somniferum. ( $\mid\langle g|$.$) .$

\section{Cochlearia officinalis t. $\beta$. minor $\mathrm{mgl}$. \\ C. danica Hort. Genev.}

Differt foliis minoribus, caulinis sessilibus, basin versus attenuatis, basi sagittatis.

A C. anglica siliculis duplot ninoribus, foliis caulinis sessilibus; a C. danica siliculis pedunculo duplo brevioribus, foliis caulinis basi attenuatis diversa. $\left(\mathbf{R}_{\mathrm{r}} \mathbf{l}\right.$.).

\section{Maleoluin Ihxa De.}

ß. stenopetala $\mathrm{Rg}$. Malcolmia stenopetala Bernh. certissime forma tantum M. laxae foliis hispidulis petalis angustioribus, siliquis hispidis est. Siliquae plantarum in horto Petropolitano cultarum glabrae vel apice tantum hispidae et pelala albida linearia vel apice latiora el rosea.

$\gamma$. hispidula $\mathrm{Rgl}$. Differt tantum folis pilis raris simplicibus bitidis vel tritidis adspersis. (Rgl:).

Est L. capense Thhg.

\section{Lephaliun, „Ecklonl behrad.}

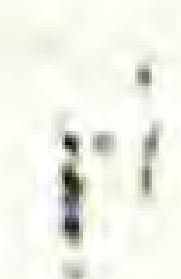

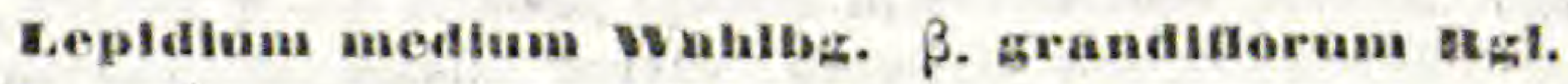

Differt pedunculis longioribus, deinde pollicaribus; caule divarieato, ramoso, glabriusculo, apice calycibusque glanduloso-pilosis. (Rgl.). 


\section{Eraseica oxyrobyncha Coss.}

Planta nostra differt foliis runcinato-pinnatifidis, lobis plerumque subtriangularibus rarius angustioribus. Reliqua ut in descriptione autoris. (Rgl.).

Sinapls abyssinlea A. Eraun.

Melanosinapis abyssinica Hort. Brassica carinata Hort.

Sinapi integrifoliae proxime affinis, differt foliis longe petiolatis, petiolis folio dimidio longioribus, siliquis junioribus rubro-violaceis deinde viridibus, (Rgl.).

\section{Slnapls funcea t. Var. pllosn agl. \\ Sinapis pekinensis Hort.}

Foliis sparse pilosis; foliis superioribus supremisque dentatis. (Rgl.).

\section{Wellophlla Iongifolla bC. $\alpha$. Elabra IEgl.}

Differt pedicellis floriferis glabris. (Rgl.).

\section{Viola tricolor $\mathbf{x}$.}

๔. vulgaris Koch. V. vivariensis Jord. V. Lejeunii Hort. V. macedoaica Boiss.

ß. arvensis Koch. V. agrestis Jord, V. pallescens Jord.

\%. saxatilis Koch. V. Lavida Jord. (Rgl.).

\section{Eehinecaetus hamulesus $\mathrm{Bgl}$.}

Echinocactus 3, Uncinati ${ }^{\star}$ Hamati (Salm. Cacteae in Hort. Dyck.).

Oblongo-cylindricus, laete viridis, 13-angulatus; costis acutis crispis et repando-crenatis; areolis in tuberculis prominentibus, albido-lanatis; aculeis 17-20, externis $16-19$ stellato-radiantibus albidis vel fuscescentibus, centrali unico erecto fusco apice uncinato; flore flavo.

Aculei laeves radiantes tenues circiter $1 / 3$ poll.; aculeus centralis paullo longior et rigidior $1 / 2$ pollicaris, apice uncinato plerumque deorsum verso. Flores mediocres, phyllis sepalinis ovato-oblongis, acutis, membranaceo-narginatis, dorso viridibus; phyllis petalinis lanceolatis, apice eroso denticulatis, citrinis et basi rubris, vix pollicem longis. Stamina numerosa, filamentis basi rubicundis. Stigmata 9 erecta, recurvopatentia.

Semina misit Karwinski. (Rgl.).

Begonia sitlda Dryand. $\beta$. speelosa Mgl.

Differt foliis dimidiato-subcordato-ovatis, duplicato- et angulatodentatis a planta genuina, quae foliis dimidiato-cordato-ovatis oblongis margine subangulatis et obsolete denticulatis gaudet. (Rgl.).

\section{Leplgenum eapense Sehrad.}

Glanduloso-pubescens; caulibus internodiis teretiusculis; foliis linearibus, mucronatis; cymis inferne dichotomis; sepalis anguste lanceolatis, obtusis, violaceo-marginatis; petalis albis subbrevioribus; capsula - ? semina - ? ( $\quad$ (Rgl.). 
I.epigonum diandrum figl. $\beta$. viscldum $\mathbf{m g}$.

Differt ab Arenaria diandra (Guss, prodr. 1. 515) viscositate totius plantae. (Rgl.).

\section{ע.eplgonum glandalosum $\mathrm{x}$. et M. in Mort. Petrö́p.}

Glanduloso-pubescens; caulibus erectis vel adscendentibus; foliis lineari-filiformibus, supra planiuseulis subtus convexis, nucronatis, quam internodia teretiuscula longioribus; ranis racemosis; pedunculis floriferis erectis vel suberectis, sepalis lanceolatis, obtusis, margine scariosis, apice violaceis, capsulam magnam ovatam superantibus; petalis (albis) calyce brevioribus; seminibus ymuibus late marginatis. (R $\mathbf{R}_{\mathrm{b}} \mathbf{l}$ ).

\section{Eepigoun macrothecum FIseh., Mey.}

Inferne glabrum, superne pubescens; internodiis superioribus compressis; stipulis scariosis, ovatis, longe acuminatis, foliis lineari-subalatis, senicylindricis, mucronatis, quam internodia longioribus; ramis racemosis; sepalis lanceolatis, obtusiusculis, margine scariosis, corollam superantibus; petalis rulyellis, seminibus margine membranaceo nullo v. tenuissimo. ( $\mathrm{Kgl.}$.)

\section{Sagina marltima bon (et Rehbeh.).}

Differt S. stricta Fries (et Engl, Bot. 2195) foliis longioribus, ciliatis, apice vix mucronatis, et calycibus fructiferis globosis, (Rgl.).

\section{Alsine tonulfolla Crntz. $\delta$, hrachypetala Dedeb.}

Glabra, petalis calyce $1 / 3-1 / 2$ brevioribus; sepalis plerumque obscure trinerviis et margine late scariosis.

Caules forma vulgari bumiliores.

Semina misit Schrenk ex Alatan. (Rgl.).

\section{Hermannia discolor otto et Dietr.}

Ramis pilis ramosis et glanduliferis subvillosis; foliis longe petiolatis e basi subcordata ovatis, obtusis, duplicato-crenatis, ṕlicatis, supra viridibus pilis stellatis hirtis, "sublus pilis stellatis albido tonientosis; stipulis parvis linearibus; racemis terminalibus; floribus nutantibus, plerumque subfasciculatis; pedunculis pedicellis calycibusque glandulosopilosis; calycibus campanulatís, acute dentatis.

Frutex $2-3$ pedalis, ramis laxis recurvatis. Flores magni, aurei. Pedicelli basi bracteis linearibus suffulti. Racemorum glomeruli distantes, 2-4 flori. - Huc pertinet verosimiliter H. aurea Otto et Dietr. Ally. Gartenz. VIII. 321. (Kgl.).

\section{Bunchonla emargluata Ifgl.}

Glabra; ramis tuberculatis; foliis oblongis, obtusis, emarginatis, subundulatis, integerrimis; racemis folio duplo brevioribus; stylo unico; stigmate vix bilobo.

Frutex $2-3$ pedalis, cortice brunneo-tuberculato. Folia opposita, sempervirentia, nitida, breviter petiolata, basi rotundata vel attenuata et subtus 2-4 glandulosa, juhiora supra pilis raris adpressis adspersa, deinde glaberrima, usque 4 pollices longa et $1 / 2$ poll. lata. Racemi axil- 
lares, simplices, pauciflori. Pedunculus strigilosus pilosulus. Flores flavi. Caly $x$ basi 8-10 glandulosus. Petala unguiculata, lamina subrotunda, margine crenulato-crispa.

Semina cl. Chappy e Cuba misit. (Rgl.).

Dodonaea angustissima Hort. DC. prodr. 1. 617.

D. Cunninghami (Hort. Petrop.).

Foliis linearibus latitudine decuplo longioribus, punctis albis superne stigmatosis.

Frutex ramosissimus, omnino glaber, usque 10 ped. altus, ramis tenuibus. Folia alterna vel opposita, anguste linearia, canaliculata, fere pollicem longa, vix lineam lata, juniora glandulosa, seniora praecipue superne stigmatosa; corymbis terminalibus, paucilloris; capsulis tetrapteris, subrhombeis, rotundatis. - D. acerosa Lindl. differt corymbis axilla bus. (Rgl.).

Dodonaea IIIta Ferd, Miilter (Herb.).

Dodonaea sp. nov. Behr in Linnaea 20, 634, no. 148.

Ramulis (subtrigono-) angulatis; foliis oblongo-cuneatis, obtusis vel acutis vel emarginatis vel superne tridentatis, glabris, utrinque glandulosis, junioribus verniceo-viscosis, adultioribus albo-glandulosis (in Mulleri specim. exsicc. foliis omnibus verniceo-glandulosis); floribus dioicis, in panicula racemosa terminali dispositis; sepalis ovatis, pilosis. ( $\mathrm{Kgl}$.).

\section{Polygala Sprengellana EekI. et zeyh.}

(Herb. Eckl. no. 147).

Virgato-ramosa; ramulis plus minus aggregatis, teretibus, junioribus folisque pubescentibus deinde glabris ; foliis alternis, cuneato-oblongis, ex apice obtuso mucronulatis vel subretusis, obscure viriabibus; racemis terminalibus; floribus petiolatis, subpendulis; petiolis flore brevioribus; brai teis $\mathbf{3}$ ad basin pedicellorum subovalibus, caducis, parvis, ciliolatis; carina ampla cristata.

Folia breviter petiolata, $3 / 4$ poll. longa, $1 / 6$ poll. lata. Flores dimidio mibores quam in Polygala speciosa, cui affinis est. P. simplex Burch. caule simplice cum foliis glabro differt. (Rgl.).

\section{Celastrus aquifolius hort. Petrop.}

Inermis, erectus, glaber; ramulis junioribus angulatis vel compressis (nee quadrangulis); foliis alternis oblongis, spinoso-siuuato-dentatis; peduneulis axillaribus, confertis.

Folia coriacea, $2^{1} / 4$ poll. longa, $3 / 4$ poll. lata. Celastro quadrangulo Schrad. affinis.

Patria ignota. (Rgl.).

\section{Maytenus Eledellanus Hort. E'errop.}

Ramulis tereliuscuslis; foliis ovatis vel oblongo-obovatis, obtusis, petiolatis; repando-crenulatis; paniculis axillaribus, foliis 2 -4-plo brevioribus; calycis dentibus obtusissimis, petalis ovatis, acutis.

Affinis Mayteno oblusifulio MarL, an varietas?

C. Riedel semina misit e Brasilia. (Rgi.). 


\section{Collecla crenata bejo.}

Fruticosa, glabra, spiuosa; foliis oppositis, membranaceis, breviter petiolatis, lanceolatis vel ovato-lanceolatis, apice oblusis et vix mucronulatis vel acutiusi ulis, repando crenulatis, crenis glandula parva terminatis, supra nitidis subtus pallidioribus, usque 2 poll. longis et $3 / 4$ poll. latis; floribus axillaribus, aggregatis; pedicellis calyce longioribus, filiforaibus, vix 1/4-pollicaribus; calycibus urceolato-campamulatis, albis, 4-b-Jobis, lobis lanceolatis recurvis; staminibus lubo calycis adnatis et inter lobos ejusdem insertis, exsertis; disco hypogy no, crasso, lobulato.

Patria iguota. (Kgl.).

\section{Euplogrbia rubricaulls Itgl.}

Caule fruticoso, erecto, ramoso; ramis sparsis ; foliis alternis, oblongolanceolatis, apice rotundato-obtusis et vix mucrouulatis, integerrimis, basi attenuatis; umbella terminali, simplici, $3-3$ radiatir, foliis involu-. crantihus breviore; foliis floritibus oppositis vel ternis, rhombeo-ovatis, ex apice ohtuso mucrouulatis; flori s singulis vel 3 -4-nis; squamis latioribus, quim longis, utrinque rotundatis vel apice leviter emarginatis, pallidis.

Patria ignota. (R grl.).

\section{Agathosina acuminata villd.}

Foliis alternis, ovatis, subcordatis, acuminatis, patentibus, pubescentibus, ciliatis, punctatis; llofibus terminalibus capitatis; pédunculis calycibusque sulvillosis; bacca acuminata. Wendl. Coll. 79, 1.28.

3. Candollei Kgl.; folis longe acuminatis, pedunculis villosis; calycibus glabris, glandulosis. - Diosma acuminata DC. proitr. 1. 713.

$\gamma$. subcordata Rgl.; foliis oblusiusculis; pedunculis calycibusque ut genuinae. - Diosma cordata Hort. D. subcordata Hoffm. (Kgl.).

A

Foliis imbricatis (trigonis) obtusis, canaliculatis, glabriusculis, pedunculis villosis.

a. panciplora Kgl,; umbellis subtrifloris, pedunculis folio llorali vix longioribus.

ß. Lrevifolia Rgl.; umbellis 10-15 noris; pedunculis folia floralia superantibus.

Diosma brevifolia Lam. D. thyoides W.

Agathosma serpyllacea lickl. et Zeyh. no. 909.

$\gamma$. rosea; umbellis plurjlloris; pedicellis glabris, glandulosis, quam folia brevioribus; folis deinde palulis vel subrecurvis.

Agathosma th, oides Hort. Berol.

Glabra, verticillata, ramosa. Rami laxi, superne vix puberuli. Folia lanceolato-oblonga, calloso-ubtusa, crassiuscula, sparsa, deinde patula vel subrecurva, supra subcaualiçulata, subtus convexa nervo vix promithente, utrinque bifariam glandulosa. Flores in umbellam plurifloram termiualem disposili, louge pedicellati pedicelloque pulchre rosei. Pedicelli graciles glandulosi, ${ }_{2}$ pullicem longi. Sepala lauceolato-oblonga, obtusa, margine membranacea, glanduloso-punctata. Petala longe unguiculata, apice in lamiuam subrotundam explanata, stamina sterilia pelatis breviora, cuneato-oblonga, subtusinfra fupicen glandula minuta instricta, basi pubesentia. Stamina fertilia petalis longiora. An species propria? ( $\mathbf{K}_{b} \mathbf{l}$.). 
Dlosma rubra L. $\beta$. ehloroealyx wigl.

Calyce viridi, sepalis apice tantum rubescentibus.

Systemon Rgl. Gen. nov, Rutacearum.

Calyx inferne tubo brevi, limbo inaequaliter 5-lobo. Petala 5, hypogyna, linearia, inferne in tubum pentagonum conglutinata, apicibus liberis patulis. Stamina 5, quorum 4 fertilia, hypogyna, inclusa; filamenta brevia complanata et in tubum coalita ; antherae lineares, introrsae, biloculares, basi biloba affixae, liberae. Nectarium cupuliforme, germen cingens. Styli 5, basi distincti, apice in unicum coaliti; stigma capitatum, quinquecostatum.

Frutex caule simplici apice foliis magnis coronato. Flores extraaxillares corymboso-congesti. Galipeae affinis, differt autem filamentis in tubum coalitis.

\section{Bystemen Fischeri Rgl.}

Caule fruticoso simplici; foliis simplicibus, maximis, cuneato-oblongis, glabris, apicem versus attenuatis, obtusiusculis; florum cymis aggregatis densis, extra-axillaribus; calycis dentibus lanceolato-subulatis, elongatis, inaequalibus, tubo longioribus; staminibus 5 , fertilibus 4 ; filamentis in tubum coalitis.

Frutex excelsus, caule plerumque simplici et apice foliis coronato. Folia maxima, usque $1 \frac{1}{2}$ ped. longa et $3-5$ poll. latii; breviter pedicellata, cuneato-oblonga, apicem versus attenuata, obtusa, coriacea, integerrima, glaberrima, penninervia, nervis arcuatis marginem versus anastomosantibus, minute pellucido-punctata, supra atroviridia nitentia, subtus pallidiora. Flores extraaxillares, in cymas aggregatas densas congesti; singuli breviter pedicellati. Calyx puberulus, tubo brevi, limbo inaequaliter $\$$-lobo, lobis lani eolato-subulatis, apice patulis, tubo brevioribus. Petala extus puberula, albida, a basi ad medium in tubum subpentagonum coalita, apice patentia et lobos lineari-lingulatos obtusiusculos 5 simulantia. Stamina 5 , glabra, corollae basi inserta; filamentis brevibus in tubum coalitis; antheris linearibus liberis inclusis. Nectarium cupuliforme, germen cingens. Styli 5, basi distincti, apice in unum coaliti; stigmate quinque-costato. (Rgl.).

\section{Oxalls mlerantha Hert.}

Caule abbreviato; petiolis pedunculis calycibusque pilis patentibus hirsutis; foliis vel simplicibus et $2-3$-lobis, vel $2-3$ foliatis; foliolis late obcordato-oblongis, supra glabris, subtus pilis simplicibus adspersis: stipulis nullis; pedunculis $\mathbf{1}-\mathbf{4}$ floris, folio brevioribus vel longioribus; pedicellis recurvatis; floribus parvis, luteis; sepalis lineari-lanceolatis; petalis brevioribus; stylis stamina aequantibus.

Radix annua. Affinis Oxali strictae $L$.

Patria ignota. (Rgl.).

\section{Beutzla erenata sleb, et Zuce. $\beta$. angustifolla $\mathrm{kgl}$}

Foliis ovato-lanceolatis, utrinque attenuatis, crenulatis apiculo crenulae incurvo, breviter petiolatis, ramulis pedunculisque utrinque pilis stellatis scabriusculis; racemis terminalibus; floribus nutantibus; calycibus pilis stellatis canis, laciniis ovatis acutis; staminibus tridentatis, antheris pilis stellatis hirtis. 
Differt praecipue foliorum formạ sicut racemis simplicibus ab icone atque foliorum margine a descriptione Sieboldtiana. (Rgl.).

\section{Epllobjum palustre F..}

a. linearifolium Rgl.; adpresse pubescens; foliis lineari-oblongis, vix denticulatis.

B. lineare Hort.

$\beta$. virescens; caule adpresse pubescente; foliis lanceolatis vel linearilanceolatis, vix denticulatis vel integerrimis; supra glabris, nitidis, subtus in nervis tantum vix pildsis.

$\gamma$. Schmidtianum; adpreșse puberulum; foliis oblongo-lanceolatis, denticulatis.

E. Schmidtianum Rostk. (Rgl.).

Callntemon lanceolatum BC. $\beta$. sparmum IBgl.

Metrosideros citrina Curt. Bot. Mag. tab. 260.

Differt floribus sparsis axillaribus.

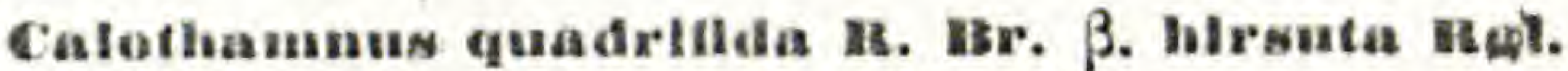

Foliis omnihus hirsutis, calycis tubo pubescente. (Rgl.).

\section{REubus hoblils Hort. Angl.}

Caule erecto, subangulato, aculeis minimis adsperso, cano-piloso; foliis ternatis trilobisve supra pilosis, subtus cano-puberulis; foliolis ovatorhomboideis, acuminatis vel lateralibus basi postice cordatis integris vel bilobis, omnibus duplicato-dentatis; pedunculis axillaribus terminalibusque bi-plurifloris; floribus pulchre roseis; sepalis canis; vvatis, longe aristatis, quam petala elliptica, duplo brevioribus.

Affinis Rubo spectabili Pursh. Accepimus ex hortis anglicis.

Patria ignota. (Rgl.).

\section{Pultenaea Lindleyana Ingl.}

Pultenaea subumbellata Lindl. Bot. Reg. 1632 nec Hook.

Ramis cinereo-pilosis; foliis sparsis, breviter petiolatis, linearibus, obtusiusculis, glabris vel pilosulis, superioribus erecto-patentibus; capitulis terminalibus, $4-8$-floris; floribus erecto-patentibus; calycibus sericeo-pilosis, bilabiatis, labio superiore obtusiuscule bidentato, inferiore trilobato, lobis lineari-lanceolatis, acutis, tubum aequantıbuss.

Planta nostra cum icone tab. 1632 Bot. Reg. congruit. Folia atroviridia, $1 / 3$ poll. longa. Flores aurei rubro variegati, vix $1 / 2$ poll. longi. P. subumbellata Hook. differt: ramis junioribus pilosulis; folis oblongolinearibus, glabris, superioribus horizontaliter vel reflexo-patentibus; capitulis terminalibus, 12-plurifloris; floribus horizontaliter patentibus; calycibus hirsutis in icone Bot. Mag. t. 3254 vix apice hirtis), hilabiatis; labio superiore bidentato, inferiore tridentato, dentibus óbtusis, tubo brevioribus; corollis majoribus. ( $\mathrm{H}$ gl.).

\section{Davlesia mimesolden Dryand. $\beta$. soligna met.}

Foliis rectioribus angustioribus; floribus aureis, intus vix maculatis. (Rgl.). 
Doryeniun eaneasleum M. IB. $\beta$. dentatum Egl.

Foliis sinuato-dentatis, dentibus mucronatis, ligulis angustioribus dignoscilur a forma genuina. (Kgl.).

\section{Grvum ehllense $\mathrm{F}$, et $\mathrm{M}$.}

Anuuum puherulum; caule humili, tetragono, e basi ramoso: foliis $2-3$ jugis, foliolis ellipticis mucronulatis, cirrho simplici vel furcato: stipulis semisagittatis, lanceolatis, integerrimis; pedunculis folio paullo brevioribus, $2-3$-floris; calycis laciniis setaceis lubum aequantibus; leguminibus lineazibus, tetraspermis compressis, (Hgl.).

(Semina misit Cuming e Chili).

Viela grandifiora seop. Var. villokn Egl.'

V. Biebersteinii Meyer in Hort. Petrop.

Sericeo-pilosa; foliis $6-8$ jugis; foliolis oblongo-linearibus, mucronatis; stipulis inferioribus semisagittatis, superioribus lanceolalis; leg. Linibus villosis. ( $\mathrm{Kgl}$.).

Tieia lutea $1 ., \gamma$. glabra.

Foliolis ovato-oblongis, apice obtusis vel truncatis mucronatisve, leguminibus glabris.

Semina misit Buhse e Persia. (Rgl.).

Dr. Liber Baro a Kuemter,

Collega.

Petropoli Decembri 1856.
R. reger.

F. Hisuieke,

1.. Uneh.

\section{ПЕЧАТАТЬ ПОЗВОАЯЕТСЯ,}

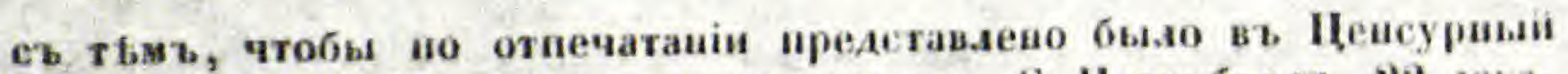

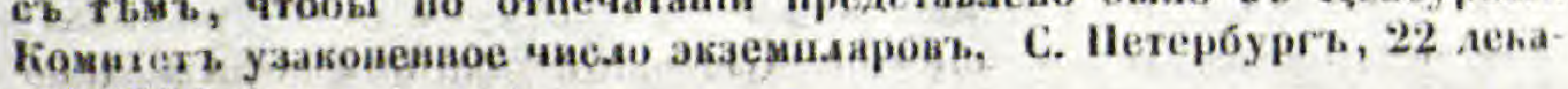
бра $18 \$ 6$ rosa.

Цеисорь А. Фрейлангв. 


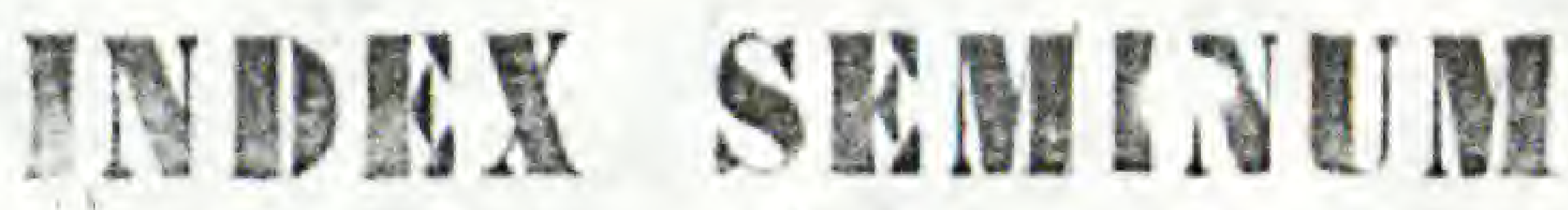

QUAE

\section{HORTUS BOTANICUS IMPERIALIS PETROPOLITANUS}

\author{
PRO \\ MUTUA COMMUTATIONE OFFERT.
}

Accedunt animadversiones botanicae nonnullae:

a. = planta annua. b. = planta biennis $p=$ planta perennis. $1=$ planta lignosa. $\mathrm{fr}=$ frigidarium. c. - caldarium. Plantae non signatae sub dio crescunt.

\section{7.}

Abutilon Avicennae Gärt. a. c.

- Behrianum F. Miill. a. c.

- indicum Don. a. c.

Acacia armata $R$. Br. 1. fr.

- Julibrissin Willd. 1. fr.

Acaena trifida var.glabrescens $R g l$., hoke. p. fr.

Acalypha brachystachya Horn.a.c.

- caroliniana Walt. a. c.

Acanthocephalus amplexifolius

har., hir. a.

Acer tataricum $L$. 1 .

Achillea alpina $\boldsymbol{L}$. p.

- leptophylla MB. p.

Achyranthes fruticosa Lam. 1. c.

Achyrophorus hispidus $D C$. p.

- maculatus Scop. p.

Acinos graveolens $\boldsymbol{L} \boldsymbol{k}$. a.

Acnida rhyssocarpa Michx, a. c.

- tuberculata Moq. a. c.

A conitum Anthora L. p.

- barbatum Patr. $\beta$. hispidum Ledeb. p.

- Kusnetzowii Rchb. p.

- Lycoctonum $\boldsymbol{L}$. $\beta$. fl. ochrol. a. calcari spirali Ledeb.p.

- Napellus L. var. (A.) Bern. hardianum Hehl. p.
Aconitum Napellus L. var. compactum rubellum Rchb. p.

- var. (A.) Eunkianum Rcht. P.

- var. (A.) laetum Rehb. P.

- var. (A.) pyramidale Mill. p.

- orientale Mill. p.

- variegatum $I$. p.

- - var. (A.) nasutum Fisch. p.

Acroclinium roseum Hook. a.

Adenophora denticulata Fisch. var.

latifolia p.

- marsupiftora Fisch. p.

Adesmia muricata $\boldsymbol{D C}$. a.

Adhatoda furcata Nees. I. c.

Adonis caudata Stev. a.

- squarrosa Stev, a.

- vernalis $\boldsymbol{L}$, p.

Aegilops speltoides Tausch, a.

Aerva lanata Juss. a. c.

Aethionema Buxbaumii DC. a.

- heterocarpum Gay. a.।

Agrimonia calfra E. Mey. p. fr.

- leucantha. Kze. p. 
Agrimonia pilosa I.edeb. p.

- procera Wallr. p.

- repens $\boldsymbol{L}$. p.

Agrostis distans Kze. a.

- lachnantha Nees. a.

- pallida DC, a.

A ira capillaris Host. a.

- caryophyllea $\boldsymbol{L}$. a.

- Lagascae Kth. a.

Airopsis pulchella Ten. a.

Alchemilla alpina $\boldsymbol{L}$. p.

- arvensis Scop. a.

- pubescens $\boldsymbol{M B}$. p.

- vulgaris $\boldsymbol{L}$. $\beta$. subsericea Koch. $\mathrm{p}$.

Alfredia cernua Cass. p.

Allitim ingulosum $\boldsymbol{L}$. p.

- cardiostemon Fisch., Mey. p.

- carinatum $\boldsymbol{L}$. p.

- coeruleum Pall. p.

- - $\quad-\gamma$. album H. Petrop. p.

- flavidum Ledeb. p.

- hynenorhizum Ledeb. p.

- Moly L. p.

- obliquum L. p.

- odornm L. p.

- polyphyllum Kar., Kir. p.

- rotundum $\boldsymbol{L}$. $\beta$. Waldsteinianum $R$., $S$. p.

- senescrns $\boldsymbol{L}$. p.

- strictun Schrad. p.

- tataricum L. fil. p.

Alnus viridis $D C$. 1 .

Alonsoa incisifolia $\boldsymbol{R} ., \boldsymbol{P}$. a.

- Warszewiczii Rgl. b. fr.

Alopecurus geniculatus $\boldsymbol{L}$. p.

- utriculatus Pers. a.

Alsine giobulosa C. A. Mey. a.

- laricifolia Viahlbg. p.

- verna Bartlg. $\beta$. alpina

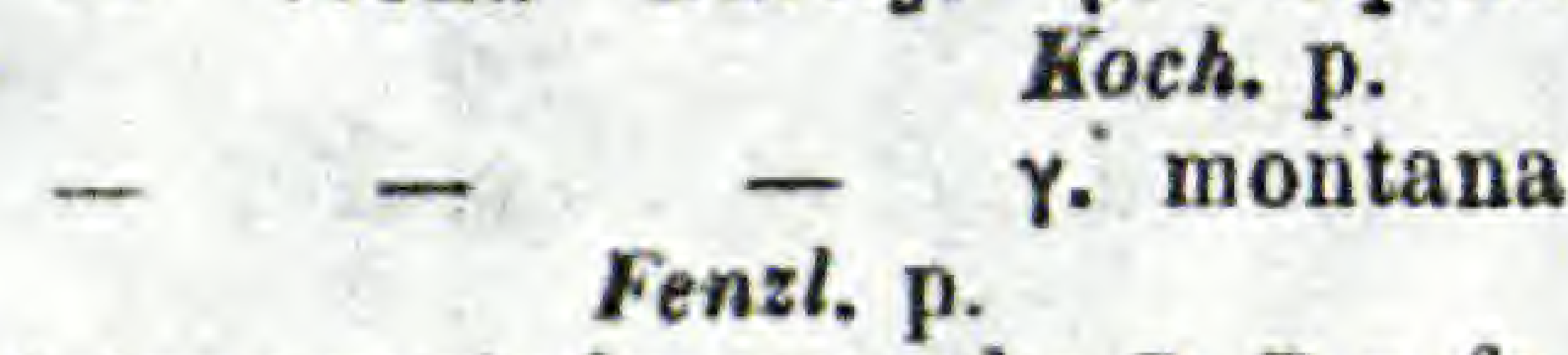

Alstroemeria haemantha R.,P.p.fr. Alternanthera Achyrantha $\boldsymbol{R}$. Br. a. c.

- coidea R., S. a. c.

- sessilis $\boldsymbol{R} . \boldsymbol{B r}$. a. c.

- pinosa R., S. a. c.

Althaea ficifolia Cav. p.

Alyssum argenteum Vilm. p.
Alyssum hirsutum $\boldsymbol{M B}$, a.

- maritimum Lam. a.

- micranthum Fisch., Mey. a.

- minimum Schlchtd. vir. a.

- rostratum Stev. b.

- Szovitsianum Fisch., Mey. a.

- umbellatum Desv. a.

Amberboa Lippii $\boldsymbol{D C}$. a.

Amblogyna polygonoides Rafin. a.

Amblyolepis setigera $\boldsymbol{D C}$. a. c.

Ambrosia artemisia efolia $\boldsymbol{L}$. a. c.

- maritima $\boldsymbol{L}$. a. c.

Amelanchier Botryapium DC. 1.

Amellus tridactylus $D C$. a.

Ammannia aegyptiaca Willd. a. c.

- auriculata Willd. a. c.

- diffusa Willd. a. c.

- latifolia L. a. c.

- sanguinolenta Sw. a. c.

- senegalensis Lam. a. c.

Ammobium alatum $R$. Br. b. fr.

Amsinckia angustifolia Lehm. a.

- intermedia Fisch., Mey. a.

- lycopsioides Lehm. a.

- spectabilis Fisch., Mey. a.

Amygdalus nana $\boldsymbol{L}$. $\mathrm{I}$.

Anacyclus purpurascens Pers. a.

Anarrhinumbellidifolium Desf. b.fr.

- $\quad$ - fl. albo b. fr.

- Duriminium Chav. b. fr.

Anastatica hierochuntica $\boldsymbol{L}$. a. c.

Anchusa hybrida Ten. a.

- Milleri Willd. a.

- stylosa MB. a.

Andersonia sprengelioides $\boldsymbol{R}$. $\mathbf{B r}$.

$$
\text { 1. fr. }
$$

Andromeda buxifolia $L$. I. fr.

Androsace commutata Schlchtd. p.

- elongata $\boldsymbol{L}$. a.

- Gmelini Gaerın. b.

- lactea L. p.

Androsaemum ofticinale All. p.

Andryala laxiflora $D C$. a.

Anemone dichotoma L. p. (Amur.)

- multifida Poir. $\gamma$. Hudsoniana $p$.

- narcissiflora $L$. p.

- silvestris $\boldsymbol{L}$. p.

- virginiana $\boldsymbol{L}$. p.

Angianthus filifolius C. A. Mey. a. Anisolotus Wrangelian. Bernh. a. Anoda cristata Schlchtd, a. 
Anoda hastata Cav. a.

Antennaria alpina Gaertn. p.

Anthemis Chia $\boldsymbol{L}$. a.

- chrysantha Gay. a.

- granatensis Boiss. a.

- maritima L. p. fr.

- Neilreichii Ortm. a.

Anthoxantum amarum Brot. a.

- aristatum Boiss. a.

- gracile Biv. a.

- ovatum Lag. a.

Anthriscus Cicutaria Duby. p."

- laevigata Griseb. p.

- nemorosa Spreng. p.

- torquata Duby. p.

Anticlea glauca $\boldsymbol{K} t h$. p. fr.

Antirrhinum Orontium L. a.

Anychia capillacea $D C$. a.

- dichotoma Nichx. a.

A phanostephus ramosissimus DC. b. p. fr.

Aquilegia fragrans Benth. p.

- hybrida. Sims. p.

- jucunda Fisch., Lall. p.'

- sibirica Lam. p.

$\div \quad$ - - var.irc tiana

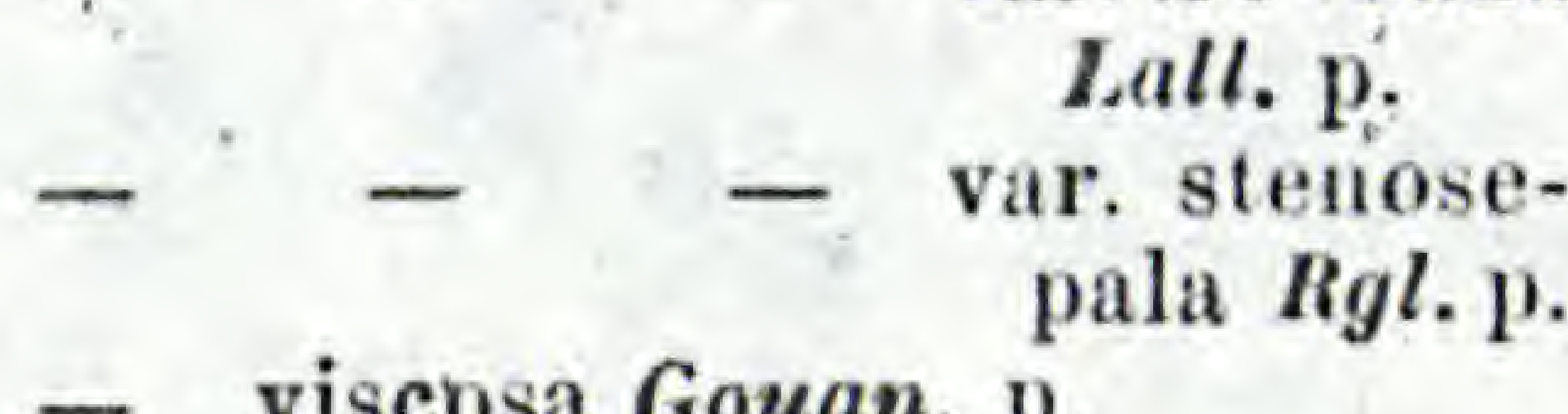

- viscosa Gouan. p.

Arabis arenosa Scop. a.

$$
\text { - } \beta \text {. lutea. } p \text {. }
$$

- - var. albiflora. a.

- bellidifolia Jacq. p.

- ciliata R. Br. p.

- $\quad$ - B. hirsuta Koch. p.

- collina Ten. p.

- declinata Schrad. p.

- heteromalla Schrad. $\alpha$. eriocarpa p.

- muralis Bertol. p.

- pendula $L$. p.

- petraea Crantz. p.

- pseudoturritis B rișs, Heldr. b. fr.

- stenopetala Willd. p.

- stricta Huds. p.

Arbutus Andrachne L. 1 . fr.

- Uneslo L. I. fr.

- - fr. albo 1. fr.

Arehangelica decurrens Ledeb. p. Arctostaphylos uva ursi Spr. 1.
Arenaria ciliata $\boldsymbol{L}$. p.

Arenaria conimbricensis Brot. a.

- glomerata .MB. a.

- graminea C. A. Mey.

- longifolia MB.p.

- muralis Sieb. a.

Aristolochia ciliata Hook. 1. c.

Armeria alpina Will $l$. p.

- elongata Ho/fm. p.

- plantaginea Willd. p. fr.

- pungens $R$. S. p. fr.

Arnica Chamissonis Less. p.

Artemisia St:lleriana Bess. p.

Asclepias nivea $L$. l. c.

Asperula setosa Jaub., Spack. a.

Aster abbreviatus Ners. p.

- adulterinus Willd. p.

- alpinus L. p.

- bellidiflorus Willd. $\beta$. angustus DC. p.

- brumalis Nees. p.

- cyaneus Hoffm. 1 .

- divergens Ail. p.

- firmus Nees. p.

- fragilis Willd. p.

- $\quad$ DC. p. $\beta$. subinteger
- laevis L. p.
- lanceolatus Willd. p.
- laxus Willd. p.
- leucanthemus Desf. p.
- longifolius Lam. $\gamma$. eminens

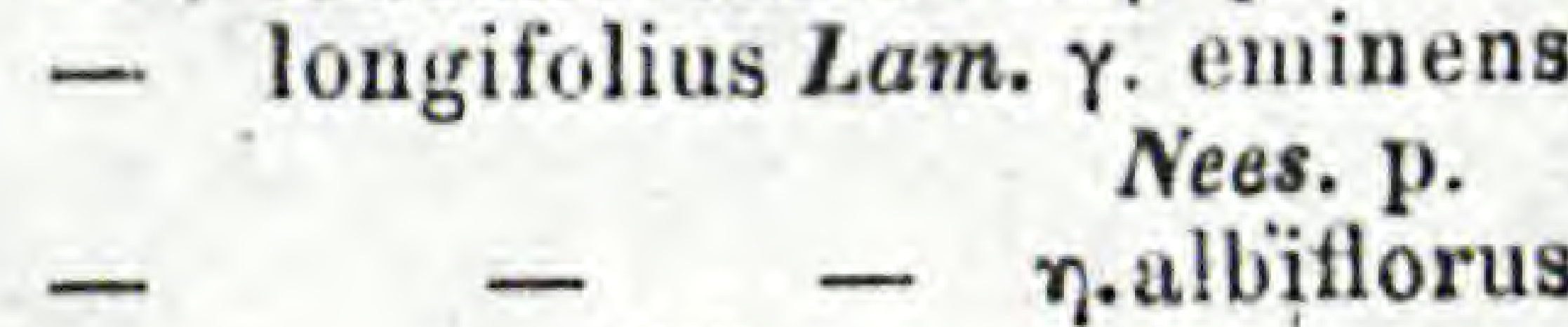
DC. p.

mutabilis Ait. p.

Novi-Belgii Nees. $\beta$. squarrosus Nees.p.

- $\quad-\delta$. floribun. dus DC. p.

peregrinus $\boldsymbol{P}$ ursh. p.

Radula Ait. p.

sibiricus $\boldsymbol{L}$.

squarrosulus Nees. p.

- Nees. var. jngustifolius $p$.

- $\quad$ var. pallidiflorus $\mathrm{p}$.

tardiflorus Nees. p.

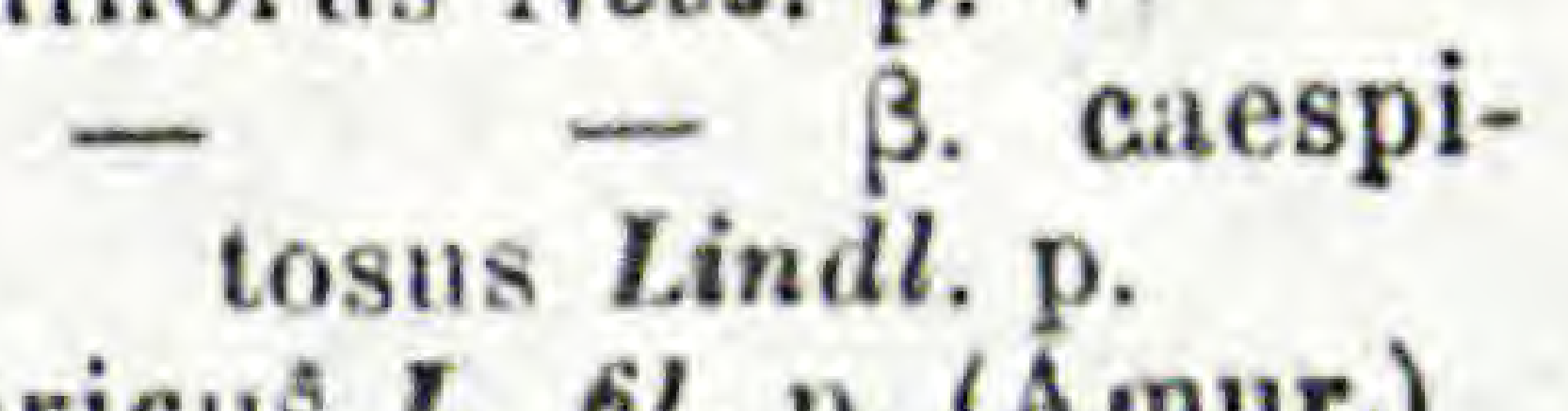

tataricus L. fll. p. (Âmur.) 
Asterolinum stellatum $\boldsymbol{L} \boldsymbol{k}$. a. Astrantia Biebersteinii Trautv. p. - helleborifolia Salisb. p. Aubrietia deltoidea $\boldsymbol{D C}$. $\mathrm{p}$. Augustia caffira $K l$. l. c.

- Dregei $\boldsymbol{K l}$. 1. c.

Bae-ia chrysostoma Fisch., Mey. a. Bapusia australis $\boldsymbol{R}, \boldsymbol{B r}$. p. Barbarea arcuata Rchb. p.

Baltimora recta $L$. a. c. Barkhausia alpina $\boldsymbol{D C}$. a. Bartonia aurea Lindl, a. c. Begonia semperflorens Lk., Otto. 1. c.

Bellevalia comosa $\boldsymbol{K} t \boldsymbol{h}$. p.

$$
\text { - }- \text { var. prae- }
$$$$
\operatorname{cox} \text { H. Petrop. p. }
$$

- romana Rchb. p.

Bellis annua $\boldsymbol{L}$. a.

- integrifolia. Michx, a.

Bellium bellidioides $\boldsymbol{L}$. p. fr.

Betckea samolifolia $\boldsymbol{D C}$. a.

Betonica grandiflora Steph. p.

Betula fruticosa Pall. I.

- Gmelini Bye. 1.

Bidens ferulaefolia $\boldsymbol{D C}$. a. c.

Biotia discolor Maxim. p. (Amur.)

- latifolia DC. p.

- macrophylla DC. p.

Blitum capitatum $\boldsymbol{L}$. a.

- glaucum Koch. a.

- polymorphum C. A. M. a.

- virgatum $\boldsymbol{L}$. a.

Biscutella apula $\boldsymbol{L}$. a.

- auriculata $\boldsymbol{L}$. a.

- erigerifolia $\boldsymbol{D C}$. a.

- lyrata L. $\alpha$. hispida Rgl. a.

- - - $\beta$. glabra Rgl. a.

- - - - ciliata Rgl. a.

Blumenbachia insignis Schrad.a.c. Boronia alata $S m$. l. fr.

- fastigiata Bartl. 1. fr.

Bossiaea heterophylla $S m$. 1. fr.

- linophylla $R$. Br. l. fr.

- paucifolia Benth. 1. fr.

Botryadenia Gmelini Fisch., Mey.

$$
\text { p. fr. }
$$

Botryanthus odorus $\mathrm{K} t \boldsymbol{h}$. p. Szovitsianus C. A. Mey. p.

Bowlesia incana $\boldsymbol{R} ., \boldsymbol{P}$, a. c.

- tenera $S_{j} \boldsymbol{y}$. a. c.

Brachycome calocarpa $\boldsymbol{F}$. Mill. a.
Brachycome diversifolia Fisch., Mey. b. p. fr. iberidifolia Benth. a.

Brachycoris parviflora Schrad. a.c. Brassica oxyrrhina Coss. a.

- Schimperi Boiss. a.

Braya siliquosa Bge. p.

Bromus adoensis Hochst. a.

- arduenensis Kunth. a.

- brachystachys Hornung. a.

- commutatus Schrad. a.

- diandrus Curt. B. spiculis scabris rubentibus a.

- $\quad-\gamma$. spiculis pubescentibus. a.

- divaricatus Rhode. a.

- Ianceolatus Roth, a.

- madritensis $L$. a.

- maximus Desf. a.

- mollis $\boldsymbol{L}$. a.

- oxyodon Schrenk. a.

- patulus M., K. a.

- pendulinus Schrad. a.

- racemosus $\boldsymbol{L}$. spiculis glabris a.

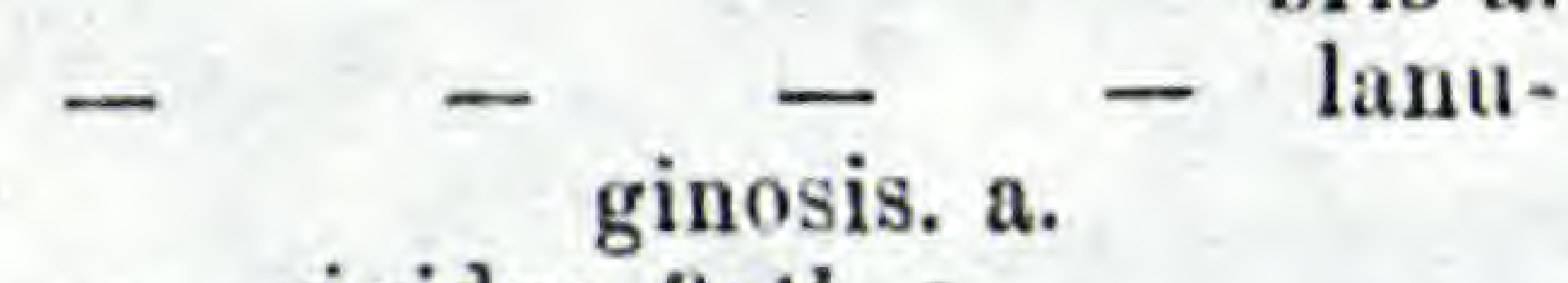

- rigidus Roth. a.

- rubens $\boldsymbol{L}$. a.

- Schraderi Kth. a.

- squarrosus $\boldsymbol{L}$. a.

- tectorum $\boldsymbol{L}$. a.

- vestitus Schrad. a.

- wolgensis Jacq. a.

Broteroa trinervata Pers. a.

Browallia elata $\boldsymbol{L}$. a. c.

- viscosa Humb., Bonpl. a. c.

Buffonia macrosperma Gay. a.

Bulbine annua. Willd. a.

- semibarbata Spr. a.

Bulliarda Vaillantii $D C$. a.

Bupleurum fruticosum l. fr.

- Gerardi Jacq. a.

Cajophora lateritia Benth. a. c.

Cakile maritima Soop. a.

Calais linearifolia DC. a.

Calmagrostis Epigeios Koth. $\gamma$. georgica Griseb. p.

- phragmitoides Hartm. p.

- stricta Spr. p.

- sylvatica $D C$. p.

Calamintha alpina Benth. p. 
Calamintha annua Sehrenk. a.

- Clinopodium Benth. p.

- Nepeta $\boldsymbol{L}$.., Hoffm. p.

- umbrosa Benth. p.

Calandrinia discolor Lindl. a. c.

Calceolaria glutinosa Heer., Rgl. $\beta$. californica $\mathrm{Kgl.}$ a.

\section{- pinnata $\boldsymbol{L}$. a.}

Calendula aegyptiaca Desf. a.

- arvensis L. a.

- brachyglossa Rupr. a.

- eriocarpa DC. a.

- gracilis DC. a.

- incana Willd. a.

- marginata Willd. a.

- microcephala Kralik. a.

- officinalis $\boldsymbol{L}$. a.

- palaestina Boiss. a.

- sicula Cyr. a.

- stellata Cav. a.

- - - var. cristata a.

- sublanata Rchb. a.

- suffruticosa Vahl. a.

- tripterocarpa Rupr. a.

Calimeris incisa $\boldsymbol{D C}$. p.

Callicarpa americana $L$. 1 . fr.

Callicephalus nitens $\boldsymbol{C}$. A. Mey. a.

Callichroa platyglossa Fisch., Mey. a.

Calliglossa Douglasii Hook., Arn. a Calliopsis Atkinsoniana Hook. b. ' - cardaminefolia DC. $\mathrm{k}$.

Callisace dahurica Fisch. p.

Callistachys lanceolata Vent. 1. fr.

- linearis Benth. l. fr.

- ovata Sims. 1. fr.

- retusa Lodd. l. fr.

Callistemon lanceolatum $\boldsymbol{D C}$. I. fr.

- lineare DC. l. fr.

- pinifolium DC. 1. fr.

- rigidum $\boldsymbol{R} . \mathrm{Br}$. l. fr.

- speciosum DC. l. fr.

Callitris rhomboidea $\boldsymbol{R}$. Br.l. fr. Calophanes oblongifolius D.Don. at. Calothamnus clavata Mackay. I. fr:

- quadrifida $\boldsymbol{R}$. Br. 1. fr.

- schoenophylla Schauer.l.fr. Calotis cuneifolia $\boldsymbol{R}$. Br. a. c.

Calyptrostigma Middendorffiana Trautv., Mey. 1.

Campanula americana $\boldsymbol{L}$. p.

- bononiensis $\boldsymbol{L}$. p.
Campanula carpatica $\boldsymbol{L}$. p.

- Erinus $\boldsymbol{L}$. a.

- Hohenackeri Fisch., Mey. p.

- homallanthina Ledeb. p.

- lamiifolia $M B$. p.

- Langsdorffiana Fisch. p.

- latifolia $\boldsymbol{L} . \boldsymbol{\gamma}$. eriocarpa $\boldsymbol{A}$. DC. $\mathrm{p}$.

- Coefflingii Brot. a.

- Loreyi Pollin. a.

- punctata Lam. p.

- retrorsa Lab. a.

- rotundifolia $L$. $\delta$. lancifolia Koch. p.

- sarmatica Ker. p.

- thyrsoidea $L$. p.

Canna limbata Rosc. $\beta$. unicolor hgl. p. c.

- maculata Lk. p. c.

- patens Rosc. p. c.

- Sellowii H. Berol. p. c.

Capnophyllum africanum Gärtn. a.

Capsella pauciflora Koch. a.

- procumbens Fries. a.

- - ' $\gamma$. integrifolia. Koch, a.

Caragana arborescens L.am. 1.

- microphylla DC. 1.

Cardamine macrophylla Willd. p.

Carduus pycnoceohalus $\boldsymbol{L}$. a.

- tenuiflorus Curt. a.

- thessalus Boiss., $\boldsymbol{H}_{q_{i}}$. b. fr.

Carex atrata $\boldsymbol{L}$. p.

- caucasica Stev. p.

- divulsa Good. p.

- Gmelini Hook. p.

- incurva Lightf. p

- leiorhyncha C. A. Mey. p.

- leporina $L . \beta$.argyroglochin Koch. p.

- microglochin Wahlbg. p.

- stipata Mriihlog. p.

- straminea Willd. p

Carmichaelia australis $\boldsymbol{R} . \boldsymbol{B r}$. 1. fr. Carrichtera Vellae DC. a.

Casuarina pumila Otto, Dietr. I. fr. Celosia adoensis Hochst., Steud. a. $c$.

- margaritacea $L$. a. c.

Celsia orientalis $\boldsymbol{L}$. a.

Cenia geminata Kze. a.

Cenocline globifera $C$. Koph, a. 
Cenolophium Fischeri Koch. p.

Centaurea adpressa Ledeb. p.

- depressa $\boldsymbol{M B}$. a.

- diluta Ait. a.

- involucrata Desf. a.

- leptacantha Fisch., Mey. a.

- macrocephala Muss. Puschk. p.

- maculosa Lam. b.

- phrygia L. p.

- pseudophrygia C. A. Mey.p.

- pullata $\boldsymbol{L}$. a.

- sordida Willd. $\beta$. purpurascens Koch. p.

- spinulosa Rochel. p.

Cephalophoraaromatica. Schrad.a. Cerastinm brachypetalum Desp. a.

- campanulatum Viv. a.

- dahuricum Fisch. p.

- dichotomum L. $\boldsymbol{\gamma}$.glandulosum a.

- glutinosum Fries. $\alpha$. obscu-

rum Koch. a.

- - $\quad$ - $\beta$. pallens Koch. a.

- illyricum Ard. a.

- latifolium L. $\alpha$. latifolium Ledeb. p.

- manticum $\boldsymbol{L}$. a.

- multiflorum C. A. Mey. a.

Cercis Siliquastrum $\boldsymbol{L}$. $\boldsymbol{f}$. carneis 1. fr.

Cerinthe aspera Both. a.

Guss. a.

ß. pallida

Chaenostoma foetidum Benth.a.

Chaerophyllum aureum $\boldsymbol{L}$. p.

- millefolium $\boldsymbol{D C}$. p.

Chamaerops humilis $\boldsymbol{L}$. I. fr.

Chamomilla nobilis Godr. p.

Chorispora tenella $\boldsymbol{D C}$. a.

Chrysanthemum grandiflorum

Willd. $\gamma$. subnudum $D C$. I.fr.

Cinna mexicana Beauv. p. fr.

Cirsium canum All. p.

- dealbatum $\boldsymbol{M B}, \mathrm{p}$.

- munitum MB. b.

- pendulum Fisch. p. (Amur.)

Cistus undulatus Dun. 1. fr.

Clarkia rhomboidea Dougl. a.

Claytonia gypsophiloides Fisch., Mey. a.
Cleistanthium nepalense $\boldsymbol{K z e}, \mathrm{p}, \mathrm{fr}$. Clematis angustifolia Jacq. p (Ainur.)

- integrifolia. L. p.

- lathyrifolia Bess. p.

- mandshuricaRupr.p.(Amur.)

Cleome pungens Willd a. c.

- violacea $\boldsymbol{L}$. a. c.

Clethra arborea Ait. 1. fr.

Clintonia pulchella Lindl. a.

Closia chilensis Rgl., Kcke. a.

Clypeola microcarpa Boiss. a.

Cnidium apioides $S p r$. p.

Cochlearia anglica $\boldsymbol{L}$. a.

- arctica Schlchtd. a.

- glastifolia Lin. b. fr.

- macrocarpa $W, \boldsymbol{K}$. p.

- officinalis $\boldsymbol{L}$. b.

Coelopleurum Gmelini Ledeb. p.

Colchicum umbrosum Stev. p.

Collinsia bartsiaefolia Benth. a.

- - fl. albo a.

- grandiflora Lindl. a.

- parviflora Lindl. a.

- sparsiflora Fisch., Mey. a.

Collomia heterophylla Hook. it.

- linearis Nutt. a.

- stenosiphon Kze. a.

Commelina communis $\boldsymbol{L}$. a. c.

Conioselinum Fischeri Wimm., Grab. p.

- univittatum Turcz. p.

Conoclinium janthinum Morr. 1. c.

Convolvulus undulatus Cav, á.

Corispermum elongatum Bge. a. (Amur.)

Cornucopiae cucullatum $\boldsymbol{L}$. a.

Cornus alba $L .1$.

Corydalis angustifolia $\boldsymbol{D C}$. p.

- glauca Pursh, a.

- speciosa Maxim. p.(Amur.)

Cosmanthus fimbriatus Nolte. a.

- viscidus $\boldsymbol{D C}$. a.

Cotoneaster acuminata Lindl. l. fr.

- frigida Wall. l. fr.

- laxiflora Jacq. fil. 1.

- microphylla Wall.1. fr.

- Nummularia Fisch., Mey.I.fr.

- rotundifolia Wall. I. fr.

- uniflora Bge. I.

- vulgaris Lindl. 1. 
Cotula anthemoides $L$. a.

- coronopifolia $L$. a.

- filifolia Thby. a.

- microcephala DC. a.

- pusilla Thbg. a.

- sororia DC. a.

Cousinia Hystrix C. A. Mey. p.

Crambe hispanica $\boldsymbol{L}$. a.

Crassula diaphana E. Mey.

Crataegus sanguinea Pall. I.

Cremocephalum cernuum Cass.a.c.

Crepis aurea Cass, crocea Froel.

- lodomeriensis Bess. b.

- sibirica $L$. p.

Crucianella angustifolia $\boldsymbol{L}$. a. - latifolia $\boldsymbol{L}$. a.

- patula $L$. a.

Crupina crupinastrum Vis. a.

Cryptantha microcarpa Fisch,, Mey, a.

Cryptomeria japonica Don. l: fr.

Cryptostemma calendulaceum $\boldsymbol{R}$. Br. $\beta$. lyratum $\boldsymbol{D C}$. a.

Cucumis cubensis Schrad. a. c. - dipsaceus Ehrenb. a. c.

Cucurbita aurantiaca. Willd. a. c.

- maxima Duchsne. $\beta$. Viridis Ser. a. c.

- microcarpa Lall. a. c.

- ovifera. L. a. c.

- verrucosa $\boldsymbol{L}$. a. c.

Cupressus fastigiata $D C$. 1 . fr.

- glauca Lam. 1. fr.

- horizontalis Mill. l. fr.

Cycnoseris australis Endl. a.

Cynoglossum canescens Willd. a.:

- glochidiatum Wall. a.

- lanceolatum Forsk. a.

- viridiflorum Pall. p.

Cyperus tenellus $\boldsymbol{L}$, a.' c.

- vegetus Willd. a. c.

Cytisus albus $L k$. 1. fr.

- chrysobotrys Fisch., Mey. 1. fr.

- ratisbonensis Schaeff. 1.

Dactylaena micrantha Schrad. a. c. Dactyloctenium aegyptiacum. Willd, a. c.

Daphne altaica Pall. 1.

Daucus abyssinicus $C . A . M e y$. b. fr. - glochidiatus Fisch., Mey. a.

- montevidensis $\boldsymbol{L} \boldsymbol{k}$. a.
Daticus muricatus $\boldsymbol{L}$. a. It

pulcherrimus Foch, b. fr.

Daviesia mimosoides Dryand. $\beta$. saligna Rgl. 1. fr.

Deckera canescens C. A. Mey. p.

Deeringia baceata Moq. l. c.

Delphinium azureum Michx, p.

läiopetalum." Rgl.p.

- cheilanthum Fisch. p.

- dictyocarpum DC. p.

- elatum L. $\alpha$. Ledeb p.
- $\quad$ - $\beta$. a. subglabrum Ledeb., p.
ß. b. hispidum Le- deb. p.
- $-\beta$. c. subciliatum Ledeb. p.

- grandiflorum $\boldsymbol{L}$. p.

- hybridum Willd. $\varepsilon$, dasyanthum Lall. p."

- lasiostachyum Fisch.,Mey.p.

- triste Fisch. p.

Desmodium canadense $\boldsymbol{D C}$. p.

Deutzia scabra Thbg. l. fr.

Dianella strumosa $\boldsymbol{K}_{t} \boldsymbol{r}$. p. fr.

Dianthus atrorubens All. p.

- capitatus $\boldsymbol{D C}$. p.

- cruentus Griseb. p.

- deltoides $\boldsymbol{L} . \boldsymbol{\beta}$. glaucus $\boldsymbol{K}$ och. p.

- fimbriatus $M B$. p.

- plumarius L. p.

- Pseudo-Armeria. MB. p. fr.

- Seguieri Vill. $\alpha$. Ledeb. p.

- - $\quad$ - L. Ledeb. p.

- superbus $\boldsymbol{L}$. p.

Dichrocephala latifolia $\boldsymbol{D C}$. a. - sonchifolia $D C$. a.

Dicliptera resupinata Juss. a. c.

Dictamnus albus $\boldsymbol{L}$. fl. striato. p.

Digitalis grandiflora Lam. p.

- nervosa Steud. p.

Dimetopia hirta Benth. a. c.

- isocarpa Bartl. a. c.

- Walpersii Bge. a c.

Diodia dasycephalaCham,,Schlehtd. p. c.

Diospyros Lotus $L$. I. fr.

Diplotaxis muralis DC. a.

- viminea DC. a.

- virgata DC.a. 
Dipteracanthus Schauerianus Nees.

I. c. dus Rgl. I. c.

Dircaea cardinalis Rgl. p. c.

Dischisma arenarium E. Mey. a. c. - capitatum Chois. a. c.

- clandestinum E. Mey. a. c.

Dodecatheon integrifolium Michx. p.

- Meadia. L. var. p.

Doronicum caucasicum MB. p.

Draba Aizoon. Whlbg. p.

- alpina $\boldsymbol{L}$. p.

- arabisans Michx. b.

- aurea Vahl. b.

- borealis DC. p.

- caroliniana Walt. a.

- contorta Ehrh. p.

- hirta L. p.

- incana $\boldsymbol{L}$. p.

- $\quad$ - a.legitima Lindbl. p.

- $\quad-\beta$ hebecarpa Koch. p.

- $\quad$ - o.axillaris Lindbl. p.

- $\quad$ - e. gracilis $\mathbf{R g l . p}$.

- Iohannis Host. p.

- lutea Gilib. a.

- minima Steud. a.

- rupestris $\boldsymbol{R}$. Br. p.

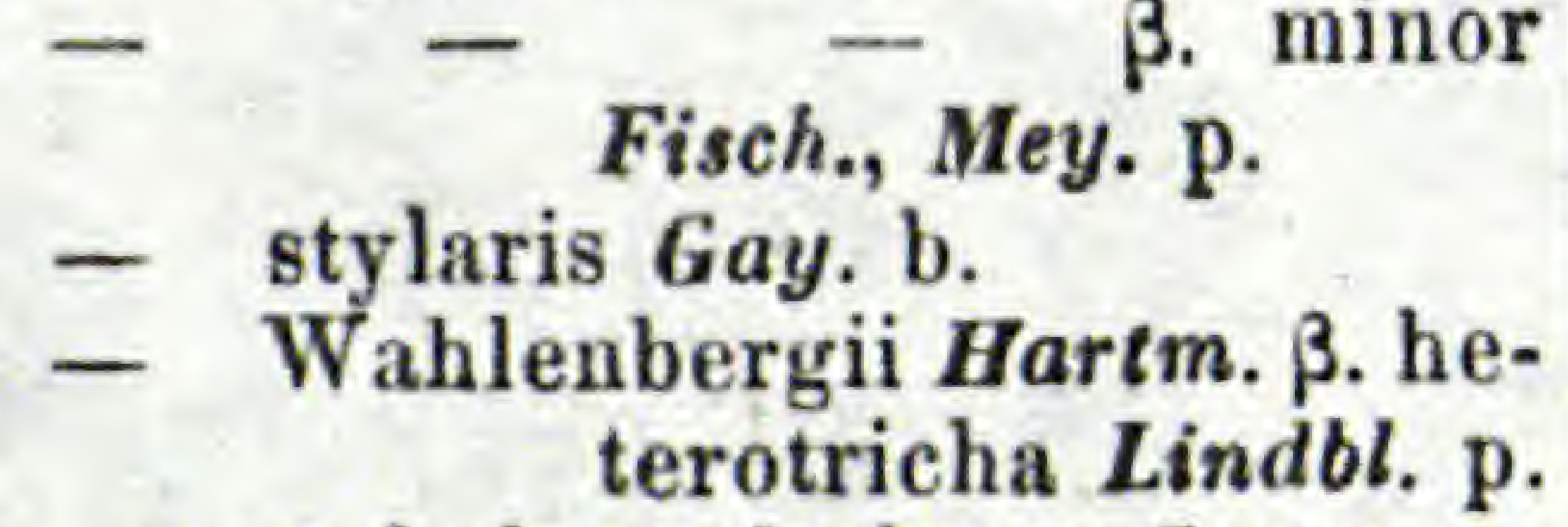

Dracocephalum altaiense $\mathbf{L}$ axm. p.

- nutans $\boldsymbol{L}$. p.

- parviflorum Nutt. p.

- peregrinum $\boldsymbol{L}$. p.

- Ruyschiana $L$. p.

- thymiflorum $\boldsymbol{L}$. a.

Dracopis amplexicaulis Cass. a. Drymaria villosa Cham., Schlchtd. a. c.

Dyckia remotiflora Otto, Dietr. l. c. Dyssodia alternifolia Rgl.,Kcke.a.c. Echenais Sieversii Fisch., Mey. b. Echeveria gibbiflora $\boldsymbol{D C}$. 1 . fr. Echinocactus corynodes $\boldsymbol{H}$. Berol. 1. c.
Echinops bannaticus Rochel. p.

- exaltatus Schrad. b.

Echinopsis Eyriesii P/ei/f., Otto. l. c.

- turbinata P/ei/f., Otto. 1. c. Echinospermum consanguineum Fisch., Mey. a.

- cristatum Bge. a.

- deflexum Lehm. b.

- marginatum Lehm. a.

- Redowskii Lehm. a.

- strictum Ledeb, a.

Echium creticum $\boldsymbol{L}$. a.

- plantagineum $\boldsymbol{L}$. a.

- pustulatum Sibth., Sm. b. fr.

- rubrum Jacq. b.

Eclipta erecta DC. a. c.

- hirsuta Bartl. a. c.

Elaeagnus argentea Pursh. 1.

Elaeocarpus cyaneus Sims. l. fr.

Eleusine coracana Pers. a. c.

- Figaraei Del. a. c.

- indica Gärtn. a. c.

Elymus caput Medusae $\boldsymbol{L}$, a.

- crinitus Schreb, a.

- geniculatus Curt. p.

- lativalvis $\boldsymbol{L} k$. p.

- mollis Trin. p.

- sibiricus $L$. p.

Engelmannia pinnatifida Torr., Gr. p. fr.

Ephedra andina Pöpp. 1. fr.

Epilobium affine Bong. a.

- alpinum $\boldsymbol{L}$. p.

- brevifolium Don. a.

- coloratum Mïhlbg. p.

- hypericifolium Tausch. p.

- mexicanum Moc., Sess. a.

- minutum Lindl. a.

- montanum $\boldsymbol{L}$. p.

- $\quad-$ Koch. $\delta$. collinum

- origanifolium Lam. p.

- palustre L. $\alpha$. linearifolium Rgl. p.

$$
\begin{gathered}
-\quad \beta . \text { virescens } \\
-\quad \begin{array}{c}
\text { Bgl. p. } \\
\text { - }
\end{array} \\
\text { num Kochmidtia- } \\
\text { Koch. p. }
\end{gathered}
$$

- parviflorum Schreb. p.

- roseum Schreb. p.

- tetragonum L. p. 
Epilobium uralense Rupr. a. virgatum Fries. p.

Erechtites valerianaefolia $\boldsymbol{D C}$. a. c. Erica baccans $L$. 1 . fr.

- lateralis Willd. 1. fr.

Erigeron acris $\boldsymbol{L}$. $\beta$. asteroides DC. b.

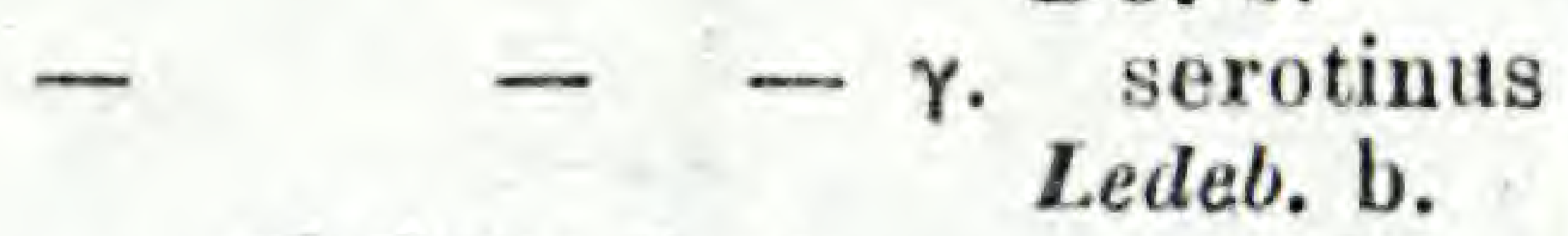

- alpinus $L . p$.

- $\quad-\quad-$ - . elatus Hook. p.

- armerifolius Turcz. $\alpha$. humilis Ledeb. b.

- asperulus Fisch., Mey. a.

- divaricatus Michx, a.

- Droebachiensis Miull. b.

- glabellus Nutt. p.

- glabratus Hoppe. p.

- oreades Fisch., Mey. b.

- philadelphicus L. p.

:- spiculosus Hook., Arn. b. fr.

- uniflorus L. p.

Eritrichium californicum A. DC. a. - pedunculare A. DC. a.

Erodium litoreum Pers. a.

- malacoides Willd. a.

- Salzmanni Del. a.

- verbenifolium Del. a.

Erpetion reniforme Sweet. p. fr. " Erucastrum incautum Koeh. a.

Ervum Terronii Ten. a.

Erysimum Kunzeanum Boiss., Reut. a.

- sisymbrioides C.A.Mey. a.

Erythronium dens canis $\boldsymbol{L}$. p.

Eschscholtzia tenuifolia Bepth. $a_{\text {, }}$ Ethulia conyzoides $\boldsymbol{L}$. a. c.

Eucharidium concinuum Fisch,

$$
\text { Mey. a. }
$$

Euclidium syriacum $R$. Br. a.

Eudianthe laeta Rehb, a.

Eupatorium microstemum Cass. a. Euphorbia Esula L. (Amur.)

- maerorhiza C.A.Mey.p.

- pilosa L. p.

- procerit MIB. $\gamma$. tuberculata Koch. p.

Euryale ferox Salisb. p. c.

Euxolus caudatus Moq. $\beta$. gracilis Moq. a. c.

- lividus Moq. a. c.

- viridis Moq. a. c.
Falcaria dahurica $D C$. p.

Fedia graciliflora Fisch., Mey. a.

Felicia cotuloides DC. a.

- tenella Nees. a.

Ferulago sylvatica Rchb b.

Festuca Alopecuros Schoust. a.

- delicatula Lagasc. a.

- divaricata Desf. a.

- geniculata Willd. a.

- Myurus I. a.

- pectinella Del. a.

- procumbens $\boldsymbol{K}$ th. a.

- rigida $\boldsymbol{K} t h$. a.

Frankenia pulverulenta. $\boldsymbol{L}$. a. Fritillaria pallidiflora Sehrenk. p.

- ruthenica Wickstr. p.

Froelichia gracilis Moq. a. c.

- interrupta Moq. a. c.

Fuchsia arborescens Sims. var. syringiflora $\mathrm{Rgl}$. l. fr.

Fumaria agraria Lag. a.

- alexan Irina Ehrbg. a.

- capreolata $\boldsymbol{L}$. a.

- media Lois. a.

- micrantha Lag. a.

- muralis Sond. a.

Galatella dahurica DC. p.

- Hauptii Lindl. $\gamma$. tenuifolia Lull. p.

- punctata Lindl. $\eta$. discoidea Lall. p.

- rigida Cass. p.

Galium caudatum Boiss. a.

- divaricatum Lam, a.

- murale DC. a.

- nebulosum Boiss. a.

- parisiense L. $\alpha$. trichncarpum Tausch.a.

- - - $\beta$. lejocirpum Tausch. a.

- retrorsum DC. a.

- saccharatum All. a.

- tenuissimum $\boldsymbol{M I B}$. a.

- tricorne With, a.

- verticillatum Danth. a.

Gamolepis Tagetes $\boldsymbol{D C}$. a. c.

Gaştridium australe Beauy. a.

Gastrocotyle hispida Bgd. a.

Gastrolobium bilobum $\boldsymbol{R}, \boldsymbol{B r}$. 1. fr.

- cuneatun A. IIen/r. I, fr.

- spinosum Paxt. l. fr.

Gaudinia fragilis Beauv. a. 
Gaura Drummondii Torr., Gray. a. - parviflora Dougl, a.

- tripetala Cav. a.

Gaya simplex Gaud. p.

Gelasia viliosa Cass. p.

Genista canariensis $\boldsymbol{L}$. 1 . fr.

- ramosissima Webb. I. fr.

- Spachiana Webb. l. fr.

- tinctoria $L$. 1. fr.

Gentiana macrophylla Pall. p.

- Pneumonanthe L. p.

- septemfida Pall. p.

Geranium affine Ledeb. p.

- carolinianum $\boldsymbol{L}$. a.

- collinum Steph. a. glandulosum Ledeb. p.

- dissectum $\boldsymbol{L}$. a.

- divaricatum Ehrh. a.

- Londesii Fisch. p.

- Vlassowianum Fisch. p.

Geum brachypetalum Ser. p.

- intermedium Ehrh.a.brachypogon $C$. A. Mey. p.

- $\quad$ - $\quad$ - dolichopogon. C. A.Mey. p.

- macrophyllum Willd. p.

- rubellum Fisch., Mey. p.

- spurium Fisch., Mey. $\alpha$, urbano-strictum $\boldsymbol{C}$. $\boldsymbol{A}$. Mey. p.

- $\beta$. stricto-urbanum c. A. Mey. p.

- virginianum L. p.

- Willdenowii Fisch., Mey. p. Gilia coronopifolia Pers. b. fr.

- laciniata R., P. a.

- lutea Steud. a.

- - - P. aurea Rgl. a.

- millefoliata Fisch., Mey. a.

- multicaulis Benth. a. Gireoudia ricinifolia $\boldsymbol{K}$ l. p. c. Gloxinia speciosa Lodd. fl. erectis p. c.

- $\quad$ - $\quad$ - fl. pendulis p. c.

Gnaphalium cymatodes Kze. a. Goodia lotifolia Salisb. 1. fr. Grammanthes gentianoides DC.a.c. Grangea maderaspatana Lam. a. c. Grindelia inuloides Willd.1. fr.

- squarrosa Dun. p. fr.
Guizotia oleifera DC. a. c.

Günthera viscosa Ryl. a.

Gypsophila elegans $\boldsymbol{M B}$. a.

- muralis $\boldsymbol{L}$. a.

- perfoliata L. a. angustifolia Fenzl. p.

- viscosa Murr. a.

Haemanthus punicens $\boldsymbol{L}$. p. c.

Halleria lucida $\boldsymbol{L}$. 1 , fr.

Hasselquistia cordata $L$. fil. a.

Hebenstreitia dentata Thbg. a.

Hedysarum capitatum Desf. a.

- consanguineum $D C$. p.

- elongatum Fisch. p.

- flexuosum $L$. a.

- neglectum Ledeb. p.

- sibiricum Poir. p.

Heleninm tenuifolium $\boldsymbol{N u t t}$, a. e. Helianthemum salicifolium Pers. a.

- tauricum Rupr. a.

Helichrysum foetidum Mönch.b. fr. - semipapposum DC. 1 . fr.

Heliophila amplexicaulis $\boldsymbol{L}$. fil. a.

- crithmifolia Willd. a.

- longifolia DC. $\alpha$. glabra Rgl. a.

- pendula Willd. a.

- pilosa Lam. a. integrifolia DC. a.

- - - $\beta$. incisa DC. a.

- pusilla L. fil. a.

- sphaerostigma Kze. a.

- trifida Thbg. a.

Helipterum brachyrhynchum Sond. a.

Helosciadium leptophyllum $\boldsymbol{D C}$. a. Herniaria diandra Bge. a.

Herpestis chamaedryoides $\boldsymbol{H} . \boldsymbol{B}, \boldsymbol{K}$. a. c.

Heteracia Szovitsii Fisch., Mey. a. Heuchera americana $L, \alpha$. robusti Rgl, Rach.p.
$\beta$. gracilior Rgl.,Rach.p. $\gamma$.affinis $\boldsymbol{R g l}$.,

\section{Rach. p.}

- cylindrica Dougl. p.

- glabra Willd. p.

- hispida Pursh. p.

- micrantha Dougl. p.

- obtusissima Rgl., Rach. $\beta$. glabra Rgl., Rach. p. 
Heuchera pilosissima Fisch., Mey. p.

- pubescens Pursh. p.

Hieracium alpinum $\boldsymbol{L}, \boldsymbol{\alpha}$. genuinum Koch. p.

- cydoniaefolium vill. p.

- elatum Fries. p.

- gallicum Jord. p.

- glaucum All. p.

- incisum Hoppe. p.

- laciniosum Jord. p.

- laevigatum Willd. p.

- longifolium Schleich. p.

- medium Jord. p.

- murorum L. p.

- oreades Fries. p.

- prenanthoides Vill. p.

- $\quad-$ culatum Froel denti-

- rigens Jord. p.

- rigidum Hartm. p.

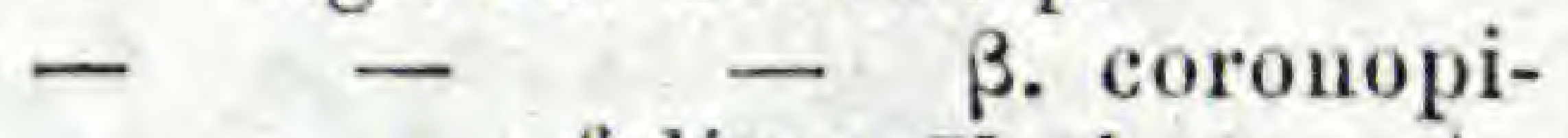

- sabauium L. p. folium Koch, 1 .

- Schmidtii Tausch. p:

- stoloniflorum $\boldsymbol{W}$., $\boldsymbol{K}$. p.

- vagum Jord. p.

- villosun Jacq. p.

Hippocrepis ciliata Willd. a.

- unisiliquosa L. a.

Hippophe rhamnoides $\boldsymbol{L}, 1$.

Holosteum umbellatum $L$. $\beta$. glandulosum a.

Hordeum hexastichum $\boldsymbol{L}$. a.

- jubatum $\boldsymbol{L}$. b.

- maritimum With. a.

- pratense Huds. p.

Hutehinsia alpina $\boldsymbol{R}$. Br. p.

Hyacinthus amethystinus $\boldsymbol{L}$. p.

Hymenophysa pubescens $\boldsymbol{C}, \boldsymbol{A}$. Mey. $\mathrm{p}$.

Hymenoxis californica $\boldsymbol{H o o k}$ a.

- mutica Torr., Gr. a.

Hyoscyamus orientalis $\boldsymbol{M} \boldsymbol{B}$. p.

Hyoseris microcephala Cass. a.

Hypecoum procumbens $\boldsymbol{L}$, $\boldsymbol{a}$.

Hypericum fimbriatum Lam. p.

Hypochaeris arachnoidea Poir. a. hispidis. a.

- Salzmanniana $D C$. a.

Jasione montana L. b, p.
Jasione montana L. $\beta$. major Koch. b. p.

Jasminum fruticans $L$. 1. fr,

Jasonia tuberosa $D C$. p. fr.

Iberis Lagascana DC.

- linifolia $L$. a.

Ilex-Aquifolium L. l. fr.

- balearica Desf. l. fr.

Impatiens glanduligera Royle. a. c.

- tricornis Lindl. a. c.

Iṇperatoria Ostruthium L. p.

Indigofera australis Willd. 1. fr.

Inula bifrons $\boldsymbol{L}$. var. tomentosa b. fr.

- glandulosa Willd.' p.

- grandiflora Willd. p.

- gravẹolens Desf. a.

- Helenium L. p.

- Lirta L. p.

- oculus Christi L. p.

- salicina Lin. p.

Johrenia dichotoma $D C$. b. fr.

Jonopsidium acaule Rchb. a.

Ipomoea sibirica Jacq. a,

Iris Güldenstädtiana Lepech. p.

- setosa Pall. p.

- sibirica L. $\boldsymbol{\beta}$. hatematophylla Fisch. p.

Isatis canescens $\boldsymbol{D C}$. b.

- oblongata $D C$. b.

- orientalis Willd.b.

- tinctoria L. b.

Isolepis Saviana $\boldsymbol{H}$., $\boldsymbol{S}$. a. c.

Isopyrum fumarioides $\boldsymbol{L}$. a.

Isotoma petraea $\boldsymbol{F}$. Mïll. a. c.

Juncus arcticus Willd. p.

- balticus Willd. p.

- compressus Jacq. p.

- filiformis $\boldsymbol{L}$. p.

- Gerardi Loisl. p.

- tritidus $\boldsymbol{L}$. p.

Juniperus excelsa $\boldsymbol{M I B}, 1$, fr.

- Sabina $\boldsymbol{L} .1$.

Jurinea alata $D C$. b. fr.

Jussiaea erecta $\boldsymbol{L}$. a. c.

Kennedya monophylla Vent. 1. fr.

- rubicundi Vent. 1. fr.

Knautia hybrida Coult.

- orientalis $\boldsymbol{L}$. a.

Knesebeckia discolor $\boldsymbol{K l}$. p, c.

- incarmata $K l$, 1. fr.

Knowltonia vesicatoria Sims. p. fr.

Kochia eriophora Schrad. a. 
Kochia hyssopifolia Noth, a.

Koeleria brachystachya. $D C$. a.

- laxa Lk. a.

- villosa Pers. a.

Koelpini, linearis Pall, $\alpha$, glabra DC. a.

Krubera leptophylla $\mathbf{H} / / / m$. a. Lactuca altaica Fisch., Mey. a.

Lagurus ovatus $\boldsymbol{L}$. a.

Lallemantia canescens Mey. a.

- iberica Fisch., Mey. a.

- peltata Fisch., Mey. a.

Lantana alba Mill. l. c.

Lappago racemosa Willd. a.

Laserpitiu. hispidum $\boldsymbol{M B}$, p. .

- Siler. L. p.

Lasiagrostis splendeus $\boldsymbol{K} t \boldsymbol{h}$. p.

Lasiospermum pedunculare. Lagasc, p. fr.

Lasthenia glaberrima $D C$. a,

Lathyrus mexicanus Sehlchtd, a.

- Nissolia L. a.

- pisiformis $\boldsymbol{L}$. p.

- purpureus Desf. a.

- Sativus L. a.

- sphaericus Retz. a.

Laurentia Michelii $\boldsymbol{D C}$. a.

Laturus nobilis $\boldsymbol{L}$. l. fr.

Lavatera arborea $\boldsymbol{L}$. 1 . fr.

- Olbia L, 1. fr.

- thuringiaca. L. p.

Leontice altaica Pall. p.

Leontodon caucasicus Fisch. p.

- - $\quad$ - $\beta$. hispidissimus $\mathrm{p}$. hastilis $\boldsymbol{L} . \boldsymbol{\alpha}$, vulgaris Koch. p.

Koch. p.

- Rosani DC. p.

Leonurus Cardiaca $\boldsymbol{L}$. p.

- $\quad$ - $\beta$. villosus

Benth. p.

- glaucescens Bge. p.

Lepitium amplexicaule Willd. p.

- cordatum Willd. p.

- latifolium $\boldsymbol{L}$. $\alpha$. glabrum Ledeb. p.

Leptospermum baccatum $\mathrm{Sm}$.

1. fr.

- flavescens Sm. l. fr.

- scoparium $S m$. 1. fr.
Leptospermum scoparium Sm. $\alpha$. linifolium $D C$. I. fr.

- stellatum Cav. 1. fr.

Lepturus incurvatus Trin. a.

- subulatus ith. a.

Lepyrodicles holosteoides Fenzl. a.

Lespedeza juncea Pers. 1. (Amur.) Leucanthemum sibiricum $D C$. $\beta$. acutilobum DC. p.

Leucas urticaefolia $\boldsymbol{R}$. $\boldsymbol{B r}$. a. c.

Leuceria senecioides Hook,, Arn. a.

arachnoidea $\boldsymbol{R} \overline{y l}$. a.

Leyssera capillifolia $D C$, a.

Liatris spicata Willd. p.

Libanotis condensata Fisch., Mey.p.

Livertia formosa Grah. p. fr.

Ligularia macrophylla Ledeb. p.

- sibirica Cass. p.

- speciosa Fisch, Mey. p.

Ligusticum alatum $\boldsymbol{S p r}$. p.

- $\operatorname{scoticum} \boldsymbol{L}$. p.

- Seguieri Koch. p.

Limnanthes alba Hartw, a.

- Douglasii R.Br.a.

- rosea Hartw. a.

Limnocharis Plumieri Rich, p. c.

Linaria albifrons $S p r$. a.

- amethystea $\boldsymbol{L} \boldsymbol{k}, \boldsymbol{H} / \mathrm{/m}$. a.

- aparinoides Chav. a.

- bipartita Willd, a.

Rgl. a.

- Boissieri Walp. a.

- chalepensis Mill. a.

- cirrhosa Willd. a.

- Lirta Moench. a.

- litoralis Willd, a.

- macropoda Boiss, a.

- praetermissa Delat. a.

- Salzmanni Boiss. $\alpha$. viulacea Boiss. a.

- saxatilis $L k$., $\boldsymbol{H} / \mathrm{f} \boldsymbol{m}$, a.

- - chav. a. $-\beta$. stricta

- spicata Kze. a.

- spuria Mill. a.

- supina Desf. a.

Bgl. a.

var. violacea

- triornithophora Willd. b. fr.

triphylla Willd. a. 
Linaria versicolor Noench, a.

Lindheimera texana Gray., Engelm. a.

Linum angustifolium Inuds. a. Liriodendron tulipifera $\boldsymbol{L}, \mathrm{l}$, fro Lithospermum latifolium Michx. p.

- officinale $L$. p.

- tenuiflorum $\boldsymbol{L}$. fil. a.

Lobelia heterophylla Lab, a. c.

- ocimoides Kze, a.

- sessilifolia Lam. p. (Amur.)

- xalapensis $\boldsymbol{H}$.. B. a.

Loefflingia hispanica $\boldsymbol{L}$, a.

Logfia gallica Coss., Germ. a.

Lolium scabrum Presl, a.

- temulentum $\boldsymbol{L}$. a. Koch. a.

Lonicera tatarica $\boldsymbol{L}$. 1 .

- $\beta$. speciosim

- - - fl. rubro. I.

- Xylosteum L. 1.

Lopezia coronata Andr. a.

Lotus diffusus Soland. a.

- filicaulis Dur. a.

- gracilis $\boldsymbol{W}, \boldsymbol{K}$. a.

- hispidus Desf. a.

- ornithopodioides $\boldsymbol{L}$. a.

Lupinus albus $\boldsymbol{L}$. a.

- angustifolius $\boldsymbol{L}$. a.

- aridus Dougl. a.

- Barkeri Lindl. a,

- Hartwegii Lindl. a.

- linifolius Roth. a.

- nanus Dougl. a.

- nootkatensis Don, p.

- pilosus $\boldsymbol{L}$, a.

1- pubescens Benth. a.

Luzula spicata Desv. p.

Lychnis chalcedonica $\boldsymbol{L}$. $\beta$. dubia Lall. p.

Lycopsis arvensis $L$. a.

- variegata $L$. a.

Lyperia diandra E. Mey. a.

- violacea Benth. a.

Lysimachia punctata Jucq. p.

Madaria corymbosa DC. a,

- elegans $D C$. a,

Madia racemosa Torr., Gray, a.

- sativa Molina. a.

- stellata Fisch., Mey. a.

Malva capensis $L$. 1 . fr.

- moschata L. a.
Malva nicaeensis All, a:

- pulchella Bernh. a.

Malvastrum coromandelianum

Garke, a. c.

- tricuspidatum Gray. a. c.

Mammillaria columnaris Mart. l. c.

- crassispina Pfr. 1. c.

- dolichocentra Lem. 1. c.

- fulvispina Haw. 1. c.

$$
\text { - Salm. - 1. c. }
$$

- multiceps Salm. 1. c.

- polythele Mart. 1. c.

- $\beta$. quadrispinaSalm.1.c.

- - - - r.latimamma Salm. l.c.

- pusilla DC. 1. c.

- tetracentra H. Berol. I, c.

Manulea Cheiranthus $\mathbf{L}$. a.

Maruta fuscata $D C$. a.

Matricaria microcephala Rupr. a.

Matthiola parviflora R. Br. a.

- tricuspidata, R, Br, a.

Mazus rugosus Lour, a. c.

Medicago glutinosa $M B$, p.

Melaleuca hypericifolia $\boldsymbol{S} \boldsymbol{m}$. 1. fr.

Melampodium divaricatum $\boldsymbol{D C}$. a.c.

Melandryum brachypetalum Fenzl. p.

- pratense Roehl. b.

- soongaricum Fisch., Mey. p.

Melia Azedarach $\boldsymbol{L}$. 1 . fr.

Melilotus alba Desr. b.

- macrorhiza P'ers. $\beta$. palustris Koch. b.

Meniocus linifolius $D C$. a.

Mercurialis violaefolia Kze. a. c.

Merendera soboliferaFisch.,Mey.p.

Mespilus pyracantha $\boldsymbol{L}$. l. fr.

Micropus er ectus $\boldsymbol{L}$. a. "

Microsciadi um tenuifolium Boiss. a.

Microtea de bilis Sweet, a. c.

Milium vernale $\boldsymbol{M B}$. a.

Mitella diphylla $\boldsymbol{L}$. p.

Mitellopsis Drummondii Meisn. p. Mioehringia pentandra Gay. a.

Mollugo verticillata $\boldsymbol{L}$. a.

Molopospermum cicutarium LC. p.

Moeuchia quaternella Ekrh. a,

Monolopia glabrata Fisch, Mey. a . Moricandia Ramburii $\vec{v} e b$, b. fr. 
Moseharia pi nuatifida $\boldsymbol{R}, \boldsymbol{P}$, a. Moschosma polystachyum Benth. a. c.

Myagrum perfoliatum $L$. a. Myosotis azorica Wats. p. fr. Myrica Faya dit. 1. fr.

\section{- Gale L. 1.}

Navarrettia involuerata $\boldsymbol{R}$, $\boldsymbol{P}$. a.

Nemesia floribunda Lehm. a.

- versicolor E. Mey. a. coerulea $\mathrm{Rgl}$. a.

Nepeta botryoides Ait. a.

- macrantha Fisch. p.

- nuda Lin. p.

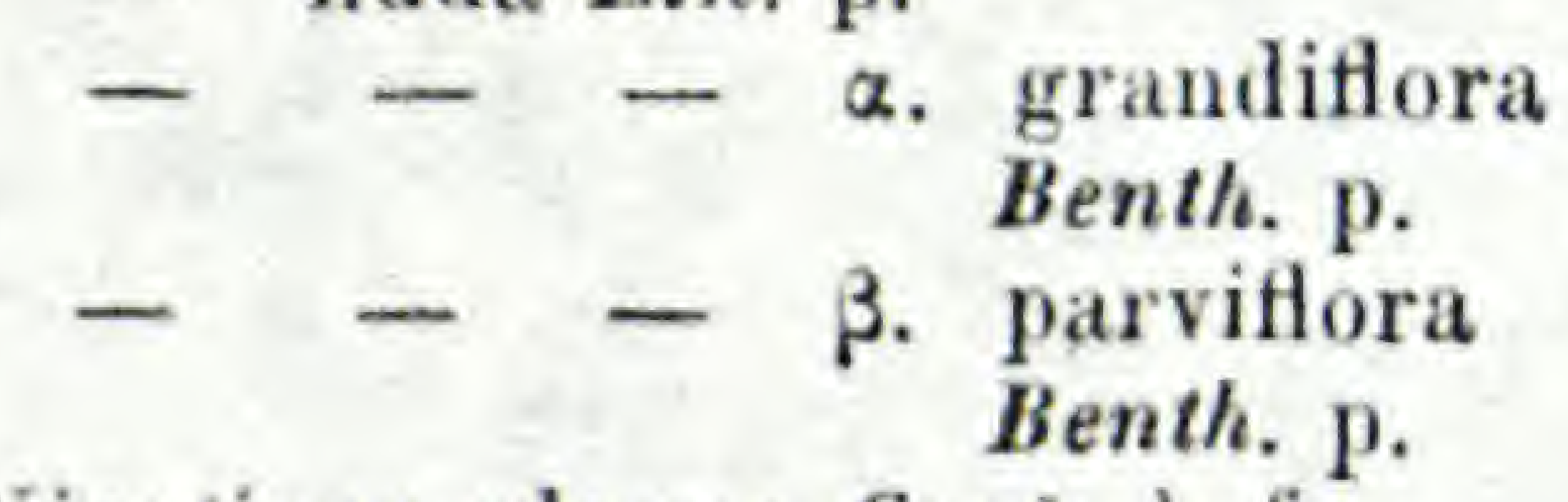

Nicotiana glauca Grah. I. fr.

Nolana atriplicifolia $\boldsymbol{D}$. Don. a. - prostrata $\boldsymbol{L}$. a.

Nonnea alba $\boldsymbol{D C}$. a.

- flavescens C. A. Mey. a.

- nigricans $\boldsymbol{D C}$. .

- rosea Fisch., Mey. a.

Nothoscordium fragrans Kth. p. fr.

- striatum $\boldsymbol{K}$ th. p. fr.

Notobasis syriaca Cass. a.

Nycterinia selaginoides Benth. a.

Nymphaea alba $\boldsymbol{L}$. p.

- amazonum Mart., Zucc. p. c.

- coerulea Savign. p. c.

- dentata Schum., Thon. p. c.

- odorata. Ait. p. e.

- scutifolia DC. p. c.

- thermalis DC. p. c.

Ochtodium aegyptiacum $\boldsymbol{D C}$. a.

Ocinum canum Sims. a. c.

- carnosum Lk., Otto. 1. c.

Oenothera Cavanillesii Spach. a.

- concinna Don. a.

- cruciata Nutt. b.

- densiflora Lindl. a.

- Fraseri Pursh. p.

- linifolia Nutt. a

- propinqua Spach. a.

- sinuata $\boldsymbol{L}$. a.

- stricta Ledeb. a.

- tetraptera Cav. a.

Oplismenus Crus galli $\boldsymbol{k} t \boldsymbol{h}$, a.

Oreomyrrhis erioporła Endl. p. fr.

Orlaya platycarpa Koch. a.
Ornithogalum refractum Kit. p.

- sulphureum $\boldsymbol{R}, \boldsymbol{S}$. p. fr.

Orobus atropurpureus Desf. 3 . unijugus Fisch. a.

- vernus $\boldsymbol{L} . p$.

- - - B. flaccidus Koch.

Oryza sativa $\boldsymbol{L}$. aristata. a. c.

Oxalis rosea Jucq. a.

- villosa $\boldsymbol{M B}$. p.

Oxytropis glabra $D C$. p.

Ozothamnus diosmaefolius DC.1. fr.

Pachypodium erysimoides Webb., Berth. a.

Paederota Ageria $\boldsymbol{L}$. p.

Paeonia anomala $\boldsymbol{L}$. carpell. pubescentibus $p$.

- arietina Anders. $\beta$. Andersonii p.

- decora Anders. p.

- humilis Retz. p.

- lanceolata Salm. p.

- peregrina Mill. $\gamma$. fulgens p.

- pubens Sims. p.

Paliurus aculeata Lam. 1. fr.

Panicum aegyptiacum Retz. a.

- ciliare Retz. a.

- colonum L. a. c.

- eriogonum Schrad. a.

- eruciforme Sibth., Sm, a.

- fimbriatum $\boldsymbol{k} t \boldsymbol{h}$, a.

- glabrum Gaud. a.

Papaver alpinum $\boldsymbol{L}$. $\alpha$, nudicaule Fisch., Hey. p.

- amoenum Lindl. a.

- caucasicum MB. capsulis oblongis glabris b.

- MB. capsulis oblongis hispidis b.

- capsulis subglobosis hispidis $b$.

- hybridum $\boldsymbol{L}$. a.

- orieutale $\boldsymbol{L}$. p.

- rupifragum Boiss., Reut. a.

- setigerum $\boldsymbol{D C}$. $\beta$. muticum Rgl. a.

Parietaria micrantha Ledeb, a.

Paronychia echinata Lam. a.

Passiffora suberosa $L$. l. c.

Paulownia imperialis Sieb, Zucc. I. fr.

Pelargonium australe Jacq. a.

Pennisetum cenchroides Rich. a. c. 
Peplis biflora Saltzm, a.

Peucedanum austriacum Koch, p.

- officinale L. p.

- Oreoselinum Moench. p.

- rablense Koch. p.

Phaca frigida L. 3. americana Hook. p.

Phacocapnos Cracca Bern. a. ci

Phalaris paradoxa L. fil. a.

- truncata Guss. a.

Phillyraea latifolia L. 1. fr.

Phlomis alpina Pall. p.

Phyllanthus cantoniensis Horn. a.c.

- juglandifolius Willa. l. c.

- obovatus Muehlbg. a. c. ‘

- ussuriensis Rupr. a. c.

Physalis hirsuta Dun. $\gamma$. barbadensis Dun. a. c.

Physolophium saxatile Turcz. p, Physostegia virginiana Benth. p, Phyteuma Michelii Bertol. $\alpha$. betonicaefolium Koch. p.

- Scheuchzeri All. p?

Phytolacca decandra $\boldsymbol{L}$. p. fr.

- octandra L. p. fr.

Picridium intermedium Schultz. Bip. a.

- tingitanum $\boldsymbol{L}$. a.

- vulgare Desf. a.

Pimelea clavata Lab. 1 . fr.

- drupacea Lab. l. fr.

Pinus alba Ait. 1.

- Cembra L. 1.

- $\quad$ - $\quad$ - $\beta$. pumila Pall. 1.

- davurica Fisch. 1.

- Laricio Poir. c. Pallasiana Endl. 1.

- Larix L. 1.

- $\quad$ - $\beta$. pendula Endt. 1.

- Ledebourii Endl. 1.

- microcarpa Lamb. 1.

- nigra Ait. l.

- Nordmanniana Stev. 1. fr.

- obovata Ledeb. 1.

- pendula Sol. I.

- Pichta Fisch. 1.

- Pinea L. l.

- Strobus L. 1.

- sylvestris L. I.

Pircunia esculenta Mog. p. fr.

Pistacia mutica Fisch., Mey. l. fr.

Pisum maritimum L. p.
Plagiobothrys rufescens Fisch., Mey. a.

Plantago altissima $\boldsymbol{L}, \mathrm{p}$.

- aristata Michx, a.

- Cornuti Gouan. p.

- Cynops L. p.

- Durvillei Del. p.

- - - californica Fisch., Mey. p.

- Hookeriana Fisch., Mey. a.

- Lagopus L. a.

- lanceolata L. p.

- maritima L. p.

- mexicana $\boldsymbol{L k}$. a.

- microcephala Poir. a.

- montana Lam. p.

- Psyllium L. a.

- - - - - divaricata.a.

- salsa.MA. p.

- saxatilis MB. p.

- serpentina Lam. p.

- Serraria L. a,

- tenuiflora W., K. a.

- tumida Llk. a.

- virginica $L$. $\beta$. a.

Platanus occidentalis $\boldsymbol{L}: \mathbf{l}$.

Platycodon grandiflorum A. DC. p. (Amur.)

Platylobium triangulare $\boldsymbol{R} . \mathbf{B r}$. l. fr. Platy theca galioiles Steotz. 1. fr. Plectranthus australis $\boldsymbol{R}$. Br. l. c. - parviflorus Willd. 1. c.

Plectritis congesta $D C$, a. .

Plumbago micrantha Ledeb. a.

Poa alpina $L$. p.

- caesia $S m . p$.

- ciliaris $\boldsymbol{L}$. a.

- megastachya Koelqr. a.

- mexicana Lag. a.

- nemoralis $\boldsymbol{L}$. v. glaucescens Anderss. p.

- peruviana Jacq. a.

- rufescens Schrad. a.

- serotina Ehrh. p. ,

- virescens $\mathrm{K} t h$. a,

Pocockia cretica Ser. a.

Podolepis chrysantha Endl. a. c.

- gracilis Grah. a. c.

Podotheca gnaphalioidesGrah.a.c.

Polanisia graveolens $\boldsymbol{D C}$. a, c.

- trachysperma Torr, Gr. a. C. 
Polycalymna Stuartii Mill, Sonder. a. c.

Polycarena capensis Benth. a.

Polycarpaea latifolia Poir. a. c.

Polycarpon tetraphyllum $\boldsymbol{L}$. fil. a.

DC. a.

3. diphyllum

PolyceuiahebenstreitioidesChois. a.

Polyenemum majus A. Br. a.

Polygala cordifolia $r h b g$. 1 . fr.

Polygonum alpestre C. A. Mey. p.

- alpinum All. p.

- divaricatum $\boldsymbol{L}$. p.

\section{- - - - s. subsca- bridum Ledeb. p.}

- salignum Willd. p.

Polypognn litoralis Sm. a.

- noritimus Willd. a.

- monspeliensis Desf. $\alpha$. major

Kth. a.

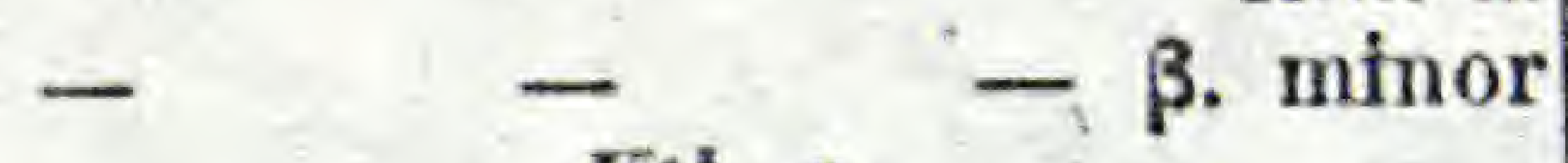

Kth. a.

Portulaca rostellata Brign. a. c.

Potentilla aprica Rupr. p.

- atrosanguinea Don. p.

- collina Wib. p.

- De Tommasii Ten. p.

- digitato - flabellata A. Br., Bouché. p.

- gariepensis Eeklon. a.

- Goldbachii Rupr. b.

tosa b.

- insignis Royle. p.

- kurdica Boiss., Hohenark. p.

- megalontodon Lehm. p.

- millegrana Engelm. a.

- multifida $\boldsymbol{L}$. p.

- norvegica $\boldsymbol{L}$. b.

- $\quad$ - B. hirsuta Lehm. b.

- obscura Willd. p.

- pulcherrima Lehm. p.

- recta $\boldsymbol{L}$. p.

- rupestris $\boldsymbol{L}$. p.

- Sadleri Rehb. p.

- supina L. a.

- verna L. $\beta$ crocea Koch. p.

- villosa Pall. p.

- viscosa Don. p.

Poterium dictyocarpum Spach. $\beta$. glaucum Spach. p.

- muricatum Spaeh. p.
Primula mistassinica Micks. p.

- officinalis .Jacq. $\beta$. inflata Ledeb.

Prostanthera Lasianthos Lab. 1. fr. Psidium litorale Raddi. l. c.

Psilonema calycinum Fisch., Mey. a.

Psilurus nardoides Trin. a.

Psychine stylosa Desf. $\beta$. longibracteata $\mathrm{Rgl}$. a.

Pterocephalus plumosus Coult. a.

Pteroneuron graecum DC. a.

Pteropogon chilense Fisch, Mey. $\alpha$. majus Fisch., Mey. a.

\& chilense Fisch., Mey. $\beta$. minus Fisch., Mey. a.

- pygmaeum $\boldsymbol{D C}$. a.

Pterostegia drymarioides Fisch., Mey. a. c.

Ptychotis ammoides Koch. a.

Pulicaria sicula Moris. a.

Pultenaea retusa $S m$. 1. fr.

- tenuifolia R. Br. l. fr.

Punica Granatum $\boldsymbol{L}$. l. fr.

Puschkinia scilloides Adams. p.

Pyrethrum ambiguum Ledeb. p.

- caucasicum Willd. p.

- macrophyllum Willd. p.

Pyrus Aria Ehrh. 1.

- Aucuparia Gaertn. fruct. luteis 1.

- baccata $L .1$.

- cerasifera Tausch. 1.

Tausch. 1.

- prunifolia Willd. 1.

Quercus Ilex L. I. fr.

Ranunculus abortivus $\boldsymbol{L}$. p.

- lomatocarpus Fisch., Mey. a.

- muricatus $\boldsymbol{L}$. a.

- nodiflorus $\boldsymbol{L}$. a.

- ophioglossifolius Vill. a.

- parviflorus $\boldsymbol{L}$. a.

- Philonotis Ehrh. a.

${ }_{\text {dius }} \overrightarrow{D C}$. a. $^{-}$interme-

- Schraderianus Fisch., Mey. a.

- trachycarpus Fisch., Mey. a.

- trilobus Desf. a.

Rapistrum rugosum All. a.

$$
\text { glabris a. }
$$

Reseda complicata Bory. a. 
Reseda inodora Rohb. a. - truncata Fisch., Mey. b. Rhamnus Alaternus I. 1. fr. - catharticus $\boldsymbol{L} .1$.

Rhaponticun cynaroides Less. p. - pulchrum Fisch., .Hey. p.

- scariosum Lam. p. DC. p.

- B. lyritum

Rhodanthe Manglesii Lindl. a. c. Rhynchopsidium sessiliflorum $D C$. a.

Ribes aciculare $S m .1$.

Richardia scabra $\boldsymbol{L}$. a. c. Rochelia leiocarpa Ledeb. a. - stellulata Rchl. a.

Rosa pimpinellifolia $L$. 1 .

Rostellularia abyssinica Brongn.a' c. Rudbeckia laciniata $\boldsymbol{L}$. p.

- speciosa Wender. p.

Rulingia corylifolia Grah. 1. fr. Rumex alpinus $\boldsymbol{L}$. p.

- aquaticus $\boldsymbol{L}$. p.

- brasiliensis $L k$. a.

- bucephalophoras $\boldsymbol{L}$. a.

- confertus Willd. p.

- conglomeratus Murr. $p$.

- crispus L. p.

- Gmelini Turcz. p.

- hamatus Trevir. p.

- Hyilrolapathum Huds. p.

- Kimthiamus Campil. p.

- Marschallianus Rchb. a.

- maximus Schreb. p.

- obtusifolins $\boldsymbol{L}$. 1.

- pratensis Mert., Foch. p.

- soongaricus Schrenk. it.

- strictus $L k$. a.

- ucranicus Fisch. at.

- verticillatus $\boldsymbol{L}$. p.

- vesicarius $L$. a.

Sagina apetala $\boldsymbol{L}$. a.

- - - - glabía a. j

- ciliata Fr. a.

- maritima Don. Rchb. a.

- saxatilis Wimm. a.

- stricta Fries. a.

- subulata Wimn. a.

Salvia aethiopis $\operatorname{Lin}$. b. fr.

- algeriensis Desf. a.

- argentea Silith. b. fir.

- hispanica L. a.
Salvia Horminum $L$. a.

- lanceolata Brouss. at.

- porphyrantha Decsne. 1. fi.

- rhombifolia R., P. a.

- runcinata L. fil. I. fr.

- Sibthorpii Smith, p.

- Spielmanni Seop. a.

- spinosa $L$. a.

Sambucus nigra $L$. I.

- racemosa $\boldsymbol{L}$. 1 .

Sanguisorba alpina Bge.p.

- pulchella C. A. Mey.

- - - - flor roseis p.

- tenuifolia Fisch. $\alpha . \quad C$. A. Mey. p.

- - - - rirhodantha C. A. Mey. p.

Sanvitalia procumbens Lam. a. Saponaria calabrica Guss. a.

- cerastoides Fisch. a.

- orientalis $\boldsymbol{L}$. a.

- porrigens $\boldsymbol{L}$. a.

Saussurea amurensis Turcz, p.

- elongata DC. $\beta$. polypodifolia $D C$. $p$.

Saxifraga aizoi les $\boldsymbol{L}$. p.

- Aizoon. Jacq. p.

- bronchialis L. B. Ledeb. p.

- caespitosa L. $\alpha$. compacta

Koch.p.

- $\alpha$. compacta Koch.pilosap.

- B. laxa koch. p.

- B., laxa Kork. pilosit 1 .

- controversa Sternby. a.

- Geum L. $\delta$. Donii Sternbg. p.

- Lirsuta L. 1.

- Hostii Tausch. p.

- hypuoides L. p.

- muscoides Wulf $\beta$ intermedia hoch:p.

- mutata Lin. 1.

- nivalis Lin. p.

- orientalis Jacq. a.

- petraea L. :1.

- rotundifolir L. p.

- stellaris $\boldsymbol{L}$. p.

- tridactylites $\boldsymbol{r}$. a.

Scabiosa caucasica MIB. p.

- Fischeri DC. p. 
Seabiosa maritima L. b.

- prolifera $\boldsymbol{L}$. a.

seandix brachycarpa liuss, a.

- hispanica Boiss. a.

- iterica $\boldsymbol{M B}$.

- macrorlyucha Fisch, Mey. a.

- P'ecten Veneris $\boldsymbol{L}$. a.

- persica Mart. a.

- pinnatitida Vent. at.

- $\beta$. soongarica Schrenk a.

Schist: nthe peduncularis Kze. a. fr. Schizanthus pinnatus $\boldsymbol{R}$., $\boldsymbol{P}$. a.

\section{nus Rigl. a.}

ß. lilaci-

schizopetalum Waiker Sims, a.

Scilla cernua Red, $\alpha$. paticiovulata Rigl. $\mathrm{p}$.

- pratensis W., h. p.

serophularia divaricata ledb p.

- Neesii Wirtg. p.

- nodosa $\boldsymbol{L}$. p.

- peregrina $\boldsymbol{l}$. a.

- Scopolii Hoppe. p.

Scutellaria alpina $\boldsymbol{L}$. p.

$$
\text { - }- \text { - } \beta \text {. Iupulina }
$$

- commutata Guss. p.

sidum Aizoon L. p.

- Anacampseros L. p.

- annuum $\boldsymbol{L}$. a.

- Cepaea $\boldsymbol{L}$. $\beta$.gaiioides DC. a.

- $\quad$ - $\delta$. tetraphyllum DC. a.

- coeruleum Vahl, a.

- Ewersii Ledeb. p.

- hybridum L. p.

- involucratum MB. P.

- kantschaticum Fisch. p.

- leptorhizum C. A. Mey. p.

- maximum Suter. p.

- oppositifolium Sims. p.

- pallidum $\boldsymbol{M B}$. . 1 .

- purpurasceus Koch. p.

- Rhodiola DC. p.

- _ - - .viride Rgl.p.

- saxatile Wigg. a.

- stellatum $\boldsymbol{L}$. a.

belinum Carvifolia $\boldsymbol{L}$. p.

Senebiera linoides $\boldsymbol{D C}$. a.

benecio arabicus $\boldsymbol{L}$. a.
Senecio cordatus koch. p.

- inaequidens DC. a.

- lividus $\boldsymbol{L}$. it.

- macrophyllus MB. p.

- nebrodensis $L$ : a.

- octogiossus DC. p.

- palmattis Pall. p.

Serratula centauroides $L$. p.

- coronati L. p.

- glauca Ledeb. p.

- radiata $\boldsymbol{M B}$. p.

Sibbaldia cuneata Edgew. p.

Sideritis lanata $\boldsymbol{L}$. a.

- montana $L$. a.

Siegesbeckia flosculosa Herit. i. e.

- orientalis $\boldsymbol{L}$. a. c.

Sieversia montana Spr. p.

Silaus carvifolius C. A. Mey. p.

Silene alpestris Jacq. p.

- ambigua Camb. a.

- annulata Thore. a.

- timbriati Sims. p.

- graminifolia Oth. p.

- maritima With. p.

- melandryiformis Maxim. L. (Amur.)

- noctiflora $\boldsymbol{L}$. a.

- nutans L. var. glaberrima p.

- orientalis Nill. b. fr.

- pelidua Rchb. p.

- repens Patr. p.

- Saxifraga L. p.

- velutina Pourr. 1. fr.

- - - florib. rubicundis 1. fr.

Zawadskii Herbich. p.

Silphium laciniatum $\boldsymbol{L}$. p.

Silybum marianum Gärtn. vireus. a.

Sinapis abyssinica $\mathbf{A}$. Br. a.

- Allionii Jacq. a.

- Cheiranthus Koch. $\beta$. cheiranthiflora Gren., Godr.it.

- hispida Schousb. a.

- juncea $\boldsymbol{L}$. a.

- - var. pilosa a.

- integrifolia Willd. a.

Sisymbrium brachypetalum Fisch., Mey. a.

- bursifolium $\boldsymbol{L}$. a.

- canescens Nutt. a.

- Columnae L. a.

- contortuplicatum DC, a. 
Sisymbrimm C'uningianum Fisch,

$$
\text { Mey. a. }
$$

- hirsutum DC. a

- Irio L. a.

- nanum DC. $\beta$. integrifolium Rgl. a.

- nitidum Zea. a.

- persicum $\boldsymbol{S p r}$. a.

- polyceratum $\boldsymbol{L}$. a.

- pumilum Stephan. a.

- salsugineum Pall. a.

- Sophia L. a.

- subhastatum Lagasf. a. ;

- tripinnatum DC. d.

Sisyrinchium micranthum C'av. a. solidago canadensis $\boldsymbol{L}$. p.

$$
\text { - }-\gamma \text { procera }
$$

- glabra Desf. p.

- hirta Willd. p.

- humilis Herb. Banks, p.

Soliva anthemifolia $R$. Br. at.

- Barklayana DC. a.

- Sessilifolia $\boldsymbol{R} ., \boldsymbol{P}$. a.

Sophora japonica $L$. I. fr.

Sorghum halepense Pers. it. c.

Spartium junceum $\boldsymbol{L}$. 1. fr.

- scoparium L. 1, fr. "

Specularia biflora Fisch, Mey, it.

- falcata A. DC. a.

- hybrida A. DC. a.

- pentagonia A. DC. al.

- perfoliata A.DC. a.

sphaerolobium fornicatum Benth. 1. fr.

Sphaerostigma Chamissonis Fisch., Mey. a.

- micranthum Walp, a.

- minutiflorum Fisch.p Mey. a.

- strigùlosum Fisch., Mey. a.

Sphenandra viscosa Benth. a.

Sphenogyne adonidifolia $\boldsymbol{D C}$. a.

- anthemoides R. Br: a.

- microcephala DC. a.

- speciosa Maund, a.

Spiraea amurensis Maxim. (Amur) 1.

- carpinifolia Willd. 1.

- chamaedrifolia L. 1.

- contusa Rgl., Kcke. I.

- tilipendula $\boldsymbol{L}$. p.

- laevigata Lin. I.

- opulifolia L. I.
Spiratea salicifolia $\boldsymbol{L} .1$.

- sorbifolia $\boldsymbol{L}$. 1 .

- tomentosia L. 1.

Stachys alpina $\boldsymbol{L}$. $\beta$. intermedia Benth. p.

- recta L. p.

- setifera C. A. Mey. p.

Statice speciosa $\boldsymbol{L}$. P.

- tomentella Boiss, p.

Stellaria borealis Hook. p.

- viscida $\boldsymbol{M B}$, a.

Stenactis ammua Cass. b.

Stephanophysum brevifiglium Pohl. 1. c.

Stevia confertu DC, $\alpha$. pilosa DC. p. fr.

Sterculia platonitolia $\boldsymbol{L}$. fil. 1. fr.

stipa tortilis Desf. a.

Streptocarpus Rhexii Lindl, p. c.

Stylidium adnatum $\boldsymbol{R}$. Br. p. fr.

Suaeda altissima Pall. as:

- gracilis Moq. a.

Succisat pratensis ..oench. p.

Succovia balearica Medic. a.

Sutherlandia frutescens $\boldsymbol{R} . \boldsymbol{B r}$. 1. fr.

Synedrella noditlora Gärtn. a. c.

Syringa Josikatea Jacq.fil. 1.

T'anacetum Achillea Schultz. Bip.

p.

- atratum Schultz. Bip. p.

- Balsamita L. p...

- boreale Fisch. p.

- carneum Schultz. Bip. p.

- corymbosum Schultz. Bip. p.

- Leucanthemmm Schultz. Bip.

a. vulgure Rchb, p.

Taraxacum Stevenii $D C$. P.

Telanthera Meyeriana Kyl., Kcke. 1. c.

Teloxis aristata Moq. a.

Tetrapoma harbareaefolitim Turcz. p.

Tetratheca epilobioides Steetz. $\beta$. hirsuta Sttetz. 1. fir.

Teucrium lamiifolium Urv. b. fr.

Thalictrum angustifolium Jacq. p.

serpitifolium Koch. p. aquilegifolium $\boldsymbol{L}, \mathrm{P}$.

$\therefore \beta$ atro-

purpureuni foch. $p$.

- elatum Jacq. 1 . 
Thatictrum foetidum $\boldsymbol{L} . \beta$. glabrum hoch. p.

- glaucum Desf. p.

- Jacquinianum Koeh. p.

- kemense Fries. p.

- nedium Jacq. p.

- minus L. p.

- $\quad-\quad$ - Koch. p.

- mucronatum Ledeb. p.

- nutans Desf. p.

- rugosim Ait. p.

- simplex $\boldsymbol{L}$. p.

- $\quad$ - $\quad$ -. s. rictum Rgl.p.

- Trigynum Fisch. p.

Thertuopsis fibacea $\boldsymbol{D C}$. p.

Thisantha glomeratit Eekl., Zeyh. a. c.

- patens Eckl, Zeyh. a. c.

Thomasia purpurea Gay. 1. fr.

Tillaea connata $\boldsymbol{R}$, $\boldsymbol{P}$. a.

Tordyli a maximum $\boldsymbol{L}$. a.

- syriacum $L$. a.

Torilis nodosa Gärtn, a.

'Tournefortia heliotropioides Hook. 1. fr.

Tradescantia crassula Lk., Otto. p. c.

- erecta Jacq. a. c.

- undatal Willd. a. c.

Tragopogon parviflorus Horn. b.

- porrifolias $\boldsymbol{L}$. b.

Trichodesma indicum Lehm. a. c.

Tricuogyne verticillata Less. a.

Trifolium alpestre $\boldsymbol{L}$. p.

- canescens Willd. p.

- trichocephalum $\boldsymbol{M B}$. p.

'Trisetum myi ianthum Fisch., Mey. a.

- neglectum $\boldsymbol{R}, \boldsymbol{S}$. a.

Triticum ciliatum $\boldsymbol{D C}$. a.

- orient le $\boldsymbol{M B}$. a.

- prostratum L. fil, it.

- squarrosam Foth. a.

- subtile Fisch., Mey. a.

- villosum Beauv. a.

Trollius altaicus C. A. Mey. p.

- europiteus $\boldsymbol{L} . p$

$$
\begin{aligned}
& \text { - } \beta \text {. humilis } \boldsymbol{D C} \text {. } \\
& \text { - pedunculatus p. } \\
& -\quad \text {. uapellifolius } \\
& \text { DC. p. }
\end{aligned}
$$

- patulus Salisb, var. causasicus involueratus $\boldsymbol{R} g l$.p.
Trollius patulus Salisb. var. cattcasicus pedunculatus Rgl. p.

Tropieolum tricolorum Sweet.p. fir.

Tunica prolifera Scop. a.

- Saxifraga Scop. a.

- velutina Fisch., Mey. a.

Turritis glabra Lin. b.

Uropetalum serotinum Gavl. $\beta$. $\boldsymbol{k}$., S. p.

Urtica Dodartii $L$. a.

- membranacea Poir. a.

- pilulifera $\boldsymbol{L}$. a.

- stachydifolia Kth., Bouche...

Vaillantia hispida $\boldsymbol{L}$. a.

- muralis $\boldsymbol{L}$. a.

Valeriana alliariaefolia vahl.p.

- pyrenaica $L$. p.

Valerianella Auricula $\boldsymbol{D C}$. a.

- brachystephana Steud. a.

- carinata Loisl. a.

- coronata DC. $\beta$. discoidea DC. a.

- dentati DC. a.

- echinata DC. a.

- eriocarpi Desv. a.

- hamata Bust. a.

- hirsutissima L.k. a.

- Morisoni $D C$. a.

- oxyrhyncha Fisch.; Mey. it.

- plagiostephana Fisch., liey.a.

- pumila DC. a.

- Szovitsiana Fisch., Mey. it.

- turgida DC. a.

- vesicaria Mönch. a.

Venidium speciosum $\boldsymbol{R} g l$, a.

Verbena hispida $\boldsymbol{R}, \boldsymbol{P}$. a.

Veronica acinifolia $\boldsymbol{L}$, a.

- agrestis $\boldsymbol{L}$. a.

- anagalloides Guss. a.

- arvensis $\boldsymbol{L}$. a.

- biloba $\boldsymbol{L}$. a.

- Buxbaumii Ten. a.

- ceratocarpa C. A. Mey. b.

- Cymbalaria Bertol. a.

- fruticulosa $\boldsymbol{L}$. p.

- longifolia Schrad. $\beta$. maritimat Koch. p.

- opaca Fries. a.

- peregrina L. a.

- polita rries. a.

- syriaca R., S. a.

- urticaefolia Jacq. p. 
Vesicaria gracilis llowk. a. - grandiftora Ilook. it.

Vibúrnum Lantana L. I.

- Tinus L. I. fr.

Vicia amoena Fisoh. p.

Vincetoxicum medium Decsne. p.

Viola elatior Fr. p.

- lutea Sm. $\gamma$. multicaulis Koch. $p$.

Vitex Agnus castus L. I. fi.

Wahlbergella aftinis C. A. Mey. p.

Wahlenbergia capensis $A$. DC: a.

- gracilis $A . D C$. a.
Wahlenbergia gracilis A. DC. $\beta$. stricta A. DC, it.

- lobefioides A:DC. a.

- nudicaulis A. DC. a.

Withania somnifera bun, a. flexuosa Dun. l. c.

Xanthium macrocarptim DC. a.

- spinosum $\boldsymbol{L}$. a.

Xanthogalum purpurascens Lall.p.

Zacyntha verrucosi Gaerln. a.

Ziziphora capitata $\boldsymbol{L}$. a.

- tenuior L. a.

\section{F I L I C ES.}

Acrophorus fallax $\boldsymbol{K y} l$. c.

Adiantum aethiopicum $\boldsymbol{L}$. fr.

- Capillus Veneris $\boldsymbol{L}$. fr.

- cuneatum Langsd, Fisch. fir.

- formosum R. Br.fr.

- Lispidulum $\boldsymbol{S} w$. fr.

- trapeziforme $\boldsymbol{L}$. var. pentadactylon Langsd., Fisch. c,

Allantodia australis $\boldsymbol{R} . \boldsymbol{B r}$. c.

- umbrosa R. Br. c.

Alsophila Fischeriana Rgl. I. c.

- Humboldtii $\mathbf{K l}$. l. c.

- radens Hook. I. c.

- subaculeatit Splitg. 1. co

- villosa Kze. 1 c.

Aneimia Pliyllitidis Sw.'c.

- $\quad$ - $\quad$ - . longi-

Aspidium caripense Mett. c.

folia Langsd,, Fisch.c.

- chrysolobum $\boldsymbol{L}$. c.

- decursive-pinnatụn $\boldsymbol{K z}$. fr.

- lobatum Prsl. d.

- macrophyllum Sw. c.

- molle Sw. c.

- obseurum Rgl. c.

- patens $S w$. c.

- Sieboldii Mett. fr.
Aspidium spinulosum $\boldsymbol{S} w$. d.

- trifoliatum $S w$, c.

- uliginosum Kze. c.

- violascens $\boldsymbol{L} . \boldsymbol{k}$. c.

Asplenium ebenum $\boldsymbol{S} w$, c.

- Nidus $L$. c.

- obtusifolium $\boldsymbol{L}_{\text {: }}$ c.

Balantium antarcticum Prsl. 1. fr.

Blechnum australe $\boldsymbol{L}$. fr.

- brasiliense Desv. 1. c.

- gracile Kaulf. c.

- Lanceola Sio. c.

- occidentale $\boldsymbol{L}$. fr.

Botrychium virginicum $S w$. p.

Chrysodium flagelliferum Mett. c.

Cibotium Schiedei Cham., Schlchtd. l. c.

Cystopteris tenuis Schott. fr. d.

Dicksonia tenera Prsl. c.

Didymochlaena lunulata Desv. l. c.

Diplazium Lasiopteris Kze. c.

- Shepherdi $L k$. c.

Doodia caudata $R$. Br. fr.

- lunulata $\boldsymbol{R}, \boldsymbol{B r}$. fr.

Hemitelia grandifolia Spr. 1. c. Hypolepis dicksonioides Hook, c. Lophosoria pruinata Prsl. I. e. Marattia Kaulfusii Kze. c. 
Nephrolepis exaltata Schoti. c.

- pectinata Schott. c.

Pheropteris effusa $\mathbf{S w}$. c.

- prolifera Mett. c.

- refracta $\mathrm{Rgl}$. c.

- tetragona Mett. c.

Polypodium areolatum $\boldsymbol{H}, \boldsymbol{B}, \boldsymbol{K}$. c. - decurrens Raddi. c.

- fraxinifolium Jacq. c.

- latipes Langsd., Fisch. c.

- morbillosum Prsl. c.

- nereifolium $\boldsymbol{S} \boldsymbol{w}$. c.

- vulgare $\boldsymbol{L}$. p.

Pleris arguta Vahl. fr:

- caudata $L$. c.
Pteris crenati $S_{1}$. fr.

- denticulata $S$ w. c.

- hastata Sw. fr.

- japonica Mett. fr.

- pedata L. c.

- sagittifolia Raddi. c.

- stenophylla Hook., Grev. fr.

- tremula R. Br. c.

- umbrosa R. Br. c.

Scolopendrium officinarum $S w$. p. angustifolium $\bar{S} w . \quad$ p.

Struthiopteris germanica Willd. p.

Woodwardia radicans sio, fr. 


\section{'S E'M I N A}

\section{IN \\ REGIONE WOLGENSI PROPE SAREPTA LECTA.}

Acroptilon Picris C. A. Mey. Alhagi camelorum Fisch, Asperula humifusa Bess. Astragalus albicaulis $D C$,

- asper Jacq.

- longitlorus Pall.

- macropus Bge.

- physodes $L$.

- virgatus Pall.

Atriplex acuminati $W, \boldsymbol{K}$. Bunium luteum $I I s / / \mathrm{m}$.

Calophaca wolgarica Fisch. Centaurea arenaria $\mathbf{M B}$.

- glastifolia $\boldsymbol{L}$.

- ruthenica Lam.

Chaerophyllum Prescottii $\boldsymbol{D C}$.

Corispermum filifolium $\boldsymbol{C}$. A. Mey.

- Marschallii Stev.

- nitidun Kit.

Crambe aspera $\boldsymbol{M B}$.

Crataegus ambigua.

Cirsium serrulatum . $\boldsymbol{H B}$.'

- setigerum Ledeb.

Delphinium puniceum Pall.

Dodartia orientalis $\boldsymbol{L}$.

Echinops Ritro $L$. var.

Eragrostis suaveolens $\boldsymbol{M B}$.

Eriosynaphe longifolia $D C$.

Euphorbia astrachuica C. A. Mey.

Ferula caspica $\boldsymbol{M B}$.

Galium tataricum Trev.

Glycyrrhiza glandulifera $\boldsymbol{W}$., $\boldsymbol{K}$.

Gypsophila paniculata $\boldsymbol{L}_{\mathbf{l}}$.

- trichotoma Wender.

Halimocnemis crassifolia C.A.Mey.

- glauca C. A. Mey.

- monandra C. A. Mey.

- Volvox C. A. Mey.

Halogeton monandrus C. A. Mey.
Iris aequiloba Ledeb.

- tenuifolia Pall.

Jurinea Eversmanni bjge.

- polyclonos DC.

Linaria odora Chav.

Linosyris villosa $D C$.

Melilotus ruthenica $\boldsymbol{M B}$.

Microphysat rubioides C. A. Mey.

Nitraria Schoberi $\boldsymbol{L}$.

Ononis intermedia C. A. Mey.

Palimbia salsa Bess.

Peucedanum latifoliun $\boldsymbol{D C}$.

- sibiricum Clans.

Rindera tetraspis $\boldsymbol{P}^{\prime} a l l$,

Rumia leiogona $C$. A. Bey.

Salsola brachiata Pall.

- laricina Pall.

- mutica C. A. Méy.

- Soda $L$.

Sanssurea crassifolia $\boldsymbol{D C}$.

Scabiosa ucranica $\boldsymbol{L}$.

Schoberia maritima $C$. A. Mey.

Scorzonera caricifolia Pall.

Serratula Gmelini Ledeb.

- xeranthemoiles $\boldsymbol{M} \boldsymbol{B}$.

Silaus Besseri $\boldsymbol{D C}$.

Statice caspia Willd.

- incana $\boldsymbol{L}$.

- sareptana A.Becker.

- suffruticosa $\boldsymbol{L}$.

- tatarica $\boldsymbol{L}$.

- tomentella Boişs.

Syrenia Lamarckiana ${ }_{i}$ ndr.

'Thalictrum commutatum $C$. A. Ney.

Tournefortia Arguzia R. $S$.

Tulipa biflora $\boldsymbol{L}$.

- tricolor Ledeb.

Vicia picta Fisch., Mey. 


\section{S E M I N A}

\section{IN PROVINCIA PERM A CLAR. KARPINSKYO LECTA.}

Aithaea ficifolia Cav.

- of ficinalis $\boldsymbol{L}$.

A marantus retroflexus $\boldsymbol{L}$.

Asperula eynanchica $\boldsymbol{L}$.

Astragalus glyeyphyllos $\boldsymbol{L}$.

- sulcatus $\boldsymbol{L}$.

Blitum virgatum $\boldsymbol{L}$.

Bryonia alba $\boldsymbol{L}$.

Bupleurum rotundifolium $\boldsymbol{L}$.

Calamintha Acinos Clairv.

Centaurea glastifolia $\boldsymbol{L}$.

- trichocephala $\boldsymbol{M B}$.

- trinervia Stephan.

Cephalaria centauroides Coult.

Colutea arborescens $\boldsymbol{L}$. .

Conringia orientalis Andrz.

Coronilla varia $\boldsymbol{L}$.

Crataegus A zarolus $\boldsymbol{I}$.

Cynoglosstun officiuale $\boldsymbol{L}$.

Datura Stramonium $\boldsymbol{L}$.

Daurus Carota $\boldsymbol{L}$.

Dianthus Pseudoarmeria MB.

Dracocephalum thymiflorum $\boldsymbol{L}$.

Eehinochloa crus galli Beauv.

Elaeagnus angustifolia $\boldsymbol{L}$.

Eryugium campestre $\boldsymbol{L}$.

- planum $\boldsymbol{L}$.

Falcaria Rivini Host.

Gentiana cruciata L.?

Glycyrrhiza echinata $\boldsymbol{L}$.

- glandulifera $\boldsymbol{W} ., \boldsymbol{K}$. (G. leguminibus asperis Pall.)

- $\quad$ - G. leguminibus laevibus Pall.)

Gypsophila paniculata $\boldsymbol{L}$.

Hyssopus ofticinalis $\boldsymbol{L}$.

Iris halophila Pall.

Jurinea stoechadifolia $\boldsymbol{D C}$.

Kochia prostrata Schrad.
Kochia scoparia Schrad.

Ligustrum vulgare $\boldsymbol{L}$.

Linosyris villosa $\boldsymbol{D C}$.

Marrubium peregrinum $\boldsymbol{L}$.

Medicago falcata $\boldsymbol{L}$.

Melica ciliata $\boldsymbol{L}$.

Moluccella laevis Georgi.

Nepeta ucranica $\boldsymbol{L}$.

Nicandra physaloides Gaerin.

Nonnea pulla $D C$.

Oenothera biennis $\boldsymbol{L}$.

Onobrychis gracilis Bess.

Ononis hircina Jacq.?

Onopordon Acanthium $\boldsymbol{L}$.

Oxytropis pilosa $\boldsymbol{D C}$.

Pimpinella 'Tragium Vill.

Phlomis pungens Willd.

- tuberosa $\boldsymbol{L}$.

Ptelea trifoliata $\boldsymbol{L}$.

Rhamuus catharticus $\boldsymbol{L}$.

- Frangula $\boldsymbol{L}$.

Rhus Typhina $\boldsymbol{L}$.

Sambucus nigra $\boldsymbol{L}$.

Saponaria officinalis $\boldsymbol{L}$.

Setaria verticillata Beauv.

sida Abutilon $\boldsymbol{L}$.

Sideritis montana $\boldsymbol{L}$.

Silene sp.

Sisymbrium pannonicum Jacq.

Stachys recta $L$.

Stipa capillata $\boldsymbol{L}$.

Teucrium Polium $\boldsymbol{L}$.

Trifolinm spumosum $\boldsymbol{L}$.

Triticum cristatum Schreb.

Verbascum phoeniceum $\boldsymbol{L}$.

Xanthium spinosum $\boldsymbol{L}$.

- Strumarium $\boldsymbol{L}$.

Zygophyllum Fabago $\boldsymbol{L}$. 


\section{ANNOTATIONES BOTANICAE.}

\section{Scolopendrlum rumieifollum Bgl.}

Frondibus longe petiolatis; petiolo juniore paleaceo, vetusto glabro; lamina e basi cordata lanceolati, apice obtusa v. subacuta, margine repanda.

Petiolus $4-6$ pollices longus. Lamina $1 \frac{1}{2} 2-3$ pollices lata et $4-6$ pollices longa. Patria Brasilia.

Olim in horto Petropolitano eultum. (Rgl.)

Asplalum Grayanum osl. ind. sem. horti Petrop. 1855 pag. 16, est A. spinulosum $\mathrm{Sw}$. $\beta$. dilatatum Koch. (Rgl.)

Aneimia repens arddi. (FI. Brasiliensis tab. I b.) Var. major mgl.

Planta ex Brasilia allata, differt folii triplo majoribus ab icone citata. Foliola variabilia, v, ut in icone citata pinnatifido - incisa; v. sublobata, v. subintegra et margine eroso - dentata. (Rgl.)'

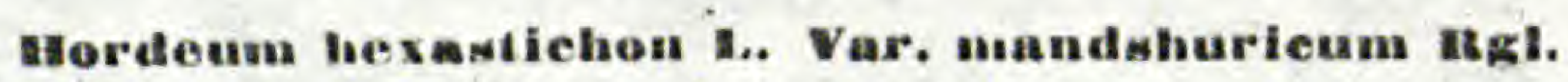

Spicis elongatis, $4-5$ poll.hlongis, cylindricis (nec cylindrico-pyramidatis ut genuinae formae). laxioribus. Colitur in Mandshuria. Semina legit Maximowicz. (Rgl.)

\section{Cupresas learwinakyana Bal.}

Ramis laxis patentibus; ramulis strictis tetragonis, abbreviatis; foliis arcte quallifariam imbricatis, late ovatis, obtysiusculis v. ramorum subacutis, lorso glandula nulla; strobili globosi squamįs breviter mueronatis, plicato - rugosis. -

Exemplaria fructifera legit Karwinsky sub $\mathscr{N} 1023$ in Mexico. Semina recepimus ex Cali ornix. -

Arbor mediocris coma expansa. Rami patentes laxi, cortice brunneo; ramuli plus minus conferti, abbreviati. Strobili globosi $3 / 4-7 / 8$ poll. in diametro, squamis peltatis, sub apice breviter umbonato-mucronatis, radiato plicato-rugosis,

C. Lindleyi Kl, cui affinis 'ignoscitur: foliis ovatis acutis carinatis dorsi glandula impressa et strobili squamis laevibus, (Rgl.) 
Tradencantia erecta Jacq, Ic, 2, t. 2354.

Cl. Kunth in Fin. pl, 3, 98 injuste ad hanc speciem ducit Cavanillesii iconem (Cav. Ic. tom. 1, 53, tab. 74) sub eodem nomine promulgatam, quae ad Tr. undatam Willd. pertinet. Differt enim haec species a Tr. erecta Jacq. praeter corollam roseam (nec coeruleam) pedunculo praeter pilos breves densos pilis multo longioribus hyalinis in sicco irregulariter perturbatis hirsuto (nec pilis brevibus tantum densis tenuibus non hyalinis pubescente). Id enim docet non solum icon Cavanillesii ipsa, sed etiam specimen a cl. Ortega olim cum Pallasio communicatum nunc in Herb. Hort. Petrop. conservatum. Praterea stamina nostrae speciei a cl. Kunthio l. c. male describuntur. Filamenta enim coerulea (nec fuscescentia nec albido-barbata), staminum trium inferiorum sursum arcuata, tuorum lateralium supra (sc. in latere interiore) a medio fere ad basim usque pilis longis coeruleis articulatis barbata, tertii medii paullo brevius glabrum; staminum trium superiorum breviorum minus sursum areuata, duorum lateralium in medio (in latere exteriore) pilis coeruleis et flavis fasciculatis barbata, tertii medii paullo brevius in medio pilis flavis (inferioribus basi subcoerulescentibus) barbatum. Antherae staminum trium longiorum oblongae, coeruleae; staminum minorum breviores sed latiores, ovales, basi apiceque emarginatae pulchre flavae. (Kcke.)

\section{Tradescantla iridescens uindl. var. slabrescens.}

Haec varietas vel potius forma et a diagnosi Lindleyana (Bot. Reg. 26, tab. 34) et a descriptione Schlechtendaliana (Hort. Hal. fasc. 3, 21, tab. 11) differt foliis subtus glaberrimis vel hinc inde pilo rarissima, conspersis, margine tantum molliter denseque ciliatis. Caulis interne glaber, superue pilis patentibus mollibus pubescens. (Keke.)

Merendera Bulboeodium Esmond. Hull. Phllom. n, $47, \mathrm{t}, 12$, f. 2 .

(Colchicum bulbocodioides Brot.)

Figura et descriptio Broteroi Phyt. Lus. sel. p. 119, t. 50, optime congruunt cum planta nostra (albiflora), quae a speciminibus quoque numerosis M. Bulbocodii genuinae in herb. Fischeriano asservatis non differt. M. Bulbocodium Redouté. Lil. t. 25 recedit staminibus acutissimis. Cl. Boiss. (Voy. bot. dans le midi de l'Esp. 2, p. 622.) Broteroi plantam cum signo? ad Merenderam filifoliam Camb. ducit, quae nobis tamen e descriptione toto coelo diversa videtur. (Rch.)

\section{Alliam praeselssum $\mathrm{Rehb.}$}

Species bona nobis videtur corollis cylindricis elongatis quamillae A. paniculati $\mathrm{L}$. et oleracei L. conspicue longioribus post anthesin etiam conniventibus nec apice ampliatis, staminibus alternis plerumque brevissimis distincta. - Nostra optime convenit cum ic. Rchb. et vix differt nisi interstitiis exacte rotundatis, quae in ic. cit. denticulis ad tilamentorum basin minutis interjecta sunt. 
A. praescissum Rehb. 1c. pl. crit. 5, p. 17, t. 429 . Ic, fl. germ. 10, t. 489 , f. 1066 . Ledeb. Fl. ross. 4 , p. 189 . A. paniculatum, $\alpha$. legitimum Ledeb. 1. c. p. 176 et herb. pro parte.

Bulbos misit Becker e regione Sareptana. (Reh.)

\section{Dianella Inconspicua $\mathrm{Kgl}$.}

\section{D. atrata Hort. Petrop.}

Glabra; foliis lineani-lauceolatis, laxe recurvatis, carinatis, planiusculis, margine et in carinae apice spinulosis; paniculae ramulis bi-trichotomis, arcuatis; pedicellis recurvis $2-4$-plo longioribus, sepalis internis trinerviis.

Folia basalia disticha, basi equitantia, superne planiuscula; usque 13 poll. longa, et $1 / 3-1 / 2$ podl. lata, coriacea, nervoso-striatil, spinulis marginalibus brevissimis erectis remotis atris. Scapus folia vix superans, subaphyllus, paniculatuș. Panicula folia subatequans vel superatus, ramis basi bractea lineari margine scabra fultis, cymosis; ramulis bitrichotomis. Pedicelli filiformes, bracteola parvula subulati fulti, $1 / 3-3 / 4$ poll. longi, recurvati. Flores parvi. Sepala exteriora oblongolanceolata, pallide fusco:rubra, 5-nervia, circiter $1-1 \%$ lin. longat; sepala interiora minora et angustiora, pallide flava, primo obscuredeinde evidenter 3-nervia. Bacca ovata, acuta, obscure olivacea. Affinis Dianellae angustifoliae Schult.

Patria ignota. (Rgl.)

\section{asubergia Meyendorthi. Mgl.}

Petalis basi' nudis, scapo submullo.

Planta speciosa caule abbreviato, $1-2$ pollicari, dense folioso. Folia nitida, glaberrima, patentia, ligulata, spinuloso-serrata, acuta v. ex apice obtuso acuminata, basi latiore caulem amplectentia et margine inda, $1 \frac{1}{2}-2$ ped, longa, $1-1 \frac{1}{4}$ poll. lata; folia superiora (floralaa) minora et omnino sanguinea $v$. batsi apiceque sanguinea et medio viridia. Thyrsus capitato-spiciformis sessilis immersus, bracteatus: bracteis linguiformibus, concavis, membranaceis, apice cucullato-obtusis, albis et apicem versus virescentibus, $1 \frac{1}{2}$ poll. longis, calycem aequantibus. Flores breviter pedicellati. Calyx superus, profunde tripartitus, albidus et apicem vers,us viridis, laciniis se invicem tegentibus, ligulatis, erectis, rotundato-acutis, pollicem longis. Corolla $1_{2}^{1}{ }_{2}$ poll. longa, calycem superans, petalis subcarnosis, unguiculatis, erectis, acutis, unguibus albis, calycem aequantibus, 'basi nudis, limbo caeruleo. Stamina tubum aequantia; antheris oblongo-lanceolatis, dorso affixis. Germen inferum, stylo staminum longitudine, stigmatibus tribus spiraliter tortis. Patria Brasilia. Affinis B. cruentae Bot. Mag. tab. 2892. Habitus omnino Nidularii. - Bilb. cruenta l. c. differt: foliis latioribus, 3 pollices latis, transverse zonato-pruinosis, involucrantibus apice tantum sanguineis; bracteis ovatis herbaceis, inferioribus calyce longioribus, petalis basi interiore squamiferis, - Nidularium splendens H. Hambrg. Bromelia Carólinae Ortg. Gartenflora tab. 211,

Bromelia Carolinae Beer,(Fam, der Brom. pag. 29), dlfferre videtur foliis $1 \frac{1}{2}$ ped. longis, $2 \frac{1}{2}$ poll. latis, floribus $1 / 2$ poll. longis. (Rgl.) 
Nidularium s.em. Jard, Heur. tom IV. Misc. pag. 60.

\section{s. scherenetiewil mal.}

Glabrum, caule humili dense folioso, foliis patentibus, apice recurvis, elongatis, lineari-lanceolatis, attenuato-acutissimis, glabris, spinuloso-serrulatis, basi Iatiore integerrima caulem amplectentibus: foliis floralibus multo brevioribus, coloratis; calycibus viridibus, trigonis, ad ovarium usque trifidis; laciniis rigidis, erectis, convoluto-appressis, ueutis. -

Caulis $1 / 2-3 / 4$ pedem altus. Folia inferiora laete viridia, sub lente impresso-punctata, $1 \frac{1}{3}$ ped. longa, pollicem lata. Flores corymbosi, subsessiles, in apice caulis axillares et terminales, corymbis lateralibus 4 floris. Folia floralia flores multo superantia et foliis inferioribus multa breviora $2-5$ poll. longa, e basi latiore integerrima elongato lanceolata, totidem purpurea v, apice tantum viridia, v. viridi-variegata. Calyx trigonus; parte inferiore tubulosa germini totidem adnata; parte superiore erecta, ad ovarium usque tripartita, pollicem v. $1 \frac{1}{2}$ poll. longa; basi bractea unica v. rarius duabus oppositis involucratus.

Corolla supera, gamopetala, calycem multo superans, $13 / 4$ poll. longa; limbo erecto, tritido, caeruleo; tubo albo; laciniis apice cucullato-concavis, obtusis, Filamenta 6, corollae tubo ad limbum usque adnata, 3 limbi laciniis opposita, 3 is alterna; antherae lineares, dorso affixae, basi breviter sagittatae. Stigma conicum, capitatum. - Patria Brasilia?

Caraguata serrata H. Petrop. et Rgl. Ind. sem. hort. Petrop. anno 56, pag. 17. (Rgl.)

vidularium purpureum seer. Die Familie der Bromeliaceen pag. 78 .

Caule aumili dense folioso; foliis erecto patentibus, apice recurvis, lineari-lanceolatis, attenuato-acutis, obscure purpureis squamis albidis lepidotis vestitis, spinuloso-serrulatis, basi latiore integerrima amplexicaulibus, foliis floralibus multo brevioribus, concoloribus; calycibus trigonis, limbo erecto, ad ovarium usque tripartito, rubro, lobis ovatolanceolatis, acutissimis, convoluto-appressis.

Folia usque $1 \frac{1}{2}$ pedes longa, $1 \frac{1}{2}$ poll. lata. Corolla superne carminea. Cetera ut praecedentis. Planta, quam cl. Beer descripsit, differt foliis nitidis glabris, serrulatis (nec mucronato-serrulatis).

Pitcairuia discolor H. Petrop. (Rgl.)

\section{Pitcairuia nammea Lindl.}

Var $\alpha$. genuina foliis subtus lanuginosis.

Pitc. flammea Lindl. Bot. Reg. tab 1092.

Pitc. Olfersi Lk. in Verh. d. Gartenbauvereins f. d. Preuss. St. tom. 7. 353, tab, 3 (Icon non differt nisi pedunculis florumque basi viridibus nec coccineis).

Pitc, latifolia Hort. nonnull.

Var. $\beta$. glabra Rgl. et Kicke. foliis utrinque glabris.

Pitc. splenden, Hort. (Vilain) nec Warsczew. Berliner Allg. Gartzt. 19 (1851) 176. (Kicke.) 


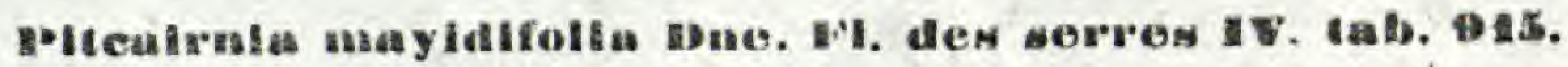

Petalis basi squauniferis. Puya Funkiana et mayidifolia Hort. Puya mayidifolia Morr. Ann. V, tab. 289 a planta nostra diversa. Pitcairnia Funkiana Rgl. Grtfl. tab 109 ad P. macrocalycem Hook. Bot. Mag. tah. 4705 pertinet. (Rgl.)

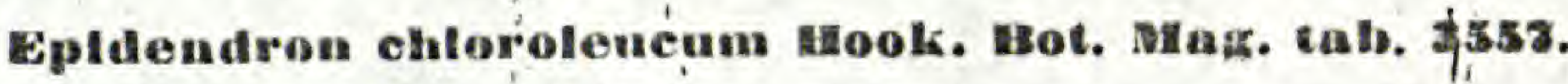

\section{Var. Iusco-luteuni Rgl.}

Foliis angustioribus(lineari-ligulatis), sepalis petalisque fusco-luteis, labelli lobo intermedio breviter unguiculato venis ramosis rubris elevatis percurso. Cetera ut in Hookeri descriptione et icone nec ut in Lindleyii Fol. orchilaceis 1237 . Accepimus e Mexico. (Rgl.)

\section{Maxillarla porrecta Gladl. Var. Iutea. Eght.}

Pseudobulbis oblongis, compressis, plus minus costatis, '2 -3 poll. longis, $1 / 2-2 / 3$ poll. latis; folies binis, lineari-lanceolatis, acutis; sepalis lanceolato-falcatis, incurvo-patentibus, luteis, margine et in carina dorsi rubentibus; petalis lineari-lanceolatis, paullo minoribus, luteis; labello oblongo, concavo, trilobo, luteo, atropurpureo-narginato, lobis lateralibus rotundatis, lobo intermedio margine crispulo et apice obtuso recurvo. - Galeotti misit plantas vivas e Mexico. An spec. nova? ( $\mathrm{Rgl}$.)

Gneidlum armillare Lind. (Folia orchidacea $\mathscr{N} \cdot 182,12$. Verruci tuberculati.)

Pseudobulbis compresso-ovatis, apicem versus, attenuatis, sulcatis, 2 -3-phyllis; foliis oblongo-ligulatis, apice obtusis, inaequaliter bilobis; panicula gracili, dependente, ramosa, multiflora; bracteis minutis, ovatis, apiculatis; sepalis patentibus, margine leviter undulatis: 3 exterioribus oblongis, apiculatis: 2 interioribus lanceolato-falcatis acutis, labello trilobo pandurato: lobis basilaribus rotundatis porrectis: lobo intermedio lateralibus paullo latiore, late-obcordato: callo plurituberculato, e carinis tribus flexuosis apice in dentem validum exeuntibus, verruculis circumstantibus composito; columna apice alata: alis basi truncatis, apice acuminatis, margine inaequaliter denticulatis.

Pseudobulbi 3 poll. et ultra longi, $1 \frac{1}{4}$ poll. lati. Folia usque $1 \frac{1}{4}$ ped. longa, $3 / 4-1^{1 / 2}$ poll. lata. Paniculae $3-4$ pedes longae rami laxi racemosi, simplices v. ramosi. Flores pallide citrini, in sepalorum basi et labelli centro fusco-maculati v. fasciati.

E Venezuela misit Wagener. An satis diversum ab 0 . aurifero Rehb, fil. Linn. XXII. pag. 847? O. auriferum Lindl. Fol. orchid. . 181 nobis ab 0 . aurifero Rehb. fil. 1. c. sepalorum forma diversum videtur et probabiliter forma eadem $\mathrm{cum}$ subsequente. (Rgl.) 


\section{0 - \\ Oneldium armillare lindl. $\beta$, stemonepalum Egl.}

Sepalis petalisque falcato lineari-lanceolatis, panicula adscendente. Lausberg e Columbia misit. Rgl.)

\section{Maranta $\mathbf{L}$.}

\section{Subgen. Saranthe Ifgl, et Ecke.}

Inflorescentia spiciformis, cristata, densa; bracteae oppositae dense imbricatae, secundae (ad alterum latus conversae ibique flores foventes); staminodia duo externa satis parva, non labelliformia; calyx in fructu persistens bracteis communibus inclusus; semina arillo instructa. - Folia marginibus parallelis, ex apice rotundato-obtuso vel obtusiusculo breviter acuminata. Rami florales apice spicam unam vel duas vel plures paniculatas gerentes. Bracteae distichae sed in unum latus admodum spicularum Cynosuri cristati L. conversae, et flores 2 vel plures qui in unum latus spectant foventes. Flores parvae vix e bracteis eminentes, bracteolati. Petala et staminodia basi infra medium connatae superne convolutae in tubum breviusculum latiusculum; illa appressa, aequalia; staminodia limbo parvo subarrecto. Staminodia duo externa subaequalia breviuscula, tertium medium intus callo crasso ornatum, quartum intimum cucullatum stigma obtegens. Hoc subgenus spicis confertis, bracteis imbricatis distichis sed ad modum Cynosuri cristati unilateraliter conversis, floribus vix eminentibus, tubo brevi latiusculo staminodiis duobus externis subparvis insigne complectitur quinque species habitu etiam affines, quarum tres per hortos longe lateque divulgatae sunt.

Primo intuitu generi Calatheae quidem haud absimile sed differt tubo corollae brevi quam germine crassiore (nec tenui gracili elongato), staminodiis externis duobus (nec uno), anthera libera (nec a medio ad busim cum appendice filamenti parva petaloidea connata). Praeterea accedit, quod (monstrante specimine Riedeliano M. Luschnathianae spontaneo et aliis cultis) specimina uberius enata in apice rami florifeni praeter spicas proferunt folia et ramos iterum spicigeros, atque folia accuratius inspecta et forma et colore et splendore magis ad Marautam arundinaceam $L$. et species affines accedunt. - Nomen e vocibus

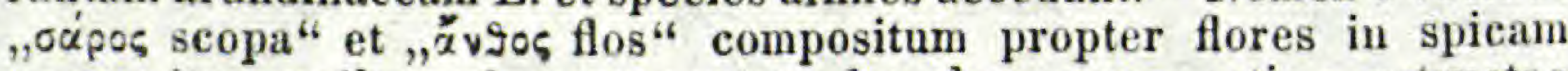
compositam unilateralem congestos ad modum scopae setis constructae (Germanis: Handfeger).

\section{1) ssaranta (Saranthe) wetosa A. Btetr.}

Phrynium setosum Rosc. Scit. tab. 41.

Maranta secunda Hort. Edinb.

Foliorum vaginis et spicarum pedunculis et bracteis chartaceis viridibus persistentibus lanceolatis longe acuminatis inferioribus ubique reliquis margine et a medio ad apicem setosis; spicis geminis vel pluribus aggregatis.

Icon Roscoeana quoad plantam totam optina! Pili setosi flavescentes praesertim ad vaginarum et bractearum margines densi et longi. 
Folia parallelo-oblonga ex apice obtuso vel obtusiusculo subito breviter acuminati, lamina usque 16 pollices longa et usque 4 poll. et $7 \frac{1}{2}$ lineas lata. Ramus Horifer setoso-hirsutus. Spica (si tres) infima pedicellata, pedicello $3 / 4-1$ pollicari; media sessilis et basi bractea reliquis longiore suffulta; summä breviter pedicellata; $1 \frac{1 / 2}{2}-2$ pollices longae, $1 \frac{1}{4}$ pollicem latae. Bracteite erecto-patentes, circiter pollicares. Bracteolae communes binos flores includentes late ovatae, acutiusculae, dorso bialatae, alis pilosis purpurascentibus, ceterum glabrae, membranaceae, hyalino-alibi lae. Bracteolae speciales minores ovales, acutiusculae et dorso uni-alatae vel obtusae et exalatae, glabrae, membranaceae, hyalino-ilbilae. Germen obovato-cylindricum, pilis appressis longis vestitum, flavidulum. Bepala 3 libera, corollam dongitudine aequantia, acutiuscula, apice cucullata, pilis longis arrectis praesertim versus apicen vestita, concava, membranacea, pellucida, albida margine purpurascentia. Petala cum staminodiis infra mediuin ad basim usque in tubum calyce plus dimidio breviorem germine latiorem connata, ovalia, obtusa, arrecto-pilosa, navicularia, mémbrinacea, hyalino-albida, staminodiis appressa, marginibus se non tegentibus. Staminodia superne in tubum vix limbatum convoluta; duo exterua aequalia, spathulato-obovata, rotunlato-obtusa, albilit. Staminotia interna cum stamine fertili 3 ; extimum callosum duobus externis vix brevius sed paullo latius, late ovatum obtusissimum callo magno in medium floris porrigente saturate flavo instructum pt praeterea in loco alio callose incrassatuin, margine albidum medio flavun; intimum inaequilaterum, spathulatum, fere unguiculatum ad alterum latus auricula parum deflexa instructum, flavidum, stylum cucullato-obtegens. Stamen fertile: Anthera libera; appendice petaloidea illam asquante, oblonga, albida apice flavida, filamento usque ad basim antherae adnata. Stylus rectangulariter infractus, demum incurvatus, stomate truncato.

\section{2) Maranta (Saranthe) Wuschnathlana Mgl. et Wéke.}

Foliorum vaginis et bractẹ is chartaceis viriaibus persistentibus ovatis acutis margine strigilloso-setosis, spicarum pedunculis glabris; spicis plerumque geminis, raro solitariis.

Caulis perbrevis. Folia distiche cespitosa, longe pedunculata, basi vaginata. Vaginae margine flavescenti-setosae ceterum cum pedunculis pilis flavescentibus subsétosis hirtellae vel puberulae, usque peilales. Petioli compressiusculi, paullo infra laminam articulato-incrassati, snpra a vagina usque ad articulum sulco instructi, usque 20 pollicares et longiores, articulo supra'linea, hirtella iustructo vel glabro $1-2$ pollicari. Lamina parallelo-elliptica, ex apice rotundato breviter apiculata, basi rotundata breviter producta, glaberrima, nervo medio supra sulcato subtus prominente, supra obscure viridis, subtus pallidior $7-12$ pollices longa, $3 \frac{3}{4}-6 \frac{1}{2}$ pollices lata. Ramus florifer e medio progrediens, setoso et sparse pilosus demum glabrescens, basi foliis involntus, medio nudus vel folio instructus, apice folium et spicas geminas proferens, usque ald apicem spicae summae $1 \frac{1}{2}$ pedes superans. Spicae geminae, usque ad $13 \frac{4}{4}$ pollices longae $1 \frac{1}{4}$ pollice paullo latiores, summa brevius pedunculata vel sulssessilis, pedunculo glabro spicae inferioris usque $2_{2}^{1}$ pollicari vel multo breviore. Fo. 
lium sumnua basim spicarum vaginans interdum ad vaginam redactum. Bracteae ovatae acutae margine flavescenti-setosae ceterum glabrae, distichae sed in unum latus conversae ibique in axillis flores circiter 4 foventes, in altero latere steriles, invicem se amplectentes et arcte appressae, virides, circiter 1 pollicem longae, hasi $3 / 4$ pollieem latae. Flores subsessiles, omnes et singuli bracteolis involuti. Bracteolae communes lato-ovatae, leviter retusae, apiculatae, membranaceae, dorso alis duabus membranaceis rigido-pilosis ornatae, albidae. Bracteolae speciales oblongae, acutiusculae, a medio ad apicem margine rigido-pilosae, naviculares, membranaceae, hyalino-albidae. Germen inferum obovato-cylindrictim, pilis appressis strictis praesertim apice vestitum, albidum, uniloculare, uniovulatum et corpusculo placentario instructum. Sepala corollae appressa eamque fere totam involventia, bracteolis specialibus simillima. Petala infra medium ad basim usque in tubum calyce plus dimidio breviorem germine latiorem connata, oblonga, obtusa, apice cucullata, glabra, navicularia, membranacea, hyalino - albida, staminodiis appressa, marginibus se tegentibus. Staminodia superne in tubum vix limbatam convoluta; duo externa aequalia obovata, rotundato-obtusa, albida, reliquis pauilo longiora. Staminodia interna cum stamine fertili 3 , extimum callosum lato-obovatum, rotundato-obtusum, intus callo magno in medium floris porrigente saturate flavo instructum, margine albidum medio flavum; intimum inaequilaterum, spathulatum, unguiculatum, ad alterum latus auriculatum auricula lata parum deflexa, albidum, stylum cueullato-obtegens. Stamen fertile: Anthera libera; appendice petaloidea illam fere aequante oblonga albida apice flavida, filamento usque ad basim antherae adnata. Stylus apice rectangulariter infractus, stomate truncato. Fructus membranaceus unilocularis monospermus. Semen truncato-ovale, tuberculatum, arillo biligulato instructum. Marantae setosae A. Dietr. valde affinis sed optime distincta pilositate evidentius minore praesertim bractearum, foliis brevioribus et latioribus, bracteis ovatis acutis (nec lanceolatis acuminatis). - E Brasilia veris ile a cl. Riedel et Luschnath missa.

\section{Maranta (Aaranthe) Jeptestachya Hul. et Keke.}

F thorum vaginis margine inferne pubescentibus ceterum cum spicar peduncuio bracteisque albido-membranaceis glabris; spicis solitaris.

Caulis perbrevis. Folia longe petiolata, basi vaginata. Vaginae mirg ne pilis flavescentibus pubescentes ceterum glabrae usque 8 polheare 3. Pedunculi compressiusculi, apice articulato-incrassati, supra a vagina usque ad articulum vix sulcati, usque 19 pollicares, articulo 1- $1^{3}$, pollicari. Lamina oblongo-elliptica, marginibus subparallelis apice qubito breviter acuminata, basi obtusiuscula breviter producta, glaberrima, nervo medio supra sulcato subtus prominente, supra obseure viridis et nitida subtus pallidior, usque ultra pedem longa et usquie uitra 4 pollices lata. Ramus florifer inconspicuus, foliis multo brevior et occultus, glaber, apice vaginam (non folium) et spicam solitariam terminalem rarissime duas gerens, usque ad apicem spicae 9 pollicaris. Vagina peduncuIum spicae $3 \frac{1}{4}-4^{3 / 4}$ pollicarem longe invoivens, glabra. Spica $1 \frac{1}{2}-1^{3 / 4}$ pollices longa, usque $3 / 4$ pollicem 
fere lata. Bracteae ovales, obtusae, basi barbulatae ceterum glabrae, distichae sed in alterum latus conversae ibique in axillis flores binos foventes, in altero latere steriles et superiores tantum basi se amplectentes inferiores remotiusculae, tenui-membranaceae, subpellucilae, albidae, usque $1 / 2$ pollicem longae et $4 \frac{1}{2}$ lineas latae. Flores bracteis communibus duabus vel una involuti, breviter pedicellati, pedicellis basi connatis. Bracteolae late ovatae, obtusae, glabrae, membranaceat, hyalino-ilbidice, exterior laevis, interior oblique carinata; si una adest, um irterior. Germen obovato-cylindricum, appresse pilosum, flavum, uniloculare, uniovulatum, corpusculo placentario instructum. Sepala 3 oblonga, acuta, glabra, trinervia, membranacea, hyalino-albida, corolla plus duplo breviora, sese invicem marginibus non attingentia. Tubus floris brevis germine latior. Petala 3 aequalia, oblonga, obtusa, glabra, membranacea, hyalino-albida, cum staminodiis superne in tubum convoluta. Staminodia duo exterua subaequalia, obovata, rotunndato-obtusa, leviter retusa, glabra, albida. Staminodia interna cum staunine fertili 3; extimum lato-obovatum, obtusissimum leviter emarginatum, callo magno intus porrigente flavo instructum, margine albidum medio flavidum; intimum inaequilatere spathulatum, ad alterum latus auriculatum, auricula lata paullo deflexa, inferne flavum, stigma cucullato obtegens. Stamen fertile: Anthera libera; appendice oblonga usque ad basim antherae filamento adnata flavida demum albida quam anthera longiore. Stylus apice primum rectangulariter infractus demum incurvus, stomate truncato. Bracteae una cum flóribus non foecundatis decilint. Si flores foecundantur, verisimile bracteae etiam persistunt. Maruntae setosite A. Dietr. et Luschnathianae Rgl. et Kcke. bracteae, ne foecundatis quidem floribus, semper persistunt. (Kcke.)

\section{sorocea Aug. St. Hil.}

Genus Artocarpearum Soroceam a cl. St. Hilaire anno 1821 in Mem. du Mus. tom. 7, 469 pronuneiatum nuperrime autores diversissime non solum verbis interpretati sųnt sed etiam iconibus illustraverunt. Cl. Trecil enim (in Ann. d. sc. nat. ser. 3, tom. 8, pag. 145 tab. 6, fig. 182 - 188) huic generi adscribit: „Parigonium tubulosum cum ovario connatum, versus apicem liberum; stylum brevem crassum bifidum, lobis intus "stigmatosis; ovarium inferum uniloculare uniovulatum." Contra cl. Miquel (in Mart. et Fenzl. Fl. Brasil. fase, 12 pag. 112 tab. 34) contendit: „Perigonium urceolato-tubulosum ore integrum receptaculi ramificatione partim immersum vel suffultum; ovarium primo superum pedetentim cum perigonio coalescens; stylum brevissimum, stigmatibus 2 intus stigmatosis; fructum cum perigonio receptaculi parte baccante coalitum receptaculi ramulo tumefacto sustentum."

Itaque affirmat cl. Trecul ovarium inferum et perigonium superum; contra $\mathrm{cl}$. Miquel ovarium primo superum demum cum perigonio connatum atque totum fere florem femineum receptaculi prolongatione inclusum, quae quum a parte florem suffulciente articulationis specie separata sit, perigonium essé videatur.

Si denique icones Gaudichaudianas (in Vaillant Voy. d. I. Bonite Botan. tab. $71-74$ ) descriptionibus carentes contulerinats, in tab. 71 (Sorocea Hilarii Gaud.) germen inferum in tab. 74 vero (S. Guillemi- 


\section{$-34-$}

niana Gaud.) germen quidem inferum fructum vero superum, denique in tab. 72 (S. racemosa Gaud.) germen semisuperum invenimus.

Restat igitur examinatio speciminum, quae mihi in Sorocea Hilarii Gaud. et Guilleminiana Gaud., quum germina et fructus inferos demonstraverit, cl. Treculii et descriptionem et iconem prorsus comprobavit contra cl. Miquelii opinionem refutavit. Itaque ad characteres generis a cl. 'Trecul l. c. propositos nihil addo, nisi quod stamina semper inclusa sint, quae in genere affini Trophide perigonium superant.

C1. Gaudichaudii figuram 12 in tab. 74, quae in fructu germen-superum ostendit, esse minus recte delineatam, mihi specimina Riedeliana fructibus submaturis instructa persuaserunt.

Colitur jam multos per annos Sorocea quaedam a cl. Riedelio missa, nune flores masculos, olim in speciminibus alteris femineos etiam proferens, quae nobis varietas Soroceae Hilarii Gaud. videtur esse. Praeterea praesto sunt specimina exsiccata Riedeliana Soroceae alius, quam esse S. Guilleminianam existimo. Utriusque speciei descriptiones, quum adhuc desiderentur, botanophilis hic offero.

\section{Sorocea milarii Gaudich.}

Foliis oblongis vel obovato-oblongis, abrupte et longiuscule acuminatis, integerrimis vel versus apicem repando-dentatis, glabris; racemo (receptaculo) longo vel longissimo; femineo rigidulo et superne crassiore; floribus femineis laevibus; germine et fructu pilis brevibus hispidulo vel pubescente; perigonii depresso-globosi ore haud exserto, stigmatibus lanceolatis, obtusis, breviusculis.

Var. $\alpha$. genuina. Foliis magis dentatis, racemo femineo circiter 3pollicari, cum pedicellis glabro; germine pilis brevibus rigidis sparsiusculis hispido.

Sorocea Hilarii Gaudich, in Vaillant Voy. d. l. Bonite tab.

71. (Iconem tantem vidi).

Sorocea ilicifolia Miq. $\beta$. Hilarii Miq. in Mart. et Fenzl. Fl. Brasil. fasc. 12 pag. 213.

Var. $\beta$. graciliflora Rgl. et Kcke. Foliis minus dentatis vel integerrimis; racemo masculo laxo pendulo $2 \frac{1}{2}-7 \frac{1}{2}$ pollicari; femineo patenti vel arrecto, cum pedicellis et germinibus pilis brevibus rigidulis densis pubescente.

Descriptio var. $\beta$ : Arborea. Rami juniores et petioli brevissime puberuli mox glabrati. Folia alterna, brevissime petiolata, oblonga vel obovato-oblonga, abrupte et longiuscule acuminata, integerrima vel versus apicem repando-dentata, glabra, perganena, praesertim juniora supra nitida subtus nitidula et hic nervo medio et lateralibus pinnatis ante marginem arcuate conjunetis prominentibus, cum petiolo $14 / 2-3$ lineari (in speciminibus spontaneis $3-4^{1} / 2$ lineari) $23 / 4-6$ pollices longa, $2^{1} / 2-23 / 4$ pollices lata. Stipulae lineari-lanceolatae, acuminatae, puberulae, caducae. Racemus (receptaculum) bracteis oblique peltatis vix puberulis ornatus, masculus femineo paullo longior et gracilior, puberulus, usque ad basim florifer. Flores utriusque sexus breviter pedicellati; pedicello masculo paullo tenuiore et longiore, glabriusculo. Fl. masc. Perigonium tetraphyllum, glabrum, foliolis subrotundis, flavescenti-viridibus, herbaceis, ima basi connatis. Stamina 4 inclusa perigonii foliolis opposita. Filamenta brevia, cras- 
siuscula, glabra. Antherae extrorsae, breves, basifixae, connectivo crassiusculo viridi, quadrilocellatae, locellis parallelis longitudinaliter dehiscentibus flavidis. Pistilli rudimentum nullum. Fl. fem.: Germen inferum primum breviter obovatum, demum cylindraceo-ovale, laeve, uniloculare, uniovulato, ovulo ex apice parietis pendulo. Perigonium levissime puberulum, depresso-globosum, apice paullo impressum, e partibus, 4 carnosis connatum, clausum et stigmata tantum permittens. Stigmata 2 parva, recurva. Fructum maturum non vidi; simmaturus ovalis et perigonio depresso-globoso, terminatus. Sectio verticalis optime congruit cum fig. 9 et 11 tab. 71 Gaudichaudiana l, c., nec non cum fig. Treculiana 186 tab. 6 tom. 8 ser. 3 Ann. d. sc. nat. Soroceae Guillemianae exceptis tuberculis. - Differt haec species a $\mathrm{S}$. racemosa Gaudich. 1. c. racemis elongatis affini: foliis non adeo exacte et parallelo-oblongis saepe dentatis pro ratione longitudinis latioribus, racemisi femineis fere usque ad basim floriferis (nec a basi ultra medium sterilibus et nudis), fructibus pubescentibus (nec glabris). A S. Uriamem Mart. differt foliis multo majoribus; a S. muriculata Miq. et Guillemiana Gaud. fructibus laevibus (nec tuberculosis); a S. amazonica Miq. racemis longioribus, perigonio ore non membranaceo, stigmatibus obtusis, fructibus pubescentibus (nec glabris); a S. macrophylla Gaud. foliis minoribus, minus exacte oblongis, dentibus paucioribus et minus acutis. - Cl. Miquel Soroceam ilicifoliam suam infauste cum S. Hilarii Gaud. conjunxit, quapropter conferas quae ad finem Soroceae Guilleminianae disseram.

\section{sorocea Guilleminiana Gaudich. in Vaillant Voy. d. 1. Bonite tab. 74 fig. $8-16$.}

Sorocea ilicifolia Miq. in Mart. et Fenzl. Fl. Brasil, fasc. 12 , pag. 114

Sorocea ilicifolia Miq. $\propto$. grandifolia Miq. 1. c. pag. 213.

Foliis oblongis, acuminatis, praeter basim remote spinoso-serratis, glabris; racemo (receptaculo) masculo brevi, femineo longiore et superne crassiore; pedicellis femineis puberulis; germine et fructu dense tuberculato, glabre; perigonio semigloboso ore haud exserto stigmatibus persistentibus lanceolatis obtusis coronato.

Arbor 20-25 pedalis (fide cl. Riedel). Folia alterna, breviter pedicellata magna, marginibus subparallelis vel versus appicem parum divergentibus, basi apiceque acuminato excepto utrinque dentibus spinosis pungentibus $11-13$ instructa, pergameno-coriacea, glabra, supra nitida subtus nitilula et hic nervo medio et lateralibus pinnatis ante marginem plerumque rectangulariter conjunctis prominentibus, cum petiolo ( $4-6$ lineari) $5 \frac{1}{2}-8 \frac{1}{2}$ pollices longa, $1 \frac{1}{2}-2$ pollices lata. Stipulae lanceolatae, acuminatae, glabrae, caducae. Racęmus (receptaculum) bracteis peltatis margine ciliato-puberulis ornatus; masculus femineo multo brevior, petiolo circiter duplo longior, leviter puberulus, solitarius vel genini, fere usque ad basim florifer, patens vel arrectus, $6-10 \frac{1}{2}$ lineas longus. Flores masculi pedicellati, pedicello vix puberulo. Perigonium tetraphyllum; foliolis subrotundis, glabriusculis (luteo-albidis teste cl. Riedel). Stamina 4. Racemi feminei multo longiores, crassiores, patentes vel erecti, levissime puberuli, 
basi nudi, maxima parte floriferi, $2 \frac{1}{2}$ pollicares (in icone Gaudichaudiana multo breviores sed forsan aetate destructi). Flores feminei pedicellati; pedicelli crassi pilis brevissimis rigidulis puberuli. Germen inferum dense tuberculatum, glabrum. Perigonium carnosum, connatum, semigloboso-ovatum, exsiccatione? subtuberculatum. Stylis versus basim incrassatus, brevis, inclusus. Stigmata 2 majuscula, recurvata. Fructus (Putamen) tuberculis magnis rotundatis prorsus obtectus, uniovulatus, ovulo ex apice pendulo. - Planta nostra cum icone Gaudichandiana optime congruit, exceptis dentibus in icone illa non in spinam pr ductis. Diagnosis contra Miqueliana Soroceae ilicifoliae suae optime in nostram plantam cadit, si neglexeris pilos conicos petioli, quos non vidi. Folii vero et forma et dentes et nervi adeo sunt insignes, ut non dubitem, quin nostra planta eadem sit quae Miqueliana. Nescio tamen, cur cl. autor speciem suam cum Sorocea Hilarii Gaud. conjunxerit et foliorum forma et dentibus valde diversa. Sorocea Guilleminiana Gaud. fructibus tuberculatis proxime accedit ad S. muriculatam Miq., sed differt tuberculis majoribus et densioribus foliisque multi- et spinoso-dentatis (nec integerrimis vel superne repando-dentatis). A S. macrophylla Gaud. foliorum forma et dentibus saepe crebris affini differt racemis masculis brevibus ubique floriferis (nec elongatis gracilibus a basi ad medium nudis et sterilibus).

Adn. Cl. Miquel 1. c. pag. 216 et tab. 34 fig. III iconem Treculianan Soroceae Guilleminianae se depinxisse false affirmat, quum cl. Treeul in illa icone florem mascuium Soroceae racemosae Gaud. in Ann. d. sc. nat. 3 ser. 8 tom. 1507 pag. se delineasse contendit.

Soroces racemosa Gaud. in Vaill. Voy. d. 1. Bon. Tab. 72.

Foliis subparallelo-oblongis, acuminatis, integerrimis vel versus apicem parce dentatis, glabris; racemo (receptaculo) utriusque sexus longissimo gracillimo usque ad medium nudo superne florifero; floribus femineis et fructibus laevibus.

Suppetunt specimina mascula et in Brasiliae montibus Corcovado a ci. Langsdorff lecta et in horto Petropolitano culta. In icone Gaudichaudiana planta feminea delineata est. Sequitur descriptio plantae masculae. Arborea. Rami juniores petioli racemi breviter puberuli, mox abrati, Folia alterna, brevissime petiolata, pergamena, supra obscue viridia, subtus pallidiora et hic nervo medio et lateralibus pinnatis ante marginem arcuate conjunctis prominentibus, cum petiolo $5-6$ pollices longa, $13 \frac{1}{2}-21$ lineas lata. Stipulae lineari-łanceolatae, acuminatae, puberulae, caducae. Racemus (receptaculum) bracteis oblique peltatis parvis hinc inde conspersus, pendulus, a basi ad medium usque nudus, $4^{1} / 2-9$ pollicaris. Flores pedicellati, pedicello glabriusculo, obovati, basi cuneati. Perigonium tetraphyllum, foliolis ovatis obtusis basi connatis glabris. Stamina 4 , perigoniis foliolis opposita et ea aequantia. Filamenta brevia, glabra, lata. Antherae basifixae, connectivo crassiusculo, locellis flavidis. - Foliis pro ratione longitudinis angustis integerrimis vel parce dentatis, racemis longissimis inferne usque ai medium nudis insignis. A.S. Hilarii Gaud. praeterea differt floribus masculis tenuius pedicellatis obovatis basi cuneatis (nec globosis basi rotundatis). (Keke.) 


\section{$=$ \\ Banksla Gïintherl ragl.}

Frutex basi valde incrasşatus, ramulis glaberrimis v. junioribus pilosis; foliis elongato-linearibus, apice truncatis v. rotundatis, basin versus attenuatis margine serratis, serraturis mucronato-pungentibus, supra glabris nitidis, subtus niveo-tomentosis, venosis; spica cylindrica; calycibus pilosis marcescentibus; stigmate oblongo. - Eolia-superne $3 / 8$ poll. lata, $4-5$ poll. longa, ad basin usque mucronato-serrata. Flores ochroleuci. - B. cylindrostachyae Lindl. proxima, sed ramulis mox glaberrimis, foliis mucronato-serratis calycibusque pilosis bene distincta. (Rgl.)

Statice denudatu Rgl, et Keke.

Statice ser. II Corolla gamopetala; sect. IX Polyarthrion: Calyx insertione rectus, e bractea longe exsertus, tubuloso-subinfundibuliformis, limbo breviter 5 lobo. Corolla rosea ad'faucem usque gamopetala infundibuliformis, tubo e calyce exserto, post anthesin non corculata. Styli fere a basi liberi. Boissier in DC. prodr. $12,667$.

Frutescens; ramis adultis denudatis, juvenilibus foliatis; foliis oblongo-spathulatis, in petiolum longum sensim attenuatis, obtusis, utrinque lepidibus calcareis conspersis; scapis superne ramosis parce calcareo-punctatis, ramis omnibus floriferis; spiculis unifloris erectis, in spicas breves laxas paucifloras dispositis; lobis calycis subulatis; corolla purpureo-rosea.

Planta 21 pollices alta. Caulis erectus, parce ramosus, cum ramis 17 pollices altus, glaber, fuscus, $2^{1} / 2$ lineas in diametro crassus. Rami juveniles fuscae ut folia lepidibus calcareis conspersae. Folia mutica vagina clausa ramum arcte amplectentia, caesio-viridia, crassiusula, cum petiolo inferne teretiusculo 2 -fere 3 pollices longa, $3-41 / 2$ lineas lata. Scapi in ramulis terminales, inferne simplices, nudi vel bracteis 1 vel 2 instructi, superne ramosi, cum ramis neque fragilii.as neque numerosis suberectis $6-7$ pollicares. Bractea amplexicaulis et clausa, obtusiuscula, lepidibus calcare is conspersa, viridis margine fusco non membranaceo. Bracteola florem cingens quam ille duplo brevior cylindrico-involuta, obtusa, dorso subcarinata, parce calcareo-punctata, viridis, margine atropurpureo. Calycis limbus rectus albidus tenuimembranaceus quinquedentatus, dentibus nervis rufis pereursis. Corollae tubus calyce multo longior, a basi ad limbum usque $1 / 2$ pollicaris; limbus patentissimus circiter $7 \mathrm{lin}$ s latus, lobis oblongo-obovatis, obtusis, interdum leviter returis. Filamentá tubo corollae alte adnata vel certe arcte adhaerentia. Styli versus basim connati. - A Statice caesia Gir. differt statura fruticosa, floribus majoribus suturatius coloratis, foliis minus calcareo-pruinosis non radicalibus, angustioribus, ramis scapi paucioribus non fragillimis; bracteis majoribus quam bracteolae florem involventes dimidio tantum minoribus. (Kc)ke.)

Aster pyrenacus DC. Fl. fr. n. 3139. Prodr. 5. p. 229.

Praeter synonyma Decandolleana sequentia certe huc spectant: $\Lambda$. sibiricus Willd. Sp. pl. 3. p. 2042. (excl. syn. Gmel., var. $\beta$. et patr.) 
Nees. Aster. p. 37. (excl, syn. L., var. $\beta$. et patr.) Ledeb. Fl, ross. 2, p. 475. (excl. cit. ross. et habit.) et herb.

Certissime non in Sibiria! (Rch.)

Aster sthiricus L. Sp. 1326.

Species quamvis bene distincta valde polymorpha. Variat caulibus adscendentibus erectisve bipollicaribus et bipedalibus (elatis saepe flexuosis: A. flexuosus, ircutianus), nunc multifloris nune $1-4$ floris; foliis utrinque acutis vel inferioribus obtusis superioribus basi subcordata subssessilibus, utrinque scabriusculis vel subglabris; involucri squnamis linearibus, late lanceolatis plus minus foliaceis (A. espenbergensis).

Lusus memorabilis habitu insignis vix tamen varietatem constituens, quum una eademque radix genuinos caules haud raro proferat, $\mathrm{A}$. Richardsonii $\beta$. giganteus Hook. est. Hic plerumque ramosissimus, elatus, caulibus crassioribus villosis, foliis latissimis inciso-serratis fere omnibus acutis, superioribus ovato-lanceolatis, subtus plus minus tomentosis; involucri squamis exterioribus saepe maximis foliaceis.

Hujus speciei synonyma sunt: A. sibiricus DC.Prodr.5. p. 231. (excl. syn. Fisch.) Turcz. Fl. baic. - dah. Addenda p. 33, A. foliis ovatis, oblongis, supra serratis, caulibus striatis, pedunculis unifloris, umbellatis Gmel. Fl. sib. 3. p. 186. n. 152. A. montanus Richards. in Frankl. Journ. to the Polar Sea App. p. 760, (non Nutt.) Torr. et Gr. Fl. of North Am. 2, p. 107. A. Richardsonii Spr. Syst. 3, p. 528. Nees. Aster. p. 30. Hook. Fl. bor. - am. 2. p. 7. Torr. et Gr, 1. c. Ledeb. Fl, ross. 2. p. 475. DC.1. c., p. 229 . Turcz. 1. c., vol. 2, fasc. 1, p.11. Rgl. et Til. Fl. ajan. n. 161. A. ircutianus DC. 1. c. (excl. syn. Turcz.) A. flexuosus Fisch. in Mém. de la soc. des nat. de Mosc. 3. p. 73, et herb. (von Nutt.) A. Fischerianus DC. 1. c. p. 24 . Ledeb. 1. c. p. 474 . Turcz. 1. c. A. espenbergensis Nees 1. c. p. 36 .

Ad lusum supra memoratum spectant: A. Richardsonii $\beta$. giganteus Torn et Gr. 1. c. A. foliis ovatis etc. Gmel. 1, c. t. 30, f. 1.

Vidi specimina e Sibiria altaica (Ledebour, Gebler, Politow, Mardofkin), baicalensi prope pagum Wedenskaja (Turcz.), orientali prope Ochotzk (e specim. cult.), inter Wilnisk et Olekminsk (Kruhse), ad fluv. Ischiga (Kruhse), prope Ajan (Tiling), arctica pr. Kolimsk (Scharypow); hamtschatka(Rieder, Kusmischew, Peters, Lubarsky, Kastalsky, Pallas); ex insula Unalaschka (Mertens); insulis oceani orientalis. St. Paul (herb. h. Petrop.); e. Dahuria (Treskin); Mandshuria ad fluv. Amur (Turcz.), ex Am. bor. - occid. (Richardson, Chamisso, Eschscholtz, Choris.) Lusum giganteum vidi e regione fluv. Mackenzie circa Fort Franklin (Richardson); Kamtschatka (Rieder.). - (Rch.)

Aster peregrinus Pursh. Fl. 2, p. 556.

Comparatis speciminibus numerosis authenticis cum pluribus aliis spontaneis cultisque, et siccis et vivis, persuasum mihi est, species sequentes nil nisi formas unius ejusdemque esse: A. salsuginosus Richards. in Frankl. Journ. to the Polar Sea App. p. 760. Torr. et Gr. Fl. of North-Am. 2, p. 155. A. unalaschkensis Less. in Linnaea. 6, p. 122. Bong. De veg. ins. Sitcha, p. 148. A. inuloides Fisch. herb. (non Don.) 
A. consanguineus Ledeb. Fl. ross. 2 , p. 473 et herb. (lusus parvus involucris caulibusque apice villosissimis). Variat:

ß. robustus. Glabrior, robustior; foliis radicalibus caulinisque inferioribus majoribus (cum petiolo saepe semipedalibus) late lanceolatospathulatis, superioribus ovato-lanceolatis (rarissime tantum ex eadem radice lineari-lanceolatis); floribus majoribus, involucri squamis pluribus angustioribus. - A. salsuginosus $\beta$. Torr. et Gr. 1 c, pro parte. Hook. in Bot. mag, t. 2942. Fl. bor. -am. 2, p. 6.

Vidi specimina e Kamtschatka (herb. Fisch.), Sitcha (Wrangell, Bongard, Eschscholtz), Kadjak (Tiling), ex Unalaschka (Chloris, Langsdorf, Kastalsky), insula Koränginsk (Mertens).

Var. $\beta$. ex Am. bor, in herb. Fisch. a cl. Hookero comm. et culta e seminibus olim a cl. Parry missis.

Obs. Specimina tantum utniflora, unico excepto, reperi. (Rch.)

\section{Closia chllensis ingl. ot xeke.}

Compositae Tubuliflorae Trib. III Asteroideae Subtrib. I Asterineae Div. II Astereae Subdiv. 6 Bellideae DC. Prodr. V.

Caule pulveraceo-puberulo pilis longis raris versus apicem chebrioribus intermixtis adsperso; foliis petiolatis pinnatilobatis lobis dentatis infimn bifido, inferjoribus petiolatis superioribus sessilibus. Herba annua? Caulis procumbens flexuosus, a basi valde ramosus, pilis brevissimis glanduliferis, longioribus raris eglandulosis intermixtis, puberulus. Folia pinnatilobata, lobis utrinque $2-3$ infima maxima plerumque biloba on.nibus grosse et acute dentatis, glabra vel pilis raris breviusculis conspersa, crispa, patentia, viridia, herbacea, inferiora cum petiolo $1 \frac{1}{2}$ pollicari $2^{3 / 4}$ pollices longa, $1 \frac{1}{2}$ pollices lata, superiora sensim minora. Rami infra receptaculum cavi. Capitula in ramis nudis vel microphyllis solitaria, heterogama, cum radio albo patentissimo $7 \frac{1}{2}$ lineas lata, disco $4-4 \frac{1}{2}$ lineas lato. Injolucrum semiglobosum, discum aequans, squamis biseriatis aequalibus, oblongis, acutis, plus minus obtuse carinatis, breviter sparseque glanduliferopuberulis, totidem herbaceịs, viridibus, numerosis, circiter 2 lineas longis. Receptaculum convexum demum planum, leviter foveolatum glabrum. Flores radiantes $11-14$ feminei, tubo angusto breviter glanduloso-puberul, lamina ovali vel spathulato-ovali obtuse tridentata alba. Flores discoider tubulosi, tubo a medio ad germen cylindrico angusto superne campanulato 4 dentato, dentibuis recurvatis, glandulifero-puberulo, flavo. Antherae 4 connatae, ecandatae, breviter et obtusiuscule sagittatae, apice appendiculato exsertae. Stylus cylindricus. Stigmata linearia longiuscula, revolita, omnia aequalia. Achaenium compressum, strigoso-pilosum, pilis acierum longioribus, obscure cinereum. Pappus perparvus, inconspicuus, uniserialis, paleis basi coalitis apice setaceo-multifidis connatus. - Closiae Cotulae Remy (Gay Fl. chilen!'4, 120 tab. 46 fig. 2) valde affinis 'sed nunquam in plantis nostris folia vidi bipinnatifida, qualia et decripsit et depinxit cl. Remy. Praeterea nescio, cur hic autor genus Closiam Ecclipteis annumeraverit, quum et receptaculum paleis careat et folia, paucis infimis exceptis, alternent et ad genus Brachycomen characteribus habituque proxime accedat. Differt tamen âb hoc genere receptaculo 
foveolato (nec alveolato), floribus disci 4-dentatis (nec 5-dentatis) squamis totidem herbaceis (nec margine submembranaceis). A genere Asteromaea Bl., cui propter pappum similis videtur, distinguitur habitu, stigmatibus linearibus, achaeniis plano-compressis ecostatis.

Patria: Chili, Paposo unde cl. Philippi mis. sub 1: 650. (Keke.)

\section{Cosmidium Purridgcanum stort.}

Glabrum, caule erecto striato; foliis oppositis v. alternis, pinnatisectis, foliolis pancis filiformibus subcarnosis; involucro exteriore sub 8-phyllo, phyllis appressis, lineari-subulatis, hispidis; involucro interiore exterius subduplo superante, phyllis ovato-oblongis, fuscis, margine albido scariosis, radii ligulis circiter 8 , cuneato-obovatis, apice grósse tricrenatis, atrofuscis, superne luteo-marginatis. Proxime accedit ad Coreopsidem tilifoliam Hook. Bot. Mag. tab. 3505 quae differt involucro exteriore patente, glabro, ligulis luteis, foliis pinnatifidis v. bipinnatifidis.

Patria Texas? (Rgl.)

Erigeron Karwingkyanus DC. Prodr. 5, p. 285.

Hujus synonyma sunt: Aster quercifolius Less. in coll. Schiedeana Schlchtd. in Linnaea 9, p. 265. (excl. squ.). E. heterophyllus Kth. et Bouché Ind. sem. h. Berol. 1845, p. 11. E. mucronatus DO. l. c.? Vittadinia triloba H. Paris, non DC. (Reh.)

Eupatorium Haageanum Rgl, et Kcke.

Eupatorium DC. prodr. 5, 141; ser. III Eximbricata; *) Capitulis $20-70$ floris.

Caule suffruticoso erecto cum petiolis breviter puberulo; foliis oppositis, petiolatis, ovatis, acuminatis, cordatis, subgrosse serratis, utrinque ad nervos levissime puberulis ceterum glabris, membranaceis; capitulis corymbosis pedicellatis circiter $35-40$ floris; bracteis involucri biseriatis, lineari-oblongis, acutis, puberulis; achaenio pentagono, hispidulo.

Caulis et rami teretes, laeves. Pili caulis et petioli curvato-arrecti, breviusculi, in corymbo paullo longiores et densigres; in nervis foliorum brevissimi et sparsi. Folia cum petiolo fere pollicari usque $3 \frac{3}{4}$ pollices longa, usque $2 \frac{1}{2}$ pollices lata, serraturis acutis. Corymbus tern alis, eapitulis $4=5 \frac{1}{2}$ lineas longis longiuscule pedicellatis circiter $50-100$ compositus, laxiusculus, inferne foliatus superne bracte is linearibus puberulis instructus; ramis alternantibus inferioribus arrecto-patentibus. Capitula campanulata circiter $4 \frac{1}{2}$ lineas longa. Flores albi. Pappus seaber, roseus, longus, persistens. Stigmata longe exerta. -

Haec species a cl. Haage sub nomine Eupatorii Fraseri 1086 missa differt ab hoc capitulis majoribus plurifloris. Foliis ad Eupatorium aromaticum L. et ageratoides $\mathrm{L}$. accedit sed facile dignoscitur capitulis majoribus laxius positis et achaeniis hispidulis. His vero accedit ad Eupatorium glechonophyllum Less., a quo differt corymbo multo uberiore, caule pilis curvatis puberulo (nec minutissime pulverulento- 


\section{$-4$}

puberulo), foliis majoribus acutis serratis, bracteis corymbi pro ratione brevioribus, pappo rosęo nee albo.

Patria ignota. (Kicke.)

\section{Eupatorlüu weinmannianum Rgl. et Kcke:}

\section{Eupatorium. Ser. III Eximbricata. ****) Capitulis 6-10 floris.}

Fruticosum, glabriusculum; ramis teretibus, laevibus, glabris; foliis oppositis, petiolatis, lato-lanceolatis, utrinque acutis vel acuminatis, serratis, basi apiceque integerrimis, glaberrimis, eglandulosis, penninerviis; corymbis terminalibus, amplis, laxiusculis, breviusculis, superne puberulis; capitulis $10-11$ floris; involucri cylindrici squamis linearibus, obtusis, ápice pubescentibus; achaenio quinquangulari, glabriuseulo. -

Folia herbacea basi, cuneata in petiolos subconnatos attenuata, dentibus majusculis acutis, nervo medio subtus prominente lateralibus vix prominulis, petiolo semitereti supra leviter canaliculato, cum petiolo $3-4 \frac{1}{2}$ pollices longa, $1^{1 / 2}-1^{3 / 4}$ pollices lata. Corymbi usque 9 pollices lati usque $4 \frac{1}{2}$ pollices longi, ranis oppositis, inferioribus elongatis et late patentibus vel fere patentissimis, bracteis lineari-subulatis instructi. Squamae subbiseriales, exteriores minores, basi bracteola una, vel duabus minoribus lineari-subulatis auctae. Receptaculum et florum fabrica ut in to to genere. Pappus albus raro apice subroseus.

Colitur in hortis sub nomine' Eupatorii glabri et Agerati glauei. (Kcke.)

\section{пужоdia alternifolla Rgl, et Kcke.}

Dysodia DC. prodr. V, 639, sect. II Boebera. Involucrum bracteatum, bracteis indivisis. Receptaculum brevissime fimbrilliferum aut subnudum. - Folia pinnatisecta.

Foliis infimis oppositis, reliquis alternis, pinnati-sectis, pinnis anguste Jinearibus, subtiliter mucronatis, integerrimis; pedunculo basi foliato superne bracteato, unifloro; involucro circiter 14-phyllo squamis concretis apice tantum liberis composito, bracteis $3-5$ la. ceolatis acuminatis glabris cincto, ligulis pluribus (circiter 15) patentissimis.

Planta $1 \% 2$ pede humilior. Caulis a basi ranosus; ramis ubique patentibus, glabriusculis linea una vel paucis a folio quoqne superiore decurrentibus notatis. Folia pleraque alterna sessilia, glabra, pinnatisecta, hinc inde glandula laete fusca conspersa, usque 10 lineas longa; pinnis utrinque 4 vel 5 , ultima minima, anguste linearibus, subtiliter mucronato-acutis, rhachi $1 / 3$-fere $1 / 2$ lineam lata angustionibus, longissimis fere 4 lineas longis. Pedunculn terminalis) glaber, erectus, monocephalus, $2^{\frac{1}{4}}-2^{3} / 4$ pollicaris, supra basim folio pinnatisecto instructus quod sequuntur bracteae plures distantes integerrimae lanceolatae acuminatae glabrae vel pilis brevissimis vix ciliolatae, glandulis 2 vel 4 rotwndis laete fuscis instructae arrectae, quorum infima interdum major linearis mucronato-acuta patula evadit. Involucrum turbinatum, glabrum, infra apicem glandulis oblongis laete fuscis conspersum, squanis ad apices acutinsculos tantum liberiș, bracteis is pedunculi similibus cinctum. Capitula heterogama, floribus radii femi- 
neis ligulatis, disci hermaphroditis tubulosis. Receptaculum convexum subalveolatum, vix fimbrilliferum. Ligulae patentissimae, oblongae, obtusae, apice levissime tridenticulatae, glabrae, pulchre flavae, 2 lineas longae. Discus ligulis subconcolor, $2 \frac{1}{2}$ lineas latus. Corolla florum discoideorum tubulosa, aequaliter 5-dentata, flava. Stamina ecaudata, apice appendiculata, inclusa, flava. Styli rami paullo exserti, recurvati, cono ad basim et apicem puberulo terminati. Achaenium elongatum, gracile, teretinsculum, griseum. Pappus uniserialis, paleis 10 basi integris apice in setas longas plures (rarius tres tantum) porrectas seauras divisis.

Patria ignota. Aecepta a cl. Haage sub nomine Hymenantheri tenuitolii. (Kcke.)

\section{Ginthera Exil.}

(Compositae. Subdivisio III. Heterotheceae. Endl: Gen. pag. 385 et suppl.)

Capitulum multiflorum, heterogamum; floribus radii subbiseriatis, ligulatis, foemineis; disci tubulosis, hermaphroditis. Involucri pauciseriati squamae imbricatae, lineares, basi adpressae, apice patulae, Receptaculum convexum, foveolatum; foveolarum marginibus dentatis. Corollae radii ligulatae; disci tubulosae, limbo quinquefido. Antherae in appendicem lanceolatam brevem excurrentes, basi muticae. Stigmata radii cylindrica, disci apice lanceolata hispida. Achaenia radii oblonga, laevia; disci cylindrica v. subcompressa, angulata, hispidula. Pappus radii nullus; disci coroniformis in aristas breves inaequales 1 vel plures excurrens.

\section{6. viscosa Rgl.}

Annua; caule erccto, superne paniculato-ramoso, 3-4 pedali, basi foliisque pubescente, superne foliis involucrisque plus minus viscosis; foliis sparsis, acutis, inferioribus oblongis, in petiolum attenuatis, plus minus dentatis; superioribus lanceolatis, v. lanceolato-linearibus subintegerrimis. Involucri squamae herbaceae, dorso impressopunctatae. Flores lutei; ligulae lineari-oblongae, obtusae, sub lente emarginatae. - Gutierrezia gymnospermoides hortul.

Patria: Texas v. America borealis. (Rgl.)

\section{Gactuca amurensis Mgl. et Max.}

Annua (?), glabra; caule erecto, tereti, folioso, apice paniculato; foliis subtus in costa media plus minus setosis, inferioribus in petiolum attenuatis, intermediis et superioribus basi sagittata sessilibus, oblongo-lanceolatis v. lineari-lanceolatis v. integris v. runcinato dentatis $v$. runcinato sinuato-pinnatifidis: lobis acuminatis $v$. longe acuminatis, integris v. praecipue basin versus sinuato-dentatis, v. rarius laciniatis, horizontaliter y. recurvo-patentibus; panicula elongata, contracta; capitulis pedicellatis, pedicellis bracteolatis; involucri foliolis obtusiusculis, violaceo-marginatis, inferioribus ovatis, superioribus oblongis; floribus flavis; achaeniis ellipticis, compressis, marginatis, 
utrinque uninerviis, tenuissime muriculatis, rostro discolori triplo longioribus. -

Planta $1-3$ pedalis. Folia supra viridia, subtus glauca, usque 6 pollices longa, varie incisa. Affinis L. elongatae Mühl. et altissimate Bieb. et strictae W. et K.. L. elongata panicula effusa, achaeniis lanceolatis costatis in rostrum breve concolor attenuatis, - L. altissima fol. subtus glabris, involucri squamis linearibus acuminatis nec marginatis, achaeniis anguste-lanceolatis obscure costatis, rostrum concolor subaequantibus, - et L. stricta panicula corymbosa, involncri squamis linearibus acuminatis nec marginatis, et achaeniis obverse-lanceolatis, subcostatis, rostrum concolor subaequantibus dignoscitur.

Ad fluvios Amur et Ussuri legit semina Maximowicz. (Rgl.)

Senecio nhllippieus Rgl, et Kcke.

Senecio L. sect II Radiati c, Plantae herbaceae *) Caules foliati et plerumque pluricephali. Remy in Gay Fl. chil. 4, pag. 194. Senecio ser. XII Chilenses. ****) Radiati, herbacei a, Hualtini, caule folioso, capituliz plurimis corymbosis. DC. prodr. VI, 417.

Ramis, foliis, involucro persistenter arachnoideo-tomentosis candicantibus; foliis in petiolum 'attenuatis, oblongis vel ovalibus, pinnatilobatis, superioribus lorato-pinnatipartitis; corymbo 6- vel 7-cephalo, inaequali, pedunculis distantibus; involucri campanulati squamis circiter 20, discum aequantibus; achaeniis puberulis, albido-cinereis.

Planta herbarea, circiter 19 pollicaris. Caulis a basi ramosus. Folia inferiora late oblonga, in petiolum alatum supra canaliculatum basi paullo ampliatum usque $11 / 2$ pollicarem attenuata, grosse dentata vel pinnatiloba, utrinque arachnoideo tomentosa, supra lana arachnoidea tenuiore et senectute paullo (non prorsus)detergibili vestita, subtus pilositate persistente nervis prominentibus, cum petiolo usque $3^{1}{ }_{4}$ pollices longa, usque $1 \frac{1}{4}$ pollices lata; superiora brevius petiolata basi semiamplexicauli subauriculata auriculis rotundatis, profundius et lyratopinnatilobis, lobis parce et minute dentatis; omnia herbacea. Rami foliosi, fragiles, pilositate foliorum. Corymbus terminalis, basi folio sessili amplexicauli auriculato pinnatilobo suffultus, laxus, subinaequalis, centrifuge-efflorescens, cum petiolo 13 pollicaris. Pedunculi bracte is linearibus integris (inferioribus semiamplexicaulibus auriculatis appressis) vestiti, infimus semipedalis, summus $1 \frac{1}{4}$ pollicaris. Capitula radiata, mediocria. Involucri squamae lineares, subulatae, apice fuscatae, basi calyculo appresso lineari-subulato circiter 11-phyllo cinctae. Receptaculum paleaceum, alveolatum. Ligulae 13, oblongae," apice obtuso retusae, glabrae, flavae, 6 , lineas longae, 2 lineas latae, Flores disci quinquedentati. Stylus exsertus. Achaenia teretia, pilis appressis brevibus puberula. Pappus scaber, caducus.

Chili, mis. Philippi sub 780 . (Keke.)

\section{Venlaium spectosum $\mathrm{Rgl}$. \\ it}

Annuum, acaule vel caulescens; caulibus erectis, monocephalis, ad apicem foliosis, sulcatis, foliis involucroque pilis articulátis patentibus albidis v. rubicundis dense molliterque vestitis; foliis basi exauricula- 
tis, uon decurrentibus: radicalibus et caulinis inferioribus petiolatis lyrato-pinnatifidis, lobis obtusis, lobo terminali maximo subrotundo $v$. ovalo sinuato-iobato crenatove, lobis lateralibus paucis repando-crenatis decurrentibus: caulinis superioribus decrescentibus, sessilibus, oblongo-lanceolatis, sinuato-dentatis; involucri squamis exterioribus lterbaceis, dense villosis, e basi lanceolata in apicem cochleariformem reflexum excurrentibus; interioribus appressis scariosis, ovato - oblongis, obtusissimis, glabris, luteolis; receptaculo alveolato, fimbrillifero; ligulis concoloribus, aurantiacis, apice minute tridentatis, pollicem et ultra longis, floribus disci tubulosis brevibus, limbo nigricante. Creseit in promontorio bonae spei.

V. calendulaceum, arctotoides et multiflorum Hort. - V. calendulaceun Less. foliis supra glabris subtus cano-tomentosis involucri squamis erectis. V. aretotoides Less. petiolis basi auriculatis caule foliisque albido-tomentosis involucri squamis erectis et V. subacaule D. C. cui proximum caule scapiformi apice nudo vix basi folioso dignoscitur. (Rgl.)

\section{Kopsia parallela Rgl, et Kcke.}

Foliorum nervis lateralibus numerosis parallelis vix arcuatis subsimplicibus ubique subaeque validis in nervum marginalem validum excurrentibus; lobis corollae prorsus albue ellipticis, acutiusculis; ligulis nectarii duabus; germine glabro.

Frutex, Ramorum cortex pallidus, sublaevis. Folia per 4 rarius per 3 verticillata, anguste lanceolata, longe et exacte acuminata, basi in petiolum brevem sensim sensimque attenuata, glabra, laete viridı, nervis lateratibus numerosis parallelis subsimplicibus et ubique subaeque validis in servum marginalem margini parallelum validum excurrentibus; cam petiolo usque 8 pollices longa, $1 \frac{1}{4}$ poll. lata. Inflorescentia terrsulitis subcymosi. Bracteae minimae acuminatae appressae glabrae viriles, Pedicelli circiter 4 lineas longi. Calyx quinquepartitus, glaber, eglandulosus, lobis lanceolatis acutis aequalibus valvatis parvis $3 / 4$ linean longis juventute imbricatis. Corolla hypocraterimorpha, tota alba, extus glabra; tubo quam calyx multoties longiore circiter pollicem longo augisto apice (ubi stamina insident) intlato, ad ostium faucis corona pilorum param intus flexa ornato, intus intra ostium glabro, ad altitudinem taminum pilis densis faucem claudentibus obtecto, infra stamina glive, limbi lobis ellipticis acutiusculis, tubo fere duplo brevioribus, $7 \mathrm{li}$ ne: tougis, $2^{3} \frac{1}{4}$ lineas latis. Staminas in parte inflata tubi inserta; antheris luccolatis acuminatis filamento longioribus. Nectarium duplex et liguis $2 \mathrm{um}$ germinibus alternantibus glabris constructun. Germina 2 Glubra, facie incerna plana appressa. Gemmulae circiter $12-16$ a medio af basiu placentae in ovarium prominentis nascentes. Stylus 1 tubum coroliae subaequans. Stigma subito incrassatum, apice leviter bilobum ct be isborm appressorum corona pilorum exili ornatum, basi annwo superne piturtoso in medio coarctato cinctum. - Tubus corollae, fisma et pilositate et insertione staminum optime congruit cum ic. Hooker, in Bot. Mag, tab. 4220 Kopsiae fruticosae, si coronam pifortu in ostio summo faucis neglexeris. Ne sectio transversaria quidem (fig. 4)germinis minus quadrat. Sed, si corollam Kopsiae fruticosae DC. et arboreas BI. cun cl. Candolleo in aestivatione sinistrorsum con- 
tortam appellaveris, tum in nostra specie eam dextrorsum contortam esse dices. Praeterea numeris gemmularum valde recedit a charactere et Candolleano et Blumeano hujus generis. Sed propter nectarium insignem notasque aliasque hane speciem nervatione folionum prae reliquis valde peculiarem (a qua nomen triviale desunptum) generi Konsiae subjungendam esse censeo. (Kicke.)

\section{Dracocephaium argunense Fisch.}

Species optima ab aff. D. Ruyschiano facillime dignoscitur calycibus apertis conspicue infundibuliformibus, dentibus tribus superioribus subaequilatis; corollis duplo longioribus subtriplo latioribus subito intlatis et praecipue antherarum loculis, in D. Ruyschiano medio solum barbatis, tota longitudine villoso-ciliatis. - Specimina numerosa in hortis e seminibus enata spontaneis constanter conformia nulloque modo variabilia inveni.

D. organense Fisch. Hort. Gorenk, et herb. Rehb. Magaz. p. 1, t. 1. Lk. Enum. h. Berol. 2, p. 118. D. Ruyschiana $\beta$. speciosum Ledeb. Fl. ross. 3 , p. 390 et herb. Turcz. Fl, baic. - dah. v. 2, fase. 1, p. 412 (p. 411 post lin. 12 ponendum)..- (Rch.)

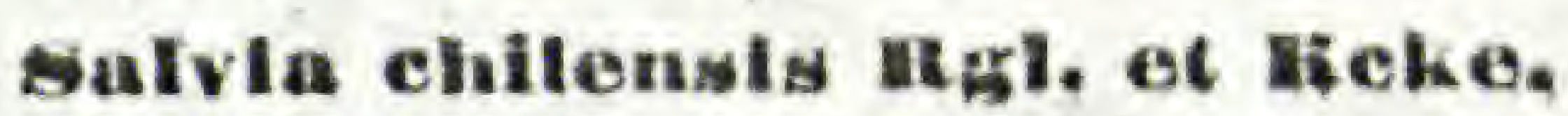

Salvia sect. VII Calosphace $\$ 6$ Longiflorae, L. Tubiflorae Bentham in DC. prodr. 12,338 ,

Herbacea; ramis pubescentibus; foliis petiolatis, ovatis, obtusis, profunde cordatis, crenatis, supra brevissime puberulis nitidis subtus incano-pubescentibus herbaceis; racemis et calycibus glanduloso-pubescentibus, viscosis; verticillastris bifloris, remotis; calycis tubulosi labio superiore integro, cum dentibus duobus labii inferioris acuto et recto; corolla calycem plus duplo superante, coccinea, hirsuta, labio superiore concavo truncato levissime emarginato, inferiore breviore trilobo.

Rami pilis brevibus deflexis densis hinc inde pilo longiore intermixto pubescentes. Folia qupra leviter rugulosa saturate viridia et propter pilos brevis imos tactu mollia, subtus pallide viridia et pilis brevibus pratesertim in nervis dense molliterque pubescentia; petiolo $1-1^{1 / 4}$ pollices longo ad modum ramorum pubescente, lamina $1^{1}{ }_{2}-$ $2^{1 / 2}$ pollices longa $14-24$ lineas lata. Racemi usque 5 pollicares vel
demum longiores, ut calyx pilis patentissimis viscido-glaubilosis pubescentes. Bracteae late lanceolato-ovatae, acuminatae, brevissime puberulae, virides, deciduae, Pedicelli pilis brevibus densis deflexis pubescentes, usque $2 \frac{1}{2}$ lineas longi. Calyx 12 -nervoso-costatus, viridis, circiter 6 lineas longus. Corolla usque 14 lineas longa, parum incurva, intus annulo piloso carente; labii inferioris lobi obtusi laterales minores subarrecti, medius latior lato-obovatus basi subito in unguiculum brevissimum angustatus apice rotundato integerrimus vel emarginatus et in emarginatura apiculo inflexo ins actus. Stamina 2 paullo exserta; filamentis.brevibus; conneçtiris longissimis purpureis glahris, hinc erectis et apice antherae loculum dorso tilacino-purphream gerentibus, illine deflexis et basi clavato-dilatata connatis. Stylus glaber 
apice bifido exsertus, lobis acutiusculis altero majore.-Videtur affinis Salviae squalenti H.B. Kth. sed differt foliis herbaceis (nec coriaceis), caule ramisque non piloso-glutinosis, corolla coccinea nec purpurea, noti aliis. - A Salvia Gilliesii Benth. usque adhuc unica in terra chilensi inventa differt caule herbaceo (nec fruticoso), ramis nec canotomentosis nec glandulis coccineis conspersis, foliis neque truncatocordatis neque subtus tomentosis, calycibus pedunculatis neque subsessilibus; corolla coccinea neque azurea, stylo exserto glabro neque brevissimo pubescente, odore nullo.

Chili: Paposo leg. et semina comm. cl.Philippi sub 16 775.(Kcke).

\section{Casselia diversifolia $\mathrm{Kgl}$.}

Huc pertinet C. serrata N. ab E. et C. integrifolia N. ab. E. (Nova acta ac. Caes. XI pag. 75). Folia plantae nostrae v. integerrima v. plus minus grosse serrata. (Rgl.)

upomoea odoratissima Jacq. Hort. Schönbr. II tab. 229, $\beta$. Intermedia rigl.

Foliis $2-4$ jugis; foliolis anguste lineari-lanceolatis v. sublanceolatis, usque $1 \frac{1}{3}$ poll. longis. (Rgl.)

\section{Ipomoea charwinskiana Rgl.}

Caule volubili, foliis calycibusque glabris; foliis cordatis, acuminatis, integerrimis; petiolo basi subtorto quam folium paullo breviore; cymis dichotomis plurifloris, pedunculo communi quam folium breviore; sepalis ovato-oblongis, ex apice obtusissimo mucronatis; usque $3 / 4$ poll. longis; exterioribus brevioribus, atroviridibus, crassis, costato-rugulosis: interioribus pallidioribus tenuioribus medio costatis; floribus magnis, speciosis, albis, basi intus purpureis. -

Radix crassa subtuberosa. Caulis inferne sublignosus. Folia usque $2^{1 / 2}$ poll. longa et $1^{3 / 4}$ poll. lata. Corolla circiter $2^{1 / 2}$ poll. longa. (Rg1.)

Aftinis J. verrucosae Blume. Semina misit Karwinsky e Mexico

Gilia lutea steud. $\beta$. aurea agl.

Leptosiphon aureum Hort. Floribus aureis.'(Rgl.)

\section{Calceolaria slutinosa veer. et $\mathrm{kgl}$.}

Ind. sem, horti Turic. 1848.

\section{Var. californiea.}

c. californica Hort.

Canle robusto erecto, foliis superioribus pinnatis; pinnulis ovatolanceolatis v. ovatis, pinnatifidis v. duplicato inciso-dentatis, basi stipitatis v. rarius confluentibus. (Rgl.) 
Caule tetragono, calyceque sparsim viscoso-puberulo: -

A. salicariaefolia, Gardneri et minor dignoscitur :

A. salicariaefolia Humb.' et Bonpl.; annua, labio inferiore ceteris lobis corollae longiore.

A. minor Fisch. et Mey.; humilio, suffruticosa, flore minore, labio . inferiore ceteros lobos corollas aequante. Variat:

$\alpha$. glabra; glaberrima, caule tereti.

ß. puberula; vid. supra.

A. Gardneri IJook. suffruticosa, labio inferiore ceteris lobis corollae longiore. (Rgl.)

Adhatoda Neen ab. Es. 'Tyloglossa D. C. Prodr. XI, pag. 389.

Calyx profunde quinquefidus, laciniis aequalibus vel subaequalibus. Corolla ringens, tubo brevi mediocri v. elongato, labio superiore concavo erecto apice bidentato, inferiore plano reflexo trilobo, palato inflato utrinque lineis gibbis transversis notato. Stamina 2, tubo infra medium inserta et ad faucem connata. Antherae biloculares, loculis connectivo obliquo subrhomboideo impositis, altero altius inserto, inferiore basi calcari incurvo superiore calcari simili sed, brevissimo vel plerumque nullo. Stigma parvum obtusum. Germen superum conoideum, glandula crassa annulari cinctum, biloculare, loculis biovulatis.

Herbae v. frutices foliis oppositis integris. Flores v. axillares solitarii glomerative v. apicem versus in spicam plus minus foliosam conferti, v. spicae axillares, v. spica spicaeve terminales v. in panicula spiciformi terminali congestị. Bracteae magnae. Bracteolae oppositae.

\section{Adhotbda aurantiaca ngl.}

Kastera aurantiaca Rgl. Grtfl. 57.

Cyrtanthera aurantiaca Hook. Bot Mag, tab. 4468,

Frutex $4-5$ pedalis, ramis elongatis, flexuosis, nodosis, lineis 4 decurrentibus subquadrangulis, puberulis et deinde scabriusculis. Folia ovato-oblonga, subacuta, integerrima v. repanda, ciliolata, supra obscure viridia nitida, subtus pallidiora et in nervis scabriuscula, in petiolum brevem attenuata, $3-3^{1 / 2}$ poll. longa et $1^{1 / 2}$ poll. lata. Panicula spiciformis, conferta vel basi interrupta, bracteis calycibus floribusque puberulis; paniculae ramis abbreviatis, plurifloris. Bracteae penninerviae, acutae, 'inferiores foliaceae ellipticae, superiores oblongo-ellipticae, subcoloratae Bracteolae calycem aequantes, ut calycis lobi lineari-lanceolatae. Flores longe exserti, pulchre aurantiaci, usque 2 poll. longi; tubo gracili costato basi inflato; lobis labii inferioris oblongis subaequalibus. Stamina corolla paullo breviora. India occidentalis.

Proxime accedit ad Adhatodam 3 Tyloglossa Sect. E. N. ab. E. in DC. Prodr. XI pag. 406, 'differt antem corollae tubo elongato palato inflato, calycis laciniis subaequalibus et inflorescentia. Cyrtanthera antheris brevibus, basi muticis, loculis antrorsum contiguis, connectivo apice mucronato, palato non inflato vix penninervio dignoscitur. (Rgl.) 


\section{Körnickea mgl.}

Calycis tubus eum basi ovarii connatus, 5-partitus, laciniis 2 inferioribus ceteris paullo longioribus. Corolla perigyna ventricoso-infundibuliformis, sursum curvata, in calyce nutans, tuho basi contracto aequali, limbo ringente erecto-patente 5-lobo. Stamina inclusa, corollae basi inserta, quinto sterili, antheris ovatis liberis. Annulus perigynus, pentagonus, integer, tenuis. Ovarium apice liberum. Stigma bilobum.

Herba propagulis squamoso-amentaceis perennis. Caules humiles, erecti, foliosi, foliis apice confertis, floribus axillaribus. Habitus Niphaeae, flores Scheeriae.

\section{Körnickea lanata IRgl.}

Mandirola lanata Pl. et Lind. in Lind. Cat. et Lem. in Ill. Hort. tab. 80. Scheeria lanata Hanst. in Linnaea XXVII, 715 et 757.

Caulis $2-3$ pollices altus, foliisque dense albo-lanatus. Folia opposita, petiolata, ovata, obtusiuscula, crenata, supra minus lanata. Pedicelli axillares, solitarii, uniflori, folium superantes, cum calycibus villosi. Calycis laciniae lanceolato-oblongae. Flores pallide lilacini, violaceo picti. - Mexico. (Rgl.)

\section{Dodocatheon integrifolium Mlelax.}

Plantae genuinae specimina numerosa e seminibus a cl. Tilingio in insula Kadjak lectis enata variant foliis integerrimis denticulatis repandisve, sed filamentorum tubum connatum semper exsertum inveni. (Reh.)

\section{Erica Rinzi I Rgl.}

E. intermedia Hort.

Hiabitu et colore viridi ab E. Bovieana diversa. Planta hybrida inter E. Bovieanam Lodd. et gilvam Wendl. (Rgl.)

\section{Echinocactus fossulatus Pfeifr. et otto Abbild. II, tab. 13. Var. quadrispinus.}

Cinereo-viridis; costis semper 8 , latis; aculeis plerumque 4 , oppositis, rarius $5-6$, centrali nullo; petalis albis extus stria rubra notatis. -

Karwinsky misit ex Las Ranchos de Medina. (Rgl.)

\section{Saxifraga stelleriana Merck.}

Species optima primo intuitu foliis viridibus lineari-spathulatis calycisque segmentis obtusis rarissime tantum breviter mucronulatis ab al ibus distincta. - Minime S. nitida Schreb., cujus icon in Sternbg. liev. t. 10, b. f. 4 et ej. descriptio I. c. Suppl. p. 94 optime convenit cum $\mathrm{S}$. tenellae speciminibus Wulffenianis in herb. Mertensiano. 
S. Stelleriana Merck. in herb. Pall. (e specim. Pall. in herb. Fisch.). Ser, in DC. Prodr. 4, p. 46, Ledeb. Fl. ross. 2, p. 208. S. nitida Ledeb. 1. c. p. 207 (nec Schreb. nee. DC.). S. nitida $\beta$. glabra. Rgl. et Til. Fl. ajan. n. 121. S. Kruhseana Ser. 1: c. herb. Fisch. S. pseudoburseriana herb. Fisch. Cham. in Linfaca 6, p. 555 (sub s. spec. nov.) et in herb. Ledeb. Torr, et Gir. Fl. of North Am. 1, p. 565.

Vidi specimina e Sibiria orientali (Pallas), pr. Ischiga (Krulıse); terra Tschuktschorum ad sinum St. Laurentii (Eschscholtz, Choris); Kamlschatka (Eschscholtz, Postels, Lubarsky, Merck, Kastalsky, Kúsmisczew, Langsdorf, Stubę̧dorf); ex insula Koränginsk (Postels); Ajan ('Tiling.). - (Rich.)

\section{Gaxifraga unibrosa th. var. crenulata ugl, et, Rach.}

(S. umbrosa $\beta$. hybrida H. Petrop.)

Certo non hybrida, sed var. pulchella foliis vix angustioribus elegantissime minute crepulatis pilis copiosioribus adpersis insignis. (Reh.)

\section{Saxifraga eunelfolla K. petlolls elliatlo.}

(S. hirsuta $\beta$. Huworthii H. Petrop.)

Non differt nisi petiolis plerumque usque ad apicem pilis longis ciliatis, qui in genuina nunc desint nunc basi tantum occurrunt. (Rch.)

\section{Escállonia rubra Pers.}

i.

Haec planta mirum in modum variat et glandulis et pilis et magnitudine florum foliorumque neque non horum forma. Has variationes quamquam a cl. Endlicher et Poeppig in Nov, Gen. et'Sp. pl. 1, 9 optime jam expositas, tamen autores recentiores neglexerunt. Folia enim variant oblongo-spathulata vel obovata vel ovato-lanceolata, adulta 9 lineas $-3^{1 / 4}$ pollices longa atque 3 lineas - fere $13 / 4$ pollices lata. Eadem subtus punctis resinosis plus minus elevatis crebris parcisve vel gaudent vel carent. Rami nunc pubescent nunc glabrescent, nunc cum calyce glandulis pedicellatis obsiti sunt, une iis carent. Pedunculi nunc uniflori nunc pluriflori, flores nunc rubri nunc albi evadunt. Hanc ob causam cl. Poeppig et Endlicher tres varietates constituerunt :

A. Rubra uniflora: Pedunculis simplicibus unifloris; calycibus glabris; floribus rubris. (E. rubra Hook. Bot. Niag. t. 2890).

B. Rubra multiflora: Pedunculis ramosis, multifloris, ealycibus glanduloso-pubescentibus; floribus rubris.

Poepp. et Endl. Nov. Gen. et Sp. pl. 1, tab, 15. Stereoxylon rubrum R. et P. Fl. per. 315 , tab. 236, fig. 6.

C. E. rubra albiflora. Pedunculis simplicibus ramosisve, uni-multifloris; calycibus glanduloso-pubescentibus; floribus albis.

E. Poeppigiana DC. prodr. 4, 3.

Suppetunt mihi specimina permulta et culta et spontanea, quibus examinatis has varietates illas A e $B$ paullo aliter intelligo. Etenim 
pedunculi uniflor in speciminibus uberioribus facile pluriflori ovadunt. Formae igitur variae tali modo mihi sese offerunt:

\section{A. Rubriftora}

a. micrantha; floribus angustioribus.

a. vera: pedunculis calycibusque glandulosis.

Stereoxylon rubrum R. et P. Fl, per. 3, 15, tab. 236 , fig. 6 .

Eseallonia rubra Pers. ench. 2, 255. DC. prodr. 4, 3. Poepp. et Endl. Nov. Gen. et Sp.pl. 1, tab. 15. Lodd. Cab. tab. 1659.

Escallonia rubra Pers. var. B. multiflora Poepp: et Enld. Noy. Gen. et Sp. pl. 1, 9.

ß. glabrescens: pedunculis calycibusque glabris.

Escallonía rubra, Hook. Bot. Mag. tab 2890. Griseb.

in Philippi pl. chil. exsicc. 132 (transitus ad var. $a$ ).

Escallonia rubra Pers. var. A. uniflora Poepp. et Endl. Nov, gen. et sp. pl, 1, 9.

b. macrantha: floribus latioribus.

Escallonia macrantha Hook, et Arn. in Hook. Bot. Misc.

3, 341 (v. s.). Hook. Bot. Mag. 4473. V. Houtte Fl. d. Serr.

6 , tab. 632 et Lem. Jardin Fleur. 1, tab. 55 (Icon utraque e Hook. Bot. Mag. iterata!). Morren Belg. Hort. 3, tab. 1, tig. 7.

Escallonia punctata Miq. in Lechler pl. chil. exsicc. Af 586 vix DC.

Folia hujus varietatis, quae cl. Hooker obtusiuscula appellat, in specimine authentico exactissime acuta sunt. Praeter flores latiores discrimen ab E. rubra Pers. vera non inveni.

\section{B. Albiflora}

a. glandulosa: pedunculis calycibusque glandulosis.

Escallonia Poeppigiana DC. prodr. IV, 3 (anno 1830). Remy in Gay Fl. chil. 3, 51 .

Escallonia glandulosa Lodd. Bot. Cab. 1291 (anno 1827).

Sweet Brit. Fl. Gard. ser. 2, tom. 1, tab. 81 (anno 1831).

Escallonia rubra Pers. var. C. albiflora Poepp. et Endl. Nov. Gen. et Sp. pl. 1, 6.

b. glabrescens: pedunculis calycibusque glabris.

Escallonia Grahamiana Gill. in Hook. Bot. Misc. 3, 343 (anno 1833). E. rubricaulis Colla in Mem. di Torino t. 37, p. 79 (Opus non vidi). Remy in Gay Fl. chil. 3, 57 .

Haec varietas, cum puncta resinosa in latere inferiore demum prorsus desint, in Escalloniam rubricaulem Coll. incidere videtur. Pedunculi plantae nostrae etiam longi evadunt. (Kcke.)

Eseallonia hinda Lk, et otto. Ic. 1, tab. 23. (anno 1828).

E. montevidensis DC. prodr. 4, 4 (anno 1830).

E. floribunda $\beta$. montevidensis Cham. et Schl, in Linnaea $1826,543$.

Var, $\alpha$, genuina: floribus albis.

Var. $\beta$. rosea: floribus roseis. 


\section{:- $51-$}

Escallonia Organensis Gardn, in Hook. Ic. pl. new. ser. 2, tab. 514. Ilook. Bot. Mag. tab, 4274.

$V$ ar. $\beta$ a varietate $\alpha$ non diflert nisi florum colore (et c-lyce puberulo?). Species et foliis et panicula brevi congesta multiflora insignis. Puncta resinosa in pagina folii inferiore ut in speciebus aliis numc adsunt nunc desunt. (Kcke:)

Baucus torlloldes nc. Pr. IV, pag. 214.

Huc pertinent D. australis Clos in Gay IIstoria phys. et pol. de Chile tom III, pag. 136. D. montawus W. in herb, Berlandiero differt involucelli phyllis pedicellur subaequantibus. In planta nostra involucelli phylla pedicellis multo breviora, simplicia v. laciniata. Semina misit Dr. Philippi e Chili. (Rgl.)

Daucus montevidensis nc. prodr. 4, 214.

D. hispidifolius Clos in Gay Fl. chilen. 3, 135.

Planta nostra a cl. Philippi e Chili sub 664 missa optime congruit cam planta Cumingiana chilena. Speciminia a cl. Otto e horto Berolinensi missa paullo differunt canle inferne hispidiore et lobulis foliorum parum angustioribus. Diagnosis Closiana D. hispidifolii prorsus in nostram plantam saepe unbella plus quam 10-radiata praeditam cadit. (Keke.)

\section{Aquilegla parvinora nedeb.}

In Ledeb. Ic. fl. alt, t.' 108 hujus sepala in viva planta reflexa errore erecta (sine dubio ad siccam) delineatia sunt. (Reh.)

Trollius europacuis h. $\beta$. humilis DC. Prodr. 1, p. 45.

Trollius calthaeoides Schrad. e seminibus ex h. Götting. acceptis enatus, hujus varietatis lusus pedunculatus est. Specimen T. calthaeoidis herb. Schrad. ad 'T. europaei var. $\gamma$. DC. l. c. spectat. (Rich.)

\section{Lepidlum áurieulatum ngl. et lneké.}

Lepidium R. Br. sect. VI Dilepticum DC. prodr. I, 205.

Anuuum; caule decumbente ramoso; foliis glabris sessilibus auriculatis pinnatifissis, pinnulis utrinque 2 vel 3 linearibus acutis integerrimis lamina folii angustioribus; floribus inconspicuis parvis viridibus; petalis nullis; staminibus 2; silicula suborbiculari apice breviter emarginata.

Annumm. Caulis a basi decumbens et ramosus, cum ramis pedicellisque pilis brevissimis patentissimis pubescens. Folia glabriuscula sessilia, pinnatifissa, pinnis utrinque 2 vel 3 in summis una acutis integerrimis patentibus'; folia caulis et rami cujusque duo vel tria infima angustiora exauriculata pipuis laminam latitudine subaequantibus reli- 
qua latiora pinnis lamina angustioribus auriculata auriculis acutis; fere usque $1 \frac{1}{2}$ pollices longa. Racemi elongati usque 5-pollicares. Flores parvi virides. Calyx 4-partitus, deciduus. Sepala oblonga, acuta, navicularia, dorso puberula vel glaberrima, viridia. Sepala nulla. Stamina 2 (cum valvis alternantia) sepalis vix longiora. Pedicelli fructiferi patentissimi longitudiue siliculae. Silicula suborbiculata, leviter et obtuse emarginata, glabra; valvulis carinatis apice obtusis. Stigma sessile. (Kcke.)

\section{Psychlne stylosa Desf. $\beta$. longibracteata Egl.}

Bracteis siliculas superantibus v. aequantibus; floribus siliculisque subsessilibus; silicula hastato-obcordata, apice late emarginata.

Plantam genuinam descripsit cl. Desfontaine (Fl. atl. II, pag, 69, tab. 148) bracteis silicula brevioribus, superioribus longitudine pedicelli, floribus siliculisque pedicellatis, silicula apice trumcata. An planta nostra species distincta? ( $\mathrm{Rgl}$.)

stenopetalum sphaerocarpnm F. Muill. (Plantae Müllerianae exsiccatae.)

Annuum, erectum, ramosum, glabrescens, in partibus junioribus pilis minutis glanduliferis munitum, ramis patentibus; foliis radicalibus inferioribusque caulinis pinnati-partitis, laciniis utrinque $1-3$ linearibus obtusiusculis, supremis linearibus integerrimis v. utrinque dente acuto; racemis fructiferis elongatis, laxis, subsecundis; sepalis oblongis, obtusis, pedicellum deinde deflexum aequantibus; petalis calyee plus duplo longioribus, lineari-spathulatis, ungniculatis, apice in processum subulatum croceum exeuntibus; siliculis subglobosis, stigmate apiculatis, bilocularibus, utrinque $3-7$-spermis. - Port Lincoln in Nova Hollandia meridionali. Semina Ferd. Müller misit (Rg1.)

Passinora coriacea Juss. in Ann. du Mus. VI, tab. 39.

Var. marmorata.

P. marmorea v. Houtte Cat.

Foliis divaricato-bilobis (lobo intermedio nullo), lobis acuminatis, supra ad nervos argenteis. Cetera ut genuinae. (Rgl.)

\section{Cerastiun perfoliatum $\mathbf{A}$.}

Var. a. glaberrimum; fol. margine glabris. (C. chloraefolium Fisch.) Var. $\beta$. ciliatum; fol. margine ciliatis. (C. perfoliatum F. et M.) (Rgl.)

\section{Modiola caroliniana Don.}

(M. caroliniana $\beta$. Cumingiana C. A. Mey.)

Hace varietas, in Ind. 11 sem. h. Petrop. Suppl. p. 58 a cl. Meyero descripta, speciei genuinate merus lusus est, sed inconstantissimus, 
nam in uno eodemque ramo folia plus mints profunde incisa profert, ut jam e figura bona Dillenii H. elth. t. 4. f. 4. elucet. Nostra, c seminilues plantae authenticae enața, nunc omnino forman vulgarem M. carolinianae exhibet. (Rcli.)

Pachiza stenopetala Casaretto Nov. stirp. Bras. Dec. 21?

Foliolis $7-11$ articulatis, sessilibus, cuneato-vèl lanceolato-oblongis, acutis vel acuminatis, longe mucronatis, versus basim sensim attenuatis, glabris; pedunculo longitudine calycis, cum eo levissime vel vix puberulo et glandulis fusco-aureis parvis nbique consperso; calyce tereti, truncato, minute quinquedentato; sepalis ligulatis, velutinis; tubo filamentorum quam calyx quadruplo longiore, cum filamentis albido; antheris oblongis, rectiusculis.

Inermis. Rami glabri, virides. Folia alterna, petiolata, petiolo $13 \frac{4}{4}-5$ pollicari tereti glabro viridi. Foliola digitata, supra obscure viridia et nitida, subtus pärum pallidiora et opaca, nervo medio praesertim subtus promínente, 2-fere 6 pollices longa, $41 / 2-15$ lineas lata. Flores axillares. Pedunculus longitudine calycis, $3 / 4$ pollicaris. Calyx tubulosus viridis, basi corona glandularum majorum destitutus, intus sericeus, fere $3 / 4$ pollicem longus, fere 5 lineas latus. Petala 5 , lineari-ligulata, obtusiuscula et apice cucullata, utrinque breviter velutina, viridia, $6^{1} / 2$ pollices longa, infra medium 5 infra apicem 6 lineas lata. Staminum numerosissimorum filamenta a basi usque ad apicem $5 \frac{1}{2}-6$ pollicaria, alba, inferne in tubum glabrum ant infra medium vel ubique pilis stellatis albido-velutinum $2 \frac{1}{2}-3^{1 / 2}$ pollices longum 3 lineas in diametro latum connata, supra tubum in fasciculos polyadelphos usque $\beta / 4$ pollicarès soluta, superne bina fere usque ad apicem concreta versus apicem libera. Antherae oblongae, rectae, flavae, supra basim affixae. Germen superum, ovatum, quinqueloculare, placentis parietalibus usque in medium porrigentibus ibique connatis et quasi centralibus; loculis pluriovulatis. ovulis in angulo centrali affixis. Stylus inferne molliter pubescens vel villosus, superne glaber, longitudine staminum. Stigma leviter quinquelobum. - Foliolis numerosis apice acutis vel acuminatis et mucrone longo mstructis insignis. Quum cl. Gasaretto in descriptione Pachirae stenopetalae apices foliolorum prae ceteris insignes prorsus neglexerit, certo statuere non potui, num planta nostra in speciom illam incidat. Praeterea descriptio Casarettiana differt calyce sinuato (non truncato et quinquedenticulato), petalis angustioribus, sed forsan propter exsiccationem speciminum. (Keke.)

\section{Citrus juponica Thbrg: n. Jap. pag. 29z. Icon. n. jap. tab. 15.}

Caule fruticoso humili, ramis angulatis; petiolis anguste alatis; foliis ovato-oblongis, acutiusculis, crenulato-serrulatis; floribus axillaribus solitariis v. geminis 20 - 25 -andris; fruetibus ovatis apice umbonatis, pulpa acri. - C. Bigeradia Poitean et Risso. Var. chinensis Bosse Handb. I, pag. 552. C. sinensis Risso. - Flores, albi extus purpurascentes. - Japan. Chlua. (Rgl.) 
Malpighla coddigesli aggl.

(D. C. Prodr. I, pag. 577. 1. Urentes.)

Ramis decumbenti-hispidis; foliis lineari-lanceolatis, sinuato-subdentatis, denticulis muticis v. seta pungente terminatis; floribus erectopatentibus. M. aquifolia Lodd. bot. cab. tab. 1079.

Frutex $3-5$ pedalis, ramosus; ramis confertis, subhorizontaliter patentibus, pilis dichotomis decumbentıbus dense strigosis. Folia disticha, brevissime petiolata, e basi rotundata v. subcordata lineari-lanceolata, denticulis muticis v. seta pungenti caduca terminatis; supra atroviridia, nitentia, glabra; subtus pallidiora, pilis dichotomis decumbent ${ }^{3}$ us adspersa; $1^{1} / 2$ pollices longa, $1 / 8$ poll. lata. Pedunculi erectopatentes, uniflori, solitarii v. bni, folio breviores, medio articulati, vix pilosuli. Calyx basi glandulis 8 instructus. Petala unguiculata, rosea, calycem multo superantia, lamina subrotunda, undulata, plus minus fimbriata. Patria: India occidentalis.

$\mathrm{Ab}$ affini M. aquifolia L. (D. C. Prodr. I, pag. 568. Plum, et Burm. tab. 168 fig. 1.) nostra species dignoscitur: foliis undulatis, sinuatosubdentatis, dentibus muticis v. spinoso-setosis, floribus erectis, (in M. aquifolia secundum tab. cit. Plumierii flores penduli sunt) et ramis strigoso-hispidis. (Rgl.)

Maytenus miodelianus n. Petrop. Index sem. 4. Petrop. 56, pag. 36.

Variat. calycibus bracteolisque glanduloso-ciliatis v. glabris, petalis acutis v, obtusiusculis.

ß. oblongifolius; foliis lanceolato-oblongis, obtuse acuminatis, calycibus bracteolisque evidentius glanduloso-ciliatis. (Rgl.)

Eupliorhia odontophylla willd. Suppl. horti bot. Berol. pag. 28.

Iruticosa; seminuda; $7-12$ angularis, angulis dentatis, dentibus junioribus apice foliosis; aculeis solitariis $\mathrm{v}$. teruis, bracteolis munitis, apice floriferis.

Caulis carnosus, e basi rarius apicem versum ramosus, 7 - 12 angularis, dentatus; dentibus minutis, junioribus apice foliolo minuto lanceolato carinato acuto terminatis. Aculei recti, fusci, $1 / 3$ poll. longi, bracteolis 3-pluribus muniti, in dentium sinubus inserti, juniores apice floriferi; bracteolis junioribus ovatis, obtusis, pubescentibus, rubicundis, deinde fuscis angustioribus. Flores masculi parvi, in apice aculeorum solitarii. Involucelli foliola exteriora in hracteolas transeuntia, ovata, obtusa, extus puberula, rubra; foliola interiora 5, glanduliformia, horizontaliter patentia, lenticularia, atropurpurea, impressopunctata. Stamina infra medium articulata, squamis subulatis ciliatis basi fuita. ( $\mathrm{Kgl}$.)

Adenandra untnora BartI. Wendl. Beitr. I, 78. D. C. Prodr. I, pag. 713 sub Jiosma uniffora. 


\section{$-\quad 55$}

var. villosa Rgl.

Ramulis hirsutis; foliis calycibusque hirsuto-ciliatis. - A formosa Hort. (Rgl.)

\section{oxalis rhombeo-ovata sit. Wil. 11. bras.}

Walp. Rep. I, pag. 489.

Huc pertinet O. Barrelieri Hook. Bot. Mag. tab. 3784. (Rgl.)

\section{Gxalis clandestina Philippi Linn. 28,616.}

Oxalis L. \$5. Flores rosei vel violacei, in apice pedunculi conmunis umbellati. Folia composita e tribus vel raro e quatuor foliolis obcordatis vel emarginatis. M. Barneond in Gay FI. chilena 1, pag. 450:- $-\$ 5$. Acaules aut stipite nudo apice tantun folioso donatae, pedunculis uni-bi-ant saepius multifloris, foliis radicalibus petiolatis plurifoliatis saepius trifoliatis. DC. prodr. 1, 695 .

Annua, petiolis, pedunculis (et communi et partialibus), calyce, capsula patentissimo et glanduloso pilosis. Caule brevissimo, densissime folioso. Foliis ternatis, foliolis late obovatis, profunde emarginatis, utrinque sparse pilosis; pedunculo communi cum partialibus per 2-4 umbellatis simplicibus folia circiter aequante; sepalis concoloribus; corolla parva quam calyx paullo longiore; petalis spathulatis, erectis, obtusissimis, integerrinis vel leviter retusis, saturate roseis, fauce viridi.

Planta vix 3 pollices alta. Petiolus basi supra planus et membranaceo-alatus, 2 - ultra $21 / 2$ pollicaris. Foliola sessilia, plana, praesertim subtus impresso-punctulata, herbacea, supra saturate-subtus pallide viridia, fere usque 6 dineas longa et lata. Pedunculns communis supra basim articulatus, ubique teres, $2 \frac{1}{4}$ pollicaris. Umbella basi bracteis 2 vel raro pluribus linearibus glabris albidis vel rubellis suffulta. Pedunculi partiales erecti vel parum nutantes, breves, usque $4 \mathrm{l} / 2$ lineares vel breviores. Flos perparvus, sine pedicello 2 lineas longus. Sepala oblongd, acutascula, laete viridia, erecta, in capsula submatura apice patentia. Petala spathulata, obtusissima, integerrima vel leviter retusa, versus basim connata, vix 2 lineas longa, 1 linea angustiora. Capsula calycem aequans, 5-angulata, 5-locularis, loculicide dehiscens septis post dehiscentiam etiam connatis, pilis patentibus glanduliferis obsessa. Senina fusca, rugulosa. (Kicke.) ?

Detomeris maerodon Naud. in Ainn. d. sc. nat., 3 ser. IV, 52, tab. 2, fig. III C.

Planta nostra a descriptione Naudiniana differt ranis supremis petiolis junioribus paniculisque dense albo - (nec fertugineo -) villosis; calyce reflexo - (nec arrecto-patenti) villoso. (Kcke.) 
Myrtus pulehella ingl.

(Leucomyrtus, Horibus 5-fidis.)

Frutex humilis, cortice brunneo ramulis virgatis petiolis pedicellis calycibusque pubescentibus; foliis oppositis, coriaceis, brevi-petiolatis oblongis et apicem versus attenuatis, mucronato-acutis, integerrimis. supra deinde glabrescentibus, subtus dense sericeo-pilosis, $1-1 \frac{1}{3}$ poll. longis et usque $1 / 3$ poll. latis; floribus axillaribus; pedicellis apice bibracteatis, solitariis, unifloris, folio duplo brevioribus; petalis albis ruseo rinctis, margiue pilosis. -

Folia trinervia, nervis lateralibus juxta marginem positis. Flores iis M. communi duplo minores. Bracteolae lineari-subulatae rubicundae, calycem subaequantes. Calycis lobi ovati, breviter acuteque acuminati, glanduloso-punctati.

M. myricoidi H. B, M. proxime affinis et in hortis sub nomine M. tenuifoliae cuita. Illa pedicellis folia subaequantibus, foliis latioribus subaveniis supra glabris et petalis glabris, - hatec foliis linearibus (unciam longis, lineam latis), calycibus glabris dignoscitur. (Rgl.)

\section{Melaleuca affinis ingl. et kieke.}

Glabra; ramis laxis; foliis remotiusculis, oppositis, oblongis, obtusis, uninerviis senectute trinerviis, planis, viridibus; spicis cylindricis, densifloris, pallide lilacinis; hypanthio adnato; phalangiis polyndris, unguibus nullis.

Arbor parva uitra 5 pedalis, glabra. Rami virgati, laxi, glabrj. Folia opposita, subsessilia, oblonga, obtusa, juvenilia margine levissime puberula cito glaberrima, uninervia, senectute trinervia et rubella, punctata, plana, coriacea, arrecta vel patentia, viridia, 5 lineas longa, 1 lineam lata. Spicae in medio ramo, raro in axi nondum innovata terminales, densiflorae, cylindricae, pallide lilacinae senectute sordide albidae, $1 / 2-3 / 4$ pollicem longae vel paullo breviores, rhachi glabra. Hypanthium adnatum, breviter cylindricum, glabrum, punctatum. Sepala glabra, brevia, latissime ovata, rotundato-obtusa, glabra, rubromarginata. Petala illis multo majora, semiorbicularia, basi truncata, exnnguiculata, glabra, pallide lilacina. Phalangia brevissima, polyandra. Filamenta pallide lilacina, petalis multo longiora. Stylus filiformis, inclusus, petalis brevior. Stigma punctatum. - Diu dubius haesitavi, num planta Mülleriana varietatem tantum Melaleucae decussatae R. Br. an speciem distinctam exhibeat, quum forma foliorum spicarumque atque fabrica florum illi speciei valde affinis sit. Habitu vero adeo discrepat, ut mihi speciem peculiarem constituere videatur. Rami enim laxi et virgati (nec rigidi et virgati), folia remota viridia (nec conferta nec intense glauca) vix decussata pro ratione longitudinis panllo angustiora plantae nostrae faciem prorsus aliam reddunt. Ad hoc accedit, quod folia uninervia senectute demum (tunc rubella) trinervia appareant atque petala unguibus careant, quae in M. decussata unguibus brevibus quidem sed distinctis gaudent. Ad Melalencam decussatum tamen nisi me specimen cultum Herb. Fischeriani fallit, M. tetragona Lodd. pertinere videtur.

Patria: Nova Hollandia unde mis. Ferd. Muller sub of 3790. (Kcke.) 


\section{j

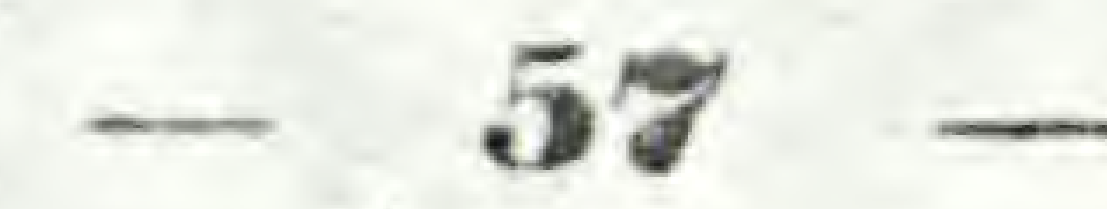 \\ Genm nutana Eam. \\ (6. affine II. Petrop.)}

G. affine lusus inconstans est pilorum caulinorum Jongiorum defectu vix distinctus. (Reh.)

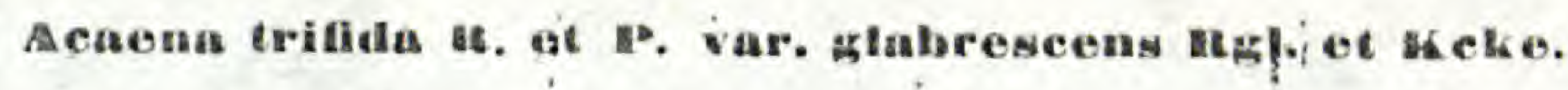

Prater foliorum rhachin sparse pilosam glabra.

Specimina hujus speciei spontanea e Chili allata vario modo pilosa vel villosa evadunt, praterea vero cum nostra planta congruant. (Kicke.)

Spiraça confusa Rgl. et Kcke,

Sp. chamaedrifolia Aut. nec Linné

et

Spiraea ehamaedrifolla L. nec Aut. plur.

Spiraea confusa in hortis nostris minime rara plurisque timen antoribus nomine fals $\rho$ Spiraeate chamaedrifoliae audit. Quam enim plurimi Spiraeam ulmifoliam Scop. appellant, nulla est nisi Spiraeat chamaedrifolia L. vera; quatn vero iidem nomine Spiraeae chamaedrifo. liae vocant, hanc nomine Spiraea confusae nunc salutamus quun e neque nomen autorum illud debeat neque alind sit impositum.

Cl. Linné ipse Sp. chámadrifoliam vix vidit, sed quum diagnosin suam e descriptione Ammani "Spiraeae Chamaedryos foliis" sumat eum tanquam autorem laudat. Cl. Ammanus autem (Stirp. var. in imp. ruth. sp. proven, ic. et deseript. pag. 190 A 269) plantae illi prater alia "ramos angulosos" et ,folia in ambitu argute dentata" adscribit. Haec prorsus in eam plantam cadunt, quam cl. Scopoli Spiraeam ulmifoliam appellavit; quae vero plerisque autoribus Spiraea chanaedrifolia est, haec ramis teretibts nec angulatis atque oliis aut vix aut versus apicem tantim serratis gaudet, basi semper integerrima. Praeterea autem utraque species cl. Turczaninowio in Fl. Baical. 1378 et 379 (sub Sp. Hexuosa Fisch. et Spiraea chamaedrifolia) monente facillime distinguitur carpellis.

Sequitur igitur compáratio diagnostica utriusque speciei.

\section{splraea cenfusa Rgl, et Kcke.}

Sp. chamacdrifolia C. Koch in Rgl. Gartfl., 1855, pag. 403. Turcz. Fl. Baic. 1, M 379.

Ramis teretibus, exangulatis; foliis vel integerrimis vel apice vel ab apice ad medium serratis; carpellis apice intus gibbis stylo demum patentissimo et quasi infra apicem posito.

Foliorum majorum ambitus subovalis vel ellipticus. 
Pall. F1. russ. 1, tab. 15. (V. sp). florifer.! Fructus in icone inaccurate depicti in descriptione bene delineati.) Ledeb. Fl. ross. 2,14 !

sp. ulmifolia Scop. 2, 349, tab. 22. Koch Syuops. 1. 245. C. Koch in Rgl. Garthl. 1855, 402 .

sp. Hexuos Fisch.! in DC. prodr, 2, 542. Cambess, mont. Spir. in ann. d. sc. nat. 1, 365. 1. 26. C. Koch in Rgl. Liartil. $1854,403$.

Ramis angulatís; foliis vel integerrimis vel apice vel toto ambitu serratis aut inciso-serratis; carpellis extus gibbis, stylo suberecto manifeste apicali.

Folia plerumque magis et acutius et profundius serrata quam in snecie pracedente, atque si majora plerumque ovata (maxima latituline

i approximatit. Spiraeam flexuosam prorsus eandem esse specimina Herbari Fiseheriau docent, quod sub nomine sp. flexuosae var. latifoliae formum vulgaren grandifoliam et sub nomine Sp. flexuosae var. angustifoliae formam foliis minoribus instructin a cl. Cambessertes depictam conservavit. (Kcke.)

\section{Spiraea mexicana H. Petrop.}

S. ariaefoliae Sm. proxime affinis. Dignoscitur foliis minoribns ramulis angulatis, floribus racemosis $\mathbf{v}$. paniculatis, paniculae ramis sparsis. reflexis. Patria ignota. (Rgl.)

\section{Luplnus nootkatensin Donn. Cat. Cant.}

(L. Blaschkeanus Fisch. et Mey.)

Indumentum caulium interdum nsque a! basin pilis adpressis obsitorum ex una eademque radice enatorum maxine variat; carina in spreininibus cultis spontaneisque nunc villoso-ciliata, nune basi vix ciliolata vel glabra Legumina pubescentia omnino similia sunt. L. Blaschkeamum (C. A. Mey. in Ind. 11, sem. h. Petrop. Suppl. p. 57 et in herb. Fisch.) igitur nil nisi L. nootkatensis lusum esse canlibus superue adpresse pubescentibus carinisque villoso-ciliatis convictus sian.

Teste cl, Kusmisczew, cujus specimina unalaschkana in herb. Fischeri asservantur, hujus speciei radix longissima flava erudit et cocta sapida est. (Rch.)

\section{oxylobinm capliatum Benth. $\beta$. Angustifolium Rol.}

Foliis oppositis, lineari-lanceolatis, mucronato-icutis v. rarius oblongo-lanceolatis, ex apice rotundato-mucronatis. (Rgl.)

\section{Pultenacas Ottonis mal.}

(Pultenaea Sect. 1. Hymenota D. C. Pr. 11. p. 110.)

Alipesse pubescens; fol, anguste-linearibus, subacerosis, obusis, 
apicem versus paullo latioribus, subtus convexis, supra canaliculatis ; stipulis subulatis, parvis, membranaceis; floribus terminalibus, solitariis; bracteis ovatis, scariosis, inbricatis, calycis basin cingentibus; hracteolis lanceolatis, horizontaliter patentibus.

Frutex humilis, ramulis sparsis. Folia obseure-viridia. Calyx sericeo-pilosus. Corolla aurantiaca. - Latrobea Brunonis H. Hamburg. Pultenaea Isrunonis, Benth. Aun. in Wien. Mus. I1, 81, cui planta nostra maxime affinis facile dignoscitur: stipulis uullis, bracteis nullis vel minimis. - Patria: Nova Hollandia. (Rgl.)

Templetonia retusa $\mathrm{k}$. Ar. $\beta$. angustifotia.

Templ. angustifolia II. Petrop. Foliis oblongo-linearibus. (Rgl.)

Dr. Liber thare a Euenter, horti praefectus.
Dr. s. megel, horti director.

Dr. Wretraiche, custos.

a. Hach, custos.

Petropoli, Decembri 1857. 